San Jose State University

SJSU ScholarWorks

Master's Theses

Master's Theses and Graduate Research

2002

\title{
Geophysical studies of the Lavic Lake Fault, Lavic Lake Play A, California
}

Halle R. Morrison

San Jose State University

Follow this and additional works at: https://scholarworks.sjsu.edu/etd_theses

\section{Recommended Citation}

Morrison, Halle R., "Geophysical studies of the Lavic Lake Fault, Lavic Lake Play A, California" (2002).

Master's Theses. 2327.

DOI: https://doi.org/10.31979/etd.jmks-f6ev

https://scholarworks.sjsu.edu/etd_theses/2327

This Thesis is brought to you for free and open access by the Master's Theses and Graduate Research at SJSU ScholarWorks. It has been accepted for inclusion in Master's Theses by an authorized administrator of SJSU ScholarWorks. For more information, please contact scholarworks@sjsu.edu. 


\section{INFORMATION TO USERS}

This manuscript has been reproduced from the microfilm master. UMI films the text directly from the original or copy submitted. Thus, some thesis and dissertation copies are in typewriter face, while others may be from any type of computer printer.

The quality of this reproduction is dependent upon the quality of the copy submitted. Broken or indistinct print, colored or poor quality illustrations and photographs, print bleedthrough, substandard margins, and improper alignment can adversely affect reproduction.

In the unlikely event that the author did not send UMI a complete manuscript and there are missing pages, these will be noted. Also, if unauthorized copyright material had to be removed, a note will indicate the deletion.

Oversize materials (e.g., maps, drawings, charts) are reproduced by sectioning the original, beginning at the upper left-hand corner and continuing from left to right in equal sections with small overlaps.

ProQuest Information and Learning 300 North Zeeb Road, Ann Arbor, MI 48106-1346 USA 800-521-0600

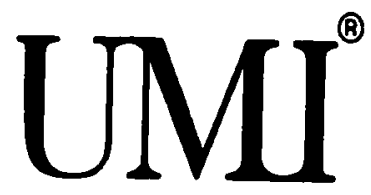




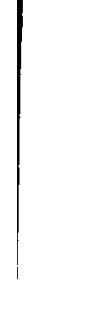

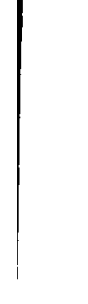

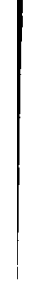

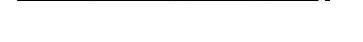

(1) 


\title{
NOTE TO USERS
}

\section{This reproduction is the best copy available.}

\author{
UMI
}



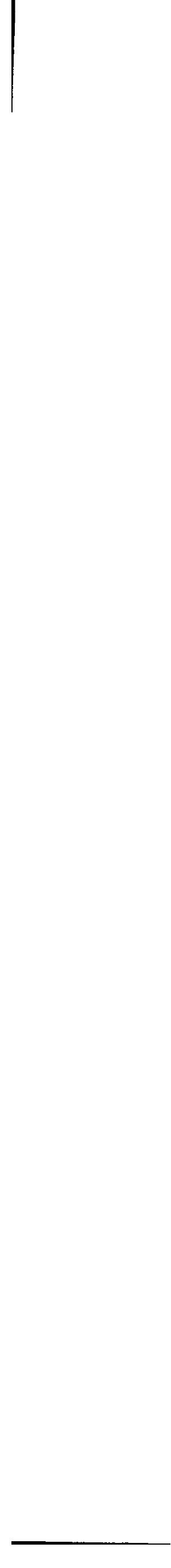

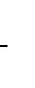
tra

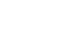

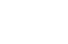

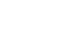

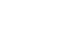

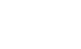

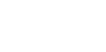

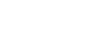

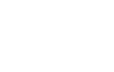

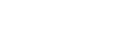

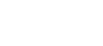

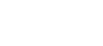

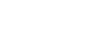

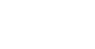

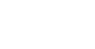

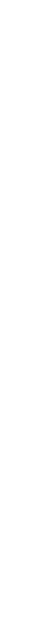




\title{
GEOPHYSICAL STUDIES OF THE LAVIC LAKE FAULT,
} LAVIC LAKE PLAYA, CALIFORNIA

\author{
A Thesis \\ Presented to \\ The Faculty of the Department of Geology \\ San José State University \\ In Partial Fulfillment \\ Of the Requirements for the Degree \\ Master of Science
}

By

Halle R. Morrison

August 2002 
UMI Number: 1410427

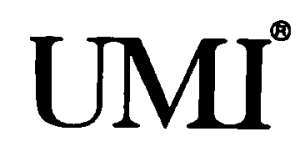

UMI Microform 1410427

Copyright 2002 by ProQuest Information and Learning Company. All rights reserved. This microform edition is protected against unauthorized copying under Title 17, United States Code.

ProQuest Information and Learning Company

300 North Zeeb Road

P.O. Box 1346

Ann Arbor, MI 48106-1346 
C 2002

Halle R. Morrison

\section{ALL RIGHTS RESERVED}


APPROVED FOR THE DEPARTMENT OF GEOLOGY

Dr. Richard Sedlock

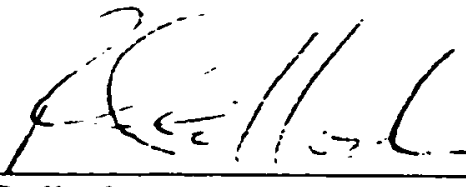

i aula Mescina

Dr. Paula Messina

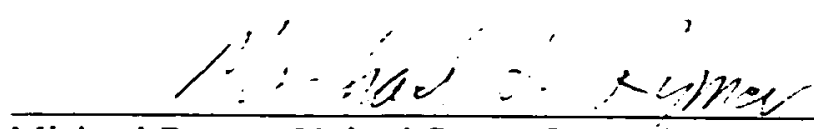

Michael Rymer, United States Geological Survey

APPROVED FOR THE UNIVERSITY

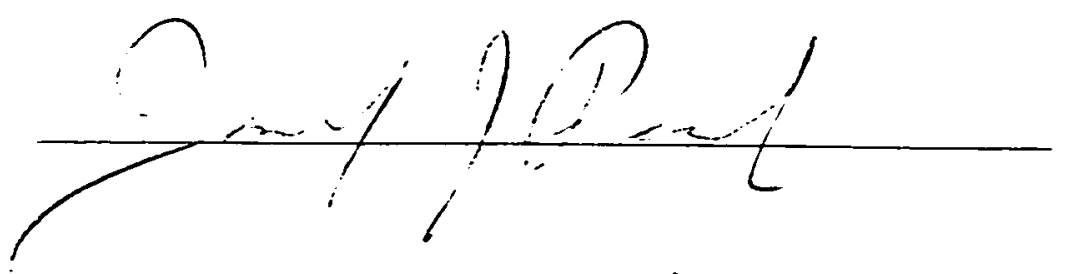




\title{
ABSTRACT \\ GEOPHYSICAL STUDIES OF THE LAVIC LAKE FAULT, LAVIC LAKE PLAYA, CALIFORNIA
}

\author{
by Halle R. Morrison
}

This study uses geophysical techniques to evaluate evidence of previous movement on the Lavic Lake fault, Mojave Desert, southern Califormia. Of primary interest in this thesis is the southwest section of Lavic Lake playa. The preferred interpretation of geophysical data is that basalt buried beneath part of the playa has an irregular topography, inconsistent with an undisturbed lava flow. Geophysical data confirmed the presence of unusual buried features in close proximity to the Lavic Lake fault. Seismic refraction revealed the possibility that buried or absent basalt somehow influenced the morphology of the 1999 Hector Mine earthquake rupture. Most basalt is found at considerable depths. Observations lead to several kinematic models. The fissure flow model uses the depth of the basalt to suggest that the Lavic Lake fault ivas once host to a fissure flow that erupted the basalt found along the Lavic Lake fault. Faulting has since altered surficial topography. 


\section{Acknowledgments}

This research was made possible through the support of a State Lottery Funds grant award through San José State University (SJSU), the Bertha Kalm Scholarship at SJSU, a Departmental Thesis award through SJSU, and a grant through the United States Geological Survey (USGS) Earthquake Hazards Team. Many thanks go out to Richard Sedlock of SJSU for advising this project, reviewing the manuscript, assistance with field work, helping out with the process, and just being an all-around great guy. Many thanks also to Paula Messina of SJSU and Michael Rymer of the USGS for their advice and enthusiasm during this project. I would like to acknowledge the generosity of the Marine Corps at Twentynine Palms Air-Ground Combat Center for access to their training grounds on Lavic Lake, and W. Karl Gross of the USGS for arranging access to the base. General assistance was also graciously provided by John Tinsley, Lucy Jones, and Mary Lou Zoback of the USGS, Paul and Stephanie Clark of Salinas, California, and Chris Madden of Central Washington University. Finally, a million thank yous go out to Richard ONeil of California State University. Northridge for his generosity and exceptional job as my field assistant. His work has been invaluable to the completion of this project. 
Table of Contents

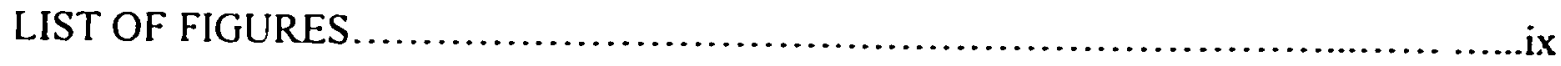

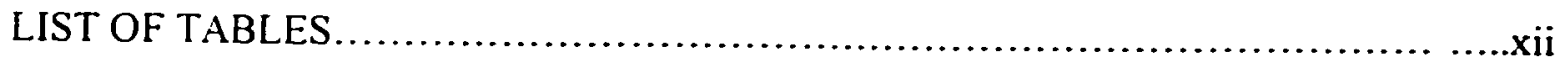

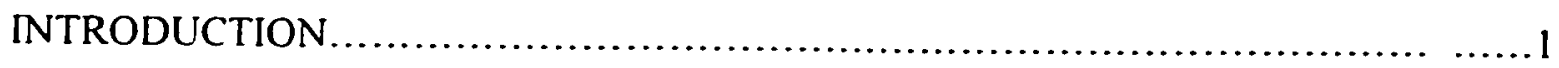

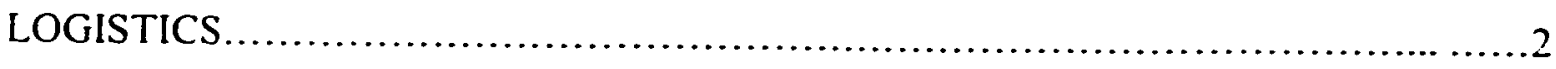

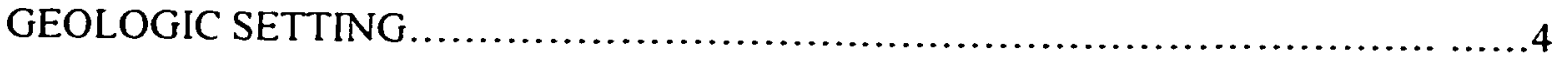

The Mojave Block and the Eastern California Shear Zone.........................4

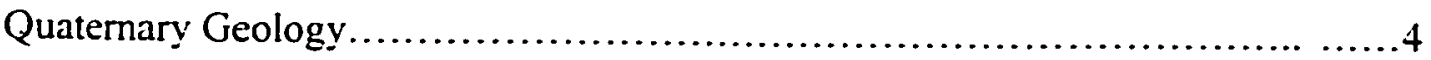

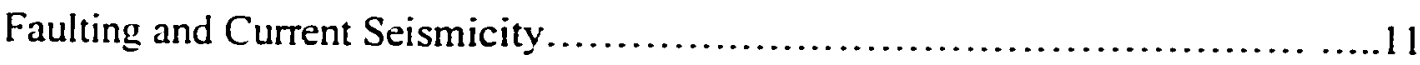

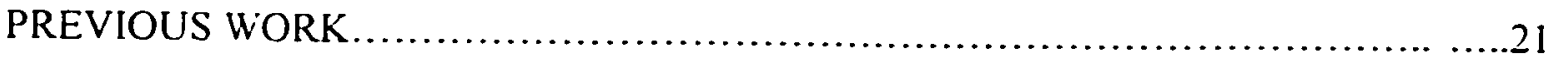

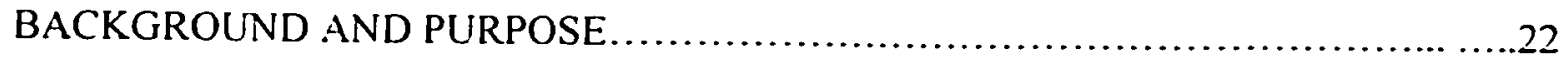

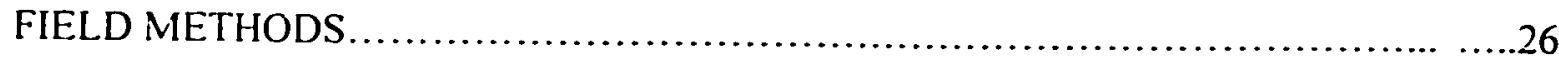

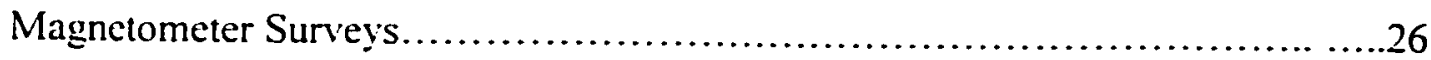

Seismic Retraction Surveys...........................................26

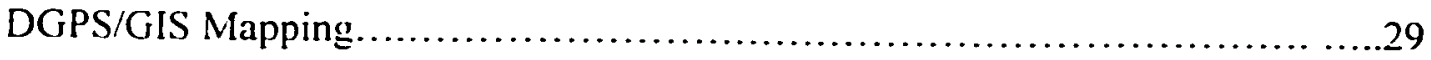

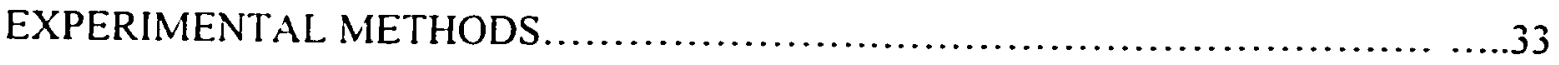

Magnetic Data....................................................... 33

Seismic Refraction Data..............................................

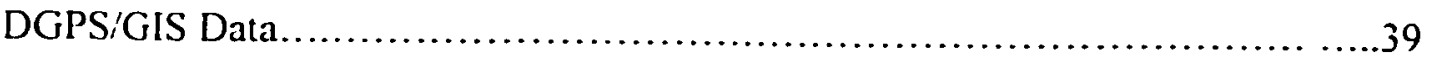

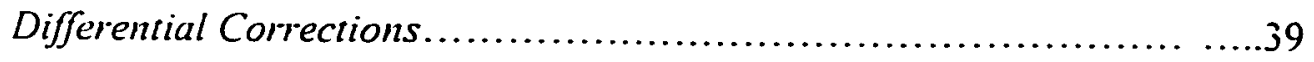

ArcView 3.2a Data Manipulation........................................39 
Magnetic Anomalies and Modeling.

Magnetic Anomaly. Contour Mapping.

Trends in the Linear Magnetic Profiles Across the Hand.

Trends in the Linear Magnetic Profiles in Playa Sediment.

Trends in the Magnetic Profiles Across the Hector Mine Rupture...... .....52

Trends in Other Linear Magnetic Profiles.............................56

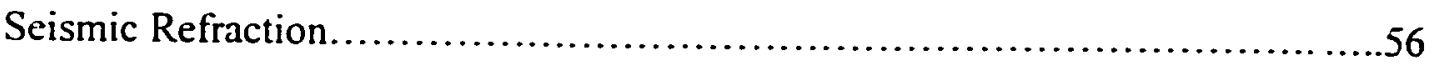

Seismic Lines West of the Hand .....................................56

Seismic Lines East of the Hand .....................................66

Seismic Lines South of the Hand ......................................68

Seismic Lines Across the Hector Mine Rupture.........................70

Seismic Lines Across a Major Stepover in the Rupture....................70

DGPS/GIS Data..................................................

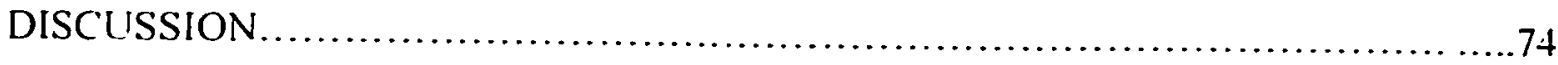

Depth of the Basalt-Sediment Contact in the Subsurface........................74

Geometry of the Basalt-Sediment Contact in the Subsurface.....................75

The Basalt-Sediment Contact in Relation to the Hector Mine Rupture.............76

Geomorphic Features of the Sunshine Peak Flow and Lavic Lake Playa...........77 


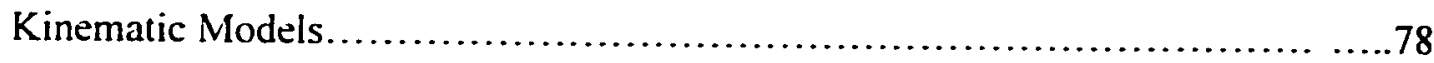

The Fissure Flow Model ...........................................78

The Multiple Dip-Slip Thrusting Model...............................80

The Basalt Exhumation Model......................................83

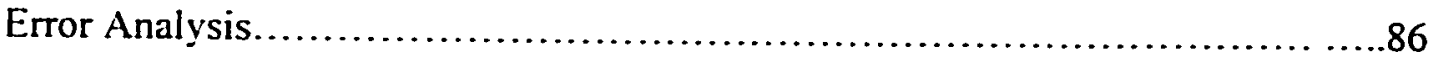

Magnetic Surveys.................................................86

Seismic Refraction Surveys.......................................87

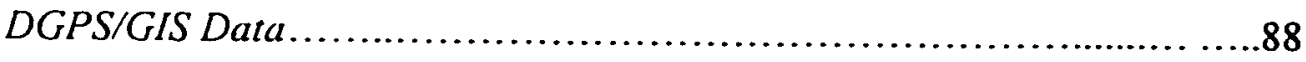

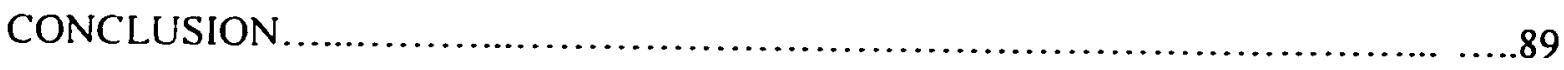

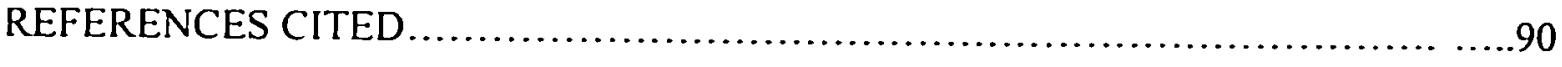

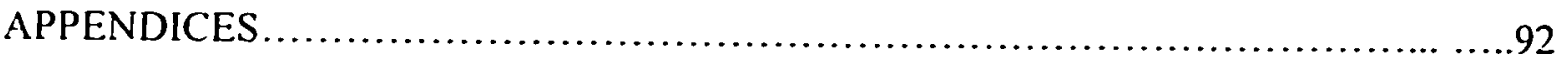




\section{List of Figures}

Figure 1. Generalized map showing active faults of the southeastern Mojave tectonic block. Map modified from Rymer et al. (2002).

Figure 2. Generalized geologic map of the Lavic Lake area. Map from Dibblee (1966).

Figure 3. Photograph of surficial basalt units found on the northern tip of the hand in Lavic Lake playa.

Figure 4. Photograph of surficial basalt units found within the hand near the Hector Mine earthquake rupture. Prominent fracturing is evident.

Figure 5. Photograph of in place surficial basalt units. Prominent fracturing is evident.

Figure 6. Photograph of basalt in the hand, close to the elevation of the sand and clay units of Lavic Lake playa.

Figure 7. Photograph of surficial clay and sand units that compose most of Lavic Lake playa. Photo was taken facing to the north.

Figure 8. Photograph of surficial clay units, with minor, shallow polygonal desiccation cracks and open fissure. The fissure formed from movement on the Lavic Lake fault.

Figure 9. Vertical section of playa exposed due to the Hector Mine earthquake.

Figure 10. Section of rupture between the hand and frisbee. Photograph was taken looking towards the south. Compression due to right-lateral movement is shown by playa sediment sheets being thrust towards the observer.

Figure 11. Section of rupture cutting through the frisbee. Photograph was taken looking towards the northwest. Vertical relief is due to minor east-vergent thrusting.

Figure 12. Section of rupture south of the hand. Photograph was taken looking to the south.

Figure 13. Major strike-slip faults in the area of Lavic Lake, with the principal rupture of the Hector Mine earthquake shown in yellow. Map from USGS, SCEC, and CDMG (2000) 
Figure 14. Photograph of portion of the Lavic Lake fault rupture and the Pisgah fault offset, about $2 \mathrm{~km}$ to the west. This photograph was taken looking towards the ivest.

Figure 15. Portion of 1:10,000 scale aerial photograph of study area in Lavic Lake, indicating the trace of the 1999 rupture

Figure 16. Locations of all magnetometer lines along which data was collected. This figure was generated by rover files imported into ArcView 3.2a.

Figure 17. Locations of all seismic refraction lines along which data was collected. This figure was generated by rover files imported into ArcView 3.2a

Figures 18 and 19. Photographs of field apparatus for seismic refraction data collection. These seismic lines extend across the Hector Mine rupture, near the north end of the finger

Figure 20. Map of the field area showing the rough spatial relations of the hand, the frisbee, the Hector Mine rupture, and associated units. 31

Figure 21. Plot of diurnal variation in the magnetic field at $34.66 \mathrm{~N}, 116.36 \mathrm{~W}$ $(0.50 \mathrm{~km}$ in elevation) according to the IGRF2000 model.

Figure 22. Portion of $1: 250,000$ scale aeromagnetic anomaly map of San Bernardino county. Map modified from Department of Conservation, Mines, and Geology ( I 988)

Figure 23. Magnetic anomaly contour map of the field area..............................1

Figure 24. Magnetic anomaly plot of magnetometer line $1 \ldots \ldots \ldots \ldots \ldots \ldots \ldots \ldots \ldots+3$

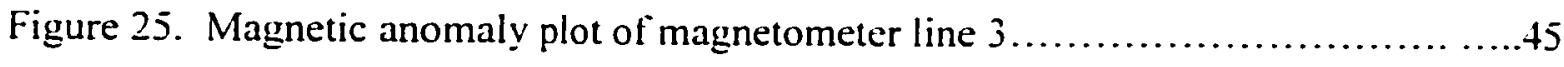

Figure 26. Magnetic anomaly plot of magnetometer line $4 \ldots \ldots \ldots \ldots \ldots \ldots \ldots \ldots \ldots \ldots$

Figure 27. Magnetic anomaly plot of magnetometer line $29 \ldots \ldots \ldots \ldots \ldots \ldots \ldots \ldots \ldots . \ldots \ldots$

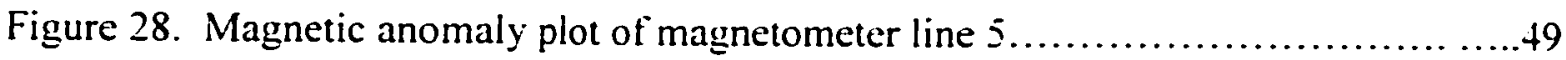

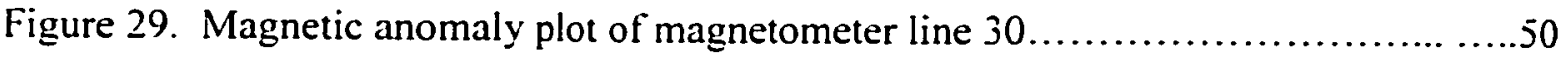

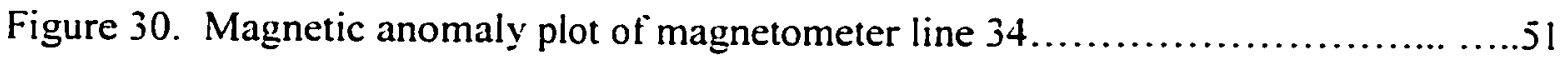

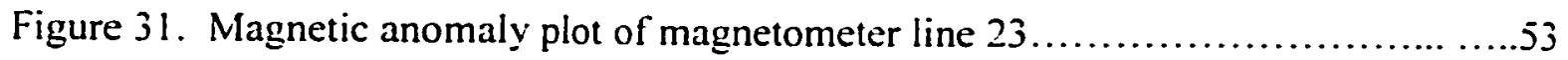




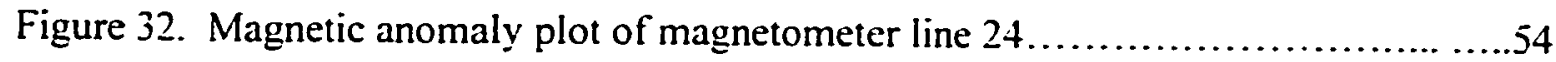

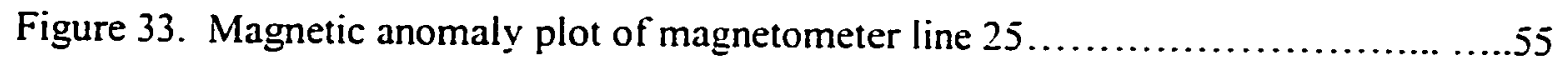

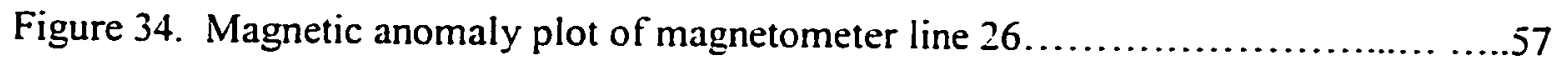

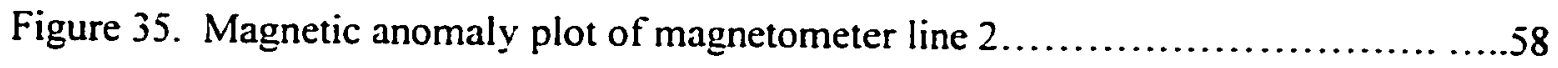

Figure 36. Three-dimensional DEM of the field area, showing the extent of major units. This figure was generated by rover files imported into ArcView $3.2 \mathrm{a} . \ldots \ldots \ldots . . . .72$

Figure 37. Three-dimensional DEM of the field area, showing the extent of major units, minimum, and calculated depths to basalt bedrock. This figure was generated

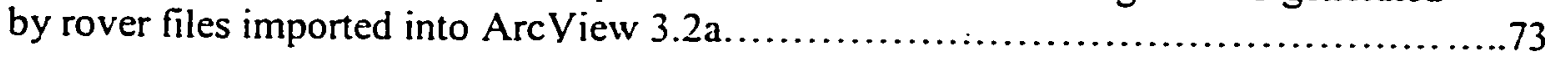

Figure 38. Times 1, 2, and 3 for the fissure flow model (generalized) ..................79

Figure 39. Times 1, 2, and 3 for the multiple dip-slip thrusting model (generalized). ....82

Figure 40. Times 1.2.3. and 4 for the basalt exhumation model ...................... 44 


\section{List of Tables}

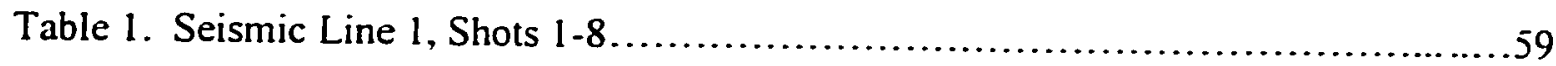

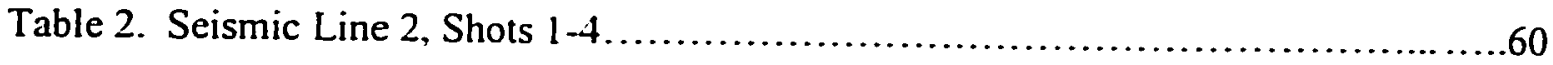

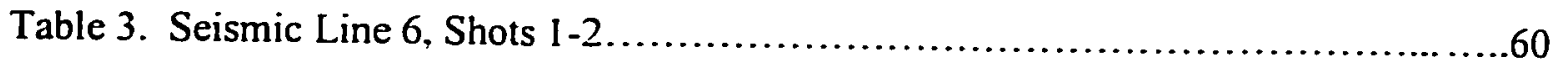

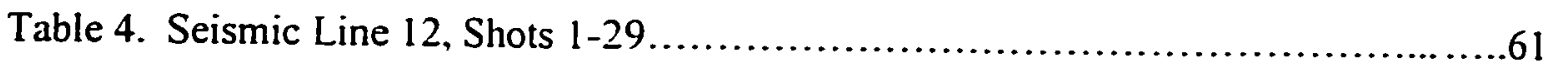

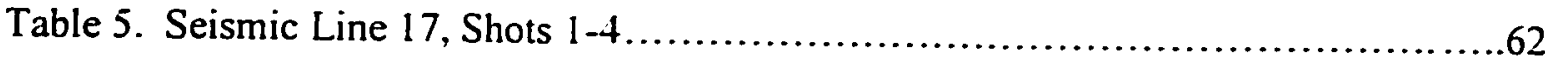

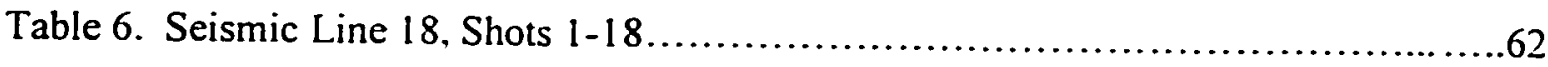

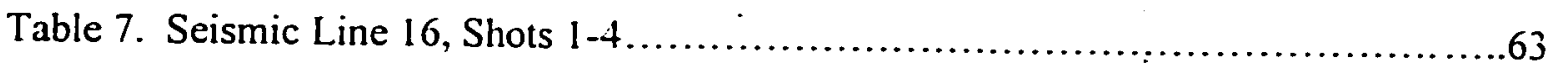

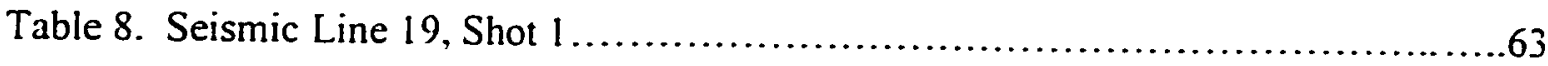

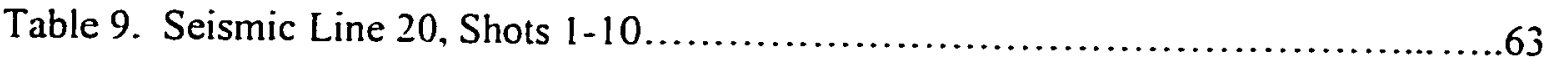

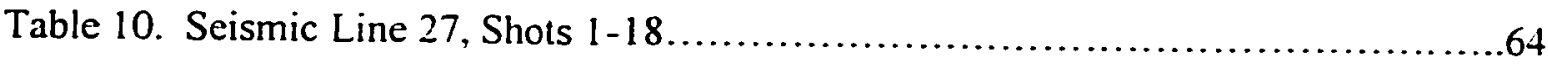

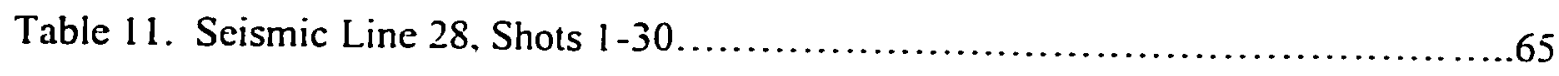

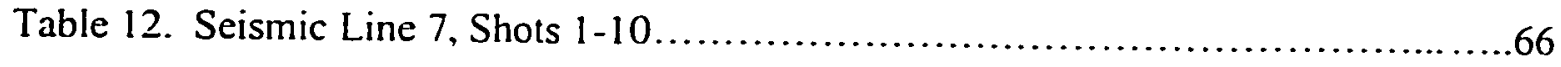

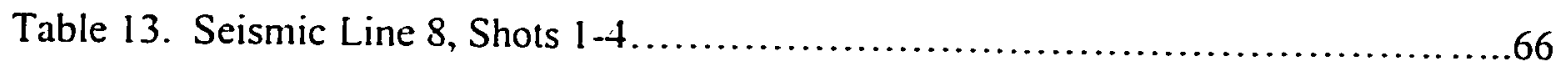

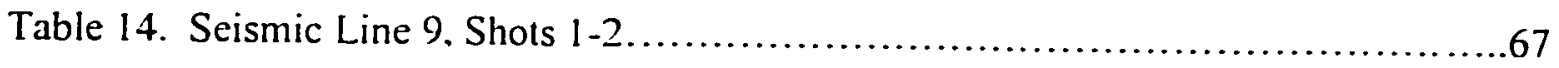

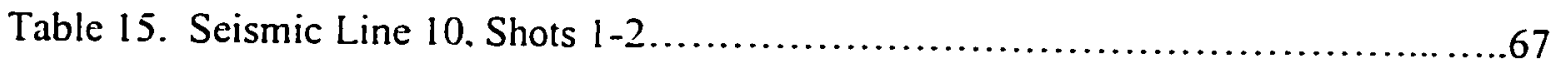

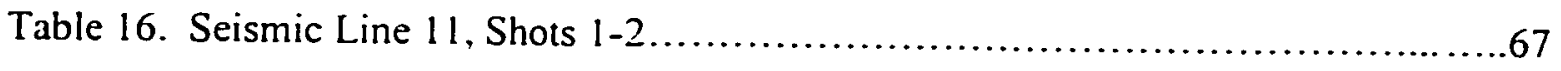

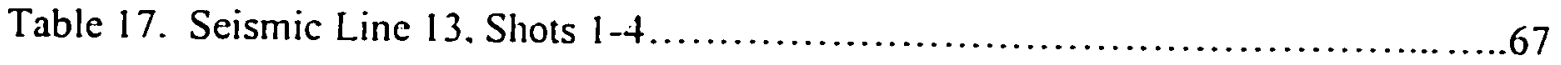

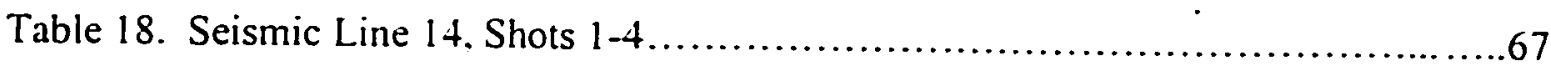

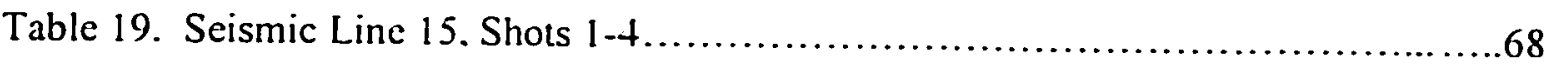

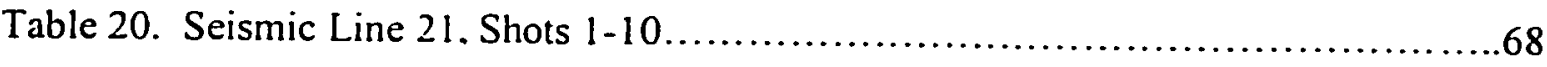

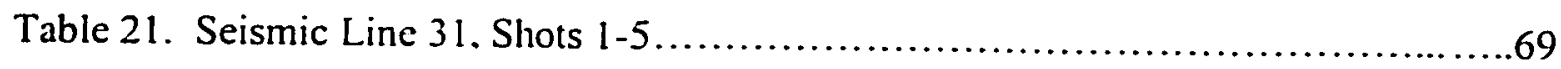




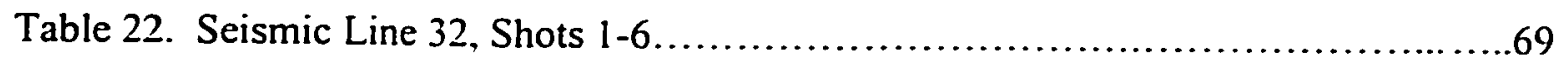

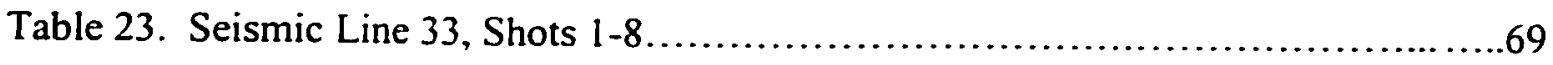

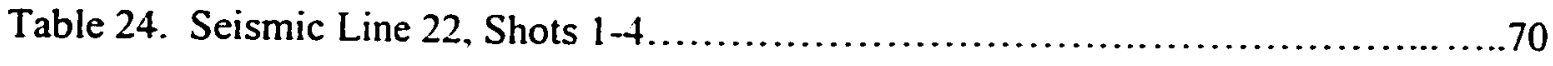

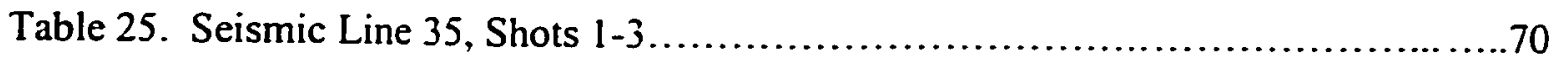




\section{INTRODUCTION}

The 1992 M7.3 Landers and the 1999 M7.1 Hector Mine earthquakes underscored the need for a better understanding of earthquake recurrence and fault kinematics in the eastern California shear zone (ECSZ) of southern California. In direct response to this need, this report investigates evidence for previous activity on the Lavic Lake fault, which produced the Hector Mine earthquake of October 16, 1999 in the Mojave Desert of southern California.

The main objectives of this study are to investigate and identify evidence of previous activity on the Lavic Lake fault. This evidence primarily includes surface and subsurface basalt geomorphology, and is investigated through seismic refraction surveys, magnetometer surveys, and detailed Global Positioning Systems and Geographic Information Systems (GPS/GIS) mapping. This study then presents kinematic models that may not only explain the local geology but also give some clues about previous major earthquake activity on the Lavic Lake fault and neighboring fault systems. 


\section{LOGISTICS}

Lavic Lake is located entirely within Twentynine Palms Air-Ground Combat Center, a Marine Corps training facility in active operation since World War II. Its remote location makes it an ideal locality on which to hold training exercises, controlled bombing runs, and tank driver training. For security and safety reasons, unauthorized civilian access to the base is illegal. Access to the base is allowed only during brief "windows of opportunity" when military exercises are not scheduled.

Any civilian wishing to gain access to the base during these windows must first take a brief $(<1$ hour) ordnance and desert safety training from the Marine Corps on the main base in Twentynine Palms. Training addresses the dangers of live or unexploded ordnance, desert survival techniques, and protocol for contacting BEARMAT, the range coordinators. Civilians must work in groups of at least two, and must use a four-wheel drive vehicle. Contact with BEARMAT must be established by cellular phone or radio when entering and leaving the base and at pre-determined intervals of time while on the base. I have received ordnance training, and am authorized to work on the base with anyone who has also received the training. Permission to work and a planned itinerary must be authorized in advance by Karl Gross of the USGS, who relays the request to the Marine Corps in Twentynine Palms.

Previous military activity may affect this study in several ways. Aerial photographs and ground-based work indicate that the field area has been bombed heavily in the past. Impact craters, unexploded shells, and shell debris are widespread, and are especially abundant in the study area. Large explosions may have severely altered the 
geomorphology of basalt or lake sediments, or may have exposed bedrock or other features in the near subsurface. Ordnance and scrap metal that can be seen on the surface, and that perhaps are buried in the young lake sediments, may be a source of error in the magnetometer surveys. Since Lavic Lake is located at the northern end of the base, ordnance collection teams visit Lavic Lake relatively infrequently, allowing scrap metal to accumulate. A further discussion of errors can be found in the "Error Analysis" section. 


\section{GEOLOGIC SETTING}

\section{The Mojave Block and the Eastern California Shear Zone}

The study area is at Lavic Lake, a playa in the Mojave Desert, southern California west of the Bullion and Cady Mountains (Ross, 1992). The playa is located near the center of the Mojave block, which is bounded by the Garlock fault to the north and the San Andreas fault to the southwest, among other fault systems in southern California. The Mojave block is cut by the ECSZ, in which deformation is dominated by northnorthwest-trending right-lateral strike-slip faults, mostly Cenozoic in age (Fig. 1) (Jagiello et al., 1992). Collectively, the closely spaced faults of the ECSZ accommodate $13-14 \mathrm{~mm} / \mathrm{yr}$ of dextral shear (Miller et al., 2001).

\section{Quaternary Geology}

All surficial units in and near Lavic Lake playa are Quaternary in age (Fig. 2) (Dibblee, 1966). The playa is bounded to the north by flows that originated from Pisgah Crater, and to the west by basalt flows that probably originated from Sunshine Craters. Two small, isolated basalt bodies crop out near the western edge of Lavic Lake playa. These bodies are called the "hand" and the "frisbee." due to their shape. The Sunshine Craters flows, along with the hand and the frisbee, are similar in lithology to the Pisgah flow - fine-grained, vesicular, massive basalt with sparse olivine phenocrysts (Dibblee, 1966) (Figs. 3-6). The lake sediment was characterized by Dibblee (1966) as coarse- to fine-grained alluvium and clay. Field observations indicate that sedimentary units range from extremely poorly sorted gravel to well-sorted fine clay deposits that are prone to 


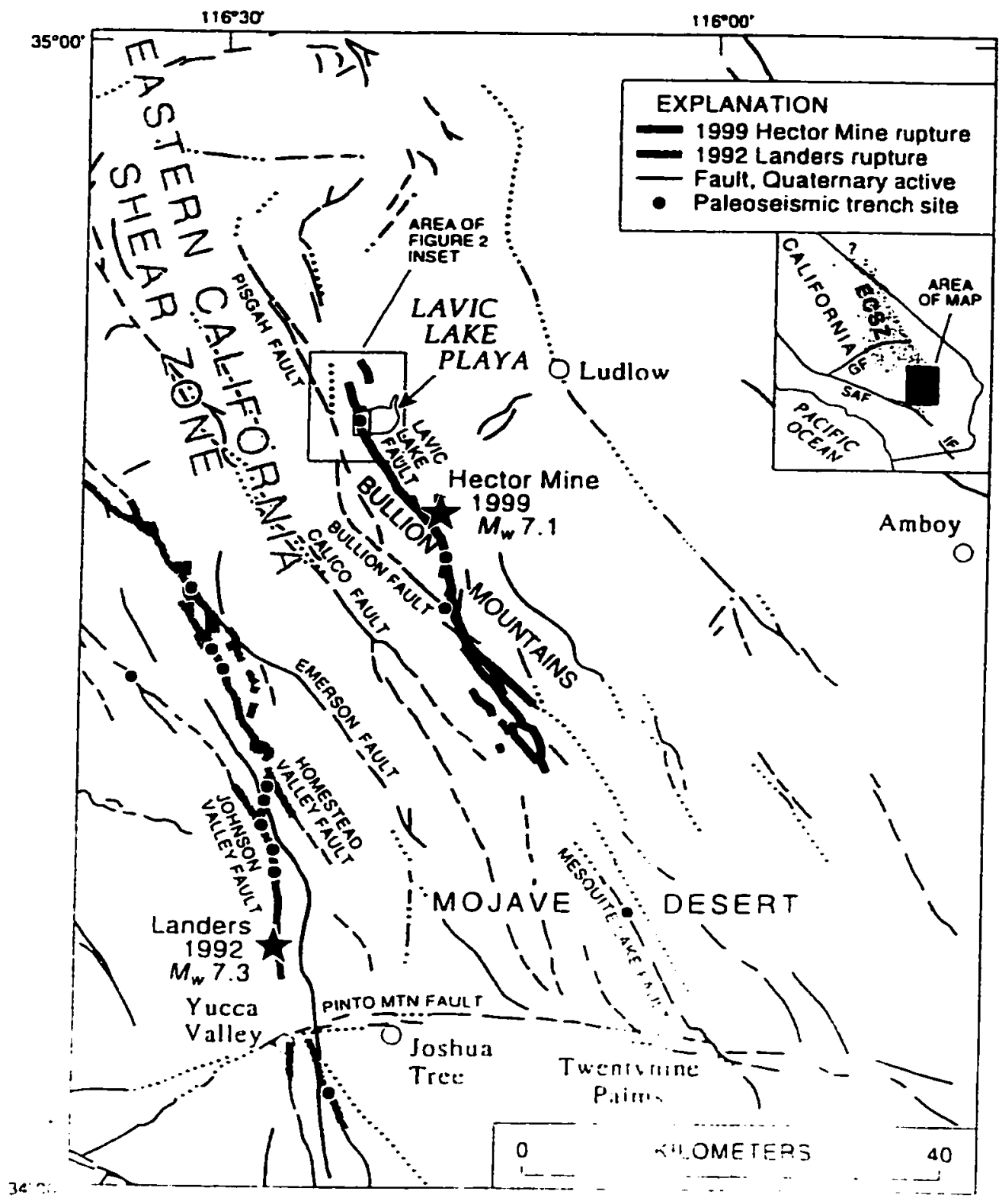

Figure 1. Generalized map showing active faults of the southeastern Mojave tectonic block. The location of Lavic Lake is shown. and the heary black line shows the location of the 1999 Hector Mine earthquake surface rupture. Map modified from Rymer et al. (2002). 


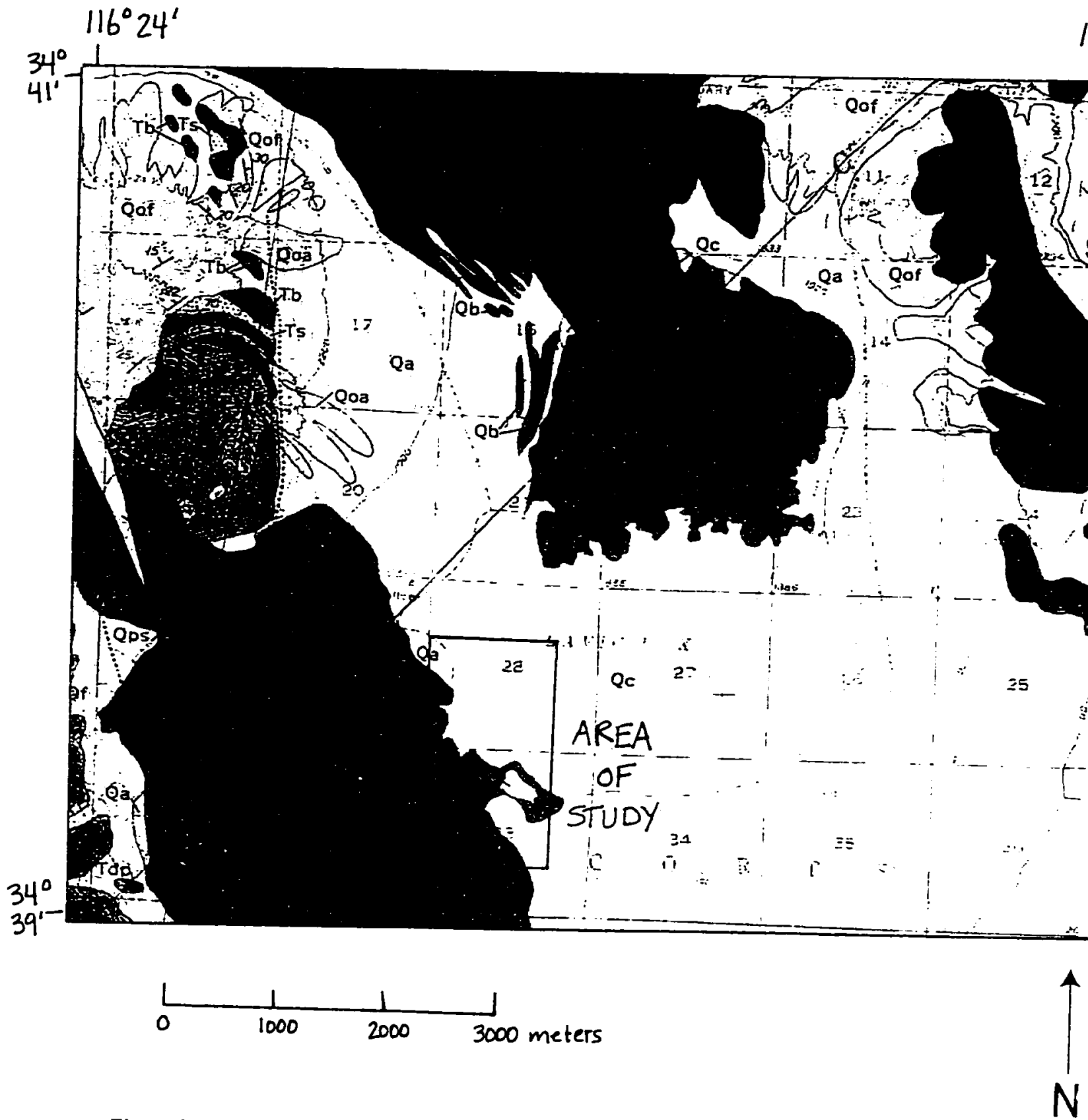

Figure 2. Generalized geologic map of the Lavic Lake area. Key: $Q_{b s}=$ Basalt of the Sunshine Crater flow and craters, $Q_{b}=$ Basalt of the Pisgah flow and crater, Surficial sediments: $Q_{c}=$ clay, $Q_{z}=$ alluvium. Map from Dibblee (1966). 


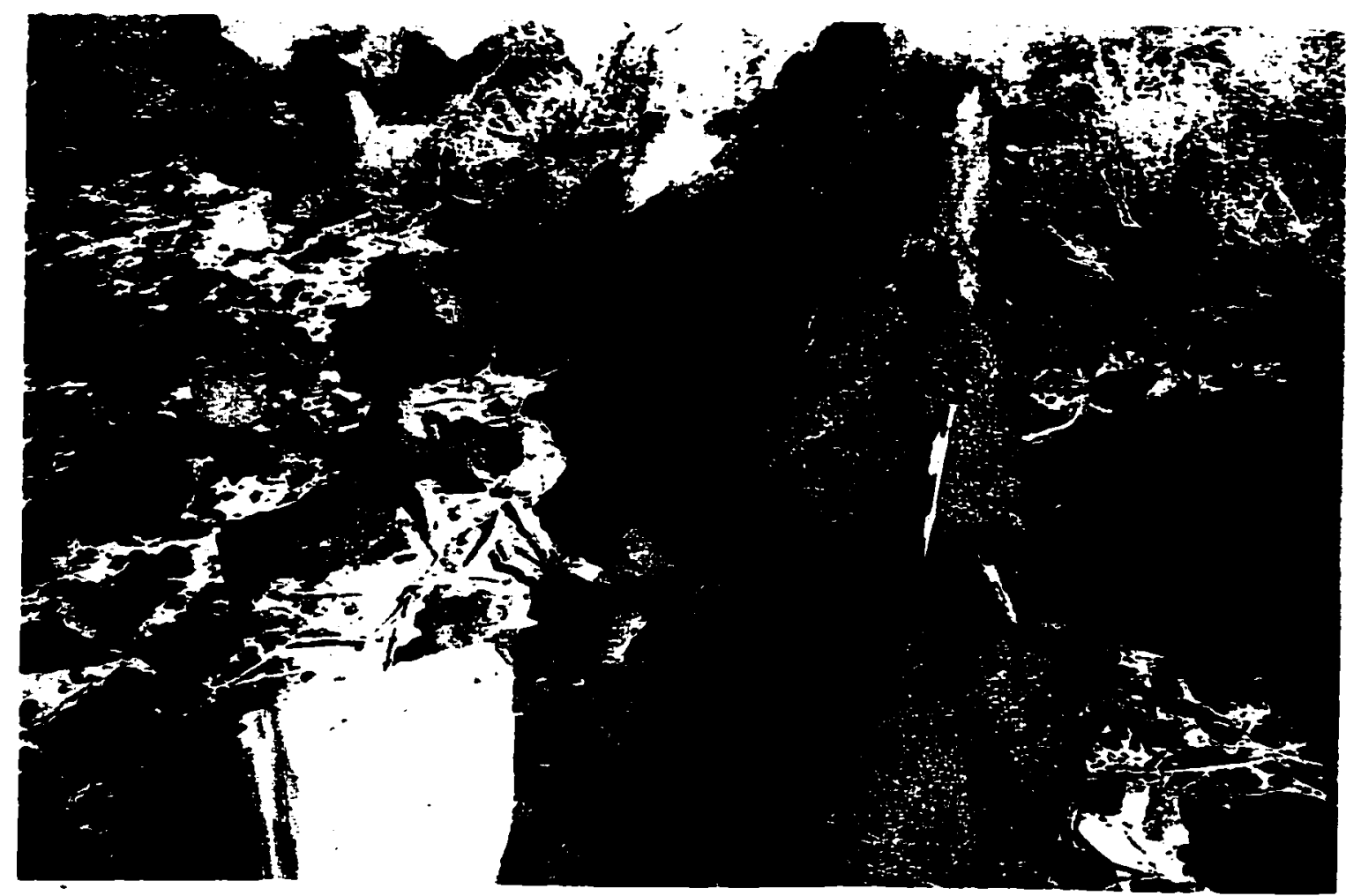

Figure 3. Photograph of surficial basalt units found on the northern tip of the hand in Lavic Lake playa. The basalt is massive but altered by eolian weathering. Pencil for scale. 


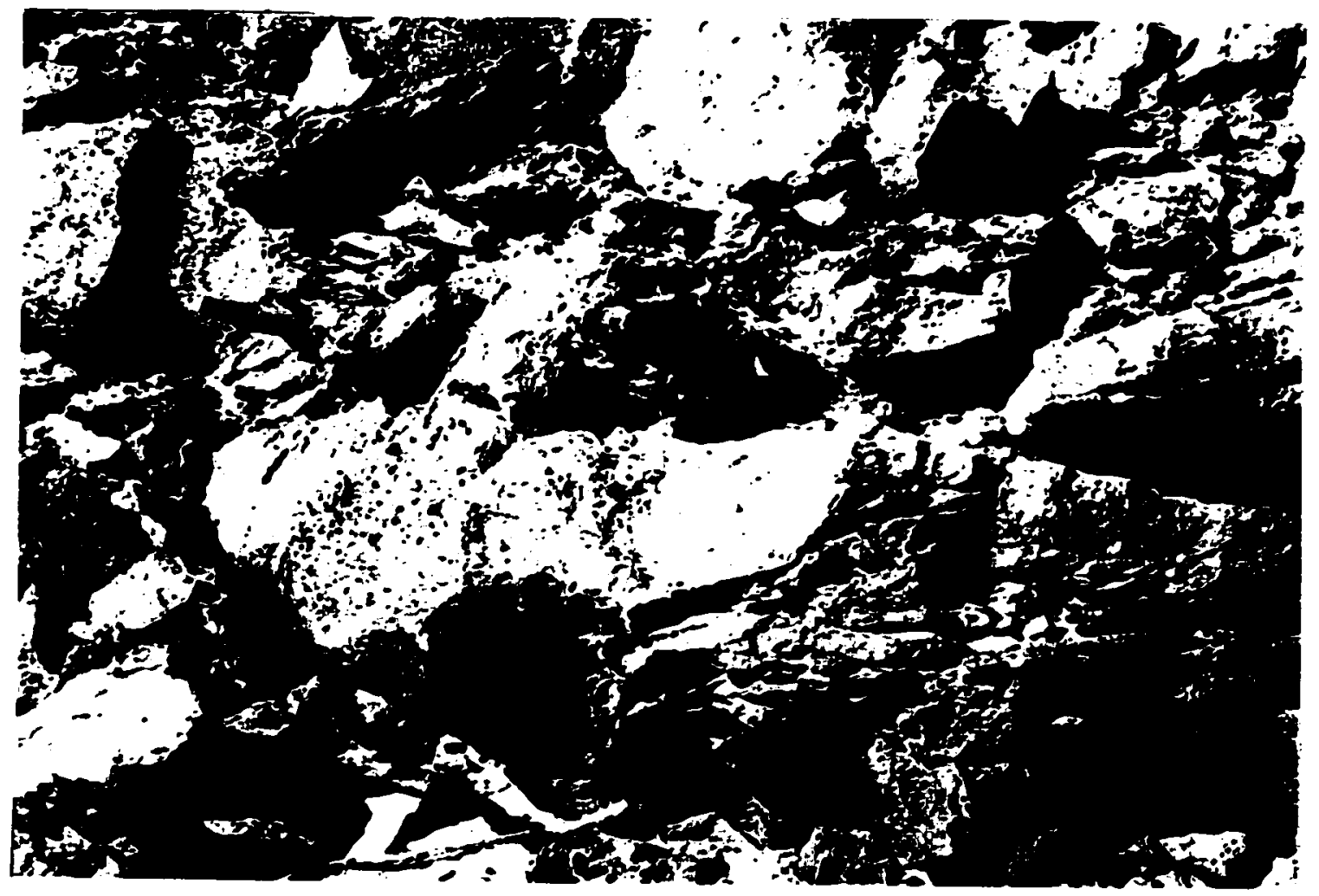

Figure 4. Photograph of surficial basalt units found within the hand near the Hector Mine earthquake rupture. Prominent fracturing is evident. Pencil for scale. 


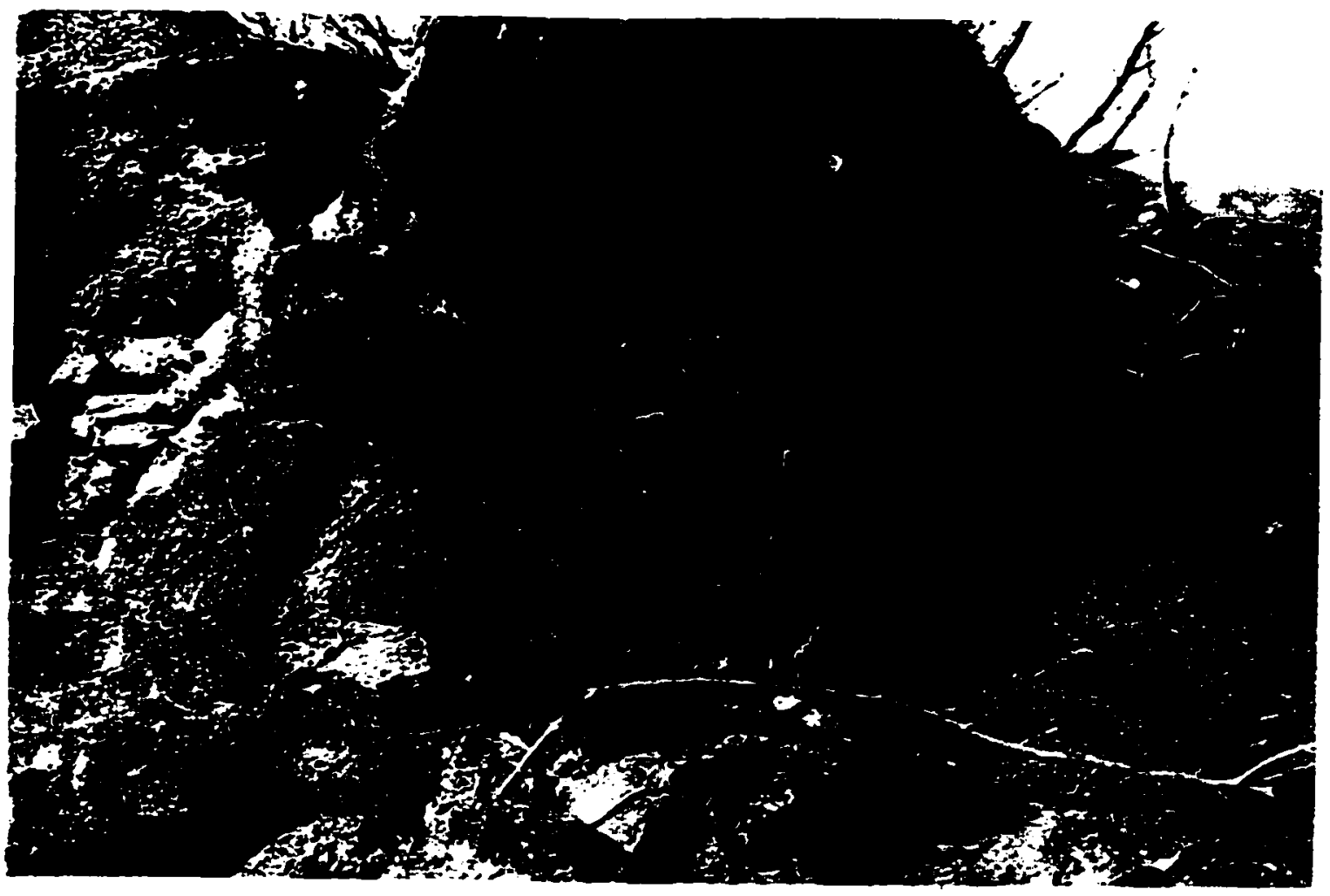

Figure 5. Photograph of in-place surficial basalt units. Prominent fracturing is evident. Pencil for scale. 


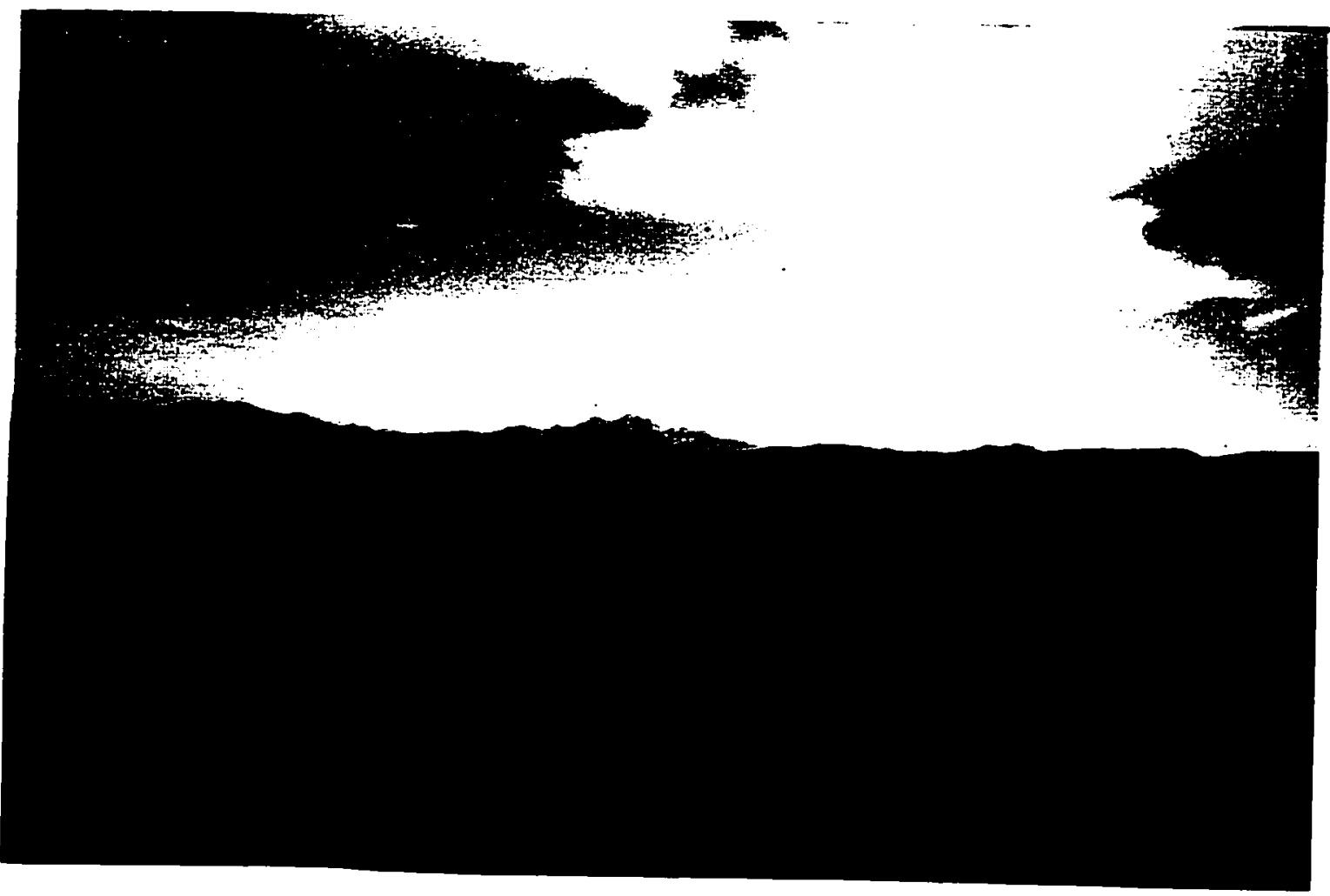

Figure 6. Photograph of flow basalt in the hand, close to the elevation of the sand and clay units of Lavic Lake playa. The impact crater found in the right foreground is approximately $1.5 \mathrm{~m}$ across. 
desiccation cracking (Figs. 7-9).

The age of the basalt flows within Lavic Lake is the subject of some debate, but

all studies agree that the units are late Quaternary. The age of the Pisgah flow has not been determined. The age of the Sunshine Peak flow has been determined (through K-Ar dating) by two researchers. In 1993, John Nakata of the US Geological Survey (USGS) obtained dates of $0.36 \pm 0.1 \mathrm{Ma}$ and $0.26 \pm 0.1 \mathrm{Ma}$ (A. Glazner, personal communication, 2000). Brett Cox of the USGS dated the basalt at 0.60 Ma (A. Glazner, personal communication, 2000). It is unknown whether an attempt was made to delineate separate flows within the basalt units.

\section{Faulting and Current Seismicity}

At 2:46 am PST (0946 GMT) on October 16, 1999, a magnitude M7.1 earthquake occurred on the Lavic Lake fault, an unnamed fault of the ECSZ. The epicenter of the event was located about $76 \mathrm{~km}$ east-southeast of Barstow, California, within Twentynine Palms Air-Ground Combat Center. This earthquake, named the Hector Mine earthquake. produced a spectacular surface rupture with a total length of $48 \mathrm{~km}$ (Treiman et al.. 2002). Portions of the Bullion fault to the southwest and at least four other faults ruptured coseismically. The trace of surface rupture along the Lavic Lake fault is clearly expressed in the sediments and basalt of Lavic Lake playa (Figs. 10-12).

Lavic Lake and the surrounding areas host many other named and unnamed strike-slip faults, all part of the ECSZ (Fig. 13). The Pisgah fault has a visible step in the Sunshine Peak lava flow about $2 \mathrm{~km}$ west of the Lavic Lake fault (Fig. 14). Farther west are the Bullion, Calico, and Emerson faults, all of which are defined as active. The 


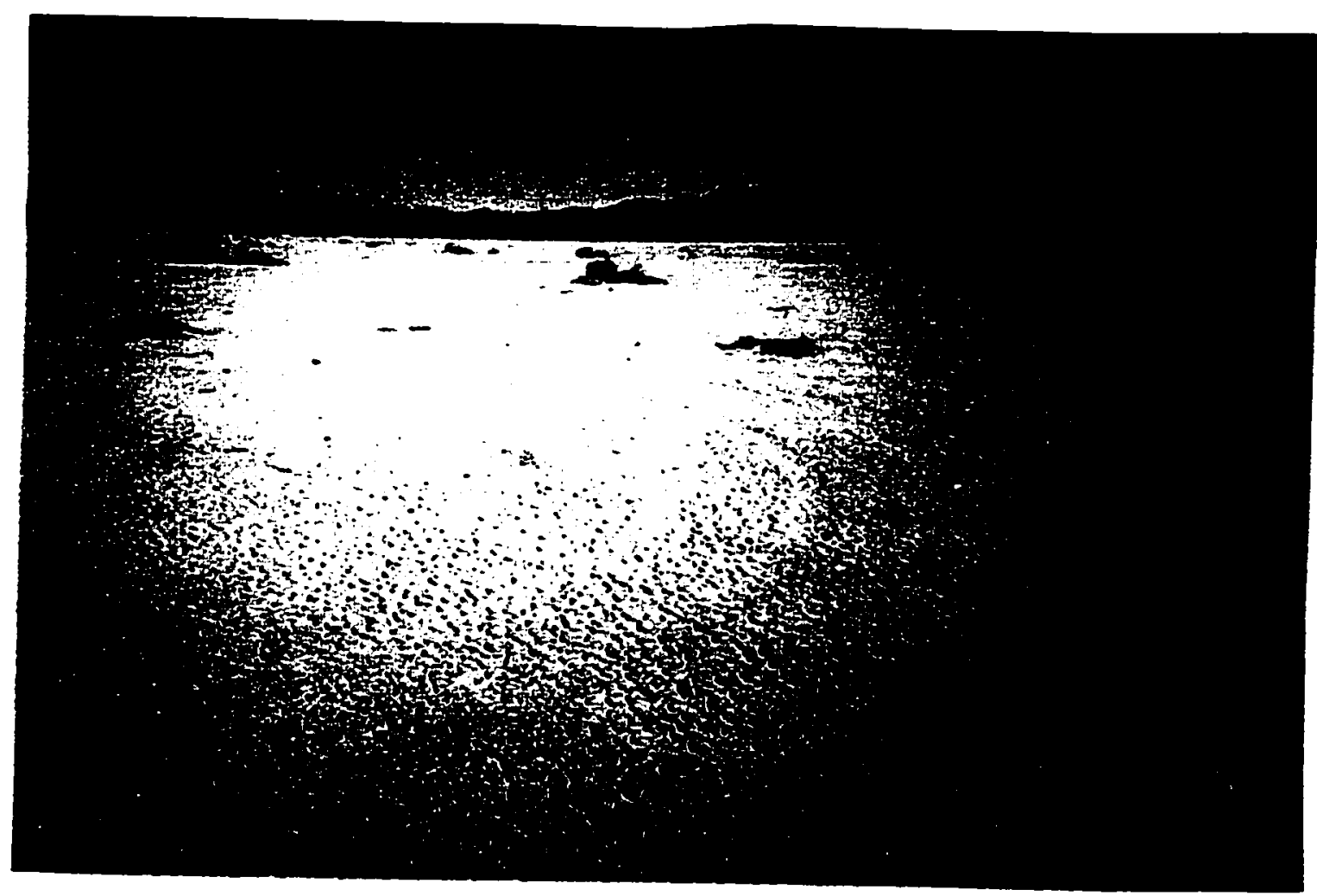

Figure 7. Photograph of surficial clay and sand units that compose most of Lavic Lake playa. Photo was taken facing to the north. Truck tracks for scale. 


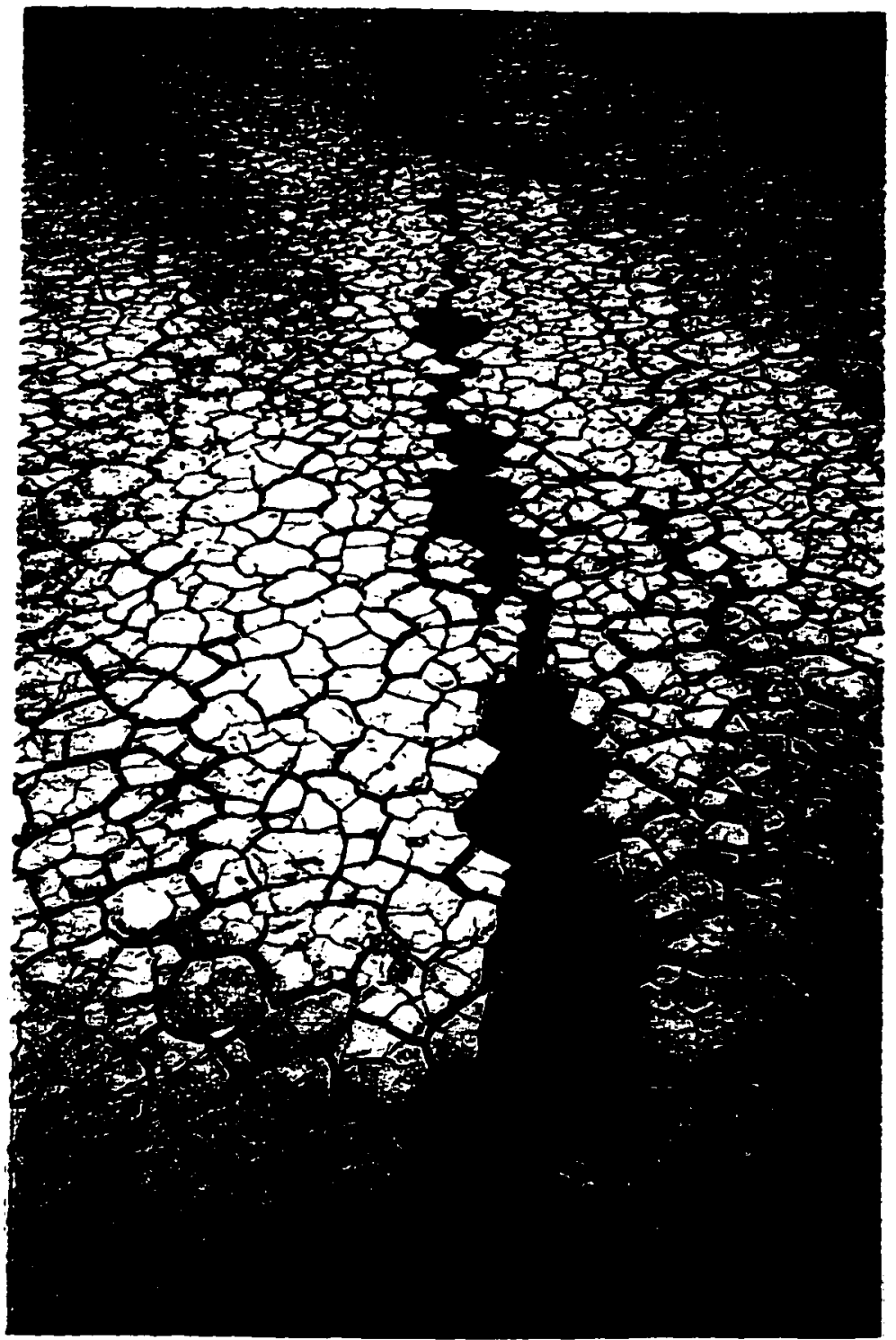

Figure 8. Photograph of surficial clay units, with minor, shallow polygonal desiccation cracks and open fissure. The fissure formed from movement on the Lavic Lake fault. Maximum width of fissure is approximately $0.2 \mathrm{~m}$. 


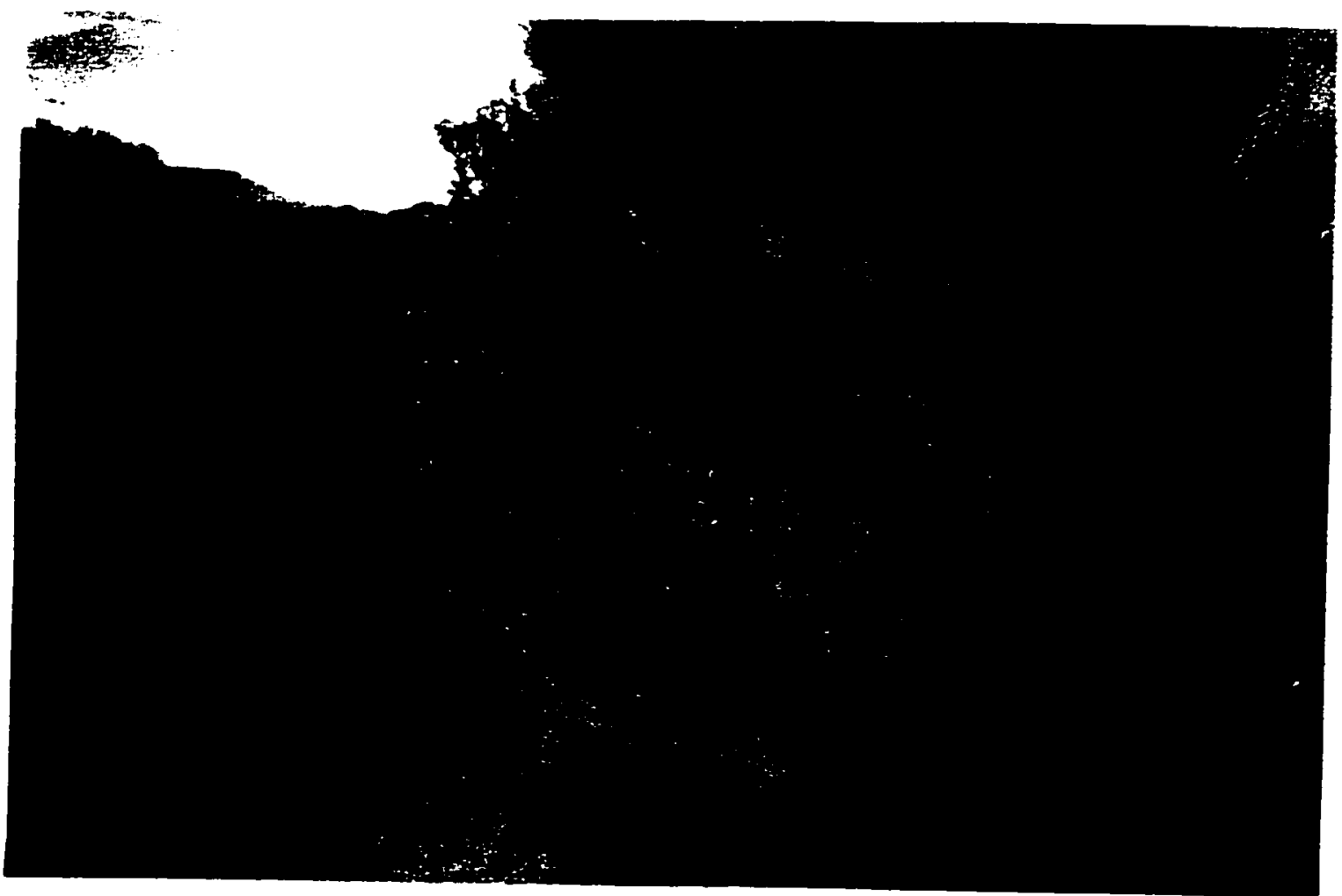

Figure 9. Vertical section of playa exposed due to the Hector Mine earthquake. Pencil for scale. 


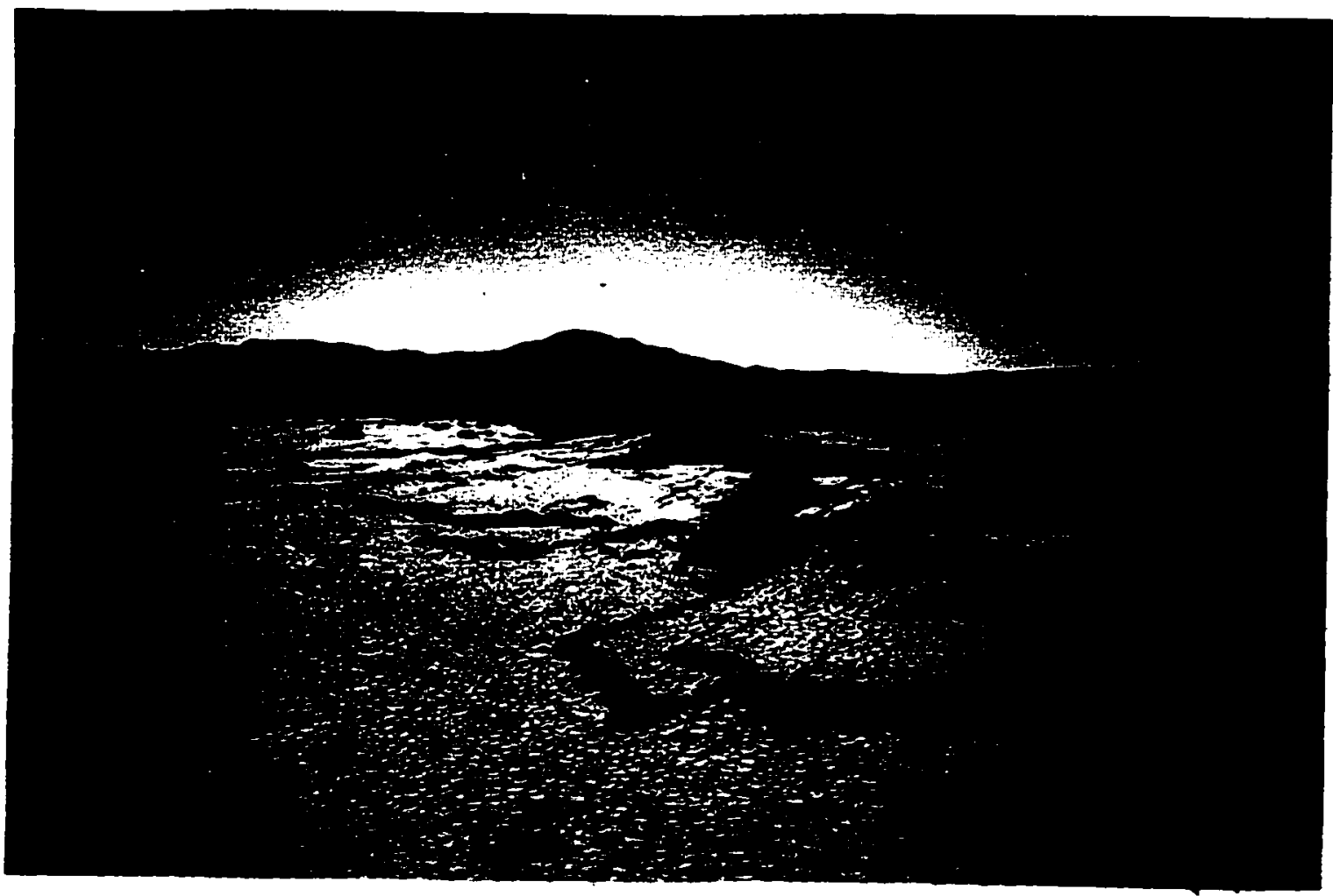

Figure 10. Section of rupture between the hand and frisbee. Photograph taken looking to the south. Compression due to right-lateral movement is shown by playa sediment "sheets" thrust towards the observer. Bushes are approximately $1.0 \mathrm{~m}$ across. 


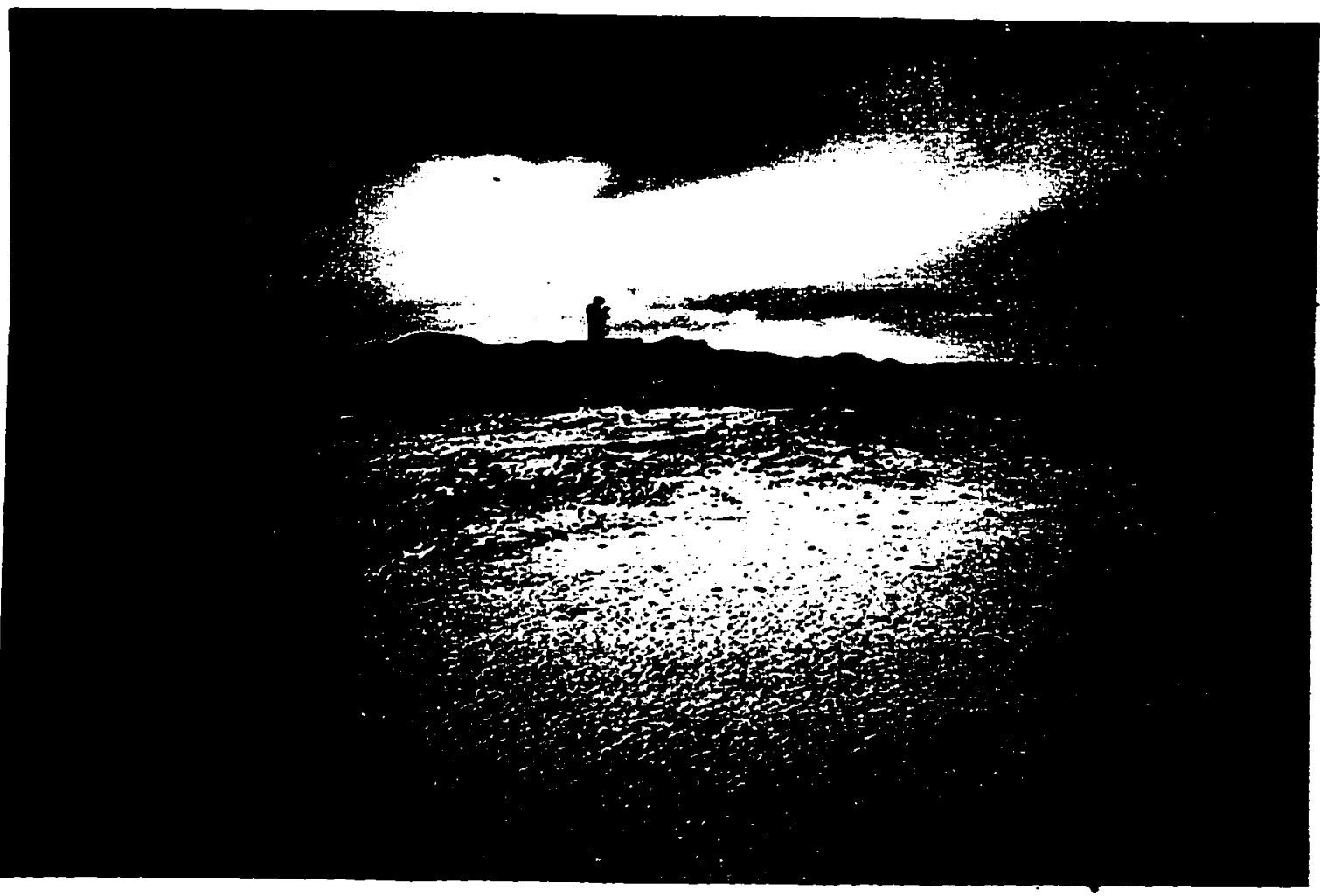

Figure 11. Section of rupture cutting through the frisbee. Photograph taken looking to the northwest. Vertical relief is due to minor east-vergent thrusting. Person is $2.0 \mathrm{~m}$ tall. 


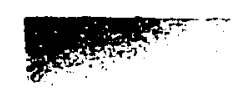

Figure 12. Section of rupture south of the hand. Photograph taken looking to the southeast. Bushes are approximately $1.0 \mathrm{~m}$ across. 


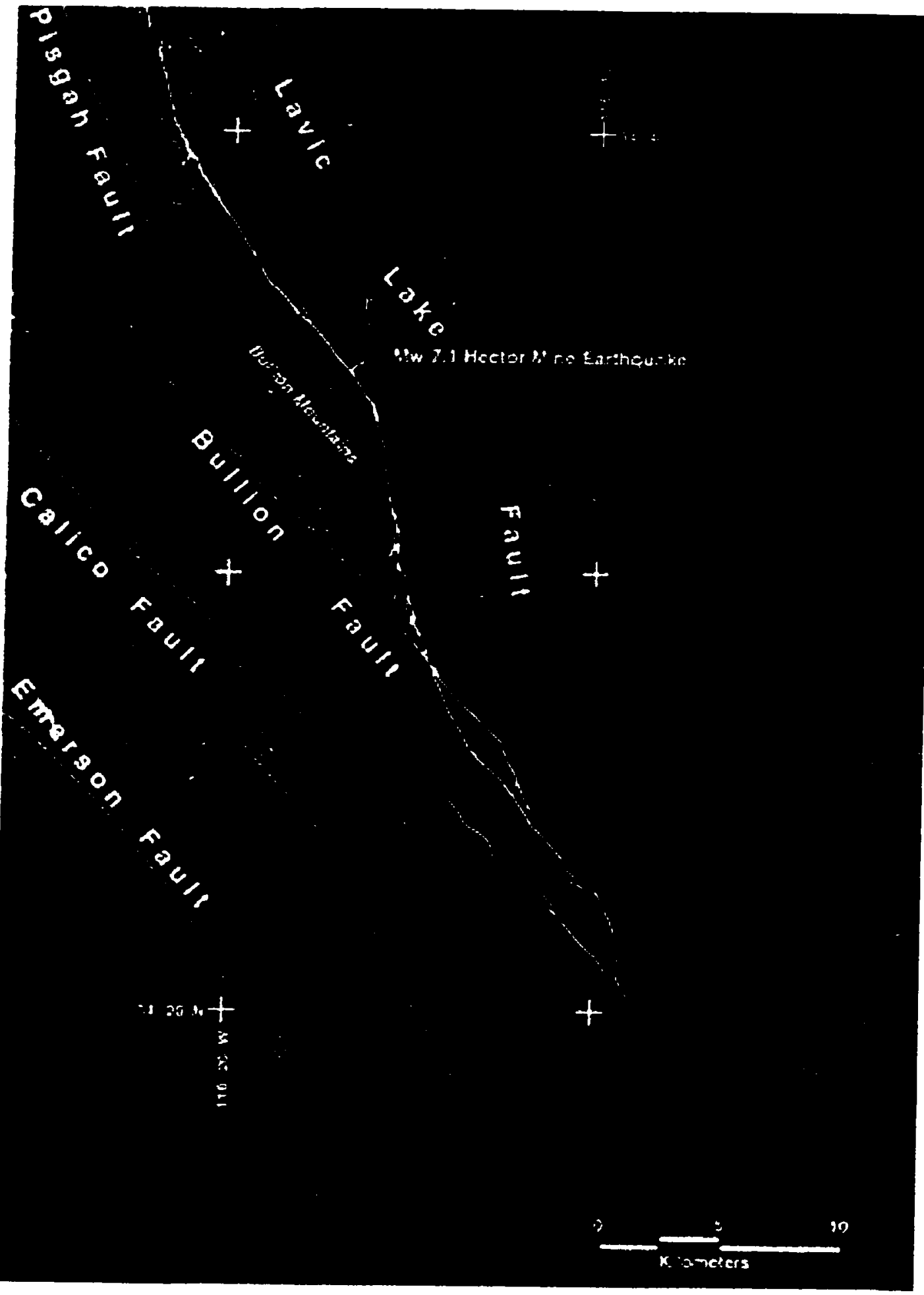

Figure 13. Major strike-slip faults in the area of Lavic Lake, with the principal 1999 Hector Mine rupture shown in yellow. Map from USGS, SCEC, and CDMG (2000). 


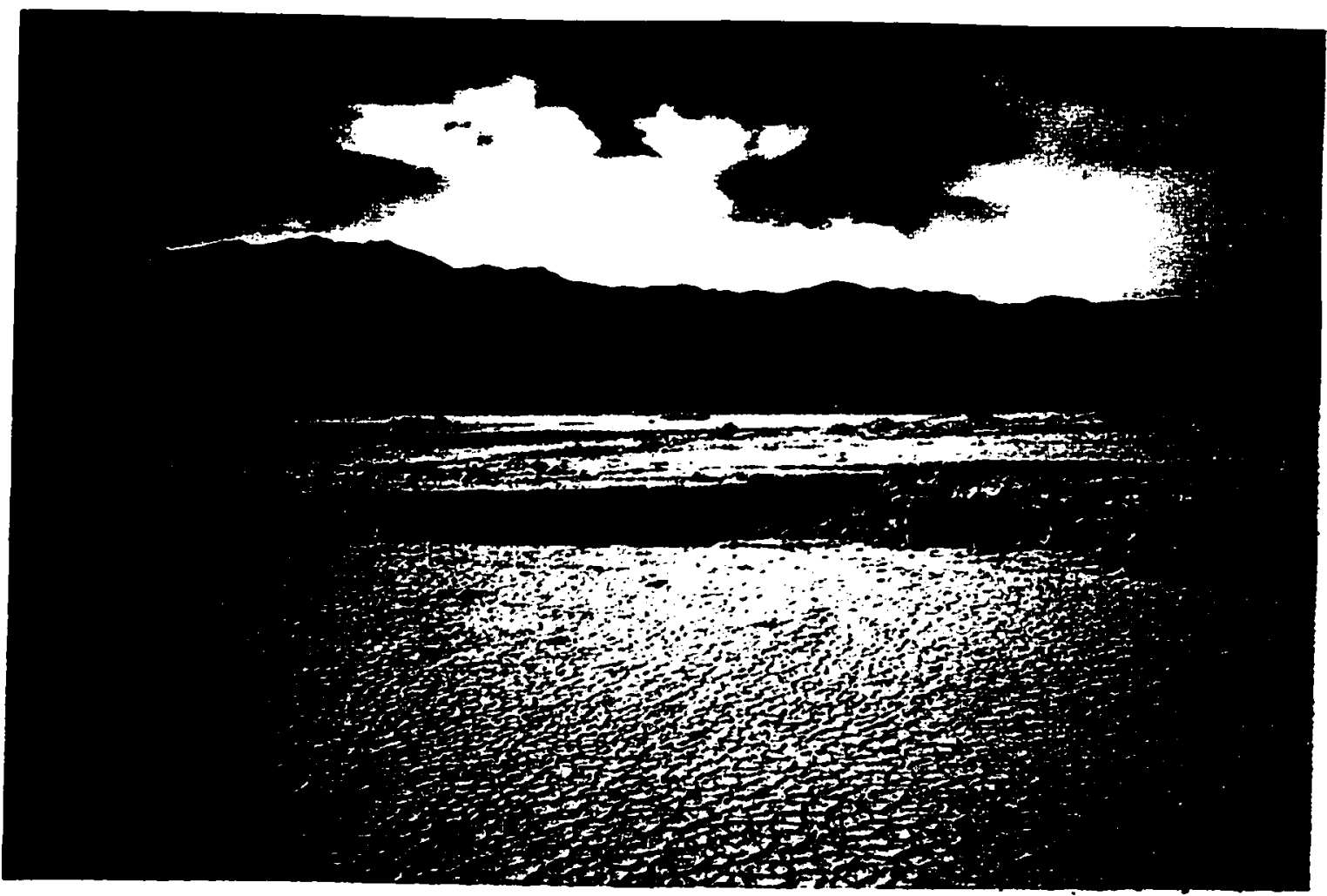

Figure 14. Photograph of portion of the Lavic Lake fault rupture and the Pisgah fault offset, about $2 \mathrm{~km}$ to the west. Photograph taken looking to the west. Pink " $X$ " in the foreground is apprixmately $0.3 \mathrm{~m}$ in length and width. 
Bullion fault intersects the Lavic Lake fault southeast of Lavic Lake in a 700 - $\mathrm{m}$-wide zone of deformation made evident by the Hector Mine earthquake (USGS, SCEC, and CDMG, 2000). The close spacing of all of these faults suggests that they may be related seismogenically or have the ability to transfer stress from one fault to the next. All of these faults have very low slip rates, suggesting very long recurrence intervals (USGS, SCEC, and CDMG, 2000). 


\section{PREVIOUS WORK}

The geology of the study area was mapped by Dibblee (1966) and Wise (1966). Since the 1992 M7.3 Landers and the 1999 Hector Mine earthquakes, the region has been the subject of renewed geologic interest to many researchers. However, much of this research is not yet published.

On the day of the Hector Mine earthquake, helicopter reconnaissance flights identified and mapped the entire surface rupture. Aerial photographs of the rupture at 1:10,000 scale were taken two days later, after military operations ended. During fieldwork, geologists measured offsets at more than 300 locations along the entire fault rupture. All of this work was a collaboration of the USGS, the Southern California Earthquake Center (SCEC), and the California Division of Mines and Geology (CDMG) (USGS, SCEC. and CDMG, 2000).

Besides the documentation of the surface rupture days after the earthquake, further work was done to study the fault in more detail. In April 2000, trenches were dug at three locations on the surface rupture in Lavic Lake playa, for the purpose of paleoseismic studies (Rymer et al., 2002). Photogrammametric surveys were also taken by Michael Rymer near stepovers on the surface rupture, and some precise mapping data were collected with a total station. 


\section{BACKGROUND AND PURPOSE}

The Landers and Hector Mine earthquake sequences provide opportunities to gain a greater understanding of the fault histories and recurrence intervals of major earthquakes within the ECSZ. Moreover, geomorphic features of the rupture in westem Lavic Lake playa are amenable to the use of field techniques that complement traditional paleoseismic studies.

This study concentrates solely on the western edge of Lavic Lake, where the Lavic Lake fault ruptured directly through isolated outcrops of basalt, the hand, and the frisbee (Fig. 15). The presence of basalt along the fault rupture may indicate that the hand and the frisbee are somehow fault-related. Moreover, USGS scientists identified “ancient. subdued fault scarps along the 1999 [Lavic Lake fault] rupture zone," implying that the fault zone may have an extensive rupture history (M. Rymer, personal communication).

Below are three groups of questions addressed by this study and a general description of the techniques used for each.

\section{A. The basalt-sediment contact}

1. What is the depth of the basalt beneath Lavic Lake playa, specifically west and directly east of the hand? .

2. What is the geometry of the basalt-sediment contact in the subsurface?

3. Are the hand and the main Sunshine Crater flows connected in the subsurface?

4. Which features in the Sunshine Peak flow and the hand are geomorphic (i.e. features consistent with a typical lava flow) and which are fault-related? 


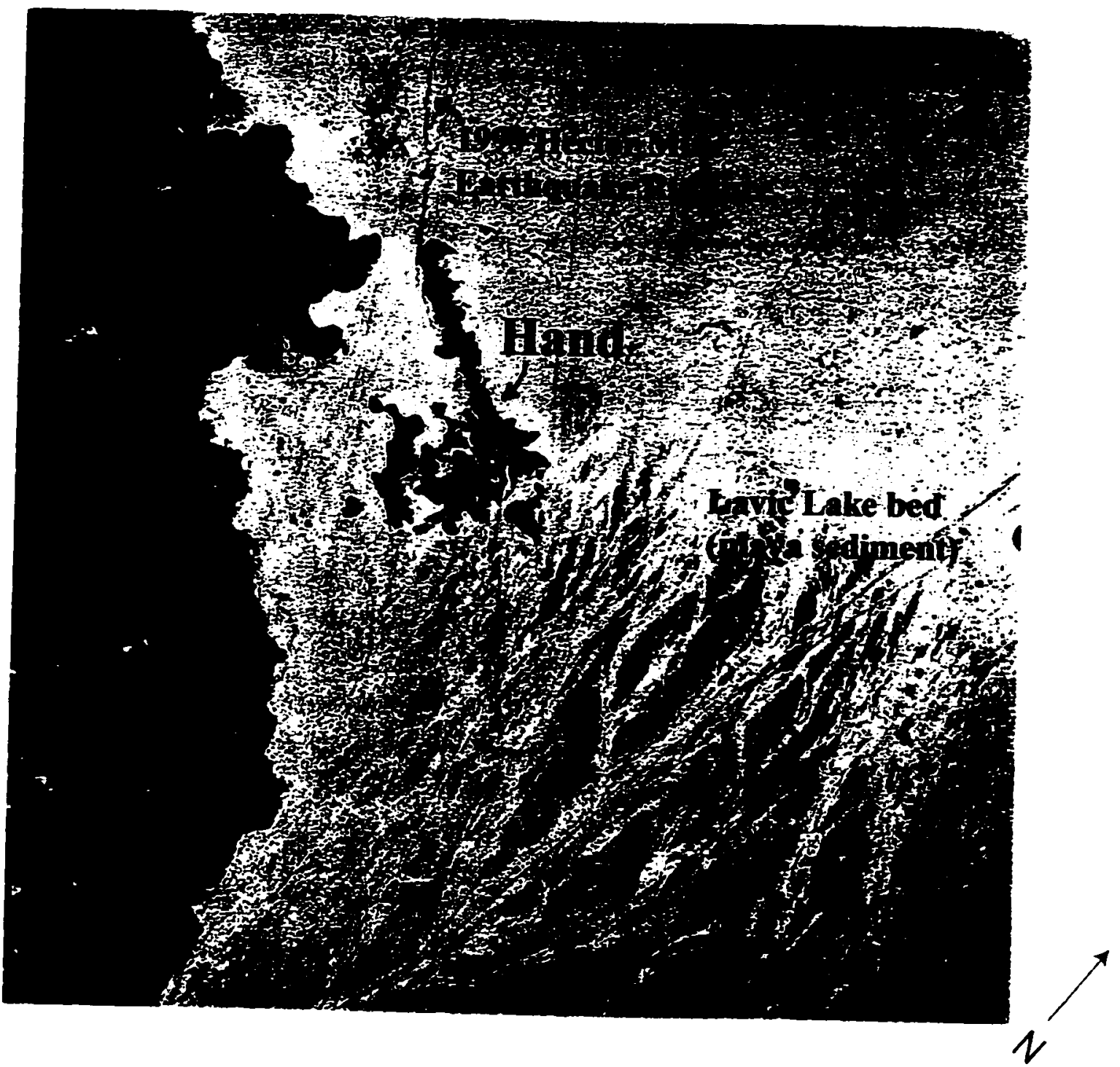

Figure 15. Portion of 1:10,000-scale aerial photograph of study area in Lavic Lake, showing trace of the 1999 rupture. The isolated basalt feature located to the northeast is termed the "hand." The "frisbee" is not shown; it is about $1 \mathrm{~km}$ to the northwest. 
B. The relationship between the 1999 Hector Mine rupture and the surface morphology

5. Can the surface patterns of the Hector Mine earthquake be spatially correlated with the basalt's geomorphic features?

6. Can the patterns of the surface rupture in the hand and frisbee be used to explain why the two isolated features seem disconnected from the main flow?

C. Kinematic models of the current morphology of the hand and frisbee

7. Do patterns indicate or refute a kinematic model in which the hand and frisbee were separated from the Sunshine Peak flow by previous faulting?

Seismic refraction and magnetometer surveys helped address the group $A$ questions. Since only a few unique geologic units are present in the field area, all with differing seismic velocities, seismic refraction can be used to successfully distinguish between basalt, semi-consolidated alluvial material, and near-surface clay, silt, and unconsolidated sand layers. Subsurface structure can be interpreted from such seismic refraction data. Detailed magnetometer surveys can be used to infer depths to mafic basalt bedrock, or possibly to speculate on the shapes or subsurface orientations of this bedrock. Because basalt is the only highly magnetic rock in the field area, a magnetic study may successfully distinguish between basalt and the other units present. Detailed field observations were also used to address question A4.

Groups B and C were addressed most effectively with Differential GPS, which displays spatial data with high accuracy. Geomorphic features can be studied in extraordinary detail on a very fine scale. The GIS can be used to make correlations 
between spatially related features, shedding light on how the Hector Mine rupture and geomorphic features of the basalt flow may be related. Interpolation of the spatial data is necessary to develop a model for how the hand and frisbee may have been affected by previous faulting. 


\section{FIELD METHODS}

\section{Magnetometer Surveys}

Measurements of the magnetic field were taken in the western part of Lavic Lake playa to ascertain the remanent magnetization of basalt structures, the underlying playa sediment, and subsurface basalt (Fig. 16). Measurements were made with a Geometrics portable magnetometer. First and second measurements were taken at a single position. The second measurement was recorded if it differed from the first by less than approximately 0.5 nanotesla $(\mathrm{nT})$. If the difference was greater than about $0.5 \mathrm{nT}$, the measurement was taken a third time, and the third measurement was recorded. If this measurement differed significantly from the others, either no measurement was recorded or the measurement process was started again.

Linear magnetic surveys of the playa were conducted to provide geographic coverage of the study area. The spacing of the magnetometer readings ranged from 10 to 50 feet. If the instrument was unable to yield a readable magnetic field at a particular location, points were chosen 10 feet away in all four compass directions until the field could be measured. Magnetometer lines discussed in this report include lines 1-5, 23-26, 29, 30, and 34 (Fig. 16). In addition to the linear surveys, random points on the playa were also surveyed in order to cover "gaps" that could not be reached by standard linear surveys.

\section{Seismic Refraction Surveys}

Seismic refraction data were collected along linear survey lines of varying lengths and geophone spacing (Fig. 17). Data were collected with a Bison Geopro Model 8012A 


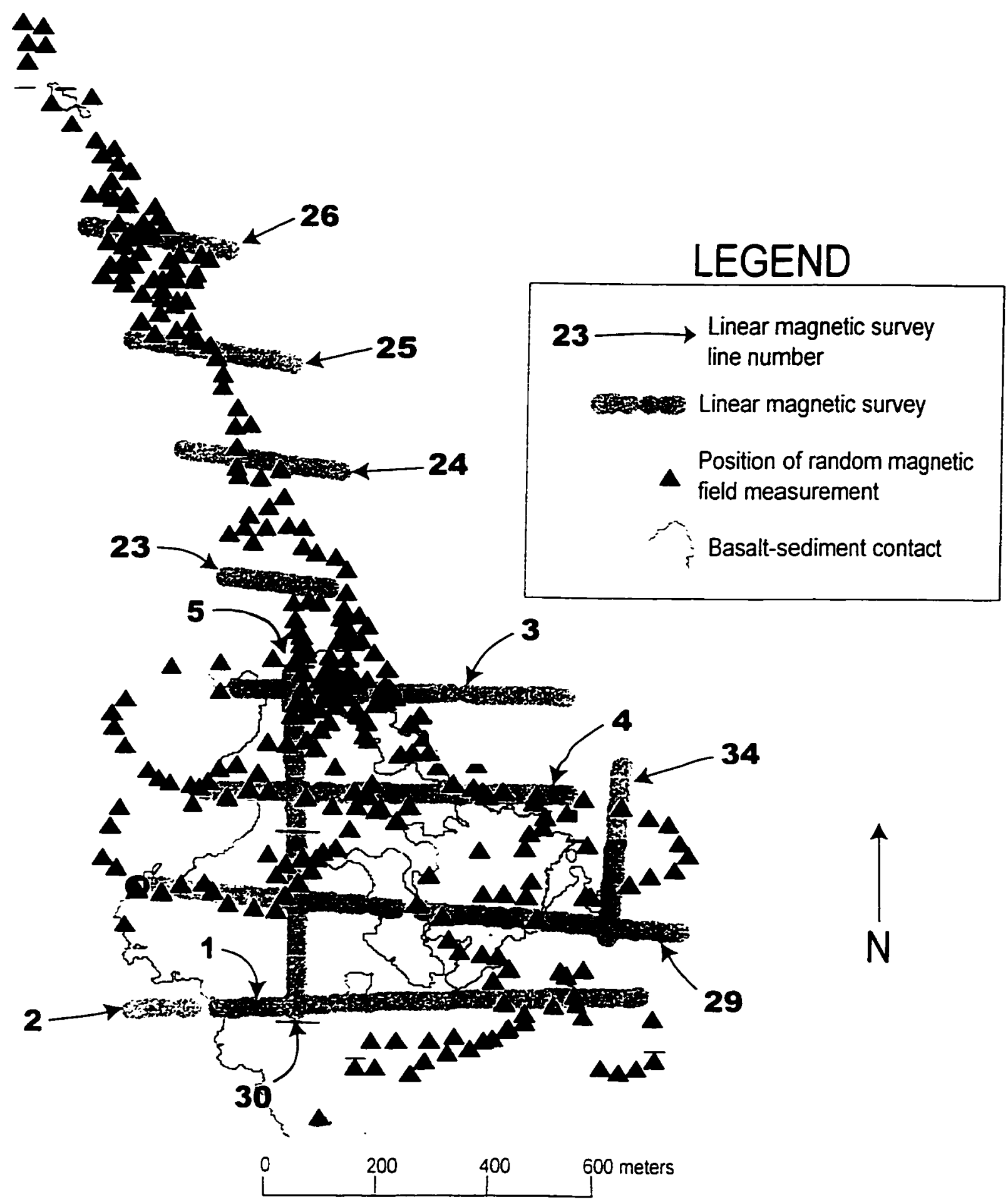

Figure 16. Locations of all magnetometer lines along which data were collected. This figure was generated by spatial data files imported into ArcView $3.2 \mathrm{a}$. 


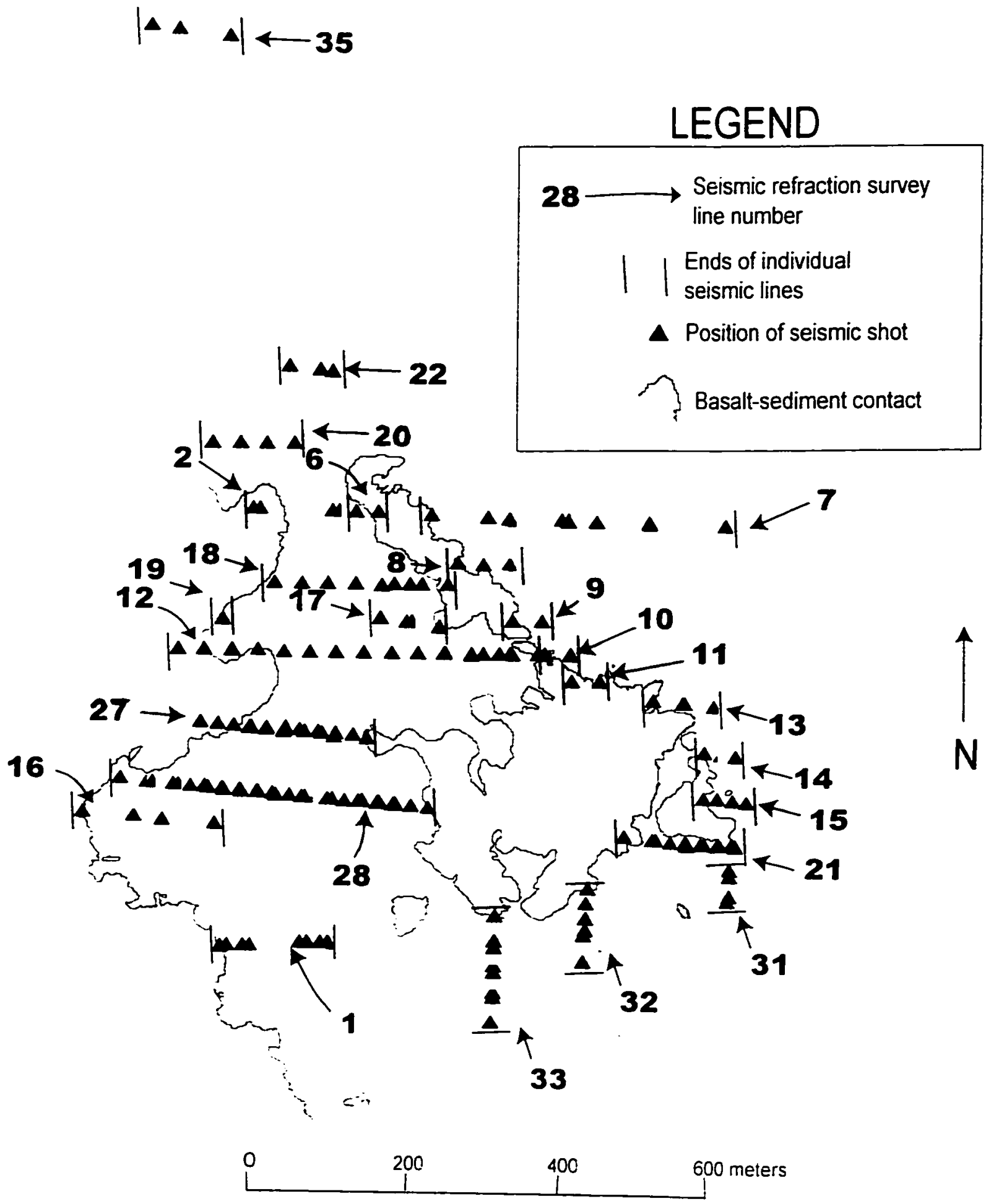

Figure 17. Locations of all seismic refraction lines along which data were collected. This figure was generated by spatial data files imported into ArcView 3.2a. Black triangles indicate the points where the strike plate was hit - connecting those points gives a rough orientation of a seismic line. 
seismic refraction instrument with a cable of 12 geophones (maximum 30-foot spacing) (Figs. 18, 19).

Seismic energy was generated by hitting a strike plate on the ground with a 10-lb. sledgehammer. This sledgehammer was fitted with a trigger, which signaled the refraction instrument to record waves generated by the impact. The strike plate was initially placed at geophone 1 (or 10 feet behind geophone 1) and a "shot" was produced at that point. To test for a dipping layer in the subsurface, the line was reversed by placing the strike plate at the opposite end of the line at geophone 12 (or 10 feet behind geophone 12). Depending on the chosen spacing of the geophones, the reversed shot was either $330 \mathrm{ft}$. or $110 \mathrm{ft}$. from the original shot at geophone 1 . Arrivals of the direct and refracted waves to the seismograph were chosen off the instrument display, and their times (in milliseconds) were recorded.

\section{DGPS/GIS Mapping}

Spatial data in Lavic Lake were collected using a Trimble ProXRS Differential GPS (DGPS) unit. All spatial data collected were differentially corrected to ensure the greatest possible accuracy, typically under \pm one meter.

Using the DGPS unit, a geologic map of the field area was generated to accurately determine the two-dimensional and three-dimensional shape of the hand, the frisbee, and associated surficial geologic units (Fig. 20). The fault rupture caused by the Hector Mine earthquake within the hand and the frisbee was also mapped, along with the locations of every magnetic field measurement, shot point, and other points of interest in the field area. Ample latitude, longitude, and elevation ("x-y-z not in feature") points were 


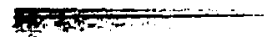

$-$
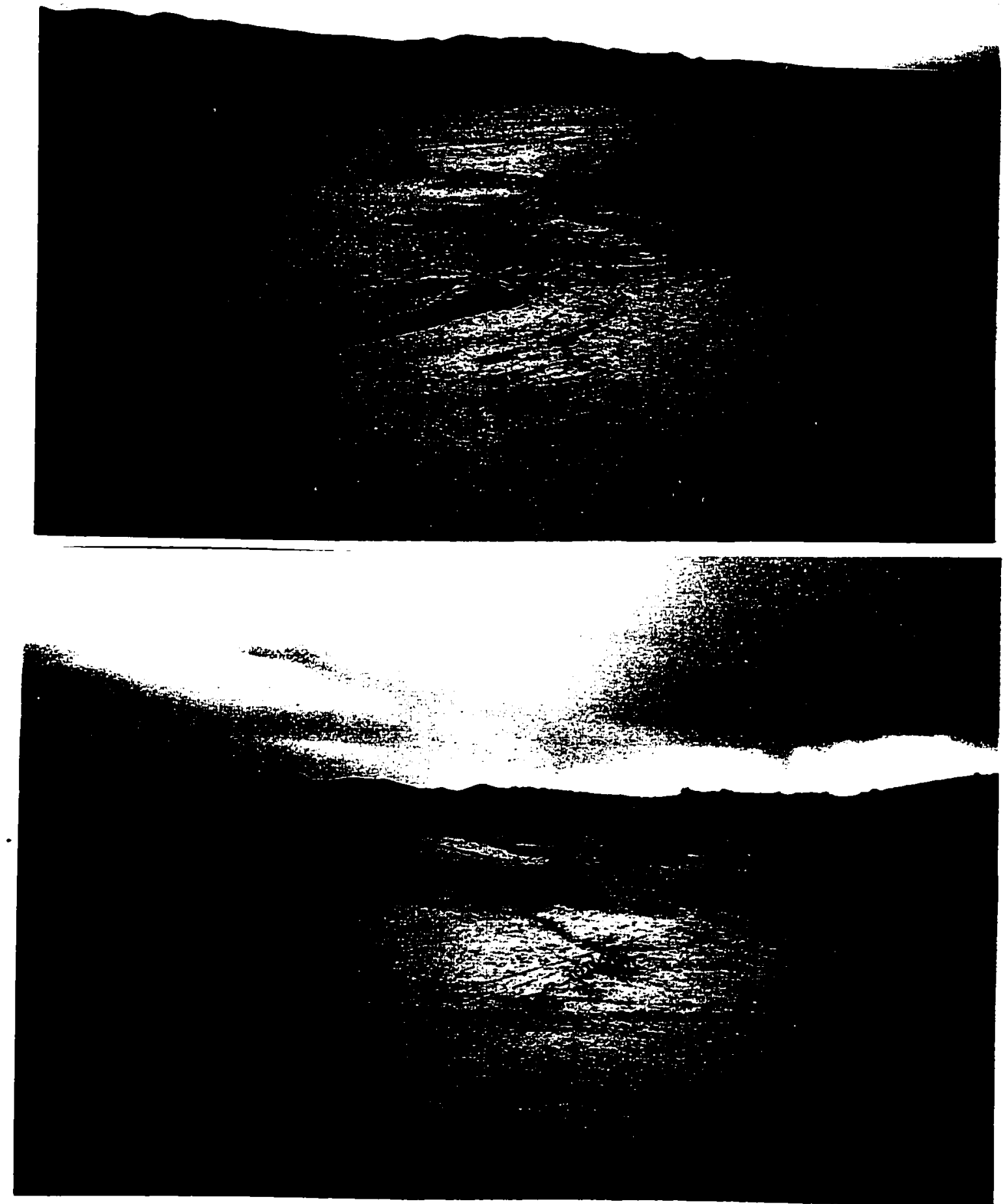

Figures 18 and 19. Photographs of field apparatus for seismic refraction data collection. These seismic lines extend across the Hector Mine rupture, near the north end of the finger. Truck and field assistant for scale. 


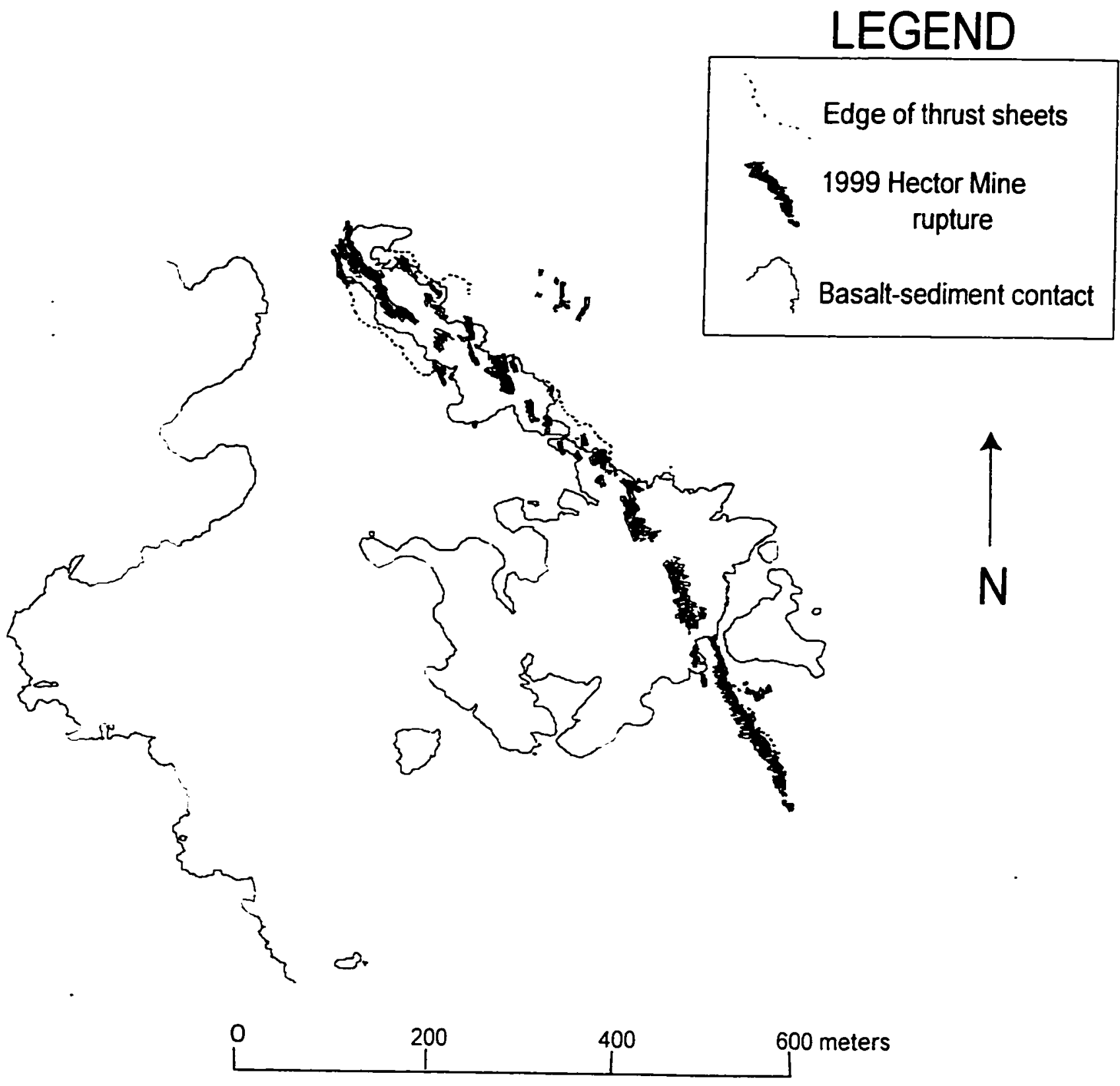

Figure 20. Map of the field area showing the rough spatial relations of the hand, the frisbee, the Hector Mine rupture, and associated units. This figure was generated by spatial data files imported into ArcView 3.2a. 
collected by walking random paths around the field area. These data were exported into ArcView version $3.2 \mathrm{a}$ for spatial analysis, and are best viewed and manipulated with ArcView GIS software. 


\section{EXPERIMENTAL METHODS}

\section{Magnetic Data}

Because each magnetometer reading taken at Lavic Lake playa represents the total induced magnetic field at a specified point, each must be corrected to account for variations in the field due to position (latitude and longitude), elevation, diurnal variations, solar storms, magnetic storms, and the regional trend in the magnetic anomaly.

To correct for position on Earth's surface, or the magnetic field produced by Earth's core, the predicted magnetic field was calculated using the International Geomagnetic Reference Field (IGRF). The field was recently updated for the year 2000, and is termed the IGRF2000 model. The IGRF is produced by geomagnetic field modelers with the International Association of Geomagnetism and Aeronomy (IAGA) Division V, Working Group 8, who merge various geomagnetic field representations used to calculate the Earth's main magnetic field and its secular variation into one universal model. The resulting IGRF2000 formula relates the magnetic field to the dependent variables elevation (radius from Earth's center). latitude. and longitude. up to two decimal places. The formula itself defines a set of Gauss coefficients, $g_{n}$ and $h_{n}$, contained in a geomagnetic potential function in a series expansion truncated at $N=10$ (120 coefficients) (IAGA, 2000). The equation is as follows:

$$
V=a \sum_{n=1}^{N} \sum_{m=0}^{n}\left(\frac{a}{r}\right)^{n+1}\left(g_{n}^{m} \cos m \phi+h_{n}^{m} \sin m \phi\right) P_{n}^{m}(\cos \theta)
$$

where $g_{n}$ and $h_{n}$ are the Gauss coefficients, $a$ is the mean radius of Earth $(6371.4 \mathrm{~km}), r$ is the distance from the center of Earth (elevation in $\mathrm{km}$ ), $\phi$ is the decimal longitude east of 
the prime meridian, $\theta$ is the decimal colatitude ( $90^{\circ}$ minus the latitude), $V$ is the magnetic field (given in its three vector components and their sum), and $P_{n}(\cos \phi)$ are Schmidt quasi-normalized associated Legendre functions of degree $n$ and order $m(n \geq 1$ and $m \leq$ n) (IAGA, 2000). Output was determined through the use of a designed program, "IGRF2000," which utilizes the IGRF model equation. The output of the calculation also has a maximum of two decimal places.

To correct for diurnal variation of the magnetic field, the same IGRF2000 model was used, but the elevation, latitude, and longitude variables were kept constant by choosing arbitrary coordinates within Lavic Lake playa $(34.66 \mathrm{~N}, 116.36 \mathrm{~W}, 0.50 \mathrm{~km}$ in elevation). The variation of the magnetic field at these coordinates was plotted in terms of time, in calendar years, from July 7, 2000 to January 5, 2001, bounding the times of data collection (Fig. 21). This correction is meant to account for known solar activity, solar storms, magnetic storms, and any other variations in the magnetic field predicted by the IGRF2000 model. A linear fit to the graph defines a simple relationship between the magnetic field and time:

$$
F_{1}=-69.345 t+187,869
$$

where $t$ is time in decimal years and $F_{t}$ is the magnetic field in $n T$. Note that this is a negative correlation. 


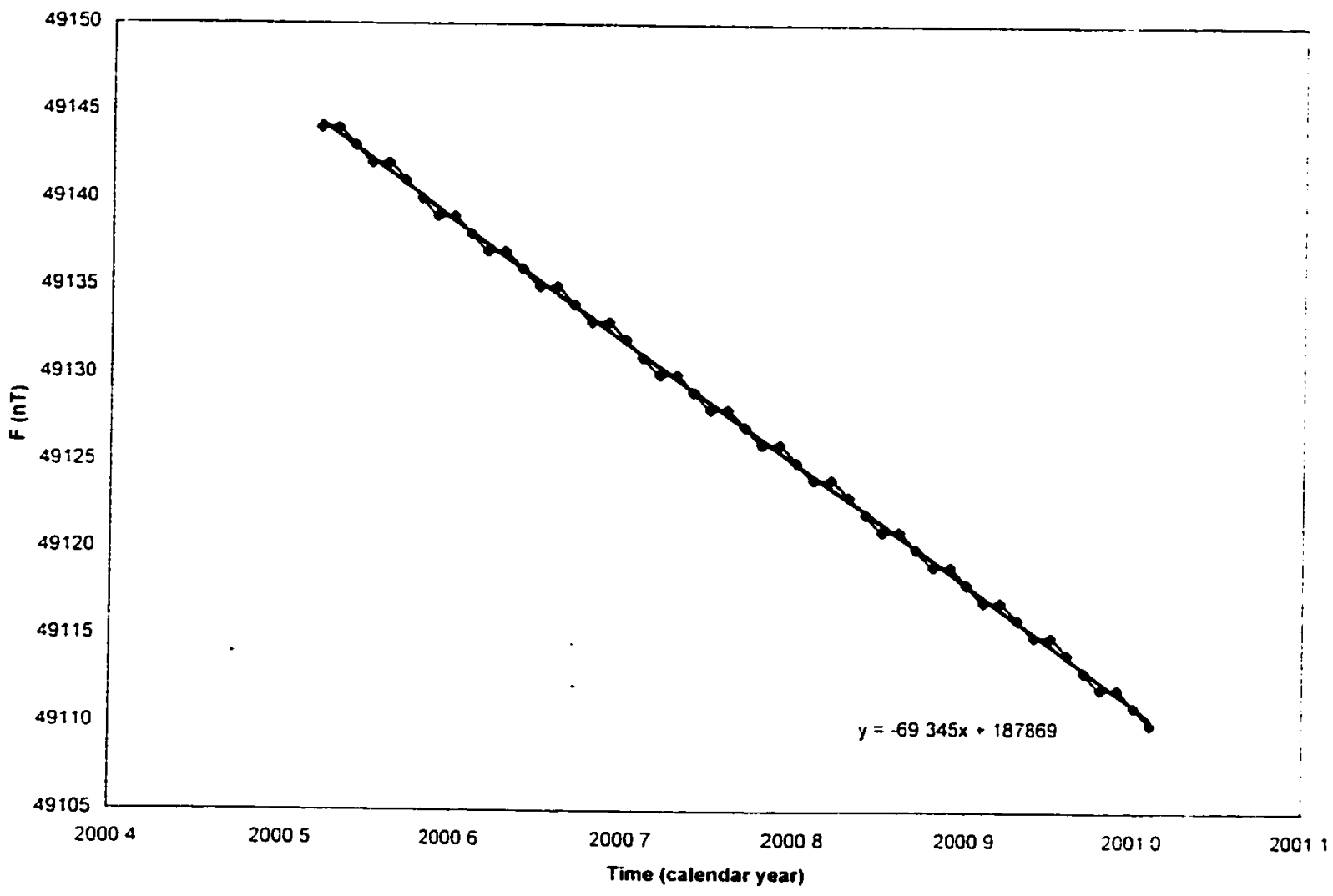

Figure 21. Plot of diurnal variation in the magnetic field at $34.66 \mathrm{~N}, 116.36 \mathrm{~W}(0.50 \mathrm{~km}$ in elevation) according to the IGRF2000 model. A linear fit to the data with its corresponding equation is indicated.

The relative change in magnetic field from the first measurement (49142.3865 nT) was calculated using the following equation:

$$
\Delta F=49142.3865-F_{1}
$$

where $\Delta \mathrm{F}$ is the relative change in the magnetic field.

To correct for the regional trend, I measured the magnetic anomaly on an aeromagnetic map of San Bernardino County (Fig. 22) over a distance of $6.5 \mathrm{~km}$ across Lavic Lake playa. The chosen distance displayed a relatively constant change in the magnetic anomaly, and the regional trend is reported as $65.8944 \mathrm{nT} / \mathrm{mi}$, dubbed the constant $R_{\text {con }}$ (Department of Conservation, Mines, and Geology, 1988). 


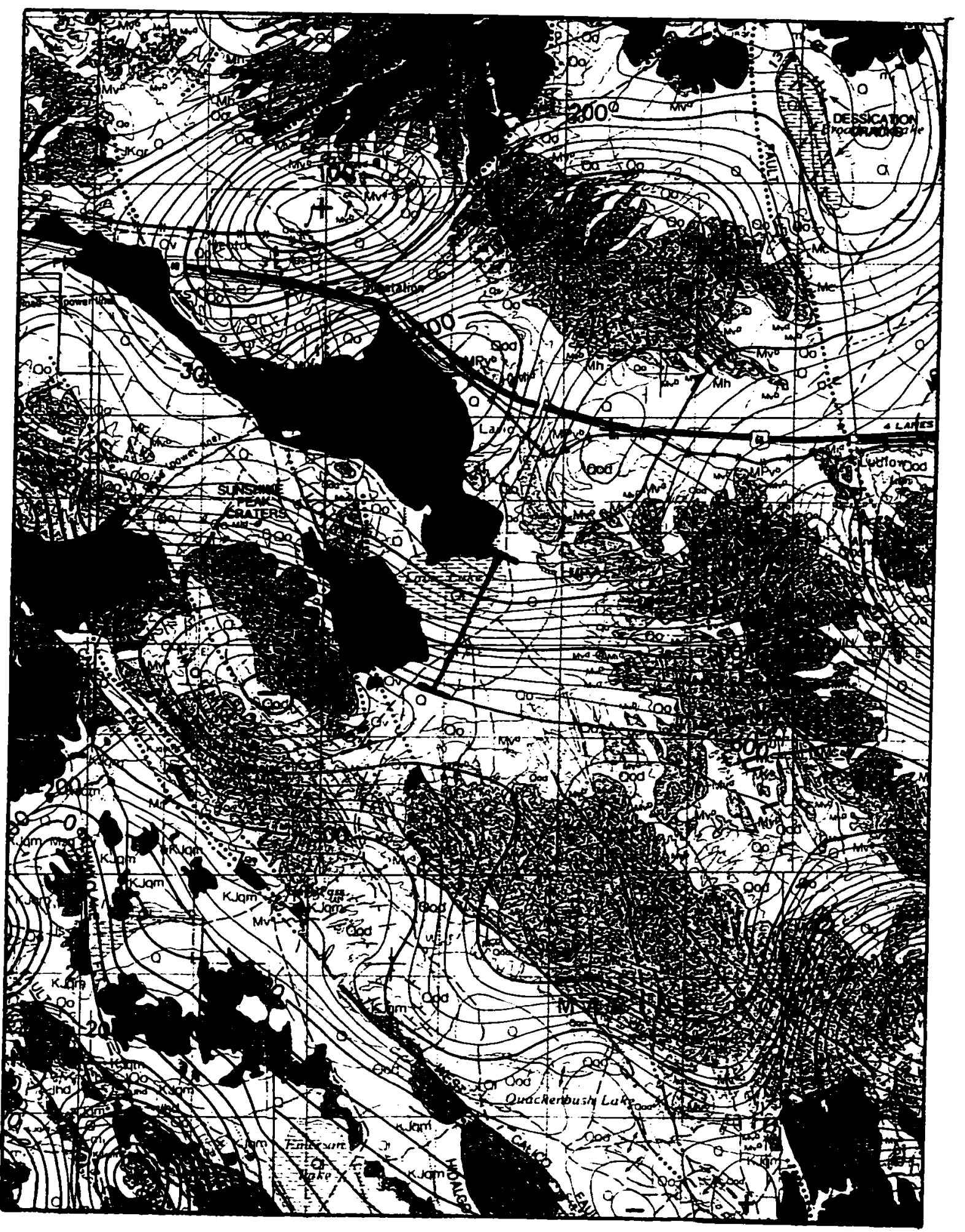

Figure 22. Portion of 1:250,000 scale aeromagnetic anomaly map of San Bernardino County. The regional trend in the magnetic anomaly was measured along the indicated line, trending northeast across Lavic Lake. Map modified from Department of Conservation, Mines, and Geology (1988). 
In the last step of this process, the residual magnetic anomaly was calculated in order to subtract all of the above effects in the magnetic field at any given point. This was found by the following equation:

$$
F_{a}=F-\Delta F-V-R_{c o n}
$$

where $F_{a}$ is the residual magnetic anomaly, and $F$ is the original reading taken from the magnetometer.

All calculated magnetic anomalies were imported into their respective survey locations using ArcView 3.2a software. The resulting data set was used to produce a contour map of the magnetic anomalies in the field area.

\section{Seismic Refraction Data}

Seismic refraction data were plotted on a graph of distance versus time, with the $\mathrm{x}$-axis displaying distance of the geophone from the source of the shot, and the $\mathrm{y}$-axis displaying the arrival time of the direct or refracted wave, in milliseconds. To maintain clarity, forward and reverse shots were not superimposed. On such a graph, a crossover distance (if any) was identified simply as an inflection point between two different slopes in the graph. If no crossover distance was found, the arrival time farthest from the shot was considered the crossover distance, and the following equation was used to calculate a minimum depth to basalt bedrock: 


$$
z=\left(\frac{1}{2}\right) \frac{\mathrm{V}_{1} \mathrm{~V}_{2}\left(T-\frac{x_{C}}{\mathrm{~V}_{2}}\right)}{\left[\left(\mathrm{V}_{2}\right)^{2}-\left(\mathrm{V}_{1}\right)^{2}\right]^{0.5}}
$$

where $z$ is the minimum depth to basalt bedrock (in $m$ ), $V_{1}$ is the seismic velocity of the overlying material (in $\mathrm{m} / \mathrm{s}), \mathrm{V}_{2}$ is the velocity of basalt bedrock $(3.87 \mathrm{~km} / \mathrm{s}$, or $3870 \mathrm{~m} / \mathrm{s}$ ) (assumed constant if no crossover was found), $x_{c}$ is the crossover distance from the shot (in $\mathrm{m}$ ), and $T$ (in units of seconds) is the y-intercept of the linear fit of the graph showing the greatest seismic velocity. Seismic velocities of the graph segments were determined by computing the reciprocal of the respective slope, and are reported in units of $\mathrm{ft} / \mathrm{ms}$, $\mathrm{ft} / \mathrm{s}$, and $\mathrm{km} / \mathrm{s}$. The value $3.87 \mathrm{~km} / \mathrm{s}$ chosen for basalt bedrock is the average weighted velocity of seismic waves calculated from all instances where basalt bedrock was encountered during the surveys.

Shots yielded results of variable quality, probably due to variable field conditions (e.g., variations in thickness of lake sediment) and equipment problems (e.g., electrical malfunctions in some geophones). In some shots, scatter of the data made it difficult to estimate accurate seismic velocities. In other instances, geophones farther from the shot yielded times that were earlier than a geophone closer to the shot. Due to these inconsistencies, shots were given a qualitative grade in order to describe the overall quality of the shot data and the ability of the shot data to yield accurate results. 
A: The data from the shot show minimal scatter with all points on or very close to a best-fit line.

B: The data show some scatter with some points not on or very close to a best-fit line.

C: The data show significant scatter from a best-fit line. Best-fit lines applied to these data are assumed to be only somewhat accurate, although usable.

D: The data show obvious, pronounced scattering, with poor correspondence to a best-fit line. Only a few data points in the shot can be used.

F: The data are so scattered that best-fit lines are inapplicable. Results appear to contradict physical laws, and are not usable.

This grading system was employed to calculate the weighted-average seismic velocity of basalt bedrock in the field area $(3.87 \mathrm{~km} / \mathrm{s})$. In $A$ shots, the velocity found was used three times. In B shots, the velocity found was used two times. In $C$ shots, the velocity found was used one time. D and F shots were not considered in the calculation due to their low confidence.

\section{DGPS/GIS Data}

\section{Differential Corrections}

Corrections used files from a GPS base station operated by the United States Forest Service, Sequoia National Forest, Porterville, California, located about $400 \mathrm{~km}$ northwest of Lavic Lake. This was the nearest GPS base station available. Trimble Pathfinder Office version 2.51 software was used to perform the differential correction.

\section{ArcView 3.2a Data Manipulation}

All spatial data files collected in the field were exported into ArcView 3.2a to 
shapefile format, the standard vector-based spatial data format used by ArcView GIS. The exporting process successfully transferred most data points with the respective date (standard format $\mathrm{mm} / \mathrm{dd} / \mathrm{yy}$ ), time (Pacific Standard or Daylight time), latitude and longitude (in decimal degrees), and elevation (in meters) at which they were taken. The data points (approximately 20,000 total) were combined into one large two-dimensional shapefile (planar map view) and one large three-dimensional shapefile for display as a digital elevation model (DEM). Elevation points that did not match field observations were removed from the DEM.

Separate shapefiles contained data points associated with either geologic contacts, the Hector Mine rupture, specific magnetic or seismic surveys, or other observed features. The resulting two-dimensional shapefiles were used to create maps of the area (Figs. 16, 17, 20). A new field was created in the magnetic survey shapefiles for the magnetic anomaly of each data point. The resulting data set was then contoured to display relatively high and low values of the magnetic anomaly on mapped features on the playa (Fig. 23). 

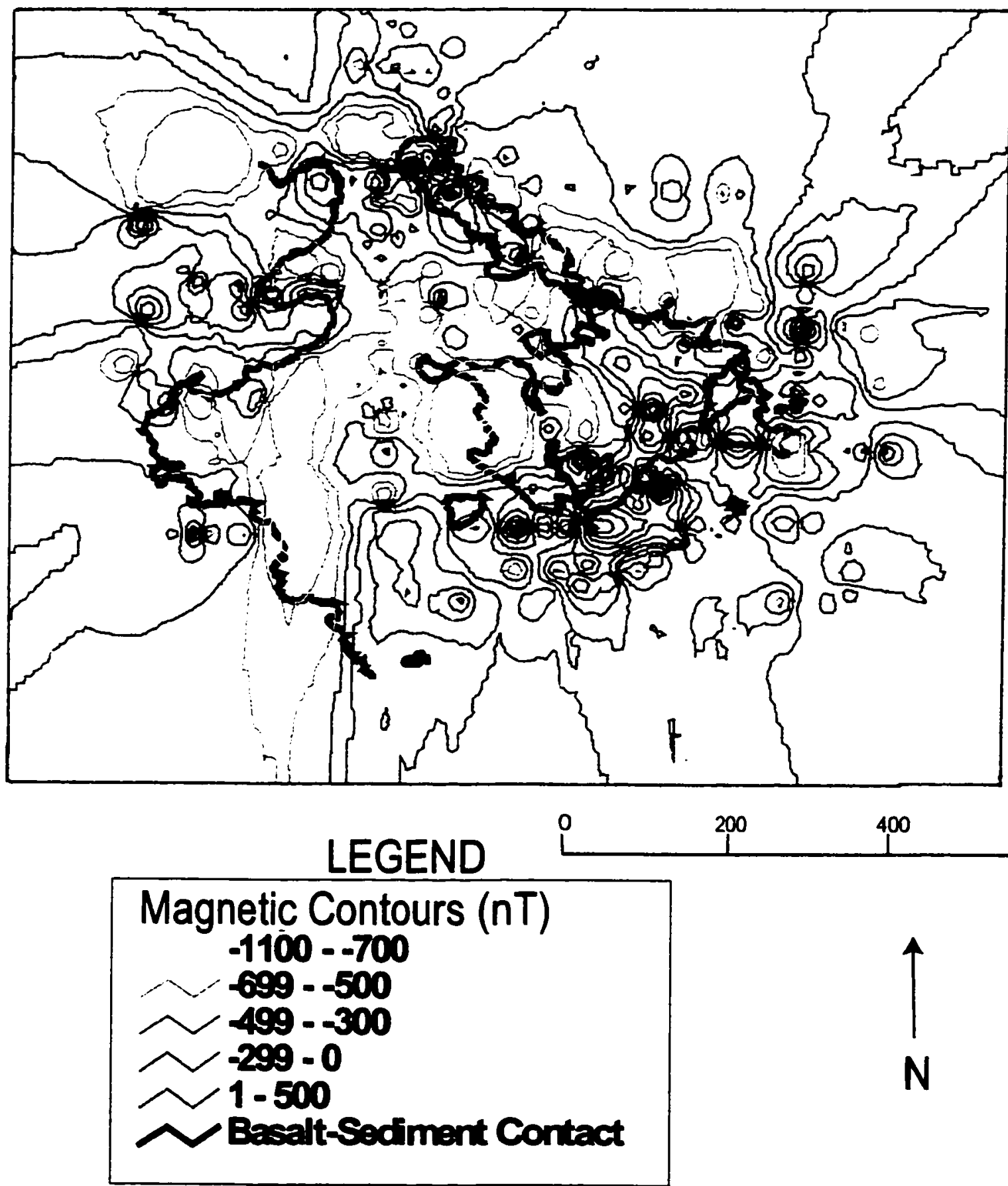

Figure 23. Magnetic anomaly contour map of the field area. The base contour is $-1200 \mathrm{nT}$, and the contour interval is $100 \mathrm{nT}$. This figure was generated by rover files imported into ArcView 3.2a. 
RESULTS

\section{Magnetic Anomalies and Modeling}

The calculated residual magnetic anomalies give a good representation of the magnetic character of the field area. The entire data set is presented in Table Al of the appendix, and summaries of important points are presented below.

\section{Magnetic Anomaly Contour Mapping}

The contour map generated by the calculated magnetic anomalies is shown in

Figure 23. In general, low magnetic anomalies exist predominantly in areas underlain by clay and sand, including (1) the area between the hand and the Lava Bed Mountains, (2) the northeast side of the "finger" (the northern elongate section of the hand), (3) at the very tip of the finger, and (4) west of the finger tip. High magnetic anomalies are found on the south side of the hand, where basalt is exposed, and southwest of the hand.

\section{Trends in the Linear Magnetic Profiles Across the Hand}

Magnetic anomalies calculated from data along the 12 linear survey lines are plotted on graphs showing horizontal distance versus the magnetic anomaly (in $\mathrm{nT}$ ). Magnetometer line 1 runs along the southern base of the hand, crossing the Hector Mine rupture (Fig. 16). From $0 \mathrm{ft}$., at the edge of the Lava Bed Mountains, to $300 \mathrm{ft}$., the magnetic anomaly remains relatively low, never exceeding $-475 \mathrm{nT}$ (Fig. 24). At $500 \mathrm{ft}$., the magnetic anomaly increases to almost $15 \mathrm{nT}$, and then steadily decreases to about $580 \mathrm{nT}$ near $750 \mathrm{ft}$. The local minimum from $700 \mathrm{ft}$. to $900 \mathrm{ft}$. coincides with the proximity of a large basalt "island" at the southwest edge of the hand. A large spike 


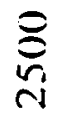

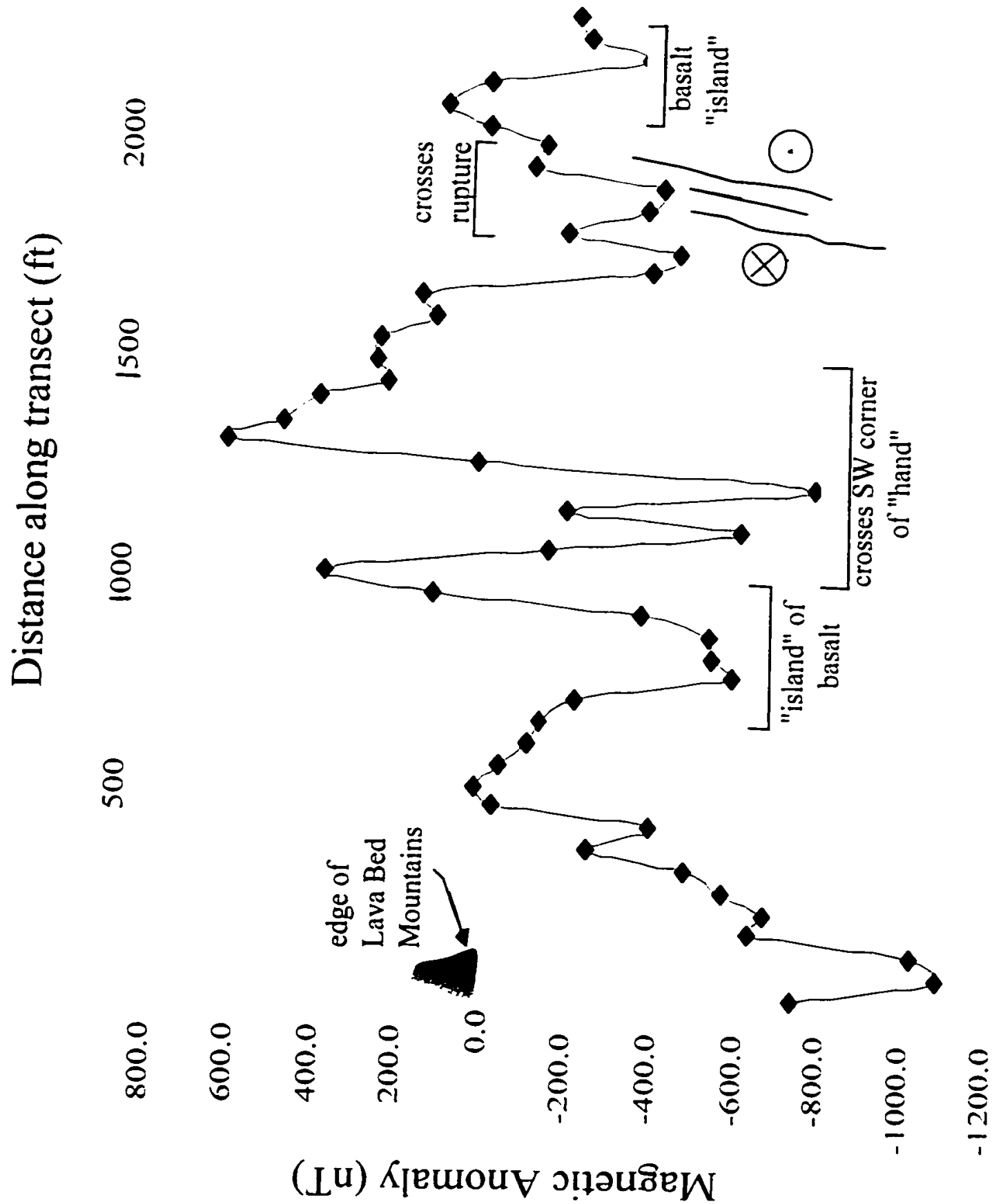

Figure 24. Magnetic anomaly plot of magnetometer line I. 
in the magnetic anomaly at $1000 \mathrm{ft}$. reaches $385 \mathrm{nT}$, but over the next $100 \mathrm{ft}$. values drop to $-600 \mathrm{nT}$. From 1310 to $1650 \mathrm{ft}$. the magnetic anomaly increases dramatically to reach dominantly positive values, with a maximum of $622 \mathrm{nT}$. These positive values coincide with the position of the basalt rocks found on the southwest comer of the hand. For the remainder of the line, clearing the southern base of the hand, the anomaly remains relatively low except for a small increase to $114 \mathrm{nT}$ at $2100 \mathrm{ft}$. The line crosses the Hector Mine rupture from $1900 \mathrm{ft}$. to $2100 \mathrm{ft}$, approximately coinciding with a local minimum.

Magnetometer line 3 parallels the trend of line 1, surveying the playa sediment to the northeast and southwest of the hand (Fig. 16). The entire line displays predominantly negative magnetic anomalies, except near $650 \mathrm{ft}$., where the anomaly reaches as high as $425 \mathrm{nT}$ (Fig. 25). The highest value of $425 \mathrm{nT}$ is found directly in the center of the mapped Hector Mine rupture. The positive value of approximately $125 \mathrm{nT}$ at $50 \mathrm{ft}$. coincides with a section of basalt bedrock at the base of the Lava Bed Mountains. Negative anomalies from $1000 \mathrm{ft}$. to $1250 \mathrm{ft}$. coincide with small mapped cracks in the playa sediment believed to be a result of the 1999 surface rupture.

Magnetometer line 4 began in playa sediment on the west side of the hand and crossed the bottom section of the finger (Fig. 16). A marked increase in the magnetic anomaly from $150 \mathrm{ft}$. to $300 \mathrm{ft}$. coincides with basalt bedrock at the base of the Lava Bed Mountains (Fig. 26). One positive magnetic anomaly (305 nT) at $1350 \mathrm{ft}$. coincides with basalt that composes the finger. Local minima on either side of this maximum mark the position where the survey crosses the Hector Mine rupture and "overthrust" playa 
焉

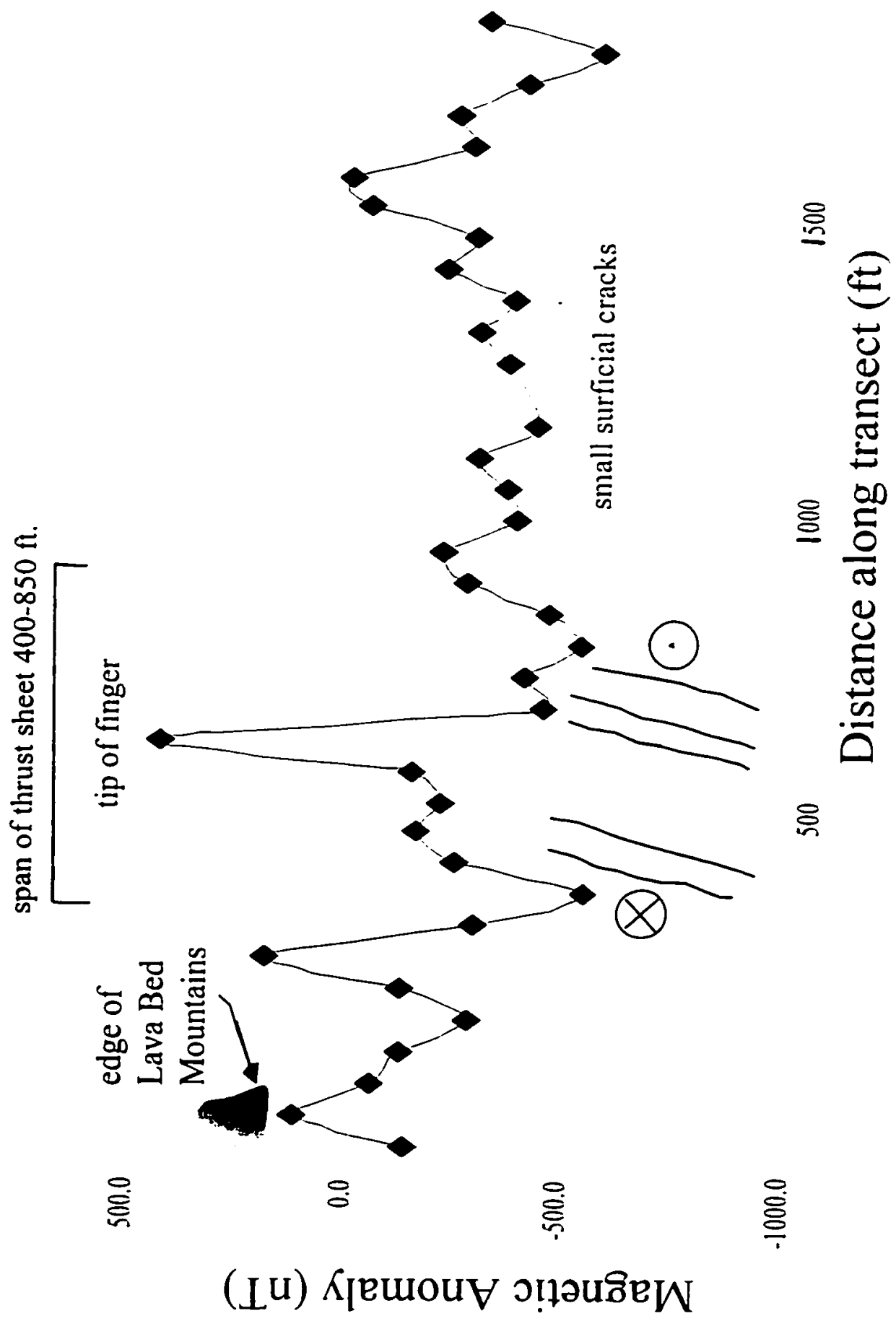

Figure 25. Magnetic anomaly plot of magnetometer line 3. 


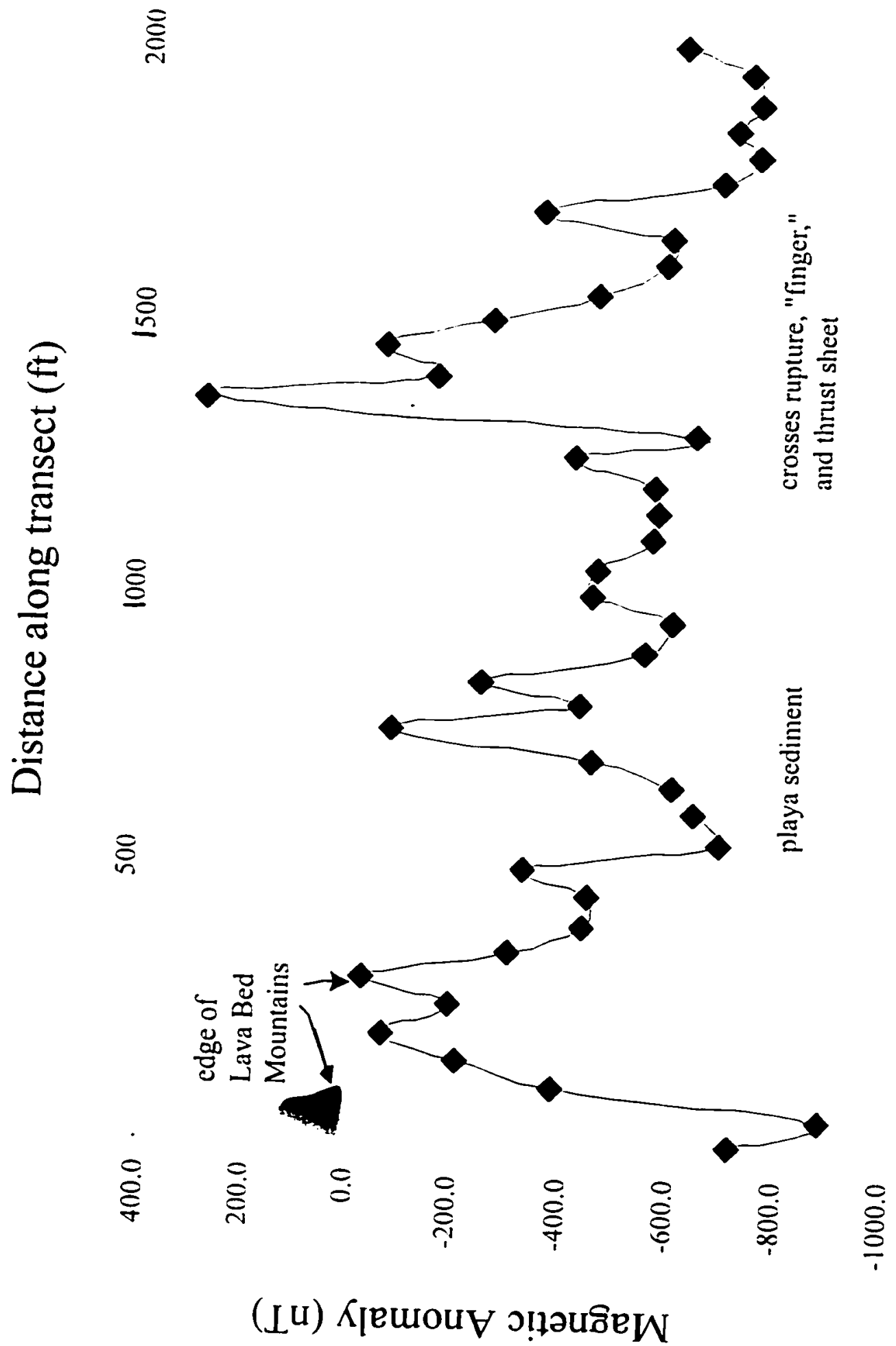

Figure 26. Magnetic anomaly plot of magnetometer line 4. 
deposits (clay).

On magnetometer line 29, which extends $3000 \mathrm{ft}$. from the base of the Lava Bed Mountains eastward across the hand (Fig. 16), the magnetic anomalies remain negative throughout the playa sediment on the west side of the hand (Fig. 27). Erratic positive and negative values are evident east of $1950 \mathrm{ft}$., where the line begins to cross the basalt that composes the main part of the hand. However, the magnetic anomaly reaches a maximum value of only $350 \mathrm{nT}$ at $2350 \mathrm{ft}$. Negative values from $2100 \mathrm{ft}$. to $2300 \mathrm{ft}$. were obtained where the survey line crosses the Hector Mine rupture.

\section{Trends in the Linear Magnetic Profiles in Playa Sediment}

Magnetometer line 5, which runs perpendicular to lines 1-4, extends entirely within playa sediment on the west side of the hand (Fig. 16). The anomalies shown in the plot are all negative except for one positive value of $16.4 \mathrm{nT}$ at $100 \mathrm{ft}$. (Fig. 28). This reading was taken approximately between the basalt body at the base of the Lava Bed Mountains and the tip of the finger. Magnetometer line 30, a continuation of line 5 that crosses the south end of the hand (Fig. 16), also has predominantly negative anomalies, with two positive values that do not exceed 25 nT (Fig. 29).

In contrast, Line 34 , on the east side of the hand, displays some markedly positive magnetic anomalies. At $250 \mathrm{ft}$, the magnetic anomaly reaches $191 \mathrm{nT}$, decreases sharply in $50 \mathrm{ft}$, and then increases again to $269 \mathrm{nT}$ at $350 \mathrm{ft}$. (Fig. 30). The highest positive anomaly is found at $700 \mathrm{ft}$., with a value of $652 \mathrm{nT}$. No mapped features on the playa coincide with the position of these local maxima. 


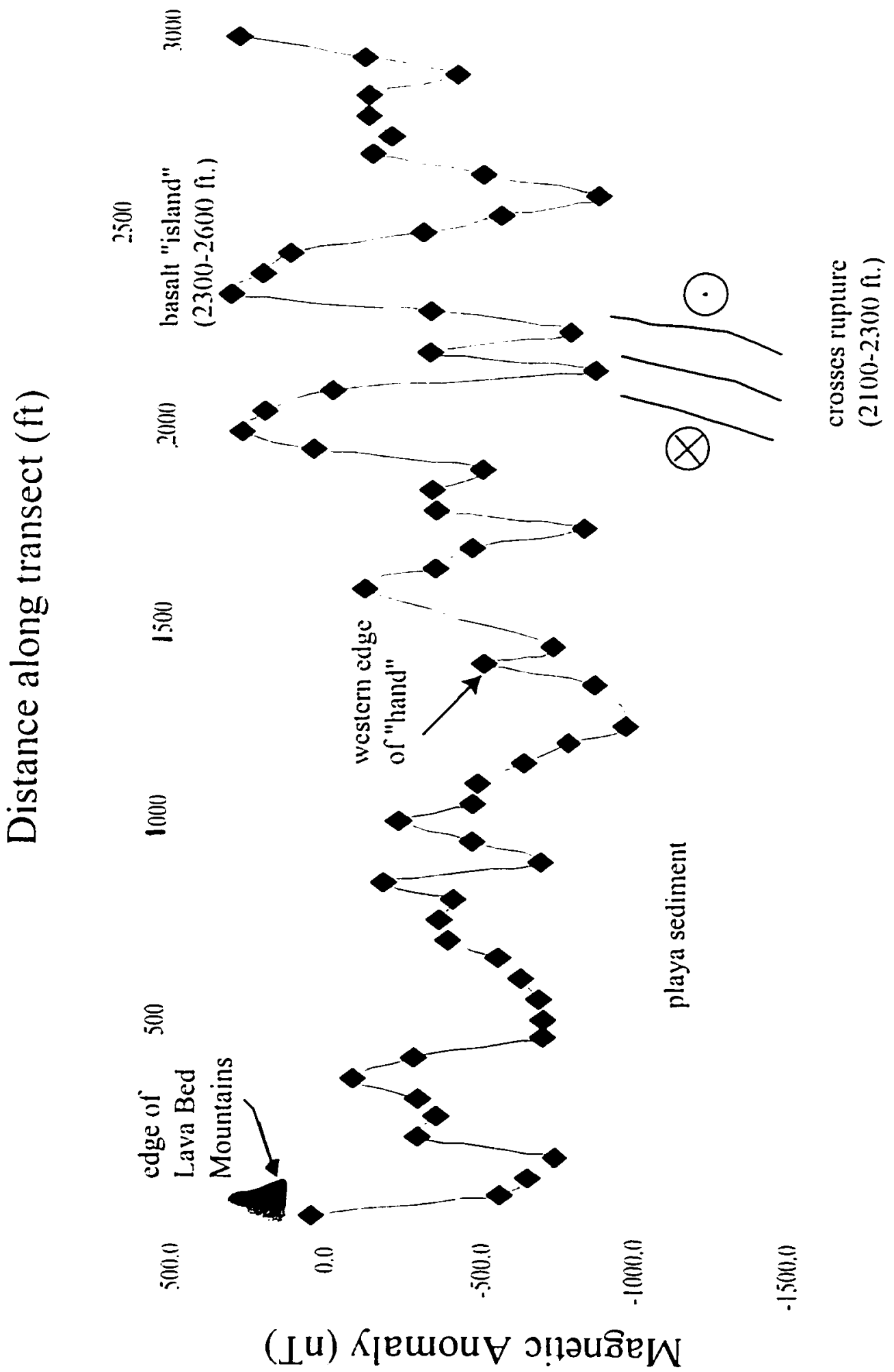

Figure 27. Magnetic anomaly plot of magnetometer lia:e 29. 


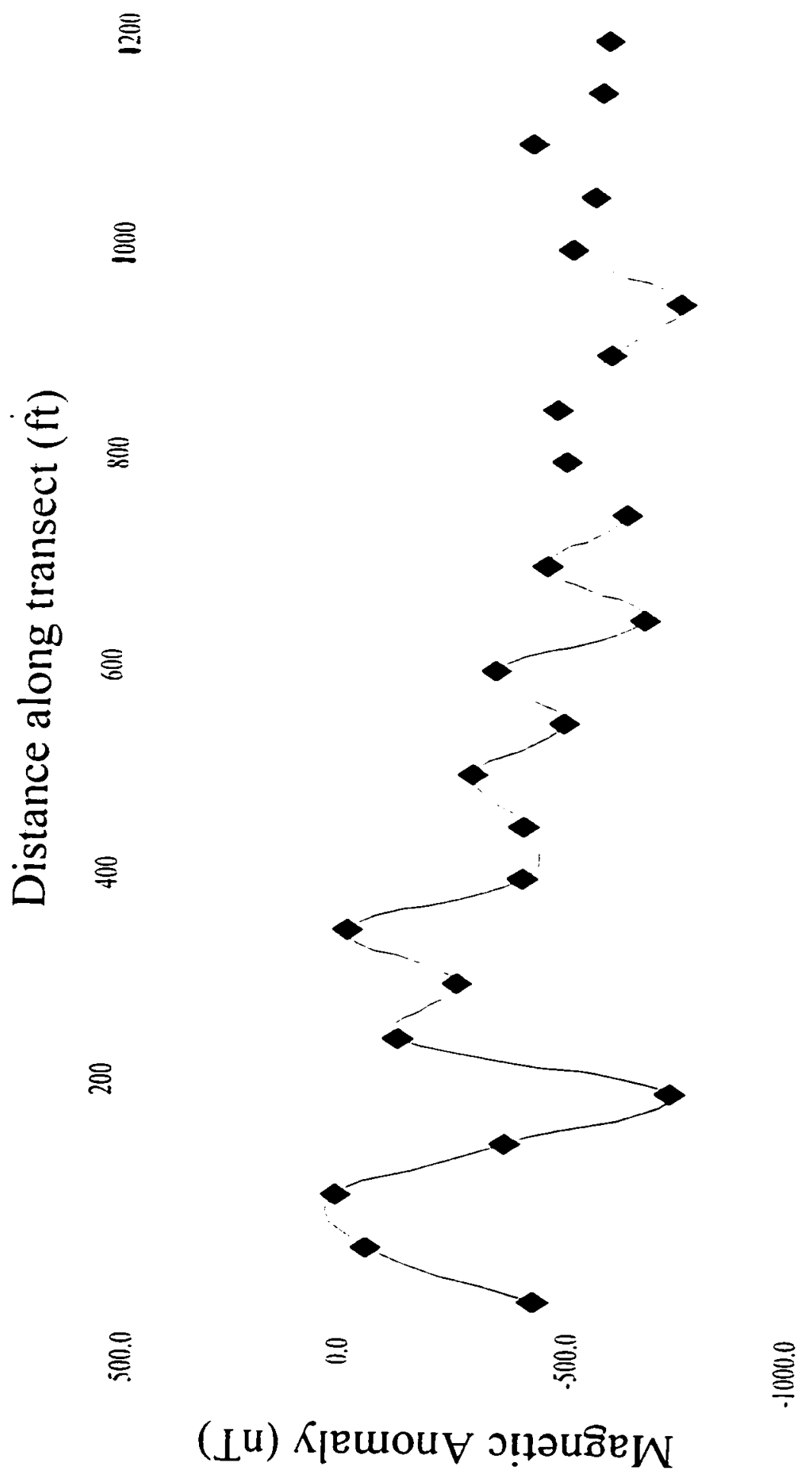

Figure 28. Magnetic anomaly plot of magnetometer line 5. 


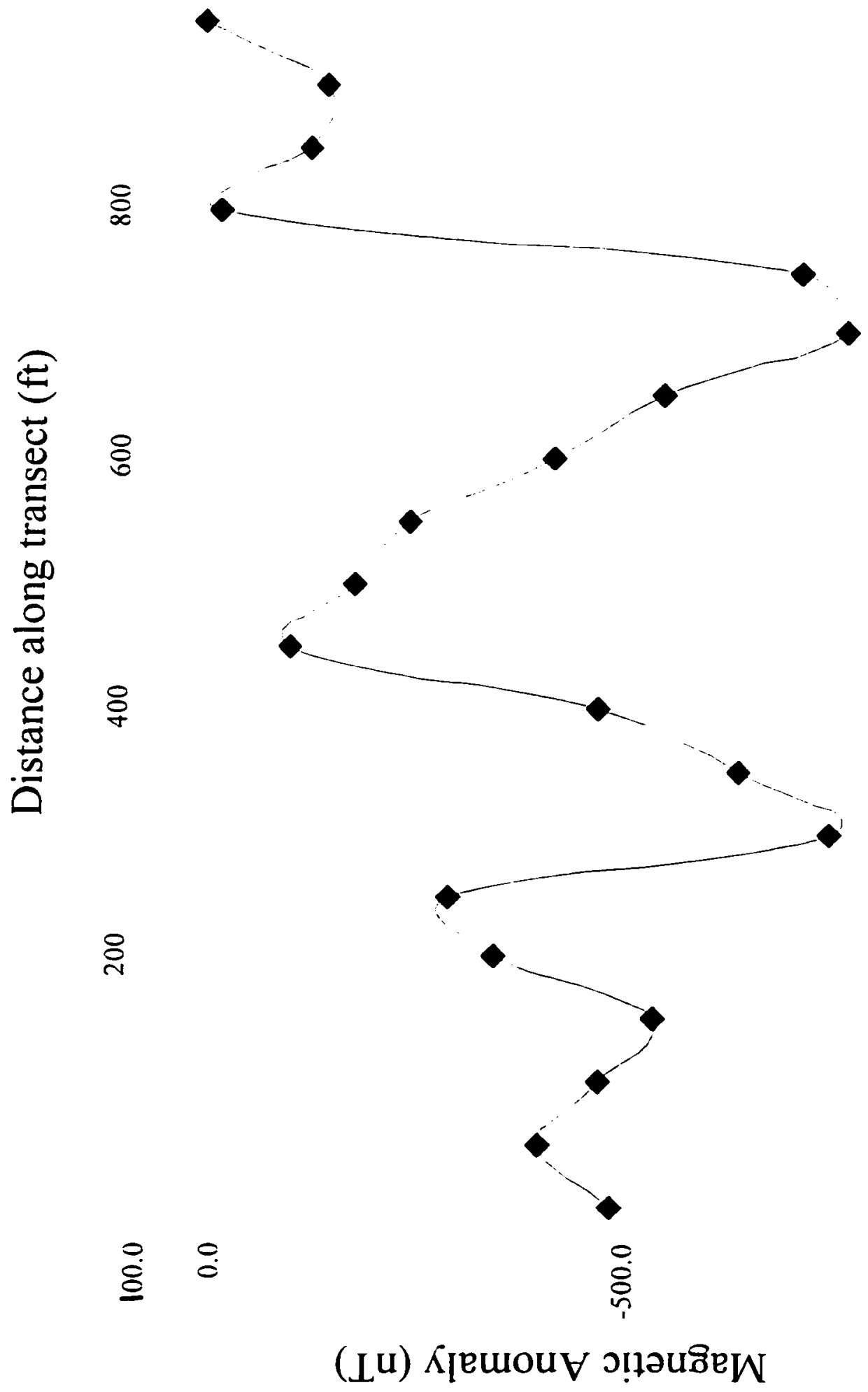

Figure 29. Magnetic anomaly plot of magnetometer line 30. 


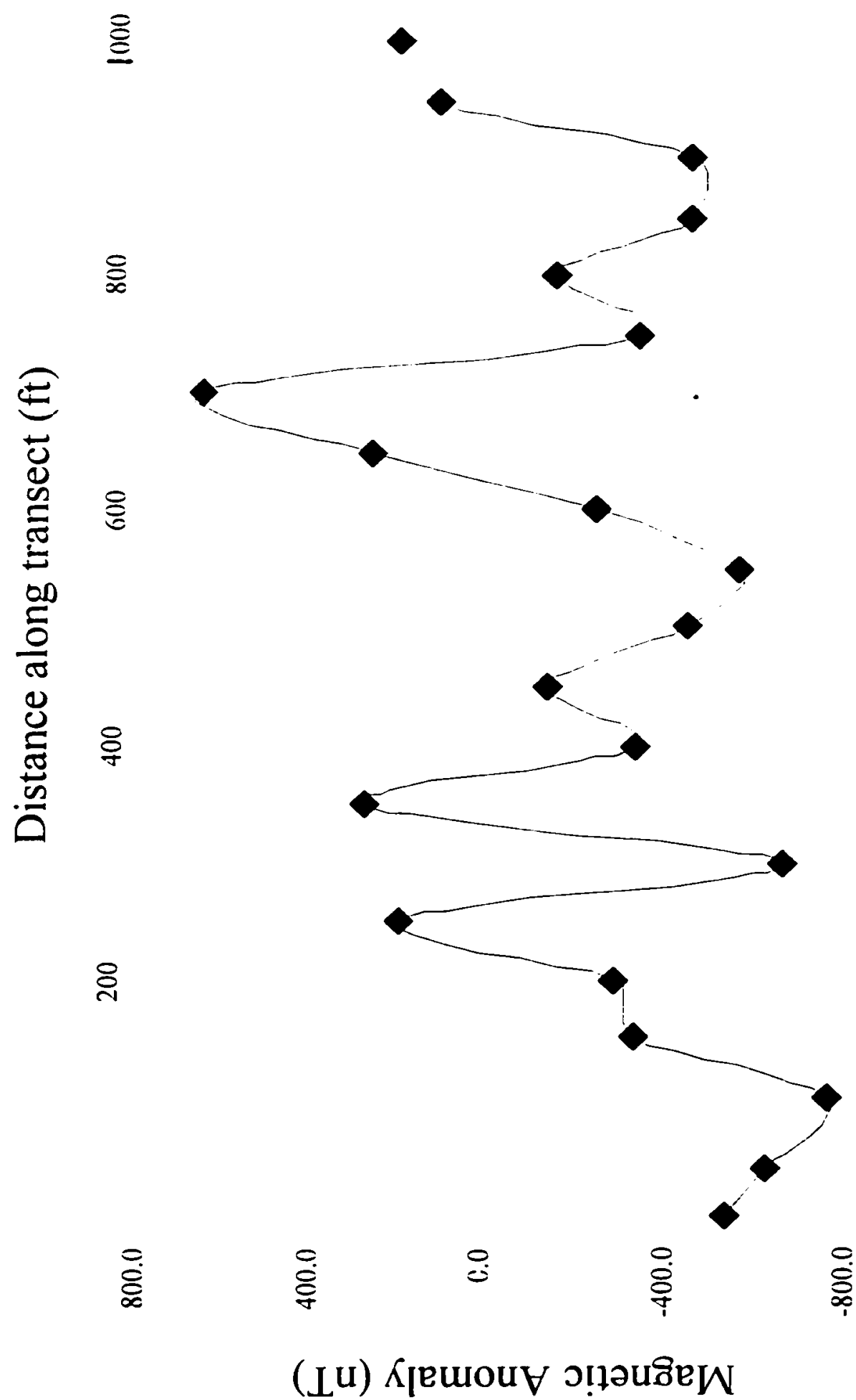

Figure 30. Magnetic anomaly plut of magnetometer line 34. 


\section{Trends in the Linear Magnetic Profiles Across the Hector Mine Rupture}

As discussed in a previous section, magnetometer lines 1, 3, and 4 all cross the Hector Mine rupture. On lines 1 and 4 , the rupture is coincident with marked negative anomalies (Figs. 24, 26). However, on line 3, high magnetic anomalies are found at the position of the rupture, while negative anomalies coincide with 'small mapped cracks believed to be the result of the earthquake (Fig. 25).

Magnetometer line 23 (Fig. 16) crosses the Hector Mine rupture from $240 \mathrm{ft}$. to $360 \mathrm{ft}$., although the rupture was not mapped by GPS in this area. The projected location of the rupture on line 23 is marked by a obvious decrease in the magnetic anomaly (Fig. 31). Positive values near the east end of the line do not coincide with any mapped features besides playa sediment.

Magnetometer line 24 (Fig. 16) crosses the Hector Mine rupture from $300 \mathrm{ft}$. to $500 \mathrm{ft}$., although the rupture was not mapped in this area. Magnetometer line 25 (Fig. 16) also crossed the rupture at $300 \mathrm{ft}$. to $500 \mathrm{ft}$, where the rupture makes a major left step. This left step is the location of two trenches excavated by the USGS in April 2000 (M. Rymer, personal communication). The projected location of the rupture on line 24 is marked by a maximum bounded by two minima in the magnetic anomaly (Fig. 32). Line 25 likewise shows a local maximum with bounding minima where it crosses the rupture (Fig. 33). Line 25 is about $100 \mathrm{~m}$ northwest of line 24 (Fig. 16).

Magnetometer line 26 (Fig. 16) crosses another major left-stepping fault jog in the Hector Mine rupture from $400 \mathrm{ft}$. to $550 \mathrm{ft}$., although the rupture was not mapped in this area. From the western end of the line, the magnetic anomaly steadily increases to almost 


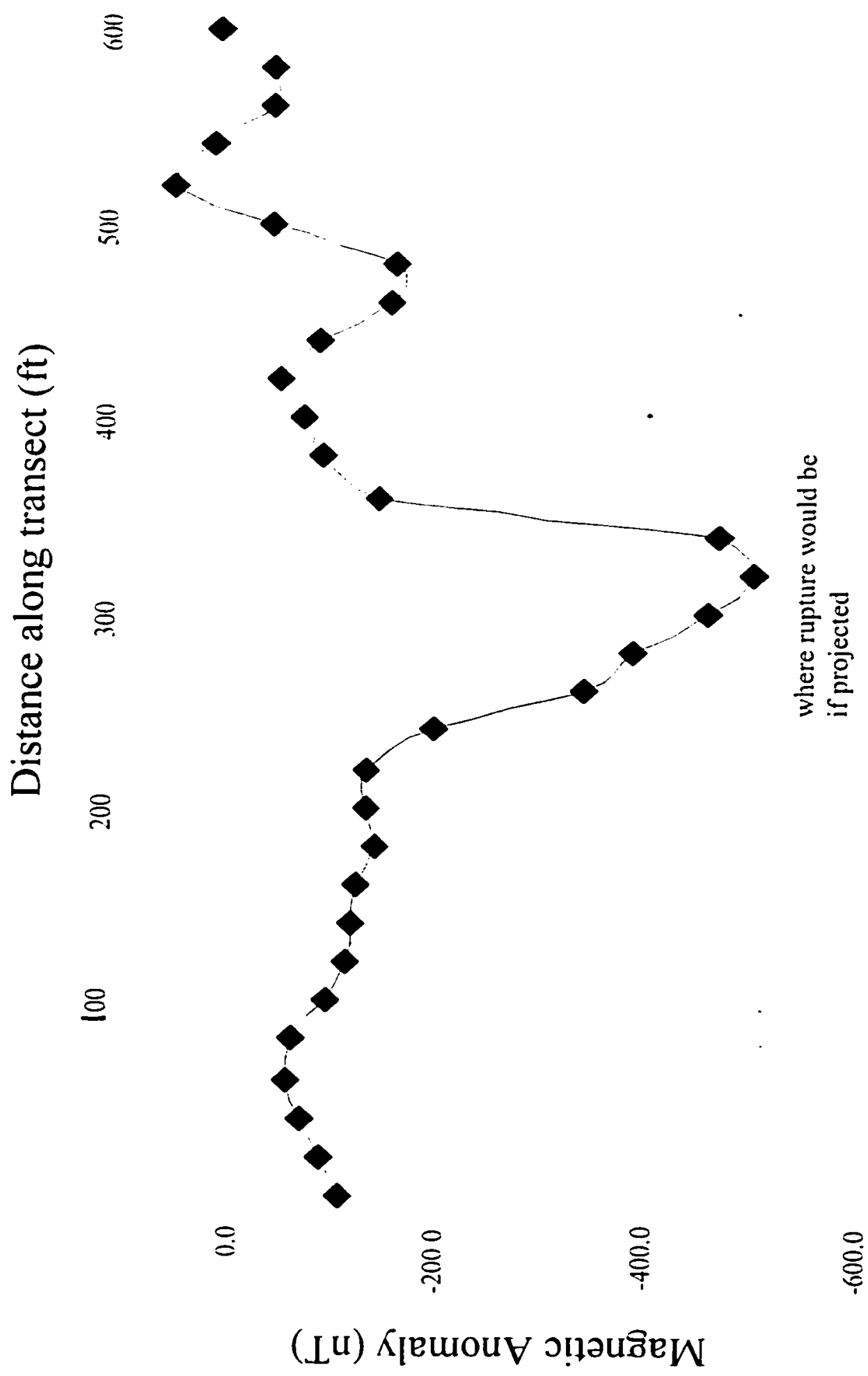

Figure 31. Magnetic anomaly plot of magnetometer line 23. 


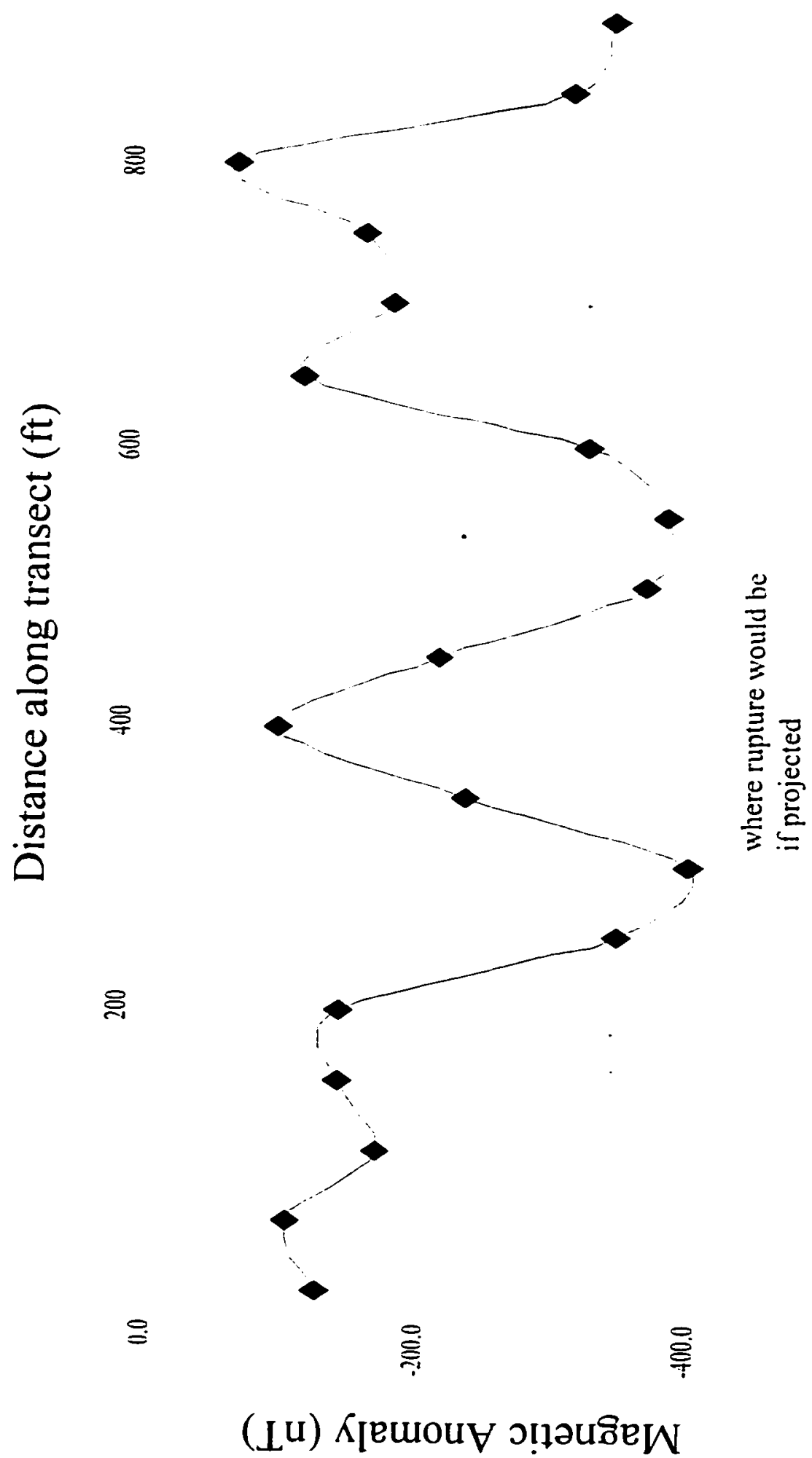

Figure 32. Magnetic anomaly plot of magnetometer line 24. 


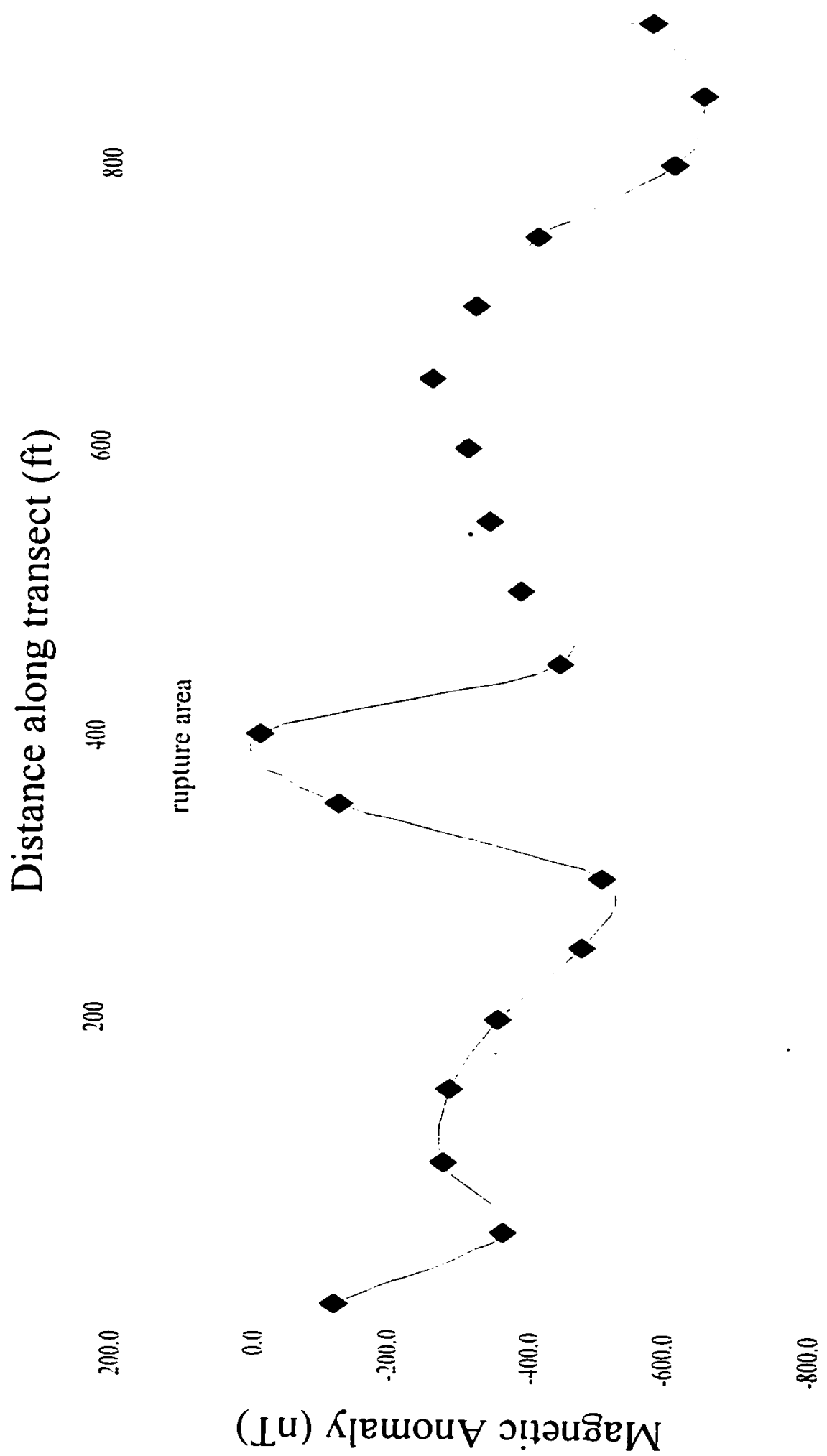

Figure 33. Magnetic anomaly plot of magnetometer line 25. 
$150 \mathrm{nT}$, and then shows a sharp decrease where the fault jog is encountered (Fig. 34). The magnetic anomaly remains negative along the eastern half of the survey line.

On magnetometer line 29 , as discussed in the previous section, negative values from $2100 \mathrm{ft}$. to $2300 \mathrm{ft}$. are found where the survey crossed the Hector Mine rupture (Fig. 27). These local minima in the magnetic anomaly are bounded by relatively positive maxima.

\section{Trends in Other Linear Magnetic Profiles}

Magnetometer line 2, colinear with line 1, lies wholly within the Lava Bed Mountains. This line placement was chosen to evaluate magnetic anomalies in areas of known basalt bedrock. As seen from Figure 35, the magnetic anomaly can be highly irregular even on basalt bedrock. Values of the anomaly range from $-530 \mathrm{nT}$ to almost $260 \mathrm{nT}$.

\section{Seismic Refraction}

\section{Seismic Lines West of the Hand}

Seismic lines $1,2,6,12,16-20,27$, and 28 transect the playa sediment between the hand and the Lava Bed Mountains (Fig. 17). Data collected from those lines address the subsurface structure and depth of the basalt in that region. All data collected from shots and calculations are tabulated in Appendix 2.

Line 1, southwest of the hand, yielded refraction data with no crossovers, indicating that basalt was not encountered within the penetration depth of the survey. The data are summarized in Table 1, and individual shots are presented in Tables A2-A9. 


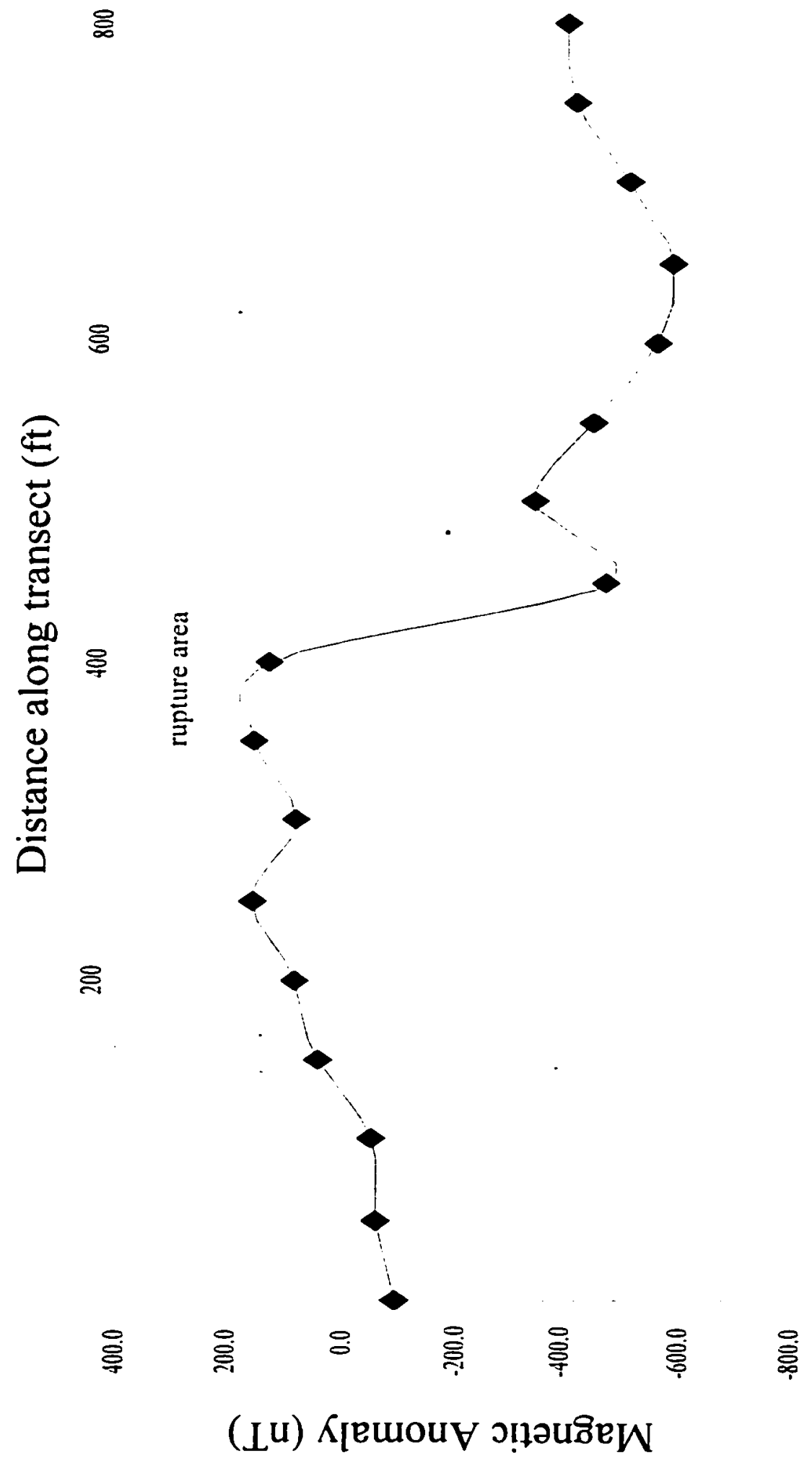

Figure 34. Magnetic anomaly plot of magnetometer line 26. 
응

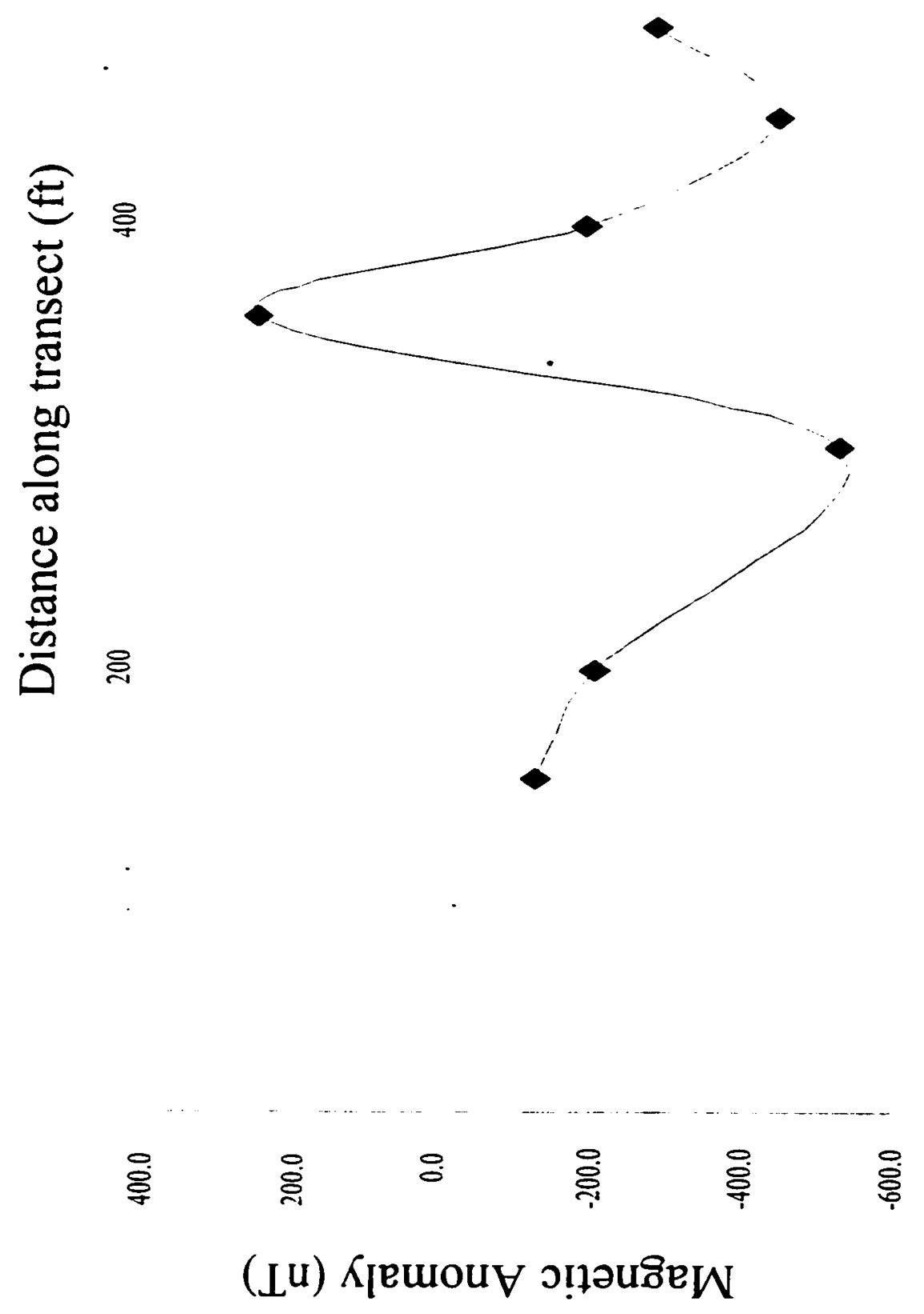

Figure 35. Magnetic anomaly plot of magnetometer line 2. 
Because no crossovers were seen, sections of the graph with the highest seismic velocities were chosen for the subsequent velocity and minimum depth calculations. Thus, $V_{1}$ are maxima. These data show that basalt bedrock could lie anywhere from 17 $\mathrm{m}$ to almost $80 \mathrm{~m}$ below the playa sediment in this region of the field area.

TABLE 1. SEISMIC LINE 1, SHOTS 1-8

\begin{tabular}{lllllll}
\hline Shot & $V_{1}(\mathrm{~km} / \mathrm{s})$ & $V_{2}(\mathrm{~km} / \mathrm{s})$ & Crossover? & $\begin{array}{l}\text { If Yes, Depth } \\
\text { to Basalt } \\
\text { Refractor }(\mathrm{m})\end{array}$ & $\begin{array}{l}\text { If No, } \\
\text { Minimum } \\
\text { Depth to } \\
\text { Refractor }(\mathrm{m})\end{array}$ & $\begin{array}{l}\text { Grade } \\
\text { of } \\
\text { Shot }\end{array}$ \\
\hline 1 & 1.70 & 3.87 & no & & 44.54 & B \\
2 & 1.65 & 3.87 & no & & 55.35 & B \\
3 & 1.91 & 3.87 & no & & 47.35 & B \\
4 & 1.69 & 3.87 & no & & 55.50 & B \\
5 & 0.53 & 3.87 & no & 19.94 & C \\
6 & 2.12 & 3.87 & no & 78.11 & C \\
7 & 0.67 & 3.87 & no & 17.45 & C \\
8 & 1.25 & 3.87 & no & & 47.15 & B \\
\hline
\end{tabular}

Line 2, on the edge of the Lava Bed Mountains near the northern tip of the hand, did show a crossover, but only on the first (forward) shot (Table 2, Tables A10-A13). The reverse shot (shot 2, Table Al1) did not yield reliable data to determine a reciprocal time. No other crossovers were seen with subsequent shots.

Line 6, which crosses the 1999 Hector Mine rupture near the west end of the finger, also shows no crossovers (Table 3, Tables A14-A15). Only minimum depths to bedrock could be calculated. 
TABLE 2. SEISMIC LINE 2, SHOTS $1-4$

\begin{tabular}{lllllll}
\hline Shot & $V_{1}(\mathrm{~km} / \mathrm{s})$ & $\mathrm{V}_{2}(\mathrm{~km} / \mathrm{s})$ & Crossover? & $\begin{array}{l}\text { If Yes, Depth } \\
\text { to Basalt } \\
\text { Refractor }(\mathrm{m})\end{array}$ & $\begin{array}{l}\text { If No, } \\
\text { Minimum } \\
\text { Depth to } \\
\text { Refractor }(\mathrm{m})\end{array}$ & $\begin{array}{l}\text { Grade } \\
\text { of } \\
\text { Shot }\end{array}$ \\
\hline 1 & 0.39 & 4.23 & yes & 3.97 & & $\mathrm{~A}$ \\
2 & 0.74 & 3.87 & no & & 32.81 & $\mathrm{C}$ \\
3 & 0.88 & 3.87 & no & & 14.73 & $\mathrm{~A}$ \\
4 & 0.61 & 3.87 & no & & 36.62 & $\mathrm{~A}$ \\
\hline
\end{tabular}

TABLE 3. SEISMIC LINE 6, SHOTS 1 AND 2

\begin{tabular}{|c|c|c|c|c|c|c|}
\hline Shot & $V_{1}(\mathrm{~km} / \mathrm{s})$ & $V_{2}(\mathrm{~km} / \mathrm{s})$ & Crossover? & $\begin{array}{l}\text { If Yes, Depth } \\
\text { to Basalt } \\
\text { Refractor (m) }\end{array}$ & $\begin{array}{l}\text { If No, } \\
\text { Minimum } \\
\text { Depth to } \\
\text { Refractor (m) }\end{array}$ & $\begin{array}{l}\text { Grade } \\
\text { of } \\
\text { Shot }\end{array}$ \\
\hline I & 0.34 & 3.87 & no & & 13.60 & $A$ \\
\hline 2 & 0.41 & 3.87 & no & & 14.87 & A \\
\hline
\end{tabular}

Lines 12, 17, and 18 extend eastward from the finger into playa sediment between the hand and the Lava Bed Mountains (Fig. 17). The data from these lines are summarized in Tables 4-6, and individual shots are presented in Tables A35-A62, A79A82, and A83-A100. Some crossovers into basalt bedrock were identified, but basalt bedrock could not be found with most shots. 
TABLE 4. SEISMIC LINE 12, SHOTS 1-29

\begin{tabular}{|c|c|c|c|c|c|c|}
\hline Shot & $\mathrm{V}_{\mathrm{l}}(\mathrm{km} / \mathrm{s})$ & $V_{2}(\mathrm{~km} / \mathrm{s})$ & Crossover? & $\begin{array}{l}\text { If Yes, Depth } \\
\text { to Basalt } \\
\text { Refractor (m) }\end{array}$ & $\begin{array}{l}\text { If No, } \\
\text { Minimum } \\
\text { Depth to } \\
\text { Refractor }(\mathrm{m}) \\
\end{array}$ & $\begin{array}{l}\text { Grade } \\
\text { of } \\
\text { Shot }\end{array}$ \\
\hline 1 & 1.27 & 3.87 & no & & 40.83 & $\mathrm{~A}$ \\
\hline 2 & 0.56 & 3.87 & no & & 14.79 & A \\
\hline 3 & 0.84 & 3.87 & no & & 18.54 & A \\
\hline 4 & 0.84 & 3.87 & no & & 23.50 & B \\
\hline 5 & 1.24 & 3.87 & no & & 29.75 & A \\
\hline 6 & 3.05 & 3.87 & no & & 170.11 & B \\
\hline 7 & 0.35 & 3.96 & yes & 8.18 & & A \\
\hline 8 & 1.24 & 3.87 & no & & 27.47 & B \\
\hline 9 & n.a. & n.a. & n.a. & n.a. & n.a. & n.a. \\
\hline 10 & 1.25 & 3.87 & no & & 28.37 & B \\
\hline 11 & 1.16 & 3.87 & no & & 24.10 & A \\
\hline 12 & 1.62 & 3.87 & no & & 34.91 & B \\
\hline 13 & 0.40 & 4.44 & yes & 6.92 & & B \\
\hline 14 & 1.79 & 3.87 & no & & 28.21 & B \\
\hline 15 & 1.35 & 3.87 & no & & 25.98 & B \\
\hline 16 & 0.72 & 3.87 & no & & 16.14 & B \\
\hline 17 & 2.15 & 3.87 & no & & 37.69 & A \\
\hline 18 & 2.67 & 3.87 & no & & 51.37 & A \\
\hline 19 & 1.86 & 3.87 & no & & 30.48 & B \\
\hline 20 & 0.33 & 3.61 & yes & 5.34 & & B \\
\hline 21 & 2.64 & 3.87 & no & & 52.41 & A \\
\hline 22 & 0.98 & 3.87 & no & & 19.55 & A \\
\hline 23 & 0.86 & 3.87 & no & & 15.73 & A \\
\hline 24 & 2.44 & 3.87 & no & & 36.22 & $\mathrm{C}$ \\
\hline 25 & 1.46 & 3.87 & no & & 22.05 & A \\
\hline 26 & $1: 66$ & 3.87 & no & & 28.66 & A \\
\hline 27 & 1.31 & 3.87 & no & & 27.90 & B \\
\hline 28 & 1.43 & 3.87 & no & & 20.52 & B \\
\hline 29 & 1.75 & 3.87 & no & & 29.74 & A \\
\hline
\end{tabular}


TABLE 5. SEISMIC LINE 17 , SHOTS $1-4$

\begin{tabular}{|c|c|c|c|c|c|c|}
\hline Shot & $V_{1}(\mathrm{~km} / \mathrm{s})$ & $\mathrm{V}_{2}(\mathrm{~km} / \mathrm{s})$ & Crossover? & $\begin{array}{l}\text { If Yes, Depth } \\
\text { to Basalt } \\
\text { Refractor (m) }\end{array}$ & $\begin{array}{l}\text { If No, } \\
\text { Minimum } \\
\text { Depth to } \\
\text { Refractor (m) }\end{array}$ & $\begin{array}{l}\text { Grade } \\
\text { of } \\
\text { Shot }\end{array}$ \\
\hline 1 & 0.78 & 3.87 & no & & 14.34 & B \\
\hline 2 & 2.31 & 3.87 & no & & 67.25 & B \\
\hline 3 & 0.39 & 3.87 & no & & 14.44 & A \\
\hline 4 & 0.36 & 3.87 & no & & 15.66 & A \\
\hline
\end{tabular}

TABLE 6. SEISMIC LINE 18 , SHOTS 1-18

\begin{tabular}{|c|c|c|c|c|c|c|}
\hline Shot & $V_{1}(\mathrm{~km} / \mathrm{s})$ & $V_{2}(\mathrm{~km} / \mathrm{s})$ & Crossover? & $\begin{array}{l}\text { If Yes, Depth } \\
\text { to Basalt } \\
\text { Refractor (m) }\end{array}$ & $\begin{array}{l}\text { If No, } \\
\text { Minimum } \\
\text { Depth to } \\
\text { Refractor (m) }\end{array}$ & $\begin{array}{l}\text { Grade } \\
\text { of . } \\
\text { Shot }\end{array}$ \\
\hline 1 & 0.53 & 3.87 & no & & 14.92 & $B$ \\
\hline 2 & 1.43 & 3.87 & no & & 40.48 & B \\
\hline 3 & 0.53 & 3.87 & no & & 13.93 & C \\
\hline 4 & 0.42 & 3.87 & no & & 13.39 & A \\
\hline 5 & 0.43 & 3.87 & no & & 29.91 & A \\
\hline 6 & 1.09 & 3.87 & no & & 89.18 & A \\
\hline 7 & 0.65 & 3.87 & no & & 46.40 & B \\
\hline 8 & 1.17 & 3.87 & no & & 23.08 & B \\
\hline 9 & 1.83 & 3.87 & no & & 43.23 & A \\
\hline 10 & 1.03 & 3.87 & no & & 19.95 & A \\
\hline 11 & 1.09 & 3.87 & no & & 26.03 & A \\
\hline 12 & 1.16 & 3.87 & no & & 23.55 & A \\
\hline 13 & 0.95 & 3.87 & no & & 17.83 & A \\
\hline 14 & 1.26 & 3.87 & no & & 19.84 & C \\
\hline 15 & 3.05 & 3.87 & no & & 80.80 & B \\
\hline 16 & 0.80 & 3.87 & no & & 12.33 & C \\
\hline 17 & 2.37 & 3.87 & no & & 43.80 & C \\
\hline 18 & 0.74 & 3.87 & no & & 14.96 & A \\
\hline
\end{tabular}

Lines 16, 19, 20, and 27 all trend eastward from the base of the Lava Bed Mountains towards the hand (Fig. 17, Tables 7-10, A75-78, A101, Al02-Al11, Al26A143). These data indicate that subsurface basalt is at best $15-30 \mathrm{~m}$ deep in most areas, up to a maximum depth of almost $57 \mathrm{~m}$. 
TABLE 7. SEISMIC LINE 16 , SHOTS 1-4

\begin{tabular}{lllllll}
\hline Shot & $V_{1}(\mathrm{~km} / \mathrm{s})$ & $\mathrm{V}_{2}(\mathrm{~km} / \mathrm{s})$ & Crossover? & $\begin{array}{l}\text { If Yes, Depth } \\
\text { to Basalt } \\
\text { Refractor }(\mathrm{m})\end{array}$ & $\begin{array}{l}\text { If No, } \\
\text { Minimum } \\
\text { Depth to } \\
\text { Refractor }(\mathrm{m})\end{array}$ & $\begin{array}{l}\text { Grade } \\
\text { of } \\
\text { Shot }\end{array}$ \\
\hline 1 & 0.80 & 3.87 & no & & 18.64 & $\mathrm{~A}$ \\
2 & 0.64 & 3.87 & no & & 22.18 & $\mathrm{~B}$ \\
3 & 0.41 & 3.87 & no & & 10.44 & $\mathrm{~A}$ \\
4 & 0.50 & 3.87 & no & & 18.87 & $\mathrm{~A}$ \\
\hline
\end{tabular}

TABLE 8. SEISMIC LINE 19, SHOT 1

\begin{tabular}{|c|c|c|c|c|c|c|}
\hline Shot & $V_{1}(\mathrm{~km} / \mathrm{s})$ & $V_{2}(\mathrm{~km} / \mathrm{s})$ & Crossover? & $\begin{array}{l}\text { If Yes, Depth } \\
\text { to Basalt } \\
\text { Refractor (m) }\end{array}$ & $\begin{array}{l}\text { If No, } \\
\text { Minimum } \\
\text { Depth to } \\
\text { Refractor (m) }\end{array}$ & $\begin{array}{l}\text { Grade } \\
\text { of } \\
\text { Shot }\end{array}$ \\
\hline 1 & 1.91 & 3.87 & no & & 33.55 & $\mathrm{D}$ \\
\hline
\end{tabular}

TABLE 9. SEISMIC LINE 20, SHOTS $1-10$

\begin{tabular}{|c|c|c|c|c|c|c|}
\hline Shot & $V_{1}(\mathrm{~km} / \mathrm{s})$ & $\mathrm{V}_{2}(\mathrm{~km} / \mathrm{s})$ & Crossover? & $\begin{array}{l}\text { If Yes, Depth } \\
\text { to Basalt } \\
\text { Refractor (m) }\end{array}$ & $\begin{array}{l}\text { If No, } \\
\text { Minimum } \\
\text { Depth to } \\
\text { Refractor (m) }\end{array}$ & $\begin{array}{l}\text { Grade } \\
\text { of } \\
\text { Shot }\end{array}$ \\
\hline I & 1.34 & 3.87 & no & & 17.73 & $A$ \\
\hline 2 & 1.39 & 3.87 & no & & 25.19 & B \\
\hline 3 & 0.98 & 3.87 & no & & 14.69 & B \\
\hline 4 & 0.90 & 3.87 & no & & 17.39 & $\mathrm{D}$ \\
\hline 5 & 0.97 & 3.87 & no & & 20.59 & B \\
\hline 6 & 1.54 & 3.87 & no & & 38.08 & A \\
\hline 7 & 1.92 & 3.87 & no & & 44.67 & $A$ \\
\hline 8 & 1.03 & 3.87 & no & & 21.12 & A \\
\hline 9 & 0.68 & 3.87 & no & & 20.85 & A \\
\hline 10 & 1.80 & 3.87 & no & & 40.22 & $B$ \\
\hline
\end{tabular}


TABLE 10. SEISMIC LINE 27, SHOTS 1-18

\begin{tabular}{|c|c|c|c|c|c|c|}
\hline Shot & $\mathrm{V}_{\mathrm{l}}(\mathrm{km} / \mathrm{s})$ & $V_{2}(\mathrm{~km} / \mathrm{s})$ & Crossover? & $\begin{array}{l}\text { If Yes, Depth } \\
\text { to Basalt } \\
\text { Refractor (m) }\end{array}$ & $\begin{array}{l}\text { If No, } \\
\text { Minimum } \\
\text { Depth to } \\
\text { Refractor }(\mathrm{m})\end{array}$ & $\begin{array}{l}\text { Grade } \\
\text { of } \\
\text { Shot }\end{array}$ \\
\hline 1 & 1.42 & 3.87 & no & & 20.96 & B \\
\hline 2 & 1.87 & 3.87 & no & & 21.79 & B \\
\hline 3 & 2.18 & 3.87 & no & & 26.78 & D \\
\hline 4 & 1.67 & 3.87 & no & & 21.09 & A \\
\hline 5 & 1.81 & 3.87 & no & & 27.81 & B \\
\hline 6 & 1.95 & 3.87 & no & & 34.27 & B \\
\hline 7 & 0.96 & 3.87 & no & & 18.97 & A \\
\hline 8 & 2.11 & 3.87 & no & & 56.92 & B \\
\hline 9 & 1.26 & 3.87 & no & & 31.85 & C \\
\hline 10 & 0.81 & 3.87 & no & & 20.20 & B \\
\hline 11 & 0.83 & 3.87 & no & & 16.24 & A \\
\hline 12 & 0.91 & 3.87 & no & & 23.06 & C \\
\hline 13 & 1.62 & 3.87 & no & & 36.69 & C \\
\hline 14 & 1.03 & 3.87 & no & & 25.86 & C \\
\hline 15 & 1.03 & 3.87 & no & & 18.32 & A \\
\hline 16 & 1.81 & 3.87 & no & & 33.60 & B \\
\hline 17 & 1.00 & 3.87 & no & & 17.55 & B \\
\hline 18 & 0.75 & 3.87 & no & & 16.10 & B \\
\hline
\end{tabular}

Line 28 extends from the base of the Lava Bed Mountains all the way to the western edge of the hand (Fig. 17). These data are displayed in Table 11 and Tables Al44-A173. Again, no crossovers were seen, so minimum depths to bedrock are shown. 
TABLE 11. SEISMIC LINE 28, SHOTS 1-30

\begin{tabular}{|c|c|c|c|c|c|c|}
\hline$\overline{\text { Shot }}$ & $V_{1}(\mathrm{~km} / \mathrm{s})$ & $\mathrm{V}_{2}(\mathrm{~km} / \mathrm{s})$ & Crossover? & $\begin{array}{l}\text { If Yes, Depth } \\
\text { to Basalt } \\
\text { Refractor (m) }\end{array}$ & $\begin{array}{l}\text { If No, } \\
\text { Minimum } \\
\text { Depth to } \\
\text { Refractor (m) }\end{array}$ & $\begin{array}{l}\text { Grade } \\
\text { of } \\
\text { Shot }\end{array}$ \\
\hline 1 & 1.34 & 3.87 & no & & 23.75 & $\mathrm{~A}$ \\
\hline 2 & 2.82 & 3.87 & no & & 91.46 & B \\
\hline 3 & 0.34 & 3.87 & no & & 13.92 & A \\
\hline 4 & 0.34 & 3.87 & no & & 16.32 & A \\
\hline 5 & 0.34 & 3.87 & no & & 15.41 & A \\
\hline 6 & 0.34 & 3.87 & no & & 16.46 & A \\
\hline 7 & 0.34 & 3.87 & no & & 15.26 & A \\
\hline 8 & 0.35 & 3.87 & no & & 17.26 & A \\
\hline 9 & 0.40 & 3.87 & no & & 9.41 & A \\
\hline 10 & 0.35 & 3.87 & no & & 17.27 & A \\
\hline 11 & 0.73 & 3.87 & no & & 23.54 & A \\
\hline 12 & 0.69 & 3.87 & no & & 18.61 & B \\
\hline 13 & 1.42 & 3.87 & no & & 28.07 & B \\
\hline 14 & 1.41 & 3.87 & no & & 31.84 & A \\
\hline 15 & 1.62 & 3.87 & no & & 35.24 & B \\
\hline 16 & 0.87 & 3.87 & no & & 24.47 & B \\
\hline 17 & 1.81 & 3.87 & no & & 36.82 & B \\
\hline 18 & 0.94 & 3.87 & no & & 23.34 & B \\
\hline 19 & 1.17 & 3.87 & no & & 28.23 & C \\
\hline 20 & 0.47 & 3.87 & no & & 17.47 & A \\
\hline 21 & 1.93 & 3.87 & no & & $50.4 \mathrm{I}$ & C \\
\hline 22 & 1.98 & 3.87 & no & & 50.95 & A \\
\hline 23 & 1.95 & 3.87 & no & & 49.89 & A \\
\hline 24 & 1.07 & 3.87 & no & & 24.29 & A \\
\hline 25 & 0.69 & 3.87 & no & & 20.37 & A \\
\hline 26 & 1.50 & 3.87 & no & & 34.18 & A \\
\hline 27 & 1.31 & 3.87 & no & & 30.51 & B \\
\hline 28 & 0.87 & 3.87 & no & & 24.76 & B \\
\hline 29 & 2.72 & 3.87 & no & & 71.74 & A \\
\hline 30 & 1.22 & 3.87 & no & & 25.41 & $\mathrm{~A}$ \\
\hline
\end{tabular}




\section{Seismic Lines East of the Hand}

Seismic lines $7-11,13-15$, and 21 trend eastward into the playa from the eastern edge of basalt bedrock in the hand (Fig. 17). Data from Lines 7-11 and 13 are shown in Tables 12-17, A16-A34, and A63-A66. A crossover was detected with one shot on line 12 , showing the basalt-sediment contact to be $33.76 \mathrm{~m}$ deep below that point. On seismic line 14, basalt bedrock was detected at a depth of $11.91 \mathrm{~m}$ (Tables 18, A67-A70). However, on Lines 15 and 21, no bedrock basalt was detected (Tables 19-20, A71-A74, A112-A121).

TABLE 12. SEISMIC LINE 7, SHOTS 1-9

\begin{tabular}{|c|c|c|c|c|c|c|}
\hline Shot & $\mathrm{V}_{\mathrm{l}}(\mathrm{km} / \mathrm{s})$ & $\mathrm{V}_{2}(\mathrm{~km} / \mathrm{s})$ & Crossover? & $\begin{array}{l}\text { If Yes, Depth } \\
\text { to Basalt } \\
\text { Refractor (m) }\end{array}$ & $\begin{array}{l}\text { If No, } \\
\text { Minimum } \\
\text { Depth to } \\
\text { Refractor (m) }\end{array}$ & $\begin{array}{l}\text { Grade } \\
\text { of } \\
\text { Shot }\end{array}$ \\
\hline 1 & 0.40 & 3.87 & no & & 24.44 & $A$ \\
\hline 2 & 1.58 & 3.87 & no & & 84.49 & $B$ \\
\hline 3 & 2.51 & 3.87 & no & & 99.86 & $\mathrm{C}$ \\
\hline 4 & 1.13 & 3.87 & no & & 39.96 & B \\
\hline 5 & 1.34 & 3.87 & no & & 102.43 & B \\
\hline 6 & 1.58 & 3.87 & no & & 40.06 & B \\
\hline 7 & 0.52 & 3.27 & yes & 33.76 & & B \\
\hline 8 & 0.93 & 3.87 & no & & 47.51 & B \\
\hline 9 & 1.69 & 3.87 & no & & 45.23 & $\mathrm{D}$ \\
\hline
\end{tabular}

TABLE 13. SEISMIC LINE 8, SHOTS 1-4

\begin{tabular}{lllllll}
\hline Shot & $V_{1}(\mathrm{~km} / \mathrm{s})$ & $\mathrm{V}_{2}(\mathrm{~km} / \mathrm{s})$ & Crossover? & $\begin{array}{l}\text { If Yes, Depth } \\
\text { to Basalt } \\
\text { Refractor }(\mathrm{m})\end{array}$ & $\begin{array}{l}\text { If No, } \\
\text { Minimum } \\
\text { Depth to } \\
\text { Refractor }(\mathrm{m})\end{array}$ & $\begin{array}{l}\text { Grade } \\
\text { of }\end{array}$ \\
\hline $\mathrm{l}$ & 0.38 & 3.87 & no & & 13.63 & $\mathrm{~A}$ \\
2 & 0.41 & 3.87 & no & 15.82 & $\mathrm{C}$ \\
3 & 1.49 & 3.87 & no & & 27.68 & B \\
4 & 0.72 & 3.87 & no & & 14.37 & $\mathrm{C}$ \\
\hline
\end{tabular}


TABLE 14. SEISMIC LINE 9, SHOTS $1-2$

\begin{tabular}{lllllll}
\hline Shot & $V_{1}(\mathrm{~km} / \mathrm{s})$ & $V_{2}(\mathrm{~km} / \mathrm{s})$ & Crossover? & $\begin{array}{l}\text { If Yes, Depth } \\
\text { to Basalt } \\
\text { Refractor }(\mathrm{m})\end{array}$ & $\begin{array}{l}\text { If No, } \\
\text { Minimum } \\
\text { Depth to } \\
\text { Refractor }(\mathrm{m})\end{array}$ & $\begin{array}{l}\text { Grade } \\
\text { of } \\
\text { Shot }\end{array}$ \\
\hline $\mathrm{l}$ & 0.49 & 3.87 & no & & 14.07 & B \\
2 & 0.78 & 3.87 & no & & 23.75 & A \\
\hline
\end{tabular}

TABLE 15. SEISMIC LINE 10, SHOTS 1-2

\begin{tabular}{lllllll}
\hline Shot & $V_{1}(\mathrm{~km} / \mathrm{s})$ & $\mathrm{V}_{2}(\mathrm{~km} / \mathrm{s})$ & $\begin{array}{l}\text { Crossover? } \\
\end{array}$ & $\begin{array}{l}\text { If Yes, Depth } \\
\text { to Basalt } \\
\text { Refractor }(\mathrm{m})\end{array}$ & $\begin{array}{l}\text { If No, } \\
\text { Minimum } \\
\text { Depth to } \\
\text { Refractor }(\mathrm{m})\end{array}$ & $\begin{array}{l}\text { Grade } \\
\text { of } \\
\text { Shot }\end{array}$ \\
\hline 1 & 0.41 & 3.87 & no & & 13.30 & A \\
2 & 0.41 & 3.87 & no & & 14.89 & B \\
\hline
\end{tabular}

TABLE 16. SEISMIC LINE 11 , SHOTS $1-2$

\begin{tabular}{lllllll}
\hline Shot & $V_{1}(\mathrm{~km} / \mathrm{s})$ & $V_{2}(\mathrm{~km} / \mathrm{s})$ & $\begin{array}{l}\text { Crossover? } \\
\end{array}$ & $\begin{array}{l}\text { If Yes, Depth } \\
\text { to Basalt } \\
\text { Refractor }(\mathrm{m})\end{array}$ & $\begin{array}{l}\text { If No, } \\
\text { Minimum } \\
\text { Depth to } \\
\text { Refractor (m) }\end{array}$ & $\begin{array}{l}\text { Grade } \\
\text { of } \\
\text { Shot }\end{array}$ \\
\hline 1 & 2.54 & 3.87 & no & & 34.14 & D \\
2 & 0.58 & 3.87 & no & & 14.28 & B \\
\hline
\end{tabular}

TABLE 17. SEISMIC LINE 13 , SHOTS $1-4$

\begin{tabular}{lllllll}
\hline Shot & $\mathrm{V}_{1}(\mathrm{~km} / \mathrm{s})$ & $\mathrm{V}_{2}(\mathrm{~km} / \mathrm{s})$ & Crossover? & $\begin{array}{l}\text { If Yes, Depth } \\
\text { to Basalt } \\
\text { Refractor }(\mathrm{m})\end{array}$ & $\begin{array}{l}\text { If No, } \\
\text { Minimum } \\
\text { Depth to } \\
\text { Refractor }(\mathrm{m})\end{array}$ & $\begin{array}{l}\text { Grade } \\
\text { of } \\
\text { Shot }\end{array}$ \\
\hline $\mathrm{l}$ & 1.20 & 3.87 & no & & 18.82 & $\mathrm{~A}$ \\
2 & 2.14 & 3.87 & no & & 40.42 & $\mathrm{~B}$ \\
3 & 2.11 & 3.87 & no & & 32.23 & $\mathrm{~A}$ \\
4 & 1.44 & 3.87 & no & & 21.72 & $\mathrm{~B}$ \\
\hline
\end{tabular}

TABLE 18. SEISMIC LINE 14, SHOTS $1-4$

\begin{tabular}{lllllll}
\hline Shot & $V_{1}(\mathrm{~km} / \mathrm{s})$ & $V_{2}(\mathrm{~km} / \mathrm{s})$ & Crossover? & $\begin{array}{l}\text { If Yes, Depth } \\
\text { to Basalt } \\
\text { Refractor }(\mathrm{m})\end{array}$ & $\begin{array}{l}\text { If No, } \\
\text { Minimum } \\
\text { Depth to } \\
\text { Refractor }(\mathrm{m})\end{array}$ & $\begin{array}{l}\text { Grade } \\
\text { of } \\
\text { Shot }\end{array}$ \\
\hline 1 & 1.03 & 3.87 & no & & 11.10 & $\mathrm{C}$ \\
2 & 1.09 & 3.81 & yes & 11.91 & 15.53 & $\mathrm{C}$ \\
3 & 1.10 & 3.87 & no & & 30.12 & B \\
4 & 2.01 & 3.87 & no & & & \\
\hline
\end{tabular}


TABLE 19. SEISMIC LINE 15, SHOTS $1-4$

\begin{tabular}{lllllll}
\hline Shot & $V_{1}(\mathrm{~km} / \mathrm{s})$ & $V_{2}(\mathrm{~km} / \mathrm{s})$ & Crossover? & $\begin{array}{l}\text { If Yes, Depth } \\
\text { to Basalt } \\
\text { Refractor }(\mathrm{m})\end{array}$ & $\begin{array}{l}\text { If No, } \\
\text { Minimum } \\
\text { Depth to } \\
\text { Refractor }(\mathrm{m})\end{array}$ & $\begin{array}{l}\text { Grade } \\
\text { of } \\
\text { Shot }\end{array}$ \\
\hline 1 & 0.99 & 3.87 & no & 19.73 & $\mathrm{~A}$ \\
2 & 0.68 & 3.87 & no & 17.10 & $\mathrm{~B}$ \\
3 & 0.75 & 3.87 & no & 13.67 & $\mathrm{C}$ \\
4 & 0.70 & 3.87 & no & 15.86 & $\mathrm{~B}$ \\
\hline
\end{tabular}

TABLE 20. SEISMIC LINE 21, SHOTS 1-10

\begin{tabular}{lccclll}
\hline Shot & $V_{1}(\mathrm{~km} / \mathrm{s})$ & $\mathrm{V}_{2}(\mathrm{~km} / \mathrm{s})$ & Crossover? & $\begin{array}{l}\text { If Yes, Depth } \\
\text { to Basalt } \\
\text { Refractor }(\mathrm{m})\end{array}$ & $\begin{array}{l}\text { If No, } \\
\text { Minimum } \\
\text { Depth to } \\
\text { Refractor }(\mathrm{m})\end{array}$ & $\begin{array}{l}\text { Grade } \\
\text { of } \\
\text { Shot }\end{array}$ \\
\hline $\mathrm{I}$ & 0.61 & 3.87 & no & & 13.80 & $\mathrm{~B}$ \\
2 & 0.34 & 3.87 & no & 16.89 & $\mathrm{~A}$ \\
3 & 0.40 & 3.87 & no & 9.65 & $\mathrm{~A}$ \\
4 & 0.34 & 3.87 & no & 16.78 & $\mathrm{~A}$ \\
5 & 0.34 & 3.87 & no & 15.25 & $\mathrm{~A}$ \\
6 & 0.33 & 3.87 & no & 16.57 & $\mathrm{~A}$ \\
7 & 0.33 & 3.87 & no & 15.29 & $\mathrm{~A}$ \\
8 & 0.34 & 3.87 & no & 16.24 & $\mathrm{~A}$ \\
9 & 0.36 & 3.87 & no & 13.64 & $\mathrm{~A}$ \\
10 & 0.36 & 3.87 & no & 16.50 & $\mathrm{~A}$ \\
\hline
\end{tabular}

\section{Seismic Lines South of the Hand}

Seismic lines 31-33 transect various areas south of the hand (Fig. 17). Basalt was encountered in the subsurface only at one location on line 32 at a depth of $4.81 \mathrm{~m}$ (Tables 21-23, Al79-A184). 
TABLE 21. SEISMIC LINE 31, SHOTS $1-5$

\begin{tabular}{|c|c|c|c|c|c|c|}
\hline Shot & $V_{\mathrm{I}}(\mathrm{km} / \mathrm{s})$ & $V_{2}(\mathrm{~km} / \mathrm{s})$ & Crossover? & $\begin{array}{l}\text { If Yes, Depth } \\
\text { to Basalt } \\
\text { Refractor (m) }\end{array}$ & $\begin{array}{l}\text { If No, } \\
\text { Minimum } \\
\text { Depth to } \\
\text { Refractor (m) }\end{array}$ & $\begin{array}{l}\text { Grade } \\
\text { of } \\
\text { Shot }\end{array}$ \\
\hline I & 1.06 & 3.87 & no & & 18.73 & B \\
\hline 2 & 0.78 & 3.87 & no & & 17.31 & A \\
\hline 3 & 0.95 & 3.87 & no & & 20.58 & A \\
\hline 4 & 0.46 & 3.87 & no & & 13.97 & A \\
\hline 5 & 0.34 & 3.87 & no & & 15.18 & A \\
\hline
\end{tabular}

TABLE 22. SEISMIC LINE 32, SHOTS 1-6

\begin{tabular}{|c|c|c|c|c|c|c|}
\hline Shot & $V_{1}(\mathrm{~km} / \mathrm{s})$ & $V_{2}(\mathrm{~km} / \mathrm{s})$ & Crossover? & $\begin{array}{l}\text { If Yes, Depth } \\
\text { to Basalt } \\
\text { Refractor }(\mathrm{m})\end{array}$ & $\begin{array}{l}\text { If No, } \\
\text { Minimum } \\
\text { Depth to } \\
\text { Refractor (m) }\end{array}$ & $\begin{array}{l}\text { Grade } \\
\text { of } \\
\text { Shot }\end{array}$ \\
\hline 1 & 0.63 & 3.81 & yes & 4.81 & & $\mathrm{C}$ \\
\hline 2 & 1.58 & 3.87 & no & & 22.16 & A \\
\hline 3 & 2.21 & 3.87 & no & & 33.74 & A \\
\hline 4 & 1.34 & 3.87 & no & & 23.40 & A \\
\hline 5 & 0.66 & 3.87 & no & & 9.15 & $A$ \\
\hline 6 & 0.35 & 3.87 & no & & 8.62 & D \\
\hline
\end{tabular}

TABLE 23. SEISMIC LINE 33, SHOTS 1-8

\begin{tabular}{|c|c|c|c|c|c|c|}
\hline Shot & $V_{1}(\mathrm{~km} / \mathrm{s})$ & $V_{2}(\mathrm{~km} / \mathrm{s})$ & Crossover? & $\begin{array}{l}\text { If Yes, Depth } \\
\text { to Basalt } \\
\text { Refractor (m) }\end{array}$ & $\begin{array}{l}\text { If No, } \\
\text { Minimum } \\
\text { Depth to } \\
\text { Refractor }(\mathrm{m})\end{array}$ & $\begin{array}{l}\text { Grade } \\
\text { of } \\
\text { Shot }\end{array}$ \\
\hline 1 & 2.51 & 3.87 & no & & 36.20 & A \\
\hline 2 & 2.04 & 3.87 & no & & 33.08 & B \\
\hline 3 & 1.37 & 3.87 & no & & 25.14 & B \\
\hline 4 & 2.31 & 3.87 & no & & 58.05 & A \\
\hline 5 & 2.27 & 3.87 & no & & 68.46 & B \\
\hline 6 & 0.80 & 3.87 & no & & 31.03 & A \\
\hline 7 & 0.33 & 3.87 & no & & 15.11 & $A$ \\
\hline 8 & 0.33 & 3.87 & no & & 16.67 & A \\
\hline
\end{tabular}




\section{Seismic Lines Across the Hector Mine Rupture}

Seismic line 22 was run across the Hector Mine rupture where no subsurface basalt was detected (Fig. 17). No crossovers were identified (Tables 24, Al22-A125).

TABLE 24. SEISMIC LINE 22, SHOTS 1-4

\begin{tabular}{|c|c|c|c|c|c|c|}
\hline Shot & $\mathrm{V}_{\mathrm{l}}(\mathrm{km} / \mathrm{s})$ & $V_{2}(\mathrm{~km} / \mathrm{s})$ & Crossover? & $\begin{array}{l}\text { If Yes, Depth } \\
\text { to Basalt } \\
\text { Refractor (m) }\end{array}$ & $\begin{array}{l}\text { If No, } \\
\text { Minimum } \\
\text { Depth to } \\
\text { Refractor (m) }\end{array}$ & $\begin{array}{l}\text { Grade } \\
\text { of } \\
\text { Shot }\end{array}$ \\
\hline I & 0.33 & 3.87 & no & & 15.07 & A \\
\hline 2 & 1.91 & 3.87 & no & & 66.47 & A \\
\hline 3 & 0.34 & 3.87 & no & & 15.19 & A \\
\hline 4 & 0.34 & 3.87 & no & & 16.73 & A \\
\hline
\end{tabular}

\section{Seismic Lines Across a Major Stepover in the Rupture}

Seismic line 35 was run across a major stepover in the Hector Mine rupture, where the Lavic Lake fault makes a prominent surface step to the left (Fig. 17). This is the same fault jog where paleoseismic trenches were dug by Rymer and others, and where magnetometer line 25 crosses the fault rupture. Subsurface basalt was detected on one shot, at a depth of $13.18 \mathrm{~m}$ (Tables 25, A193-A195).

TABLE 25. SEISMIC LINE 35, SHOTS $1-3$

\begin{tabular}{lllllll}
\hline Shot & $V_{1}(\mathrm{~km} / \mathrm{s})$ & $V_{2}(\mathrm{~km} / \mathrm{s})$ & Crossover? & $\begin{array}{l}\text { If Yes, Depth } \\
\text { to Basalt } \\
\text { Refractor }(\mathrm{m})\end{array}$ & $\begin{array}{l}\text { If No, } \\
\text { Minimum } \\
\text { Depth to } \\
\text { Refractor }(\mathrm{m})\end{array}$ & $\begin{array}{l}\text { Grade } \\
\text { of } \\
\text { Shot }\end{array}$ \\
\hline 1 & 0.37 & 3.52 & yes & 13.18 & & B \\
2 & 3.27 & 3.87 & no & & 317.56 & C \\
3 & 2.54 & 3.87 & no & & 167.03 & C \\
\hline
\end{tabular}




\section{DGPS/GIS Data}

Using ArcView 3.2a, a geologic map was generated of the field area (Fig. 20). The 3D DEM generated of the field area is shown in a three-dimensional view in Figure 36. A 3D DEM was also generated that shows minimum and calculated depths to basalt bedrock beneath the field area (Fig. 37). The reader is invited to view these figures on the included disk for increased resolution. 


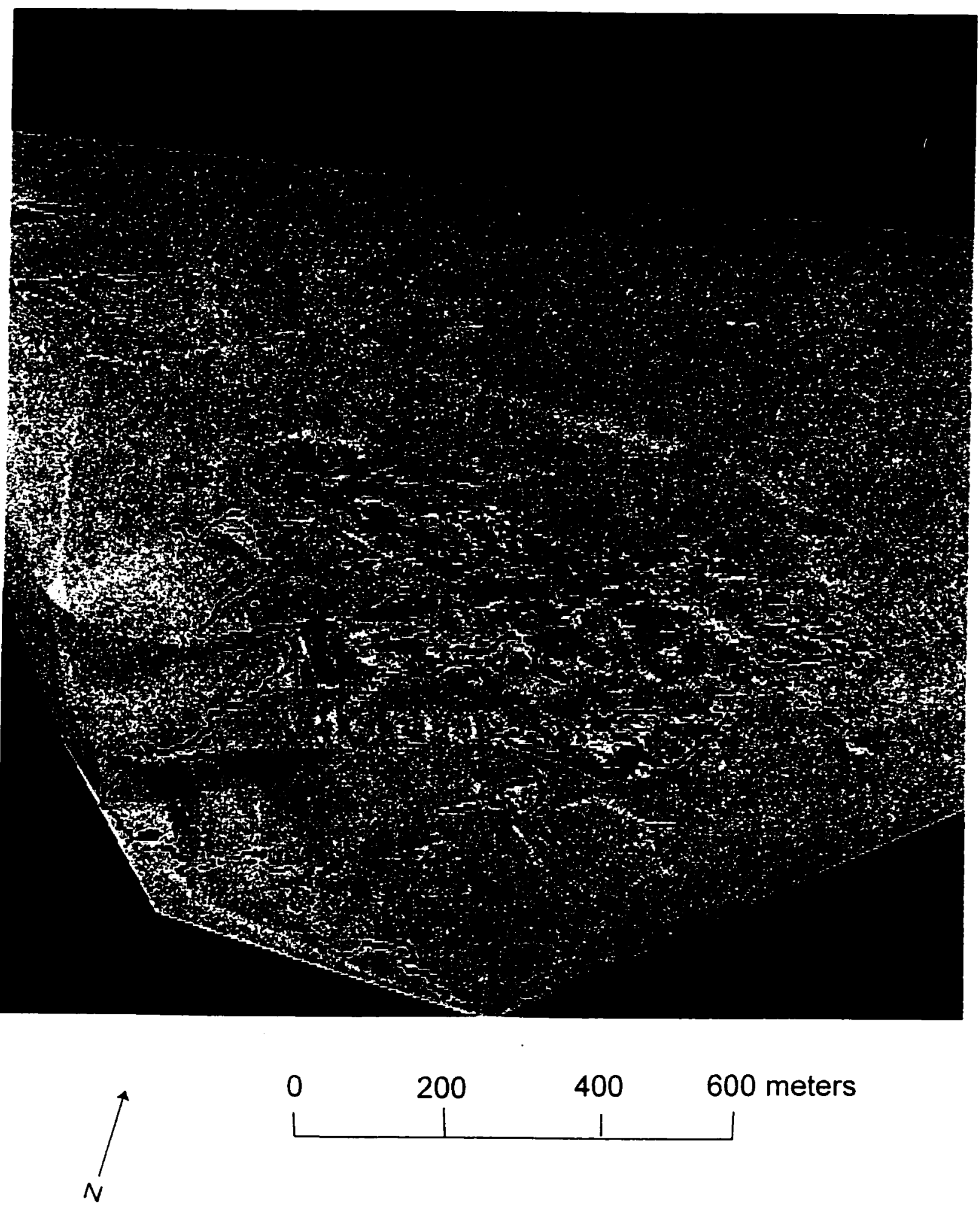

Figure 36. Three-dimensional DEM of the field area, showing the extent of major units. The Hector Mine rupture is shown in red, the edge of thrust sheets are shown in blue, and the basalt-sediment contact is shown as a heavy black line. This figure was generated by rover files imported into ArcView 3.2a. 


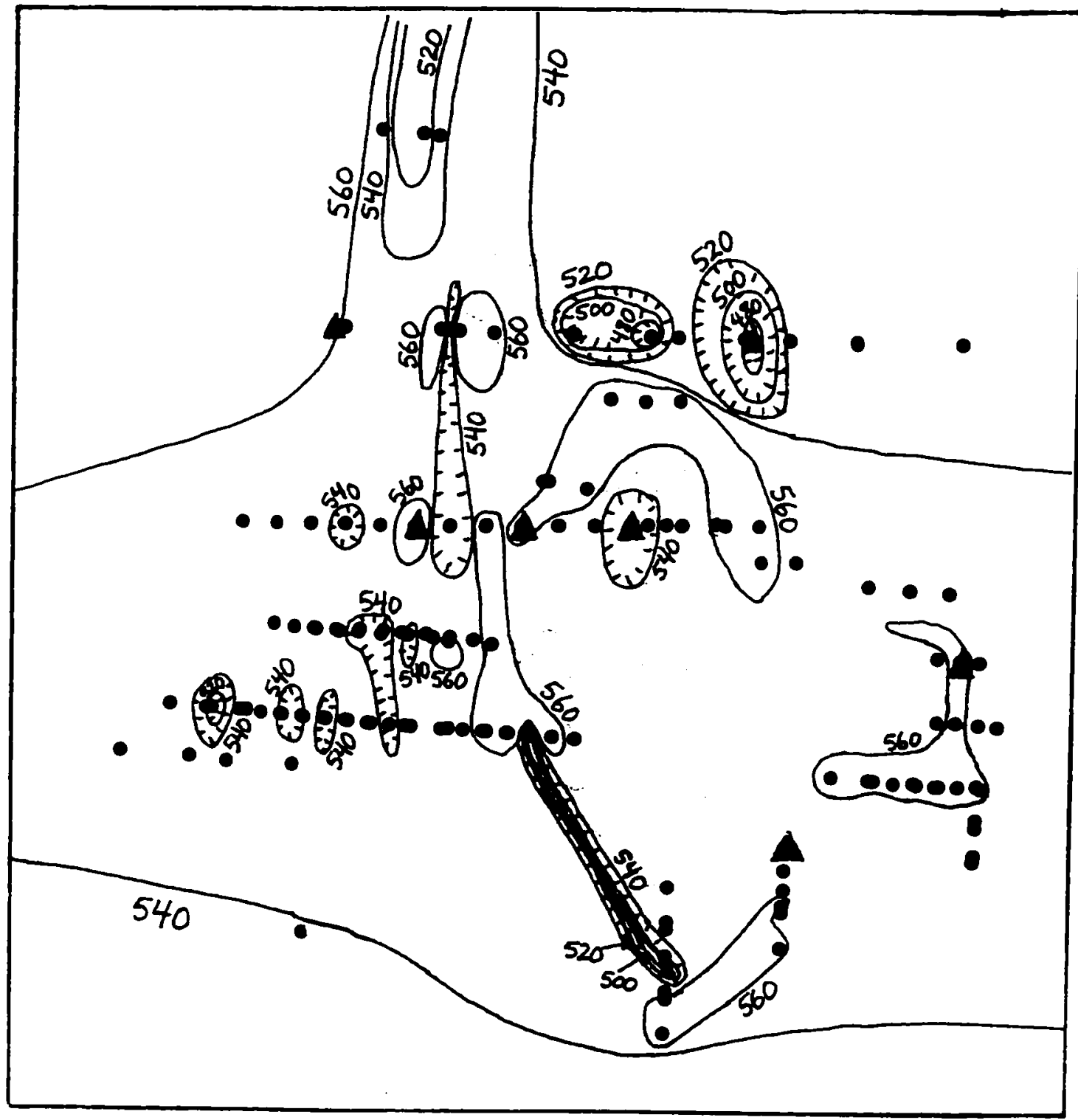

LEGEND

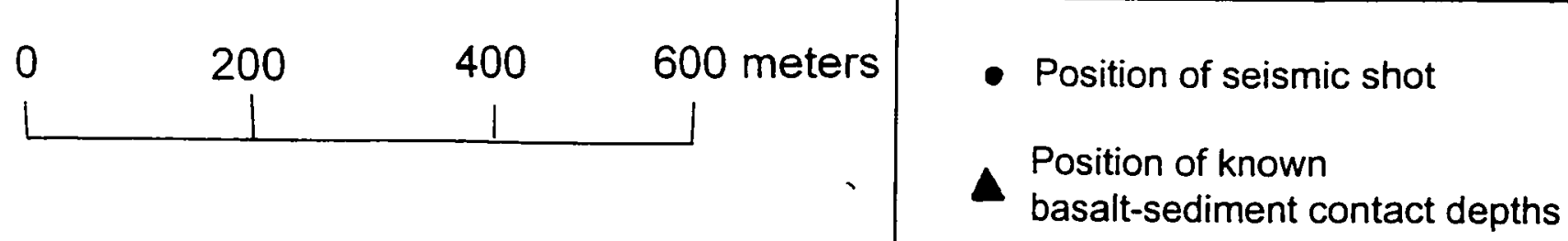

Figure 37. Contour map of minimum or calculated basalt-sediment contact elevations (in meters) in the field area. The contour interval is $20 \mathrm{~m}$, and the base contour is $400 \mathrm{~m}$. Portions of this figure were generated by rover files imported in ArcView 3.2a. 


\section{DISCUSSION}

\section{Depth to the Basalt-Sediment Contact in the Subsurface}

In most seismic lines west (Tables 1-6, 11), east (Tables 12-20), and south (Tables 21-23) of the hand, basalt could not be detected in the subsurface. The absence or considerable depth of the basalt on these seismic lines supports the interpretation that the hand is isolated from the main lava flow on the Lava Bed Mountains.

Basalt crossovers were found only in eight shots, four of which were located west of the hand (lines 2 and 12), two to the east of the hand (lines 7 and 14), one to the south of the hand (line 32), and one under a major stepover (line 35). The depth of the basaltsediment contact ranged from a shallow $3.97 \mathrm{~m}$ west of the hand to $33.76 \mathrm{~m}$ east of the hand.

The contour map showing minimum and calculated basalt-sediment contact elevations in the subsurface (Fig. 37) shows a local minimum (408 m elevation) of the minimum basalt-sediment contact elevation directly west of the base of the finger. Other lows are present near the east of the tip of the finger ( $476 \mathrm{~m}$ elevation) and west of the hand (485 $\mathrm{m}$ elevation). These results imply that basalt either is not present or lies at considerable depths in these areas.

The shape of the curves in the various magnetic anomaly graphs from the linear magnetic surveys may offer clues to the depth of basalt bedrock in various areas of the playa (Breiner, 1973). Curves in a variety of broad to tight shapes suggest that the basalt may be found at a variety of depths in the subsurface. For example, on line 1, at $1000 \mathrm{ft}$. a very tight curve in the graph shows that basalt could be relatively close to the surface 
(Fig. 24). On the same graph, a broad curve from $1250 \mathrm{ft}$. to $1700 \mathrm{ft}$. could indicate that basalt is found at great depths. However, these relations do not address the shape or orientation of the body affecting the anomalies. Furthermore, although basalt is the only factor discussed here, other buried features could affect the magnetic anomaly, including buried metal, military equipment, void spaces, or other unknown objects.

\section{Geometry of the Basalt-Sediment Contact in the Subsurface}

Because basalt bedrock was encountered in some lines but was absent or too deep to detect in others, the basalt-sediment contact could be highly irregular in the subsurface (Tables 1-25). This topography could be very similar to the surface topography of the hand, the frisbee, and the base of the Lava Bed Mountains. The erratic patterns of the magnetic contours in Figure 23 also indicate that the basalt could have an irregular subsurface topography. The DGPS/GIS DEMs and maps produced in this study clearly illustrate the presence of anomalous local highs in the topography of the hand and frisbee. This irregular topography is unlikely to be primary morphology of an undisturbed lava flow, suggesting post-flow disturbances from fault movement, local subsidence, cracking, slumping, or exploding ordnance. It is also important to note that irregular subsurface and surface topography could also be the result of erosion due to pre-playa sedimentation processes. such as lacustrine erosion, repeated burial, or erosion due to possible exposure of the basalt to other processes which have occurred over the last 600,000 years.

Although basalt could not be detected on most of the seismic lines, it may be present at depths greater than the resolution of the instruments used. This may indicate that the hand and frisbee are, in fact, isolated from the main flow of the Sunshine Peak 
basalt, separated only by thick deposits of playa sediment. This apparent isolation of the hand and frisbee by playa sediment could be the result of faulting or even a volcanic fissure, which various kinematic models attempt to address in later sections.

\section{The Basalt-Sediment Subsurface Contact in Relation to the Hector Mine Rupture}

The seismic line across a linear part of the Hector Mine rupture revealed no basalt in the subsurface within the depth limits of the survey (Table 24). However, basalt bedrock was encountered on a line across the major left-step in the rupture to the north of the hand (Table 25). In line 1, the anomaly remains negative but clearly fluctuates near the rupture, suggesting a magnetic source in the subsurface. In lines 3 and 4, a magnetic source causes a sharp increase in the magnetic anomaly in the center of the rupture area, produced by a magnetic source that could be a buried extension of the finger. The negative anomalies surrounding this sharp increase are probably related to thick playa sediment cut by the fault. In line 23 , a sharp decrease in the magnetic anomaly around the rupture indicates an absence of a magnetic body around the subsurface rupture. However, in lines 24-26, another magnetic source sharply increases the magnetic anomaly around the rupture area. Lines 25 and 26 of this group cross a major left step. Thus, whatever caused the increase in the magnetic anomaly may also have caused the rupture to form the left step.

These observations suggest that, where basalt is uncommon or absent in the subsurface, the Hector Mine rupture in Lavic Lake is linear (or dominated by right-lateral en-echelon tension gashes). In other words, the rupture would probably tend to propagate 
in a pattern dictated by the properties of the dry clay and sand of the playa. However, where basalt is present in the subsurface, the rupture steps left (or right, although it has not been observed in this study), creating positive (or negative) flower structures, respectively. The presence of restraining bends in the rupture near the hand and frisbee indicates that the basalt may direct propagation of fault rupture. The hand and frisbee may be positive flower structures produced by multiple earthquakes at such restraining bends. This interpretation has other implications that will be discussed later in several kinematic models.

\section{Geomorphic Features of the Sunshine Peak Flow and Lavic Lake Playa}

Field observations and previous research indicate that not all features on the hand, frisbee, and Sunshine Peak flow on the Lava Bed Mountains are related to movement on the Lavic Lake fault. Basaltic stone pavement, or desert pavement, is very common on the surface of the hand and frisbee, and in many places on the playa. Wells et al. (1995) presented a soil development model, based on young basalts in the Mojave Desert, to explain how these unusual features form. As the surface of a low-relief basalt flow is exposed to eolian weathering, wind-blown sediment and shrinking and swelling of soil horizons lifts basalt clasts and other material vertically away from bedrock. This process eventually forms an accretionary mantle, or package of wind-blown sediment, between the pavement and the substrate. This package of sediment is generally more poorly sorted than typical playa sediment, which consists of mostly clay-sized particles (Wells et al., 1995). Therefore, the mere presence of basalt in the field area may indicate that some 
poorly sorted or denser subsurface layers may also be present, consistent with a typical desert soil-development profile.

\section{Kinematic Models}

Various kinematic models may explain the observations presented in this study. Each of these proposed models uses basic physical or geological principles to explain how these complex features could have formed from young volcanic eruptions, fault movement, principal stress directions, and regional tectonics.

\section{The Fissure Flow Model}

The basic premise of this model is that the Lavic Lake fault was, and continues to be, a vertical conduit or "path of least resistance" for rising basaltic magma in this section of the Mojave Desert. This implies that in the recent geologic past, perhaps no earlier than the Pleistocene, the Lavic Lake fault zone and the Pisgah fault zone were conduits for fissure flows, following the approximate strike of the two faults. A kinematic model for this process is shown in Figure 38. At time 1, faulting occurs with no surface basalt

present. At time 2, basaltic lavas are erupted. At time 3, subsequent faulting alters the subsurface and surface topography.

This model is supported by several lines of evidence:

1. In most areas near the hand, basalt bedrock could not be found within detection limits, indicating it is found at considerable depths or is completely absent. This distribution is more consistent with near-vertical emplacement of basalt in a fissure than eruption of a surficial lava flow with a gentle slope. 


\section{Time 1 (>1 mya)}

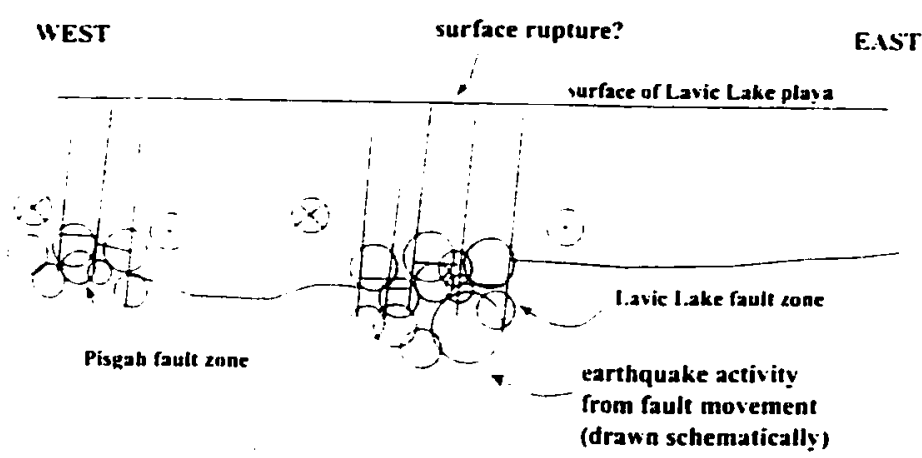

\section{Time 2 (0.8-0.5 mya)}

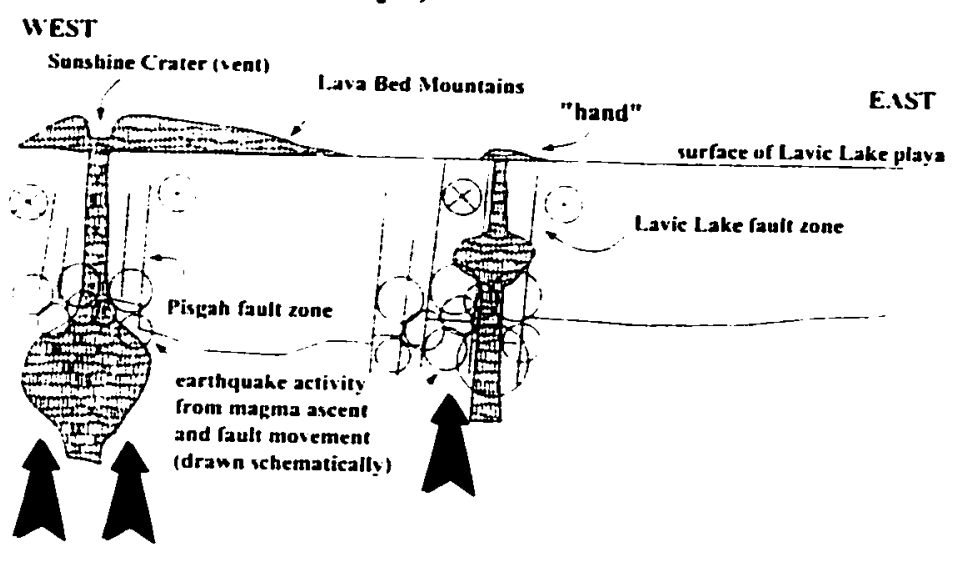

\section{Time 3 (0.5 mya-present)}

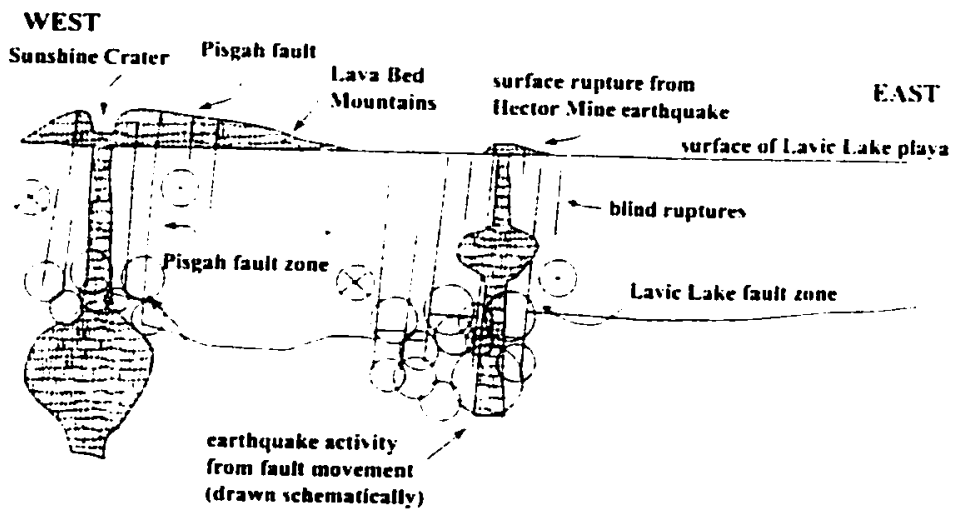

Figure 38. Times 1, 2, and 3 for the fissure flow model (generalized). 
2. The spatial correlation of the Lavic Lake fault and the basalt of the hand suggests that the basalt and the fault may be genetically related. The same is true of the Pisgah fault, which strikes directly through the Lava Bed Mountains.

3. The overall trend of the Pisgah lava flow and the Lava Bed Mountains is roughly northwest, parallel to the strikes of most of the strike-slip faults in this region, including the Pisgah and Lavic Lake faults. Both faults may have controlled where and how the basalt was erupted.

However, this model.has inconsistencies that were not resolved by this study.

1. Basalt encountered in the subsurface between the hand and the Lava Bed Mountains shows that not all the basalt in the subsurface is found at considerable depths in this region. These exceptions are difficult to explain as products of fissure flows, because they do not occur along active faults.

2. The methods used in this study cannot identify the nature of subsurface materials beneath the hand and frisbee. If such materials are sedimentary, the hand and the frisbee could be large collections of basalt "exhumed" or isolated from the original lava flow.

3. Available data are insufficient to determine whether the irregular topography in the basalt's surface and near-surface contact is a result of normal lava intrusion or multiple faulting events.

4. The overall northwest trend of the Pisgah lava flow and the Lava Bed Mountains could be coincidental, rather than fault-controlled.

\section{The Multiple Dip-Slip Thrusting Model}

This model attributes the hand and the frisbee not to the eruption of a fissure flow, 
but to the combined movement of many earthquakes, comparable in size and amount of surface slip with the Hector Mine and Landers earthquakes. Because the basalt has an age of approximately 0.6 Ma (A. Glazner, personal communication, 2000), and the Lavic Lake fault has an unknown, but long recurrence time (M. Rymer, personal communication), many surface-rupturing earthquakes would have to occur in this model. At time I (Fig. 39), the lava in the Lava Bed Mountains erupts from the Sunshine and Pisgah craters (the latter not shown in figure). At time 2, large earthquakes produce ruptures that cut solidified lava flows. This surface faulting has components of both strike-slip movement and thrusting on both sides of the hand and frisbee as observed in the Hector Mine surface rupture. Thrusting forms in stepovers, where the hand and frisbee are located. At time 3, between faulting events, playa depositional processes occur to form Lavic Lake playa. Events portrayed at time 2 and time 3 alternate until the present, defining what is now known as the hand and frisbee (Fig. 39).

The evidence to support this model is as follows:

1. Field observations unequivocally support the occurrence of a minor component of thrusting along the flanks of both the hand and the frisbee along the Hector Mine rupture. These observations include the presence of basalt thrust across the fault rupture, thrusts within playa sediment, and compressional ridges. These structures show thrust movement towards the east and west. and are found predominantly around the hand and frisbee.

2. The irregular surface topography between the hand and the Lava Bed Mountains indicates that faulting also may have occurred in that region. Such hypothetical 
Time 1 (1.0-0.8 mya)

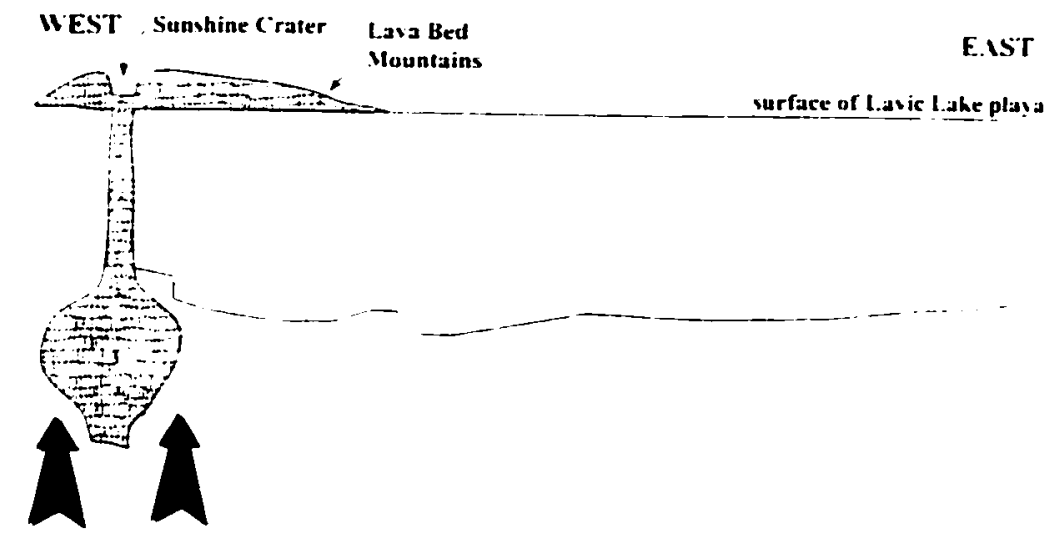

Time 2 (0.8-0.5 mya)

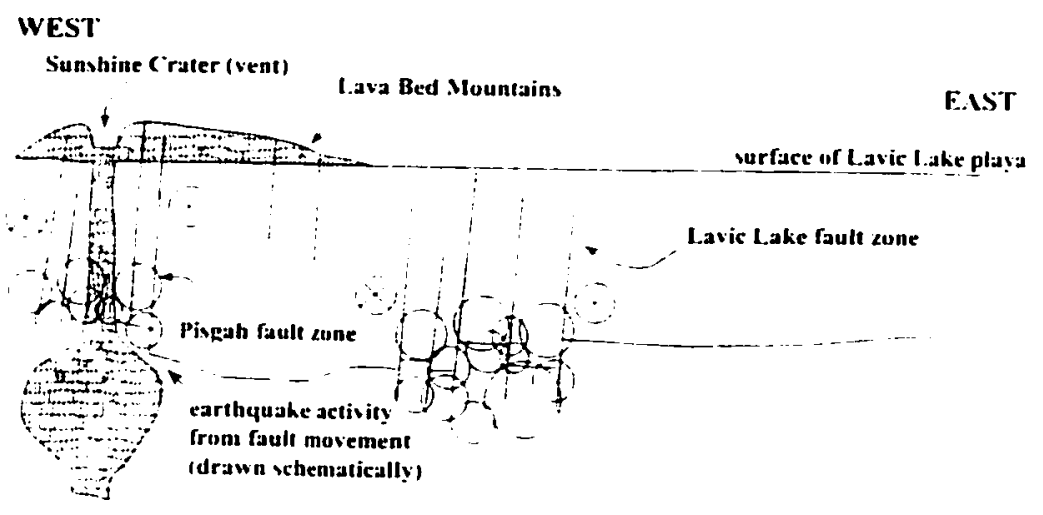

\section{Time 3 (0.5 mya-present)}

WEST

Suashine Crater (went) Hector Mine rupture
"hand"

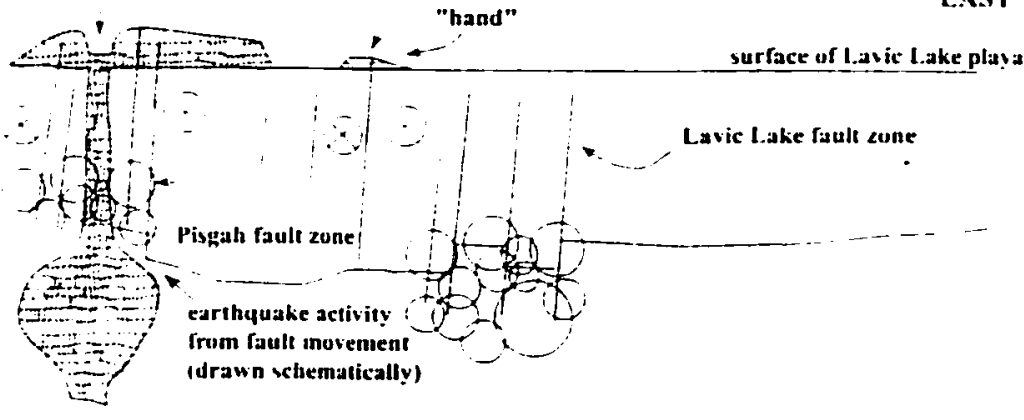

Figure 39. Times 1, 2, and 3 for the multiple dip-slip thrusting model (generalized). 
subsidiary faults may have aided in the upward movement of the hand and the frisbee.

However, the problems with this model make it less likely to explain the local geology.

1. The amount of thrusting produced during the Hector Mine earthquake is too small. Assuming the 1999 rupture is typical, and given the assumed recurrence interval of the Lavic Lake fault and other faults of the ECSZ, thousands of earthquakes would have to occur to produce the amount of separation between the hand and the Lava Bed Mountains.

2. The model assumes that thrust movement occurred in the majority of the past earthquakes. There is no evidence that this is the case.

\section{The Basalt Exhumation Model}

This model explains the formation of the hand and the frisbee as the latest in a series of stages involving vertical movement (exhumation) of large basalt blocks from the original lava flows. This model also involves the combined effects of many earthquakes comparable to the size of the Hector Mine or Landers earthquakes since the basalt was erupled. At time I (Fig. 40), the lava in the Lava Bed Mountains erupts from the Sunshine and Pisgah craters (the latter not shown in figure). At time 2, eolian processes bury portions of the new lava flow with sand and clay. At time 3, faulting occurs from sizable earthquakes, cutting the solidified lava flows and playa sediment. This faulting has components of both strike-slip movement and thrusting towards the east, as observed in the Hector Mine surface rupture. Buried basalt continues to crack from weathering. At time 4 , in areas where the fault produces a left step, large basalt boulders are vertically 


\section{Time 1 (1.0-0.8 mya)}
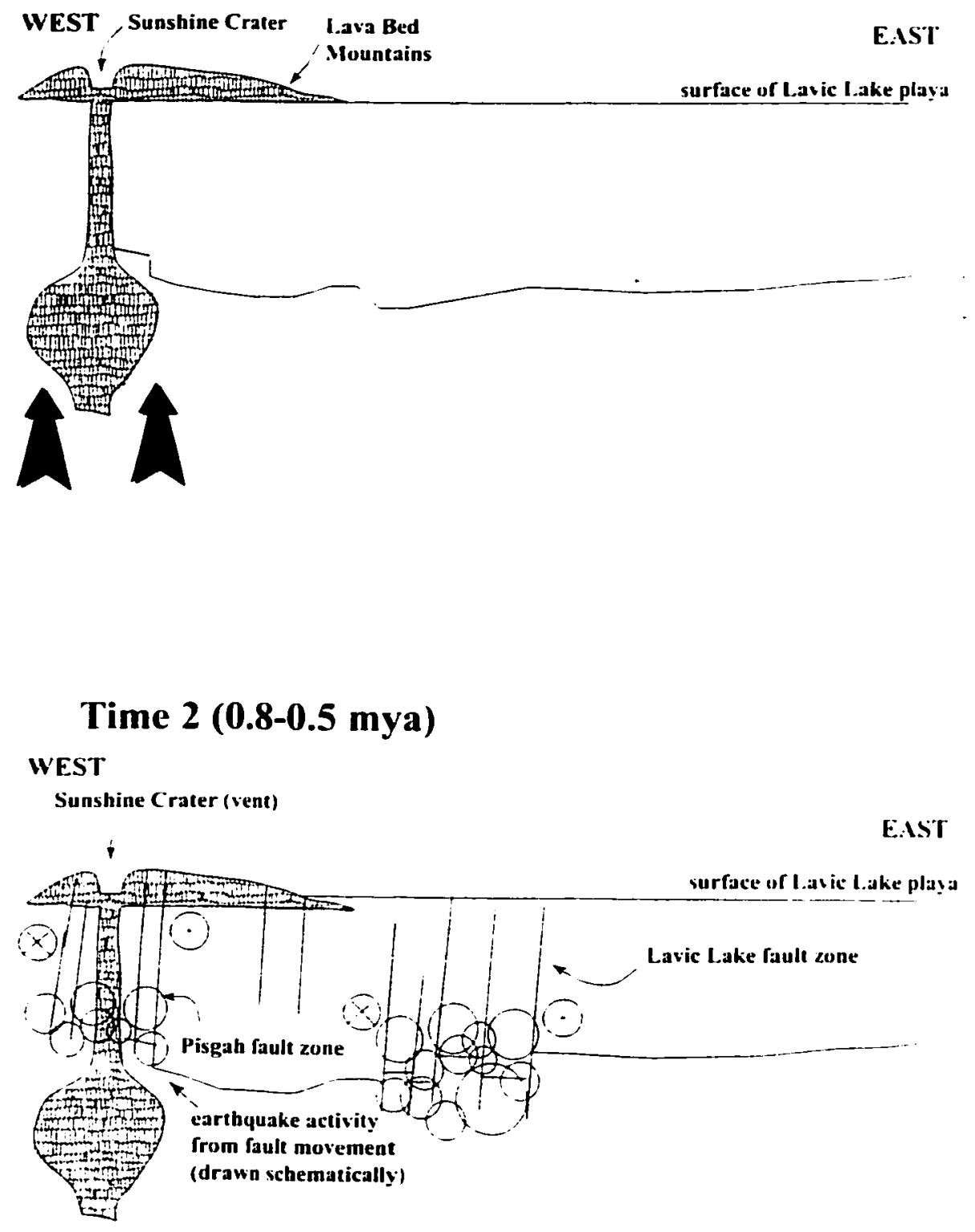

Figure 40. Times 1, 2, 3, and 4 for the basalt exhumation model. 


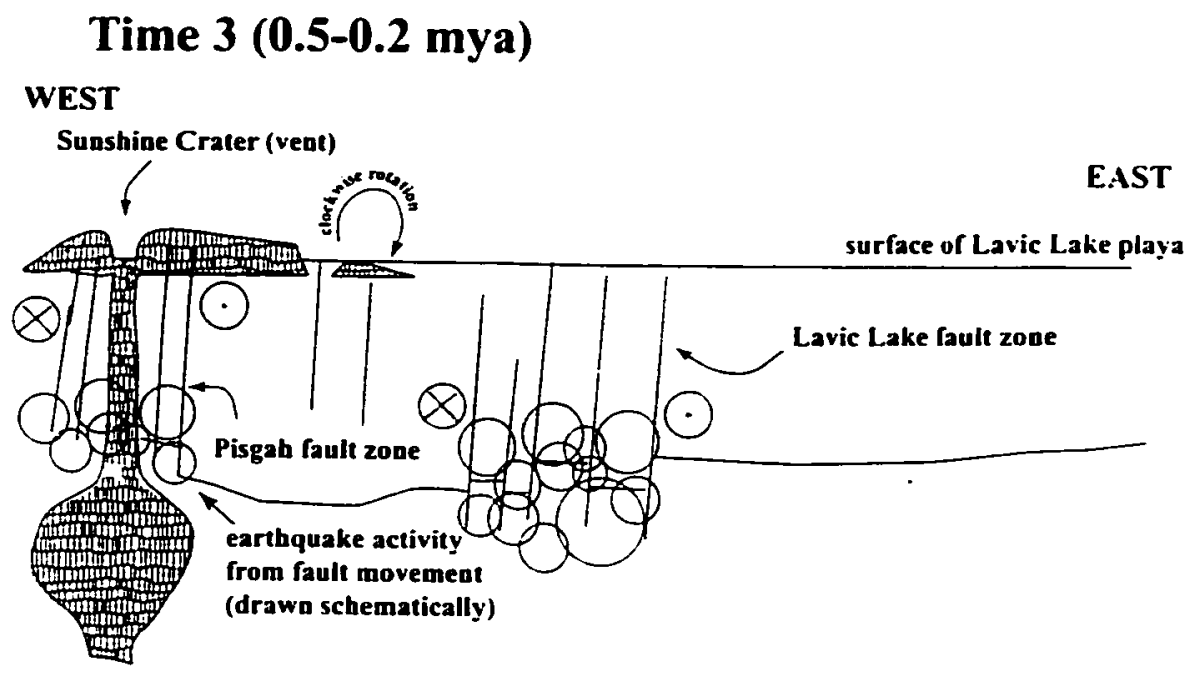

\section{Time 4 (0.2 mya-present)}

\section{WEST}

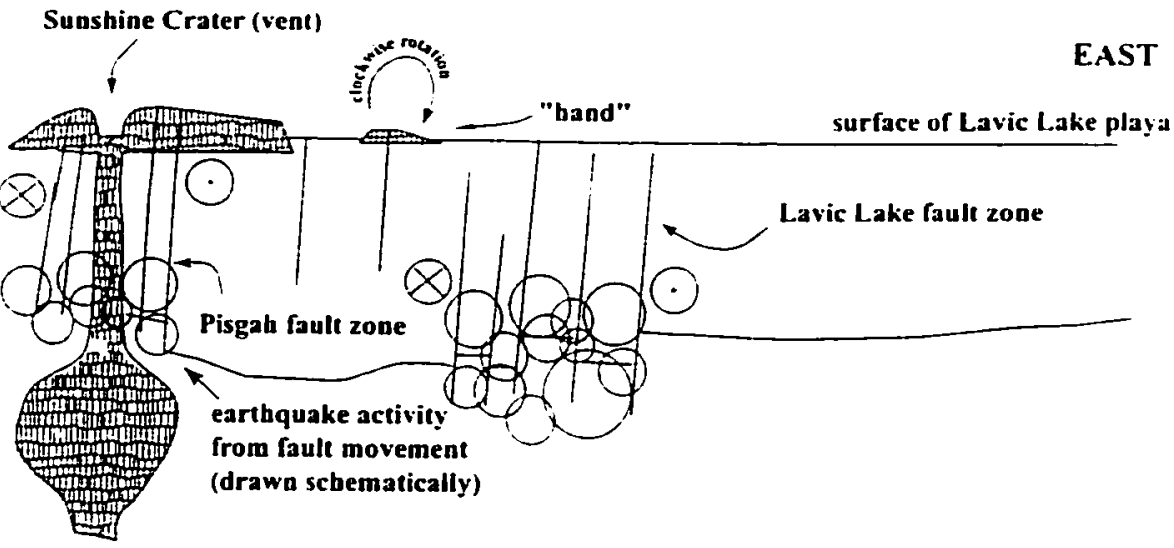

Figure 40 (continued). Times 1. 2. 3. and 4 for the basalt exhumation model. 
lifted and rotated. Events portrayed at time 3 and time 4 alternate repeatedly until the present, defining what is now known as the hand and frisbee.

The evidence to support this model is as follows:

1. A minor component of thrusting accompanied the Hector Mine earthquake, best shown in the hand and the frisbee, where the Lavic Lake fault makes a left (restraining) step. Repeated earthquakes with similar slip could lift the basait found in the hand and frisbee several tens of meters from the original lava flow below.

2. Seismic line 35 positively detected a buried basalt structure under a major stepover in the rupture, north of the hand.

However, some evidence refutes this model:

1. There is no way to determine whether the basalt that makes up the hand and the frisbee is underlain by playa sediments or basalt bedrock. The latter would support a fissure flow model, discussed in the previous section. The former would support this model, showing that the hand and frisbee are indeed large collections of basalt being "exhumed" or isolated from the original lava flow.

2. The model assumes a thrust component in the majority of the past earthquakes, but such slip is indeterminate.

\section{Error Analysis}

\section{Magnetic Surveys}

The magnetic surveys in this study were subject to several sources of uncertainty or error. Multiple readings of the magnetic field taken at one point on the surface varied by a few $\mathrm{nT}$ to a few hundred $\mathrm{nT}$ despite the short time (several seconds) elapsed between 
readings. The magnetic readings presented here represent the final reading in a series of three taken at one point, rather than an average of all three.

The magnetic data show a bull's eye pattern on the contour map generated by ArcView 3.2a. This pattern is due to aliasing of the magnetic data in map areas that lacked sufficient data. In order to correct this alias, more data should be collected to fill in obvious "gaps."

The IGRF model used in the magnetic field corrections only employs a few variables in the projected calculation of the magnetic field at a particular point on Earth's surface: latitude, longitude, and elevation up to two decimal places. Given the relatively small field area in this study, greater precision in the magnetic field is needed to show more detailed variations in the magnetic field. This precision cannot be given by the IGRF model. This imprecision is also carried into the calculations of diumal variation.

\section{Seismic Refraction Surveys}

Seismic refraction surveys incorporated sufficient uncertainties. During the collection of most of the data, geophones 8,9 , and 11 proved inoperable and did not yield reliable data. Most lines were overlapped to attempt to correct for this problem. Also, when a shot was performed and the seismic waves were displayed, arrival times could only be determined to the nearest $0.4 \mathrm{~ms}$.

In many areas of the playa, seismic refraction proved to be difficult to implement, given the unconsolidated and desiccated nature of the playa sediments. Where geophones could not be inserted into the sediment firmly, or were inserted inside vertical or semi-vertical cracks, recorded arrival times may have been earlier or later than the true 
arrival times.

Multiple interpretations of the seismic data are possible. For example, different researchers could have different opinions about the location of a crossover for a particular seismic shot, or where to pick an arrival time on a geophone's waveform. Data presented here represent the best interpretation of these aspects.

\section{DGPS/GIS Data}

Even with the removal of selective availability, all satellite data collected in this study have many sources of error that can be the result of the DGPS unit or tropospheric aberrations. Inconsistencies in the elevation or position of the base station can yield GPS positions that are consistently inaccurate. Inaccurate base station files used in the differential corrections of all the GPS data can make the entire data set slightly inaccurate. Furthermore, due to the fact that the base station is located almost $400 \mathrm{~km}$ away from Lavic Lake, accuracy of the spatial data can be affected because of a possible difference of space vehicles in view at the field area and at the base station an given time. 


\section{CONCLUSION}

After the Landers and Hector Mine earthquakes, two prominent earthquakes in the ECSZ, this study was conducted in part to aid in our understanding of the Lavic Lake fault's previous activity in this section of the Mojave Desert. Seismic refraction, magnetics, and DGPS/GIS systems were all utilized in various aspects of this study. Seismic refraction and magnetic results suggest that the basalt buried beneath various sections of Lavic Lake playa has an irregular topography, not consistent with an undisturbed lava flow. The exact depths and orientations of this buried basalt-sediment interface could not be determined by these methods. Magnetics and seismic refraction confirmed the presence of anomalous features (perhaps basalt) near the Lavic Lake fault zone that ruptured in the Hector Mine earthquake. Seismic refraction revealed the possibility that the deep burial or absence of basalt somehow influenced the formation of prominent left steps and linear sections of the Lavic Lake fault zone. It is safe to conclude that most basalt is found at considerable depths below many sections of Lavic Lake playa between the hand and the Lava Bed Mountains.

These observations lead to several models to describe the local geology. The most likely model, dubbed the fissure flow model, considers the depth of the basalt and suggests that the Lavic Lake fault once acted as a conduit for escaping basaltic lava, which created the hand and frisbee. Subsequent faulting has since altered the surface topography. Other less likely models include the dip-slip thrusting and basalt exhumation models. Clearly, more work in this area needs to be completed to further understand which of these models is the most realistic. 


\section{REFERENCES CITED}

Breiner, S., 1973, Applications manual for portable magnetometers: Sunnyvale, CA, GeoMetrics Publications, $58 \mathrm{p}$.

Department of Conservation, California Division of Mines and Geology (State of California Resources Agency), 1988, Aeromagnetic map of the San Bernardino quadrangle: Regional Geophysical Map No. 3D, scale 1:250,000.

Dibblee, T. W. Jr., 1966, Geologic map of the Lavic quadrangle, San Bernardino County, California: United States Geological Survey Miscellaneous Investigations Map I472 , scale $1: 62,500$.

International Association of Geomagnetism and Aeronomy (IAGA), Division V, Working Group 8, 2000, International Geomagnetic Reference Field - Epoch 2000: Revision of the IGRF for 2000-2005 (updated 5/26/00): online, 3 p. http://www.ngdc.noaa.gov/[AGA/wg8/igrf2000.html

Jagiello, K., Christie, J. M., and Blom, R. G., 1992, Horizontal separation of major late Cenozoic strike-slip faults in the Twentynine Palms region, Mojave Desert, California, in Richard, S. M., ed., Deformation associated with the Neogene Eastern California Shear Zone, southwestern Arizona and southeastern California: Redlands, California, San Bernardino County Museum Special Publication, p. 4853.

Miller, M. M., Johnson, D. J., Dixon, T. H., and Dokka, R. K., 2001, Refined kinematics of the Eastern California shear zone from GPS observations, 1993-1998: Journal of Geophysical Research, v. 106, p. 2245-2263.

Rymer, M. J., Seitz, G. G., Weaver, K. D., Orgil, A., Faneros, G., Hamilton, J. C., and Goetz, C., 2002, Geologic and paleoseismic study of the Lavic Lake fault at Lavic Lake playa, Mojave Desert, southern Califormia: Bulletin of the Seismological Society of America, v. 92, in press.

Ross, T. M., 1992, Geologic and paleomagnetic constraints on the timing of initiation and amount of slip on the Rodman and Pisgah faults, central Mojave Desert, California, in Richard, S. M., ed., Deformation associated with the Neogene Eastern California Shear Zone, southwestern Arizona and southeastern California: Redlands, California, San Bernardino County Museum Special Publication, p. 4853. 
Scientists from the U.S. Geological Survey, Southern California Earthquake Center, and the California Division of Mines and Geology (USGS, SCEC, and CDMG), 2000, Preliminary report on the 16 October 1999 M7.1 Hector Mine, California, earthquake: Seismological Research Letters, v. 71, p. 11-23.

Treiman, J. A., Kendrick, K. J., Bryant, W. A., Rockwell, T. K., and McGill, S. F., 2002, Primary surface rupture associated with the $M_{w} 7.116$ October 1999 Hector Mine earthquake, San Bernardino county, California: Bulletin of the Seismological Society of America, v. 92, in press.

Wells, S. G., McFadden, L. D., Poths, J., and Olinger, C. T., 1995, Cosmogenic ${ }^{3} \mathrm{He}$ surface-exposure dating of stone pavements: Implications for landscape evolution in deserts: Geology, v. 23, p. 613-616.

Wise, W. S., 1966, Geologic map of the Pisgah and Sunshine Cone lava fields: National Aeronautics and Space Administration Technical Letters, v. 11,8 p. 


\section{APPENDICES}

Summaries of Data

Table A1. Summary of Magnetometer Data Tables A2-A195. Summary of Seismic Refraction Data 


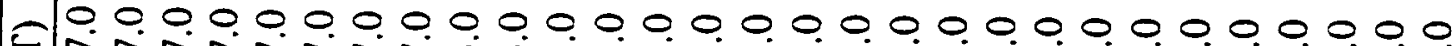

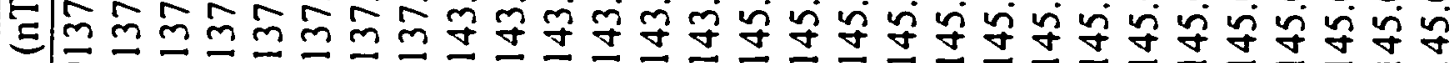

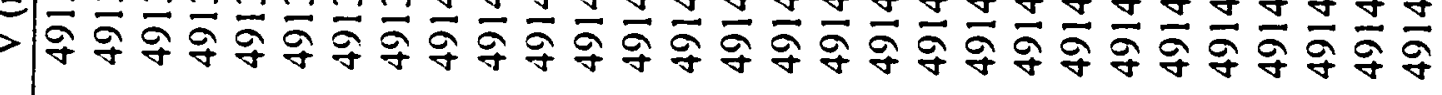

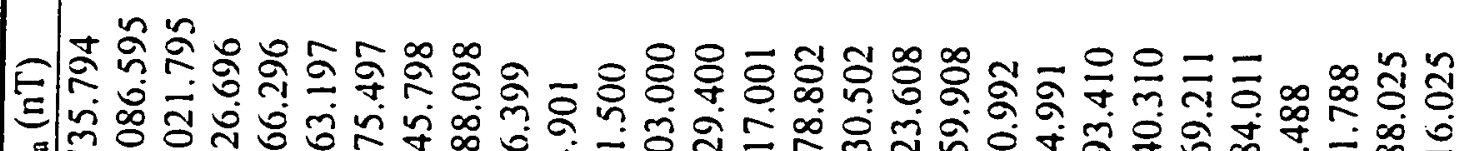
L्य

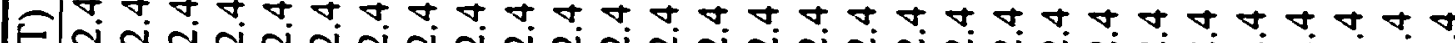

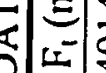

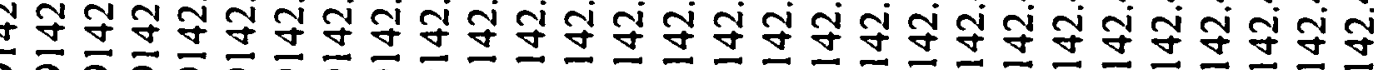

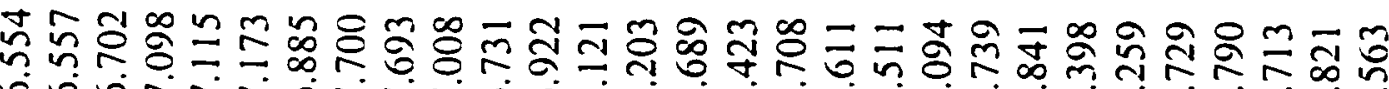

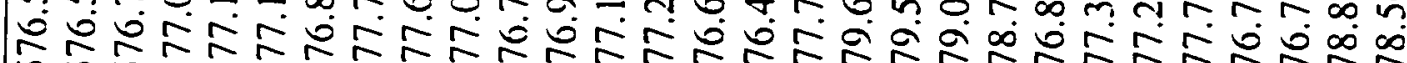

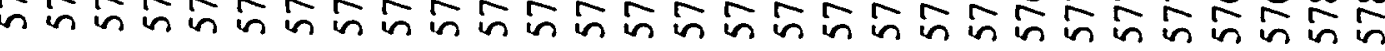
N $N$ N

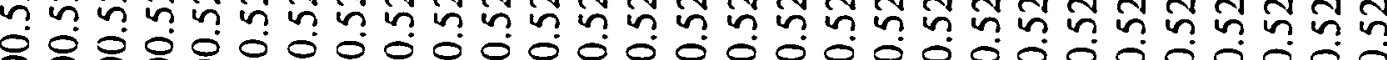

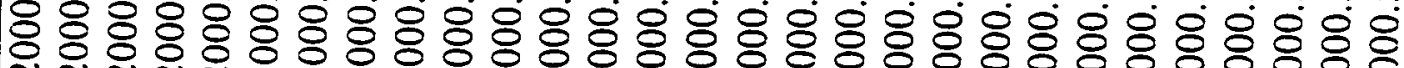

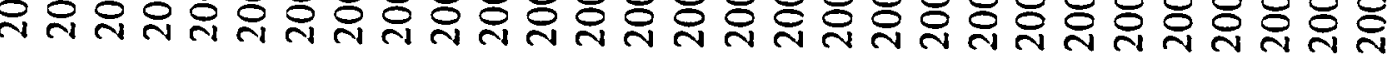

莡

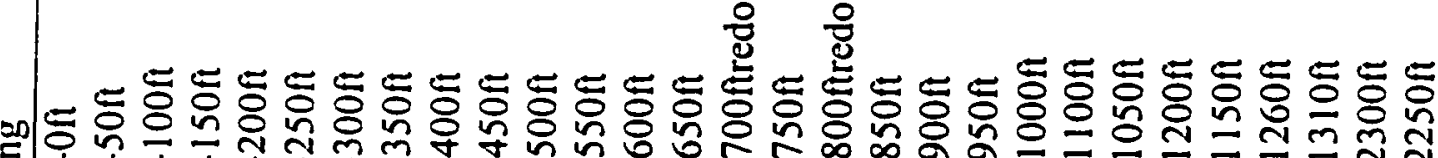

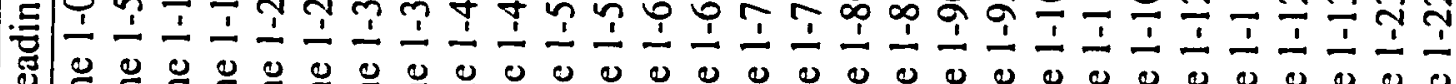

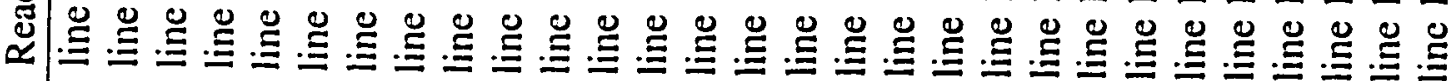


๙ิ๋

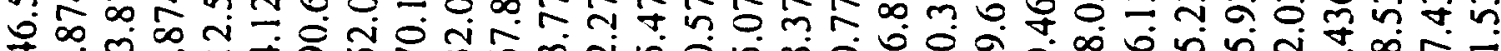

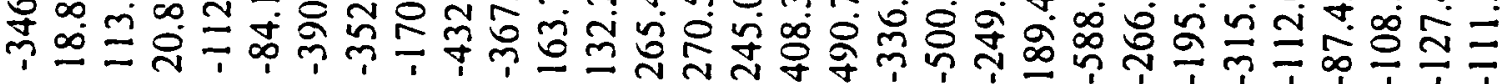

0000000000000000000000000000000 ษษษ

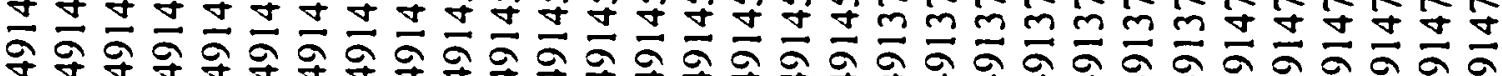

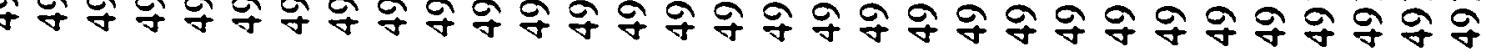

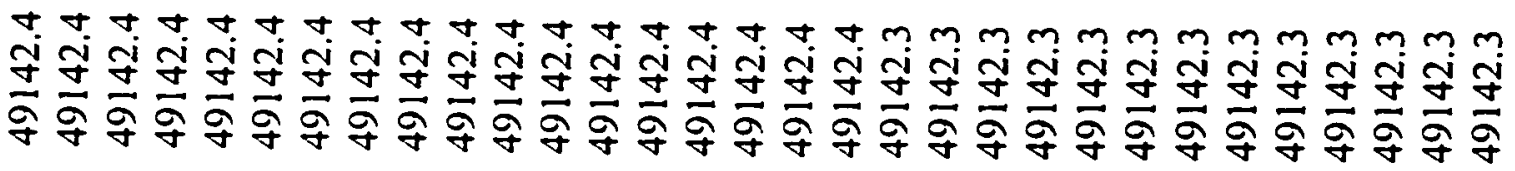

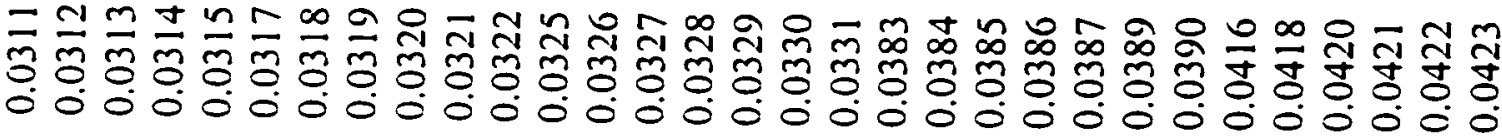

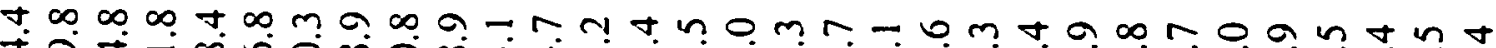

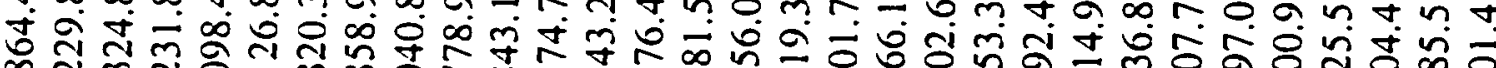

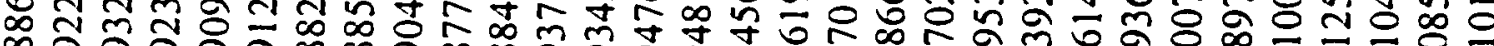

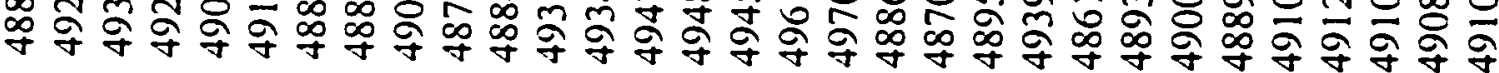

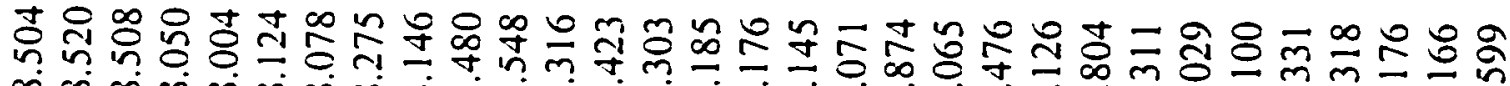

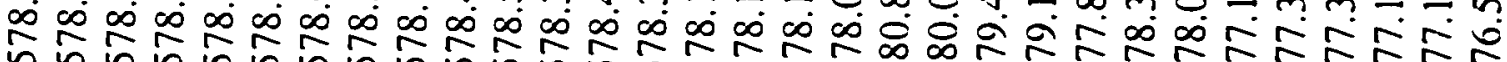

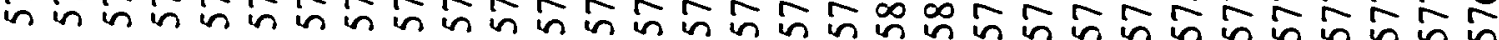

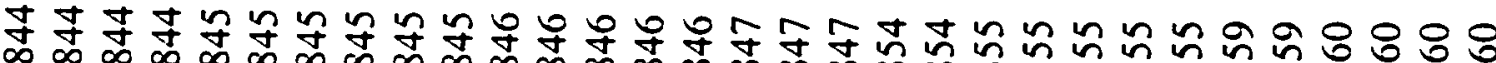

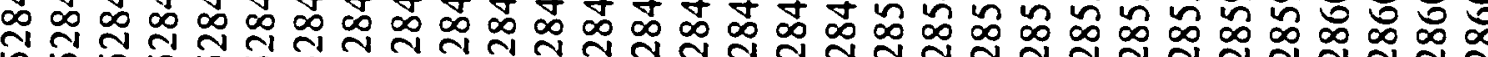

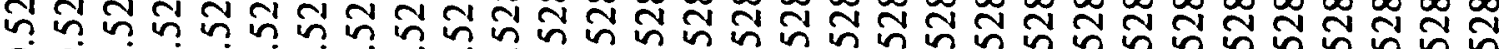

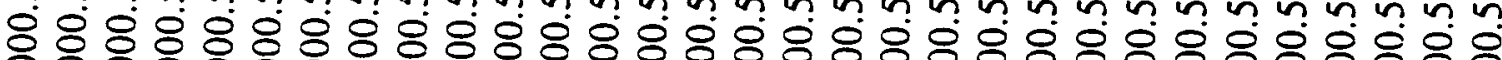

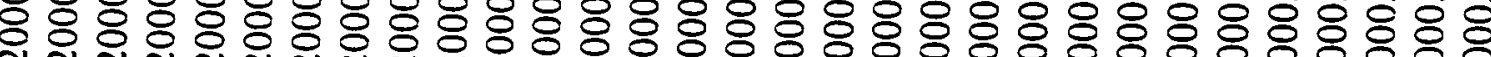

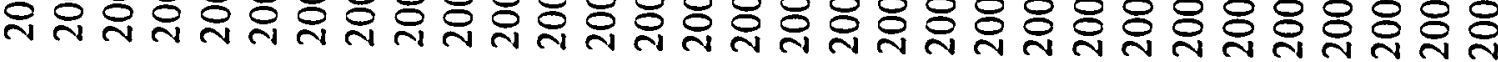

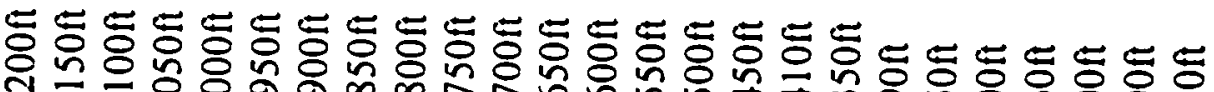

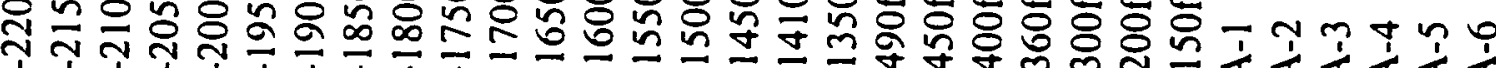

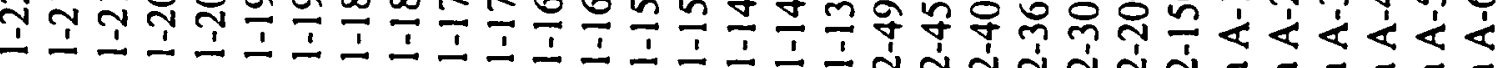

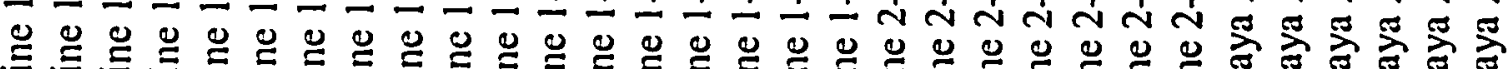

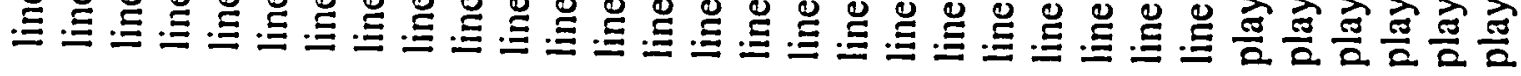




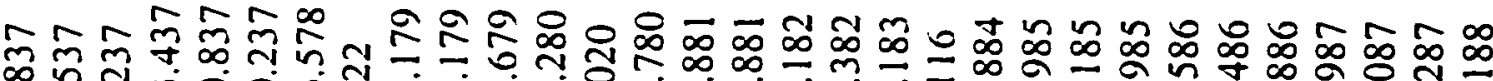

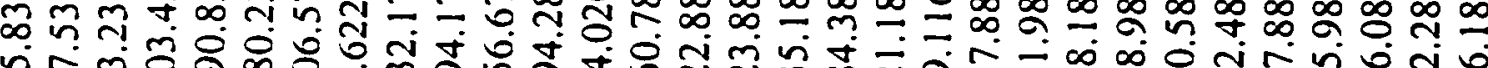

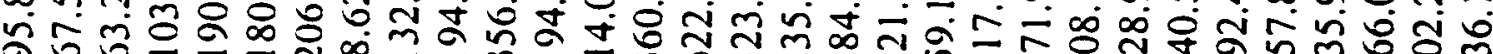

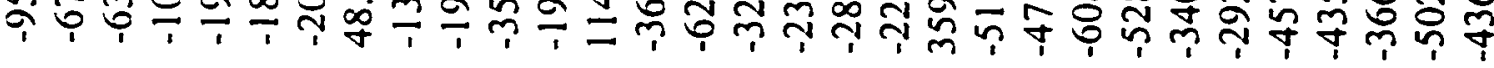

0.000 .00000000000000000000000000

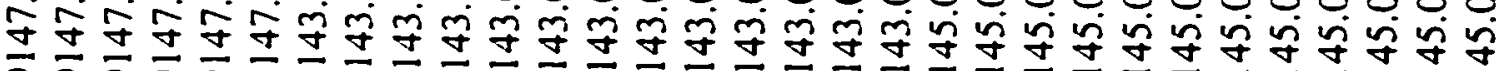

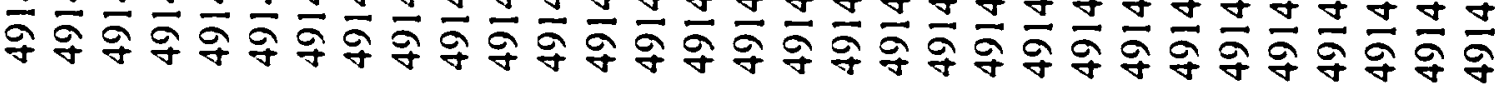

mm ช

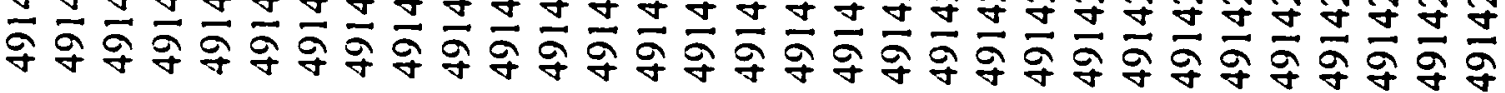

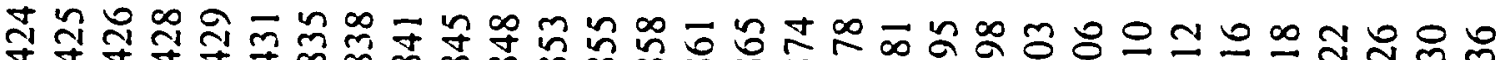

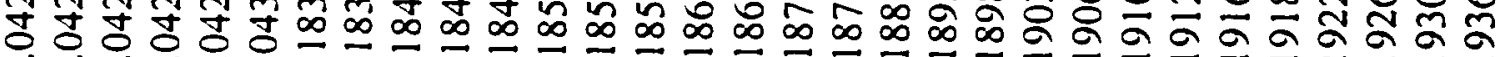

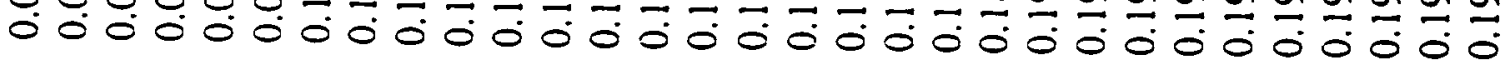

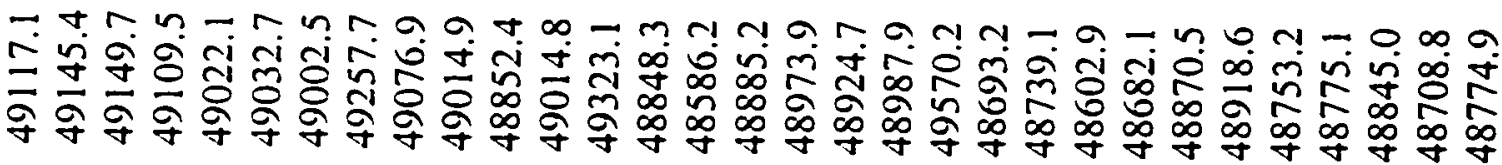

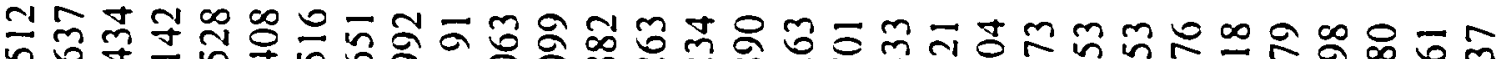
o ju

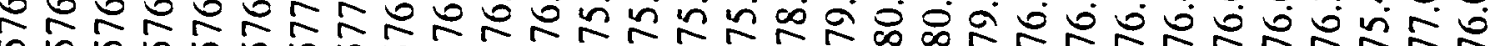

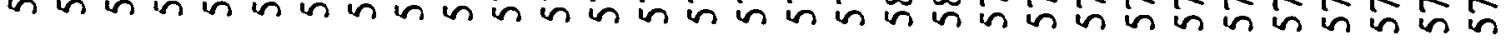

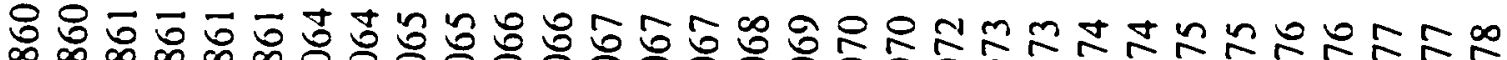

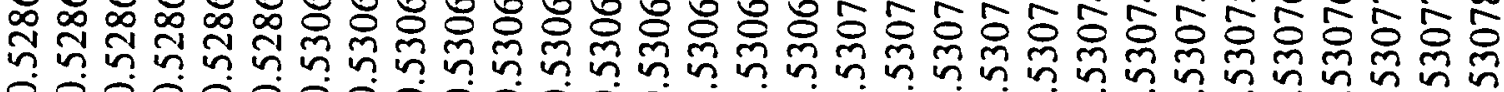

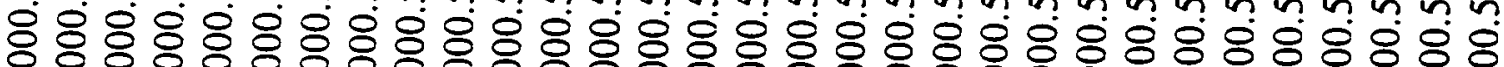

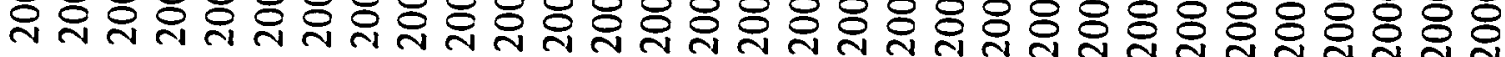

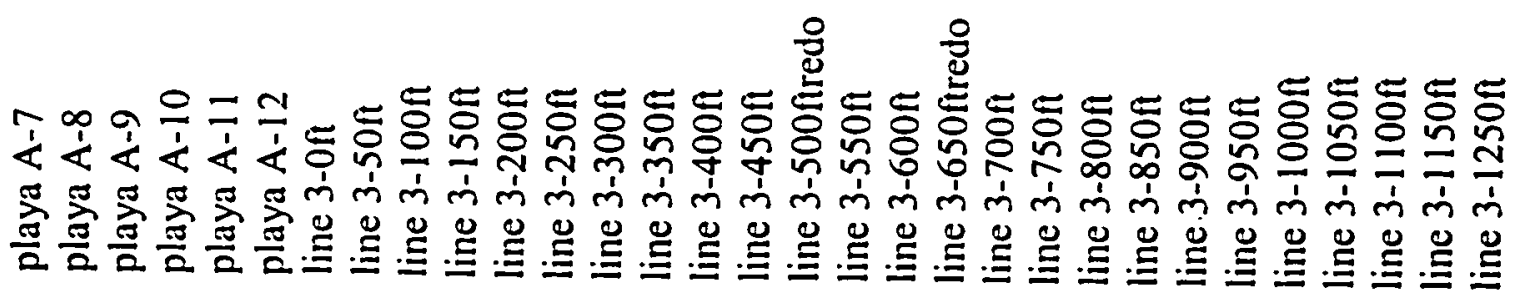




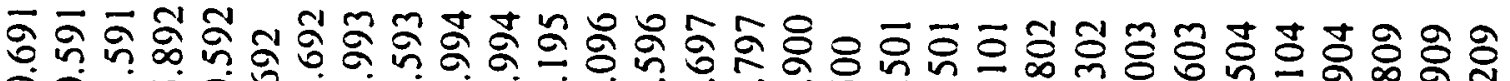

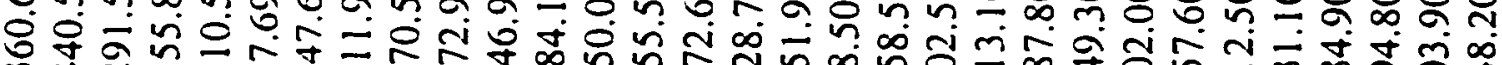

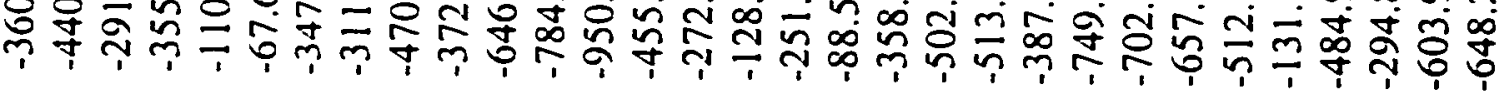

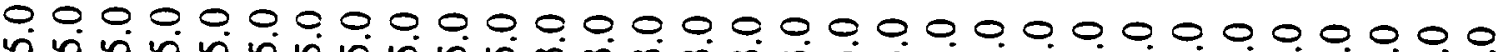

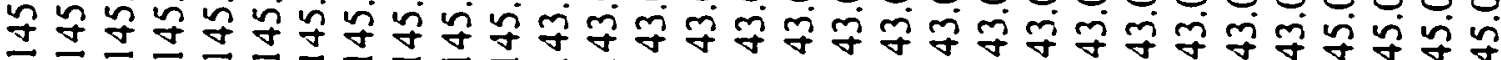

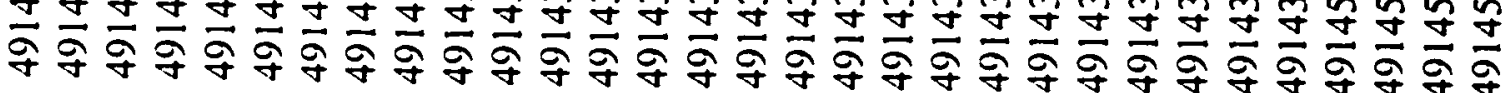

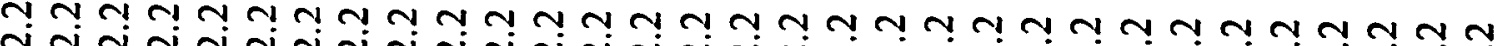

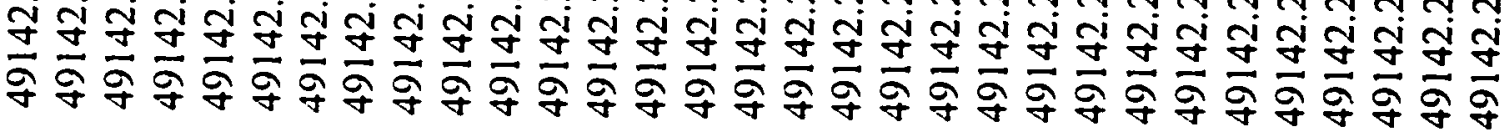

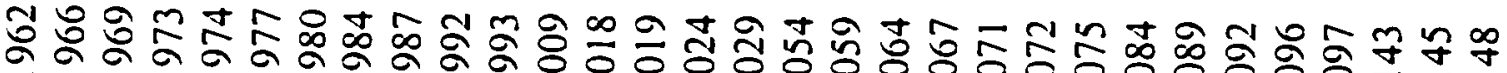
000000

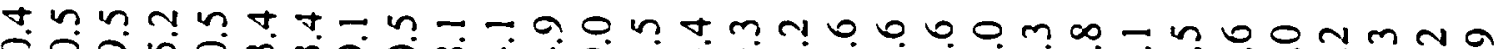

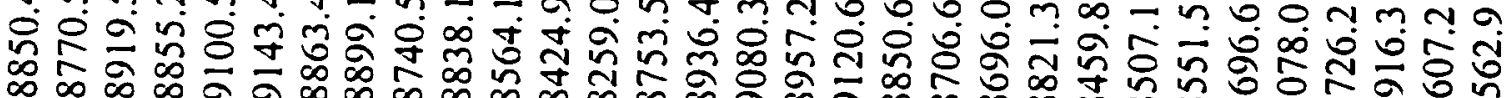

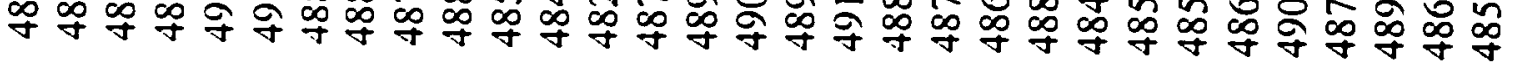

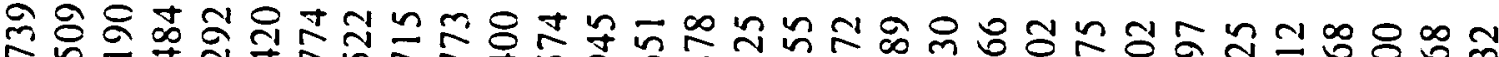

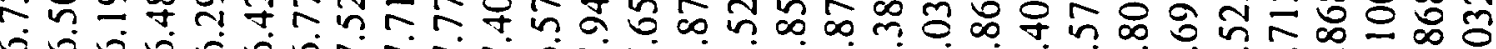
200

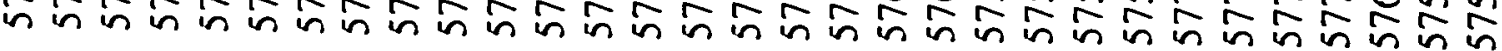

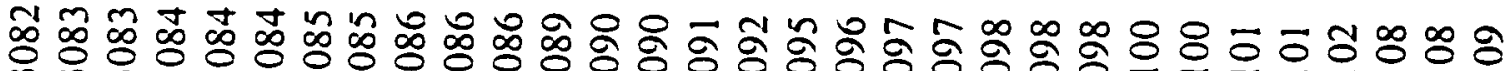

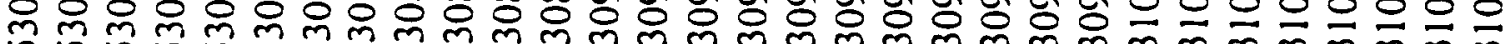

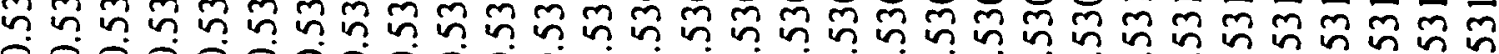

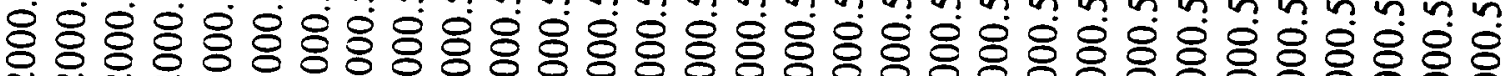
霖

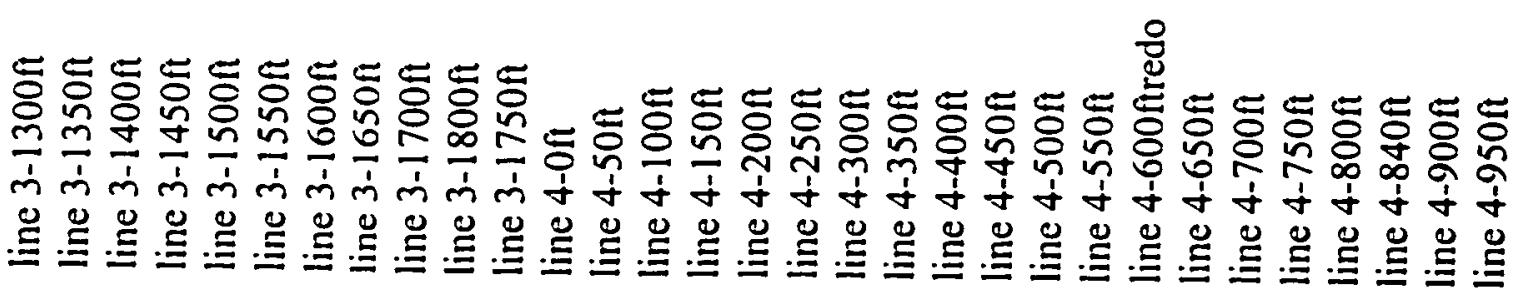




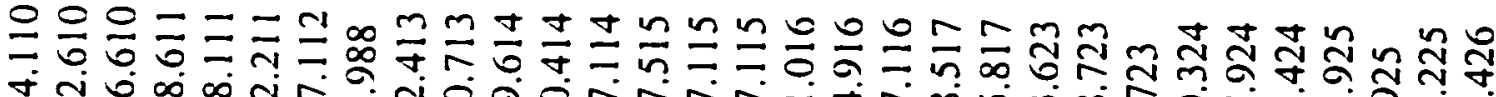

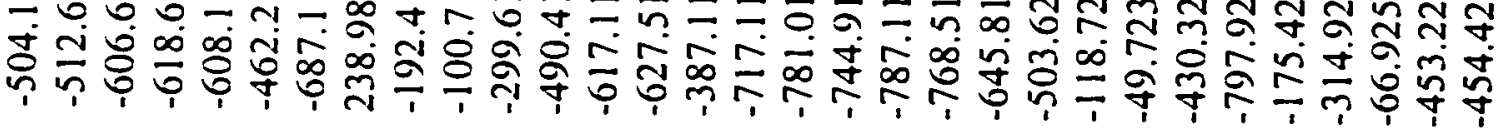

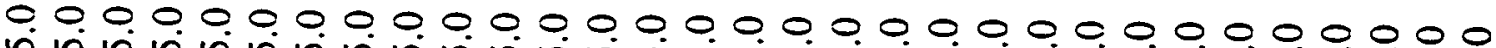

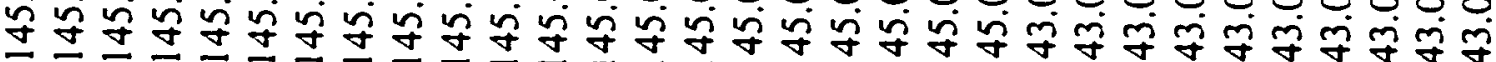

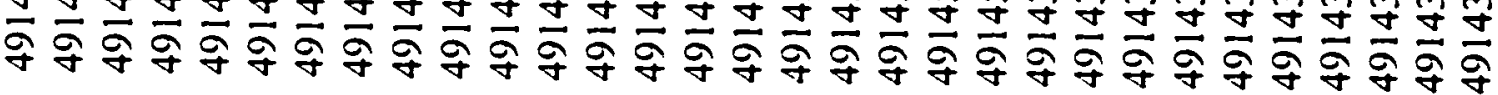

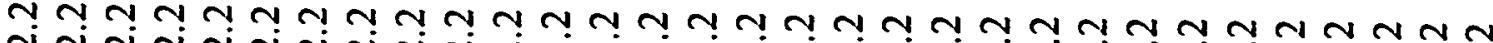

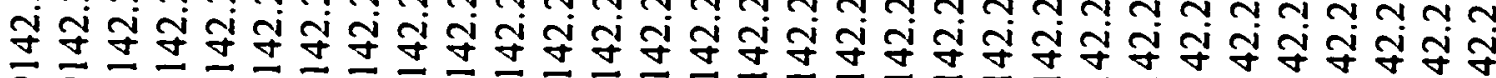

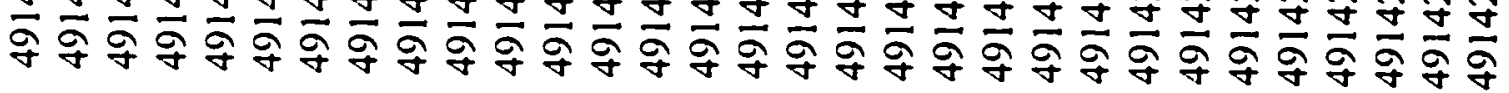

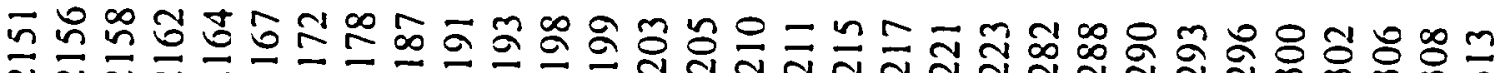

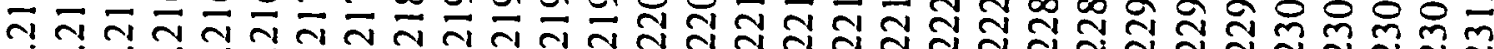

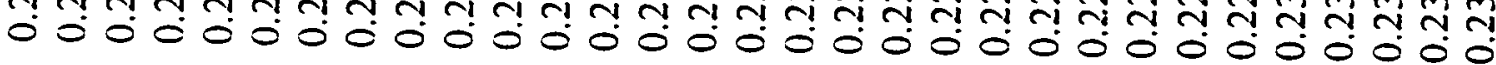

onnmo a o r t t n 仓்

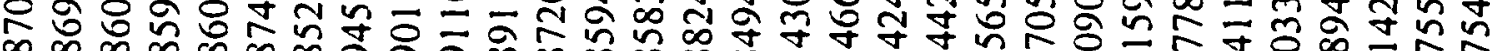

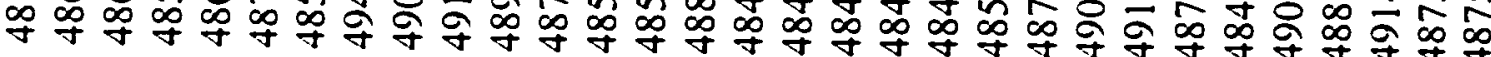

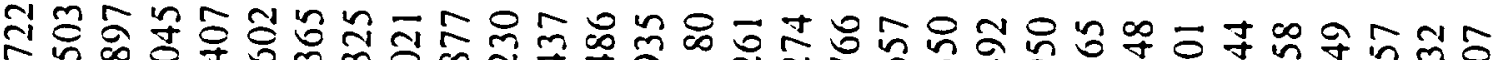

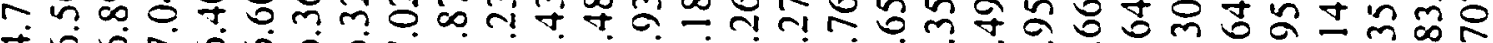

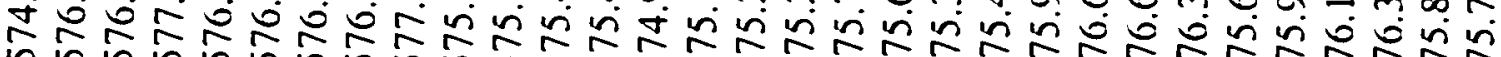

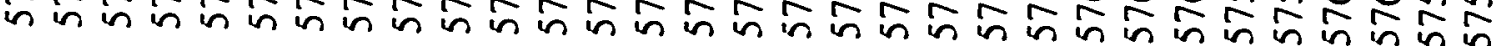

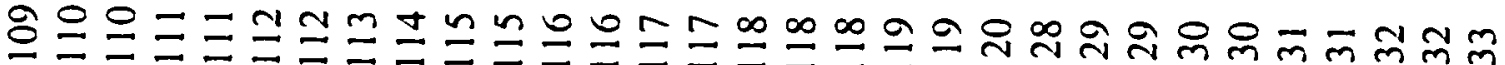

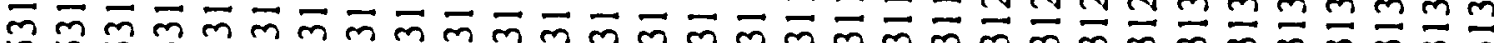

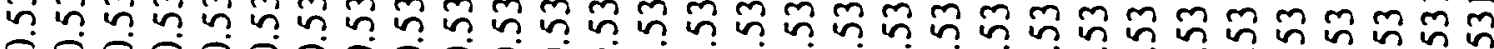

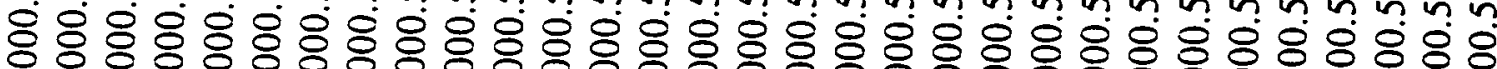

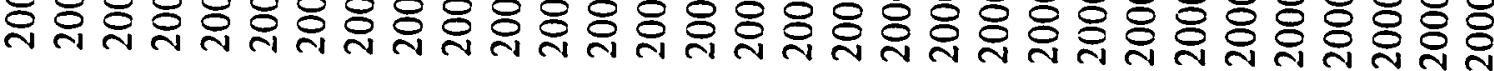

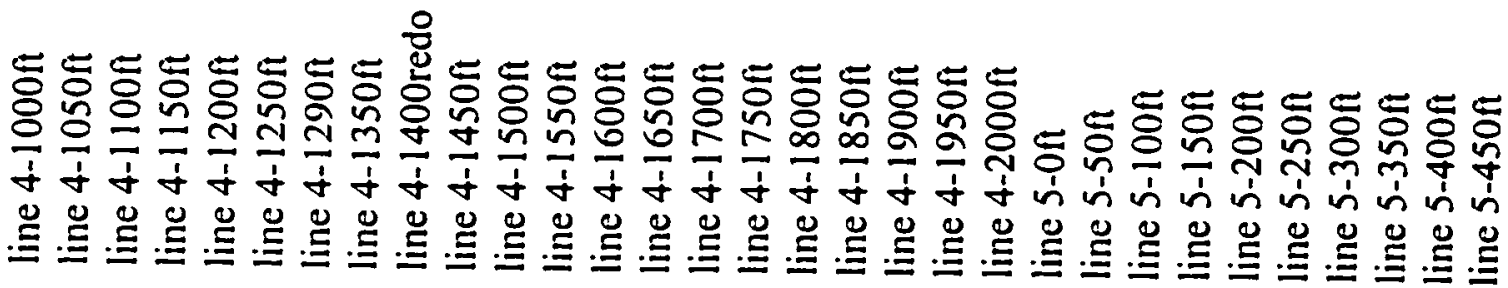




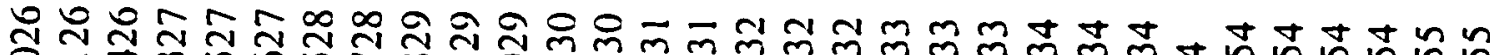

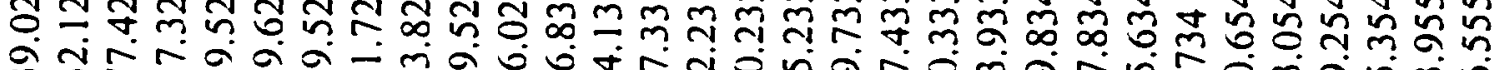

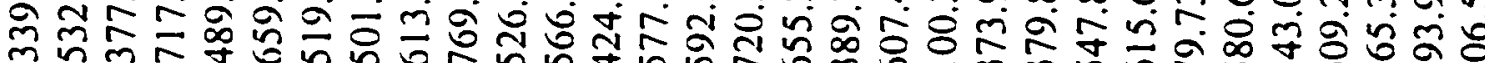
ๆิ

0000000000000000000000000000000

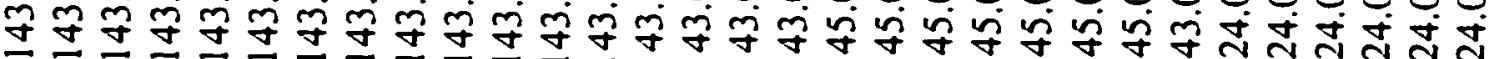

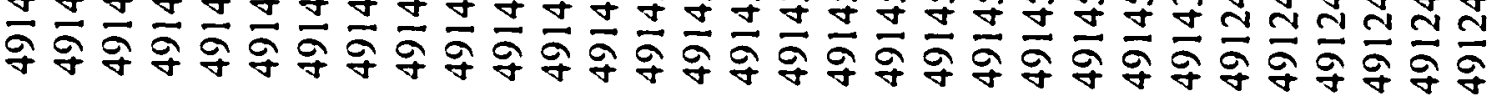

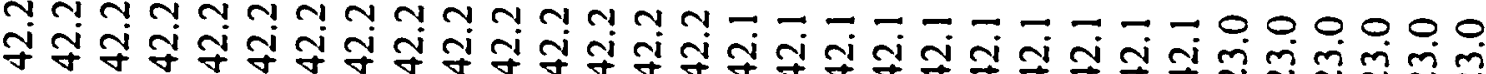

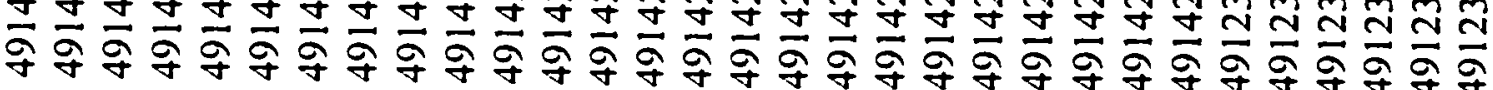

므슬

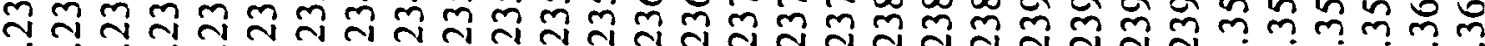

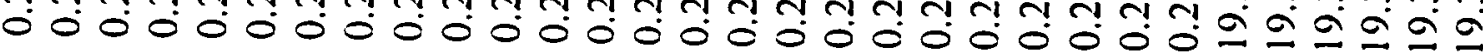

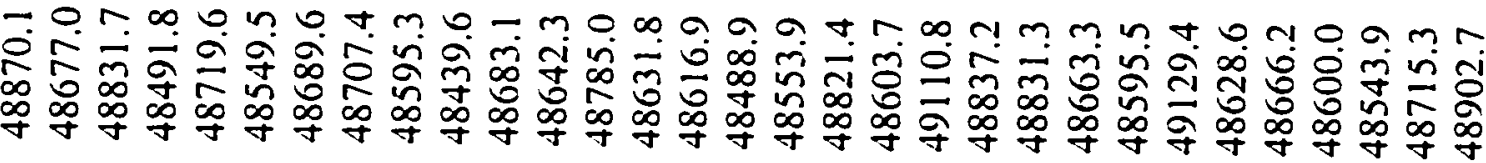

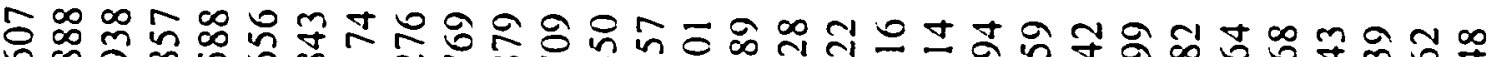
ర一

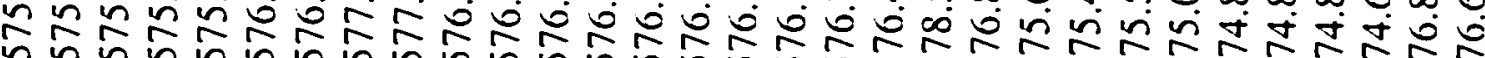

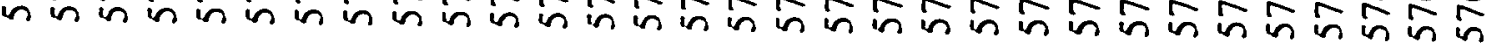



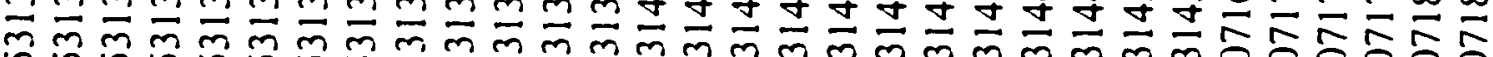

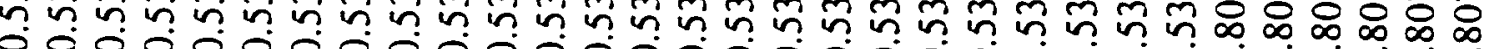

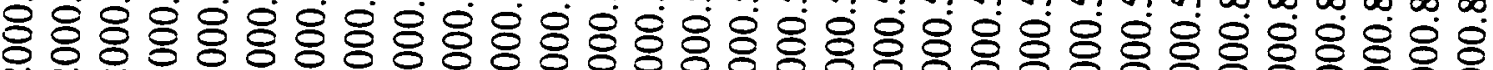

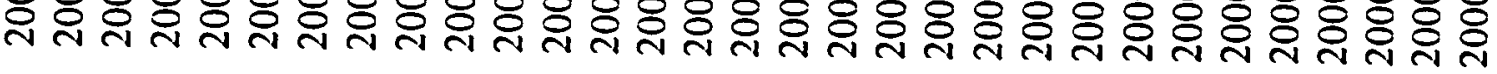

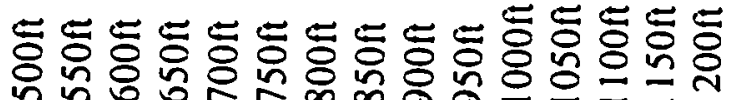

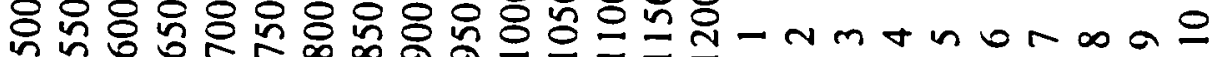
n่ n่

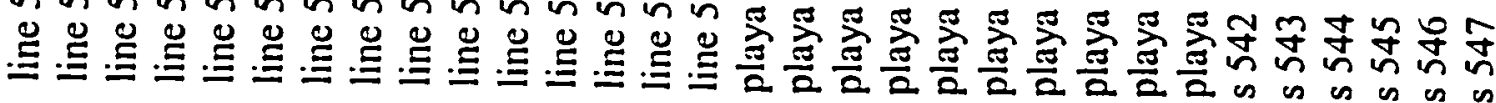




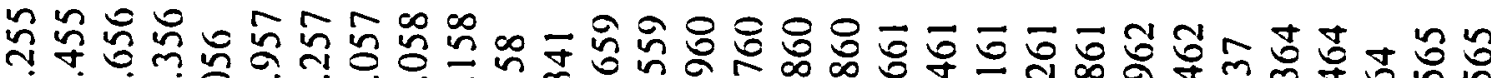

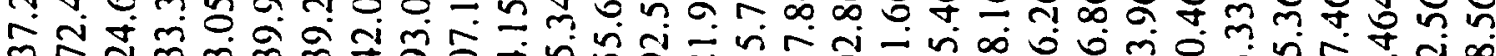

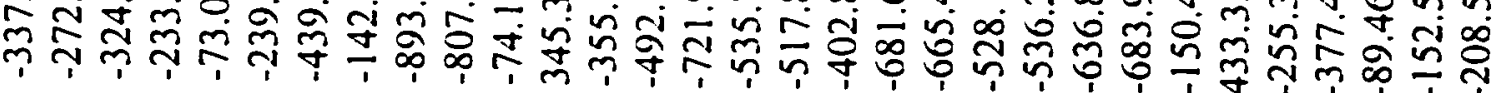

0000000000000000000000000000000

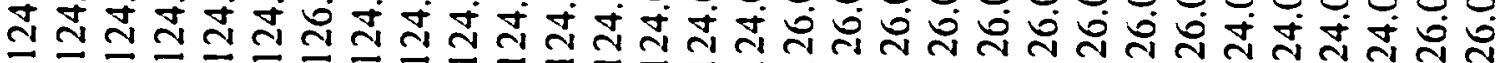

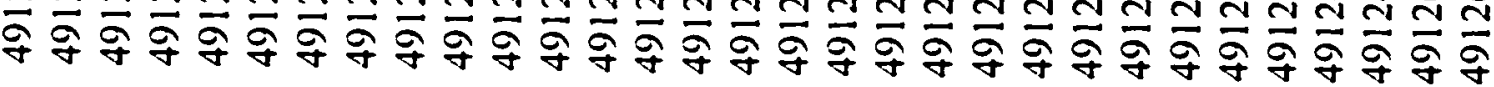

0000000000000000000000000000000 ขิ ને

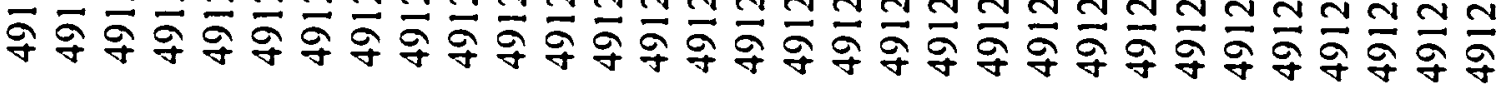

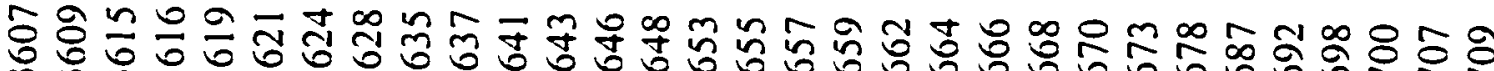

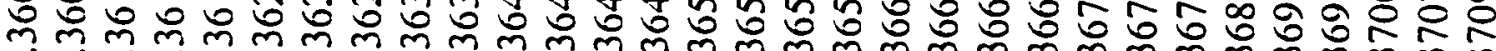

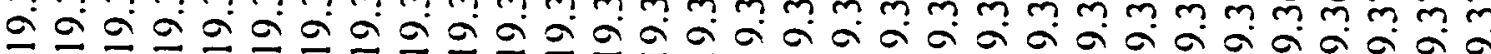

0 o 0 ,

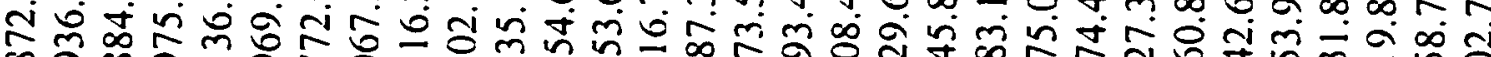

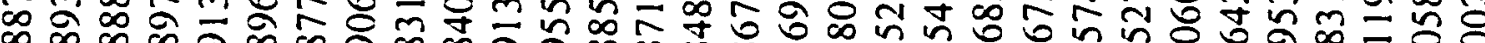

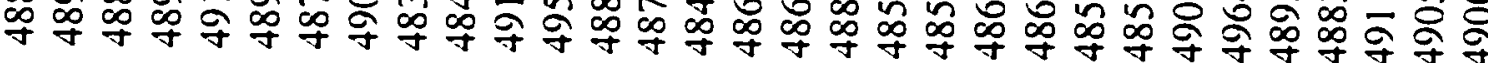

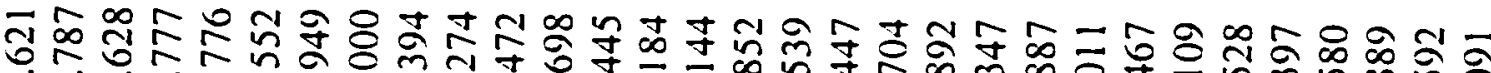

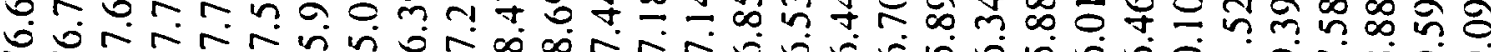

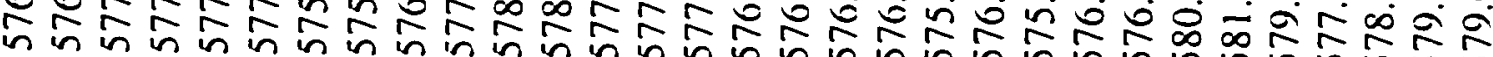

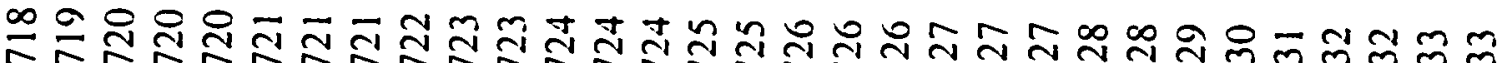

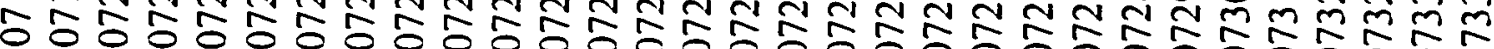
$\infty \infty \infty \infty \infty) \infty \infty \infty \infty \infty \infty \infty \infty) \infty \infty$ 穴完

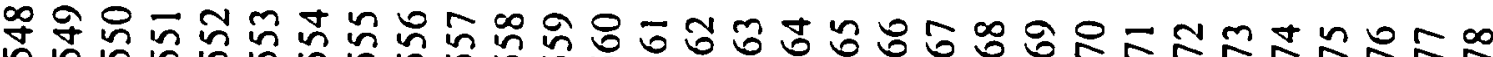

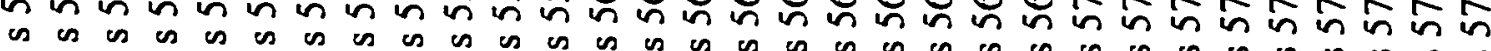




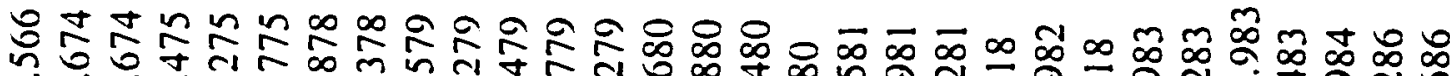

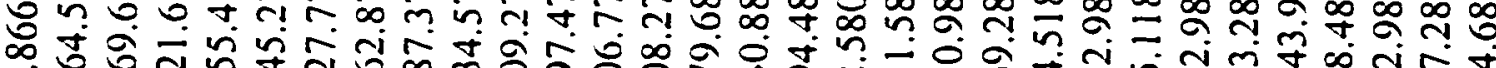

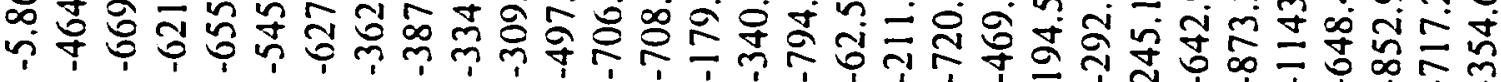

0000000000000000000000000000000

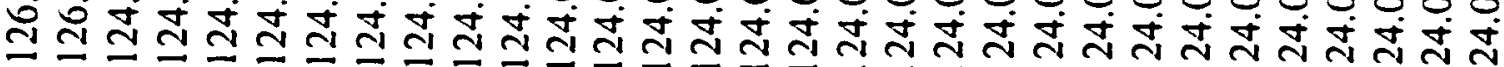

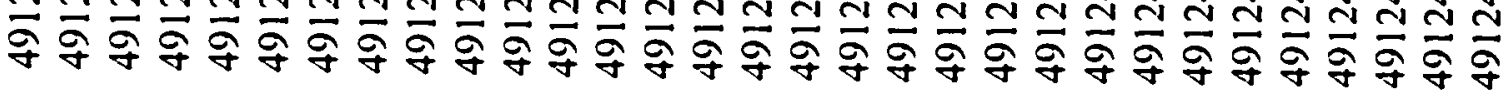

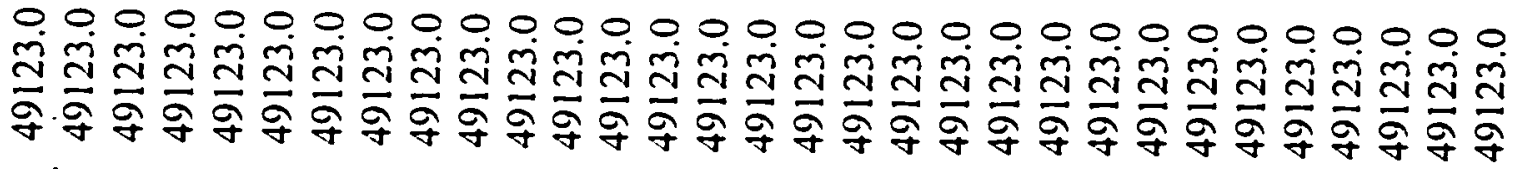

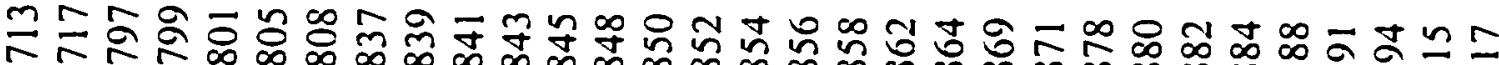

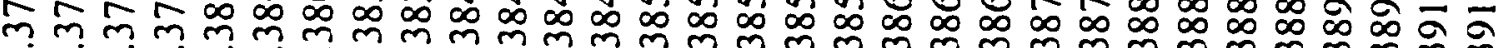

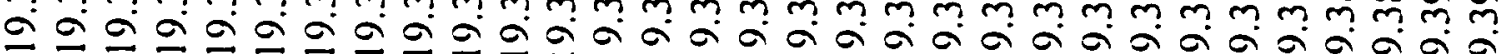

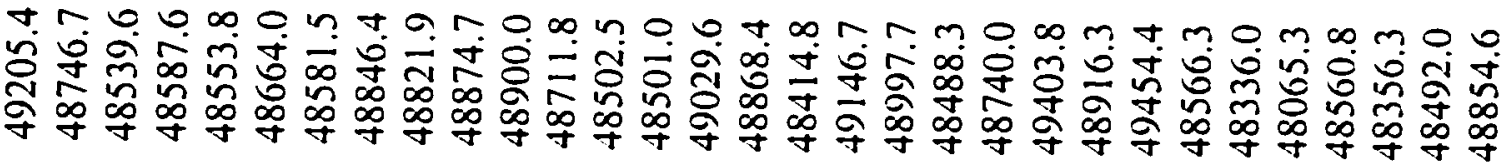

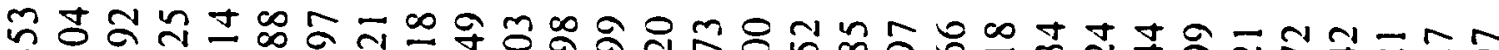

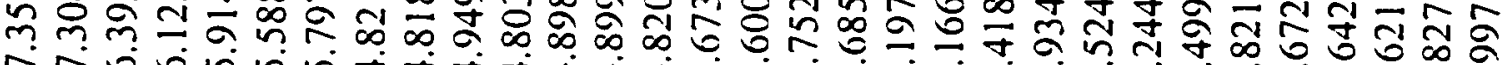

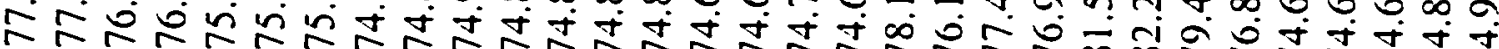

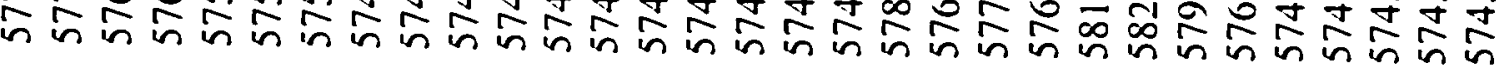

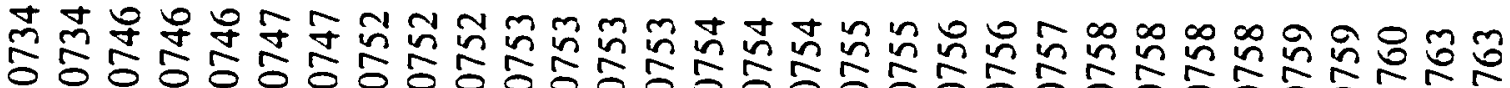
$\infty \infty \infty \infty \infty \infty \infty \infty \infty \infty \infty \infty \infty) \infty \infty$

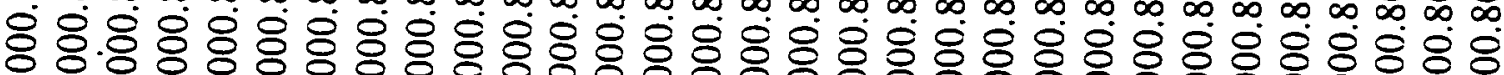

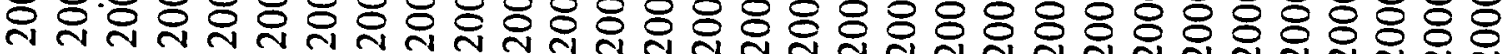

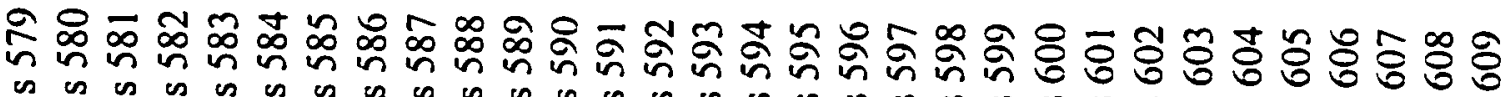


同

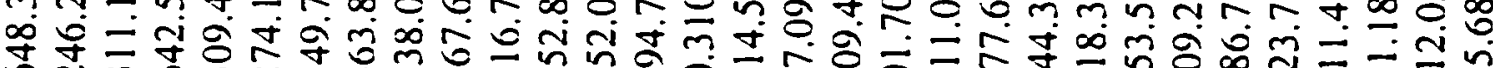

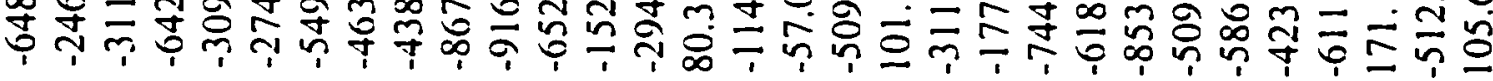

0000000000000000000000000000000

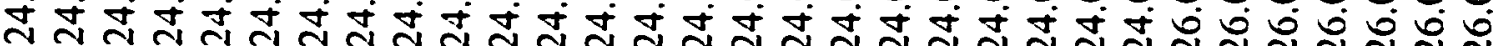

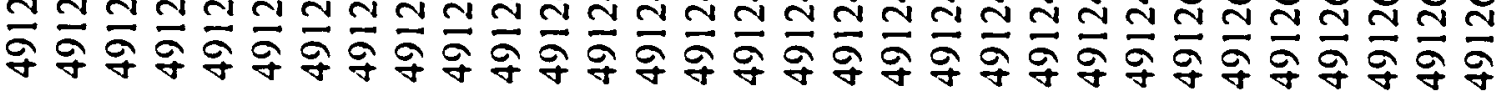

$0.00000000000000000000000009 a 9 a$

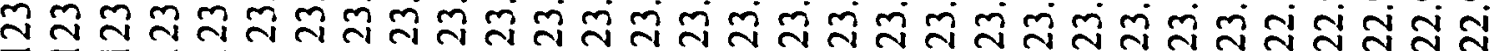

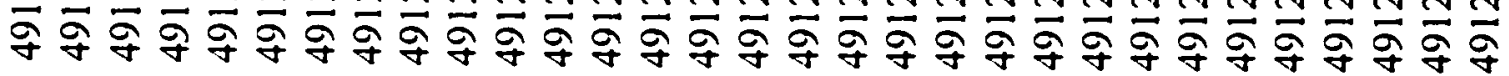

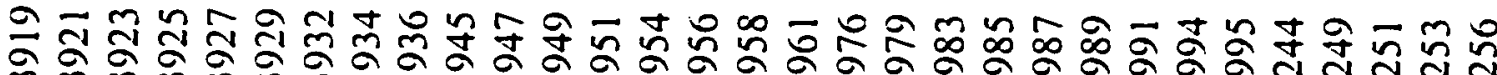
సิ

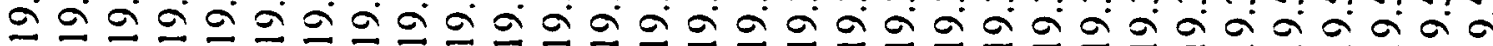

a ஜ்

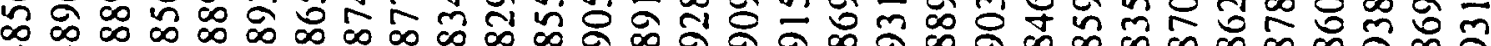

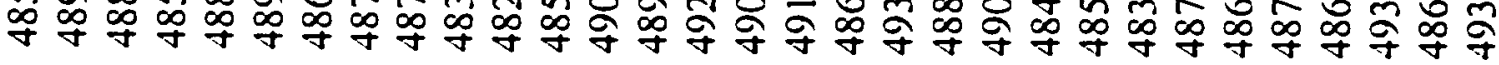

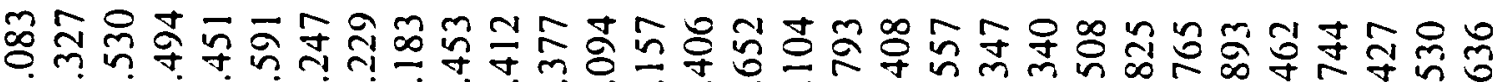

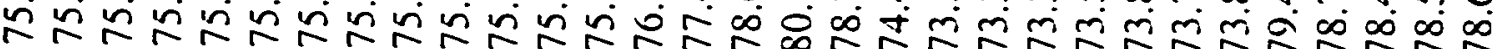

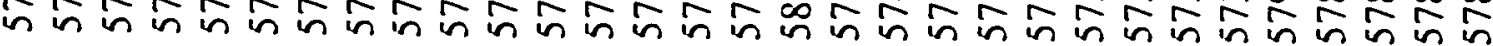

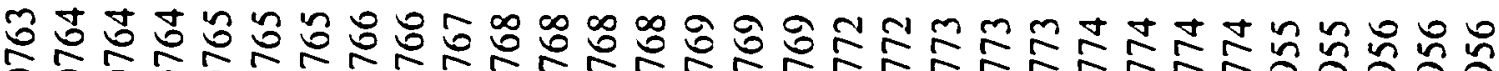
5ร5 5555555

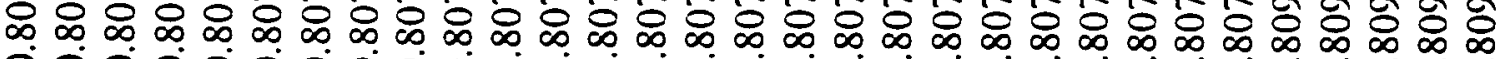
ठ்

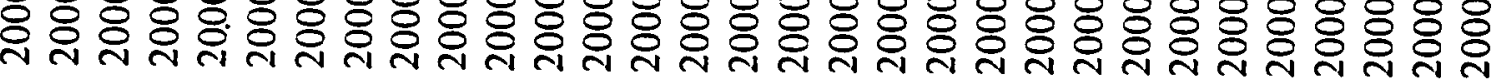

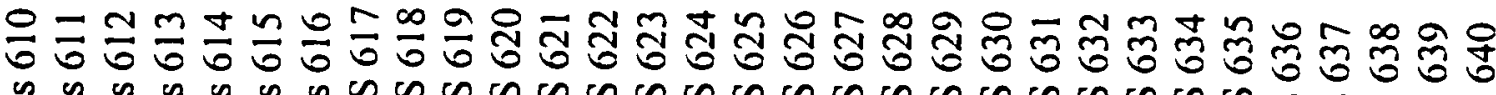

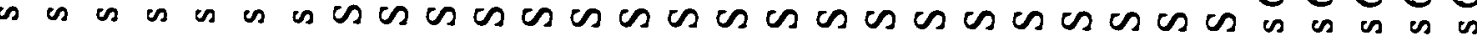



光

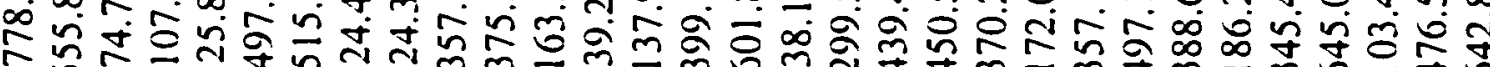

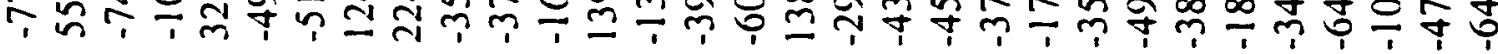

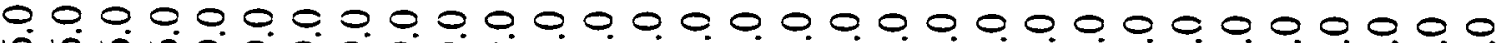
ن i i

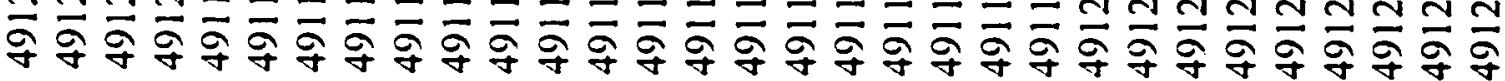

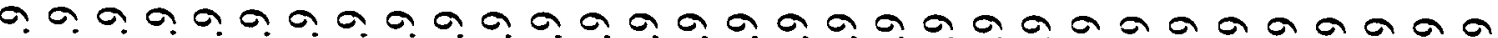

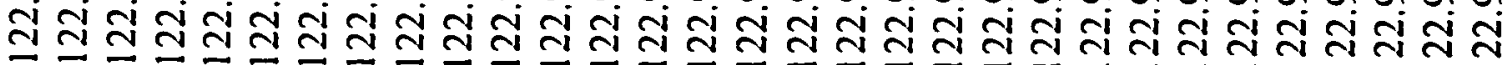

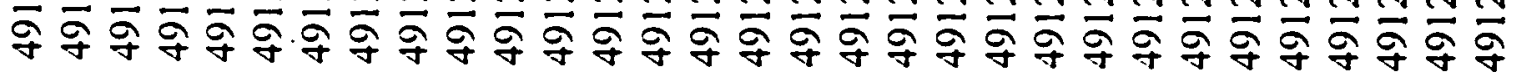

車 잉

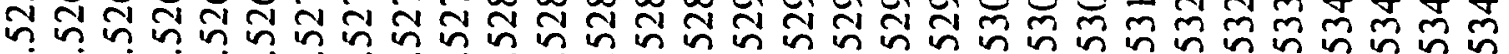

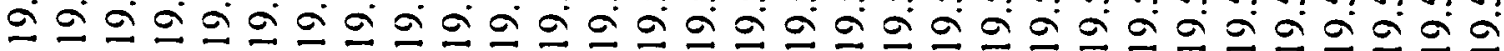

-mก

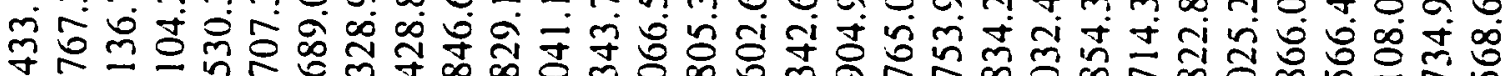

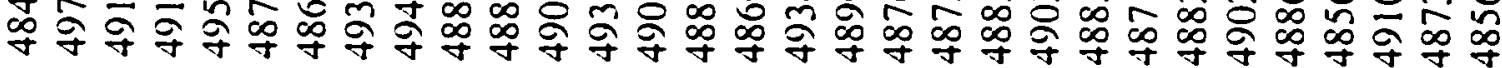

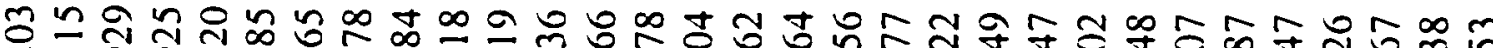

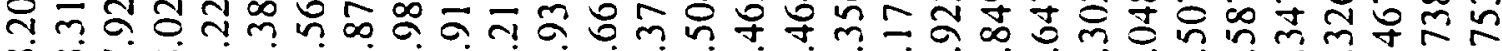

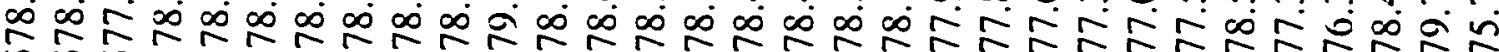

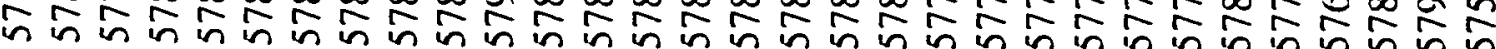

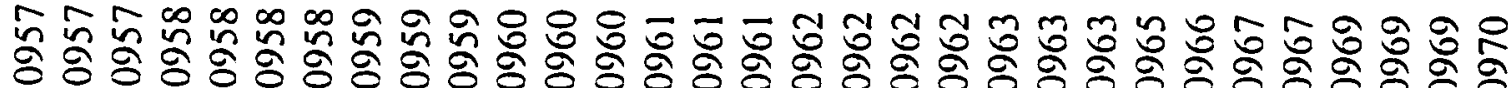

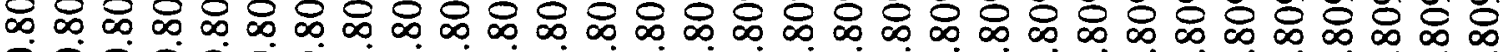
ठ்

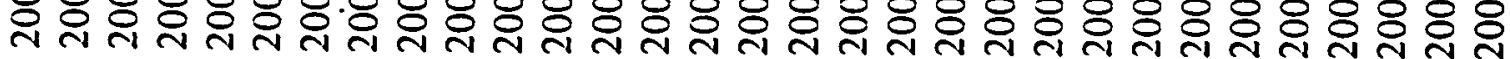

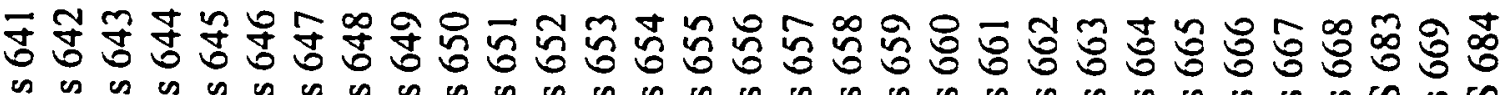




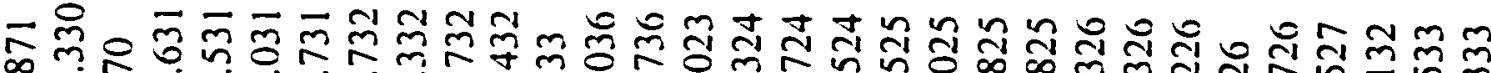

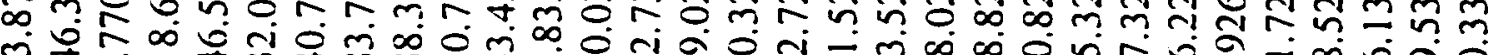

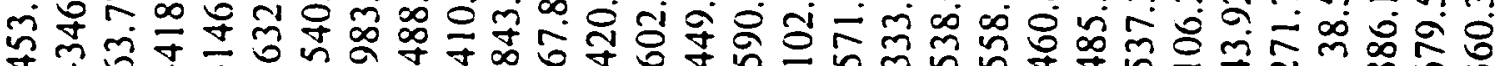
व

0.00000000000000000000000000000

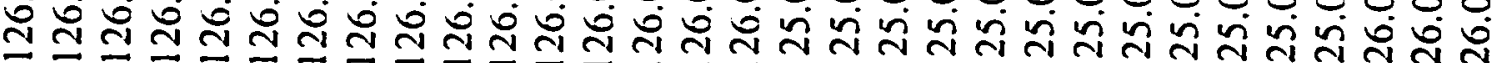

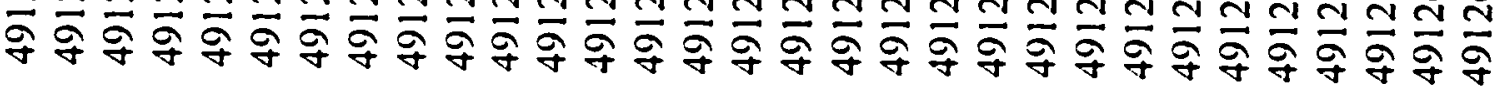

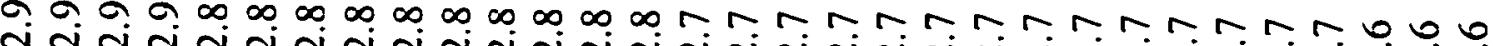
สิ สิ

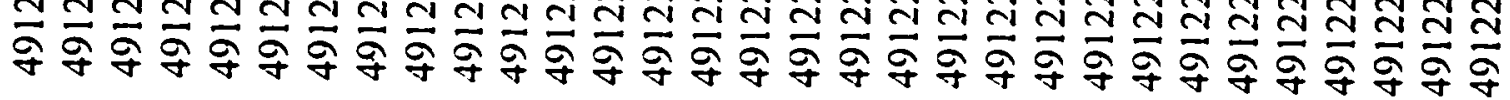

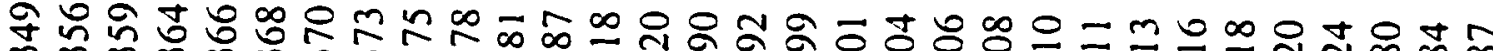

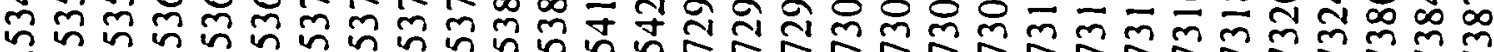

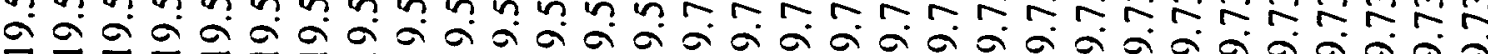

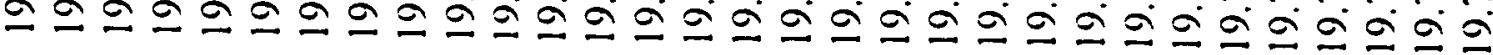

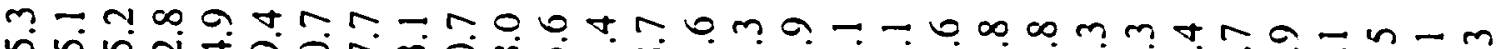

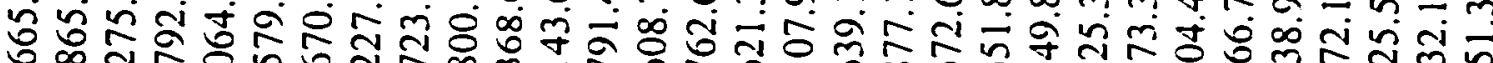

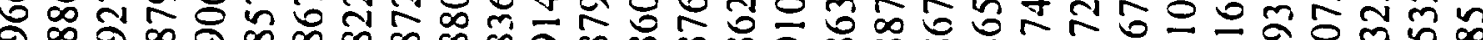

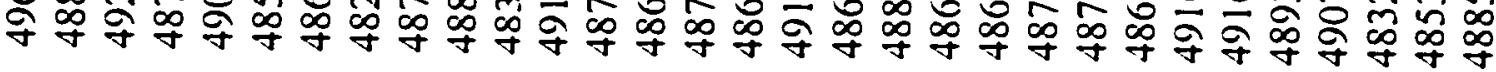

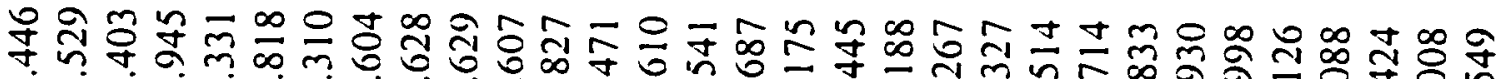

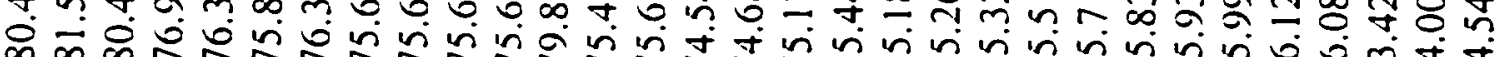

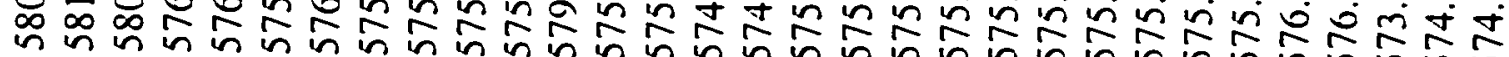

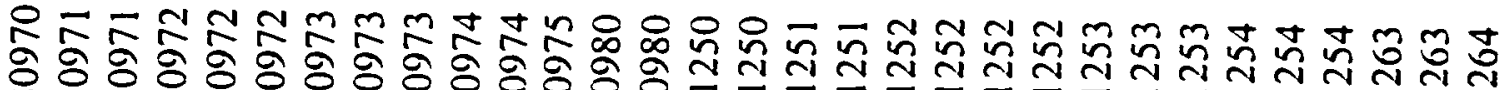

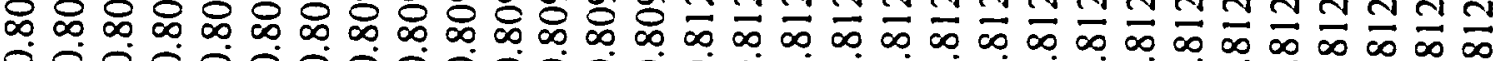
ठ் ठ்

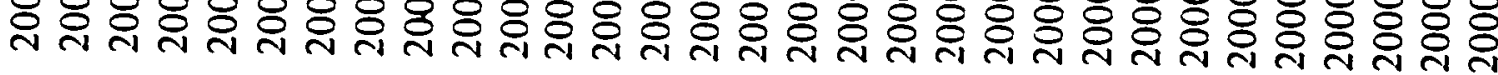

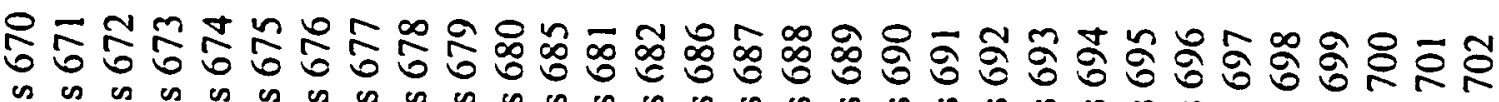

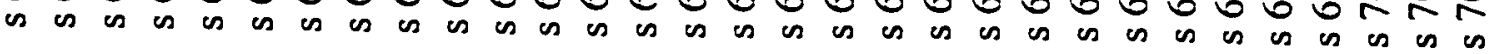




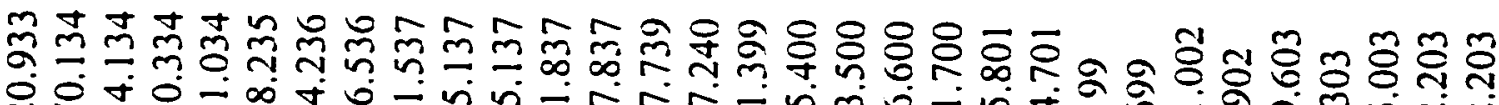

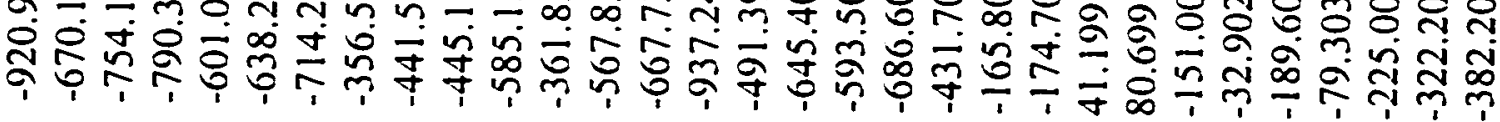

0.00000000000000000000000000000

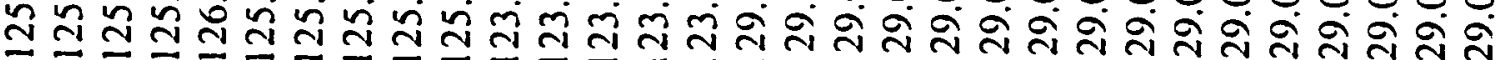

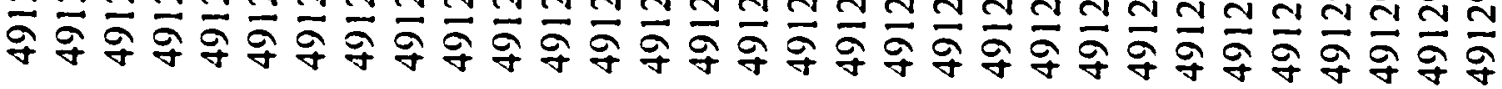

0.

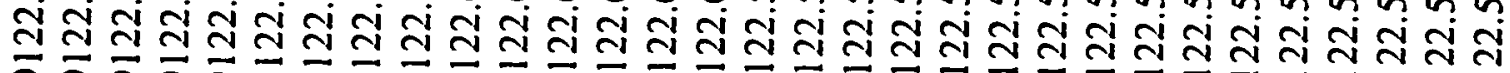

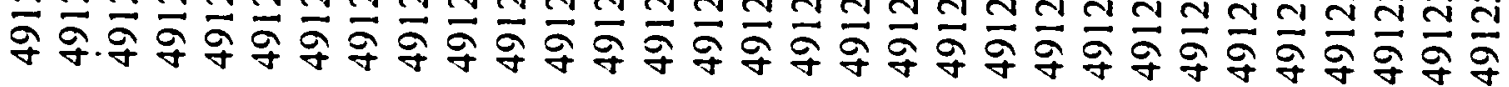

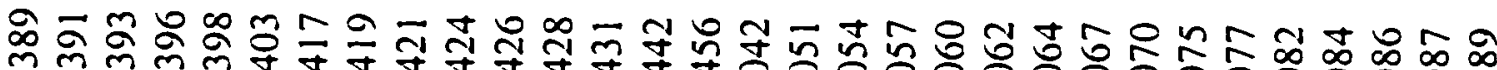

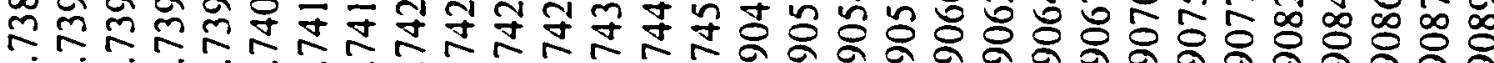
åa

กnnmottकें

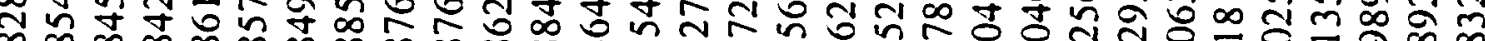

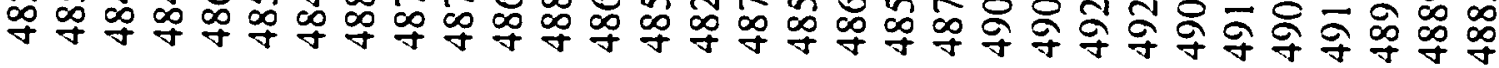

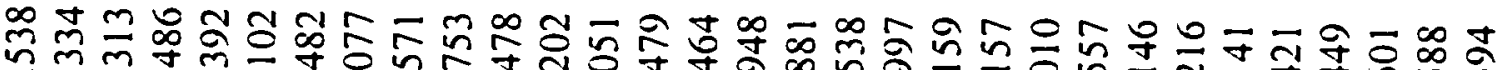

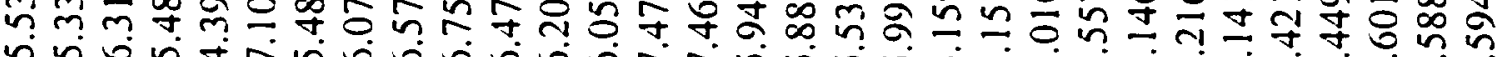

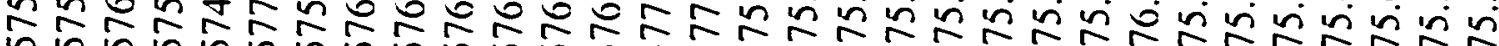

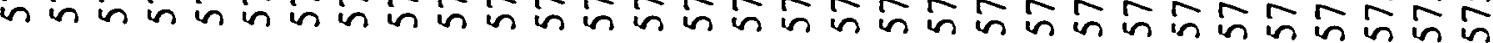

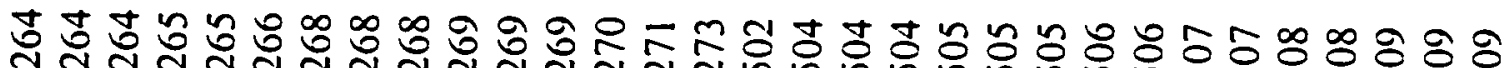

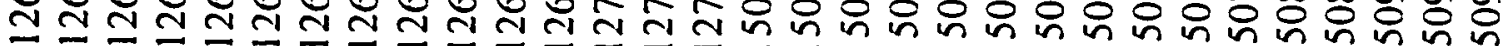

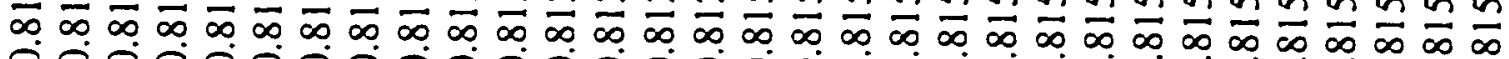
ठ்

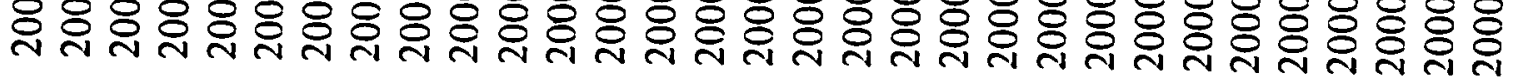

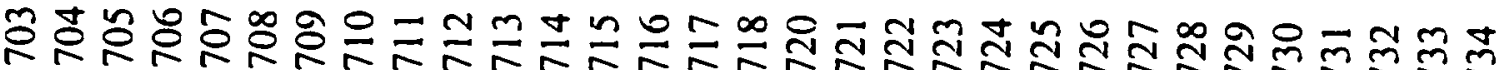

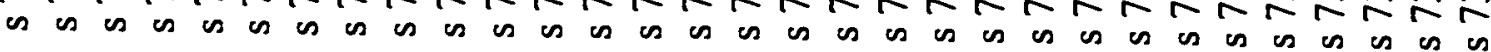




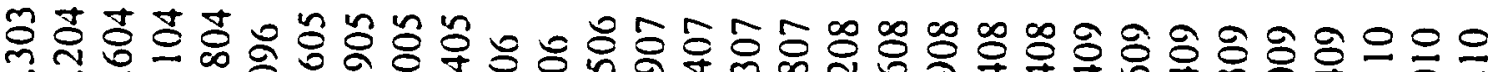

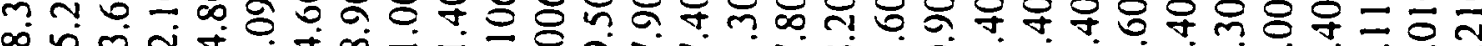

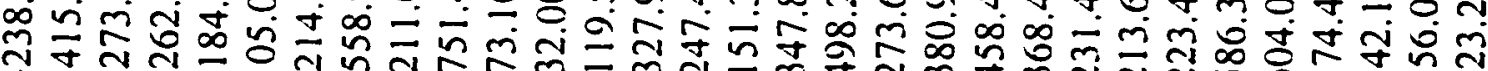

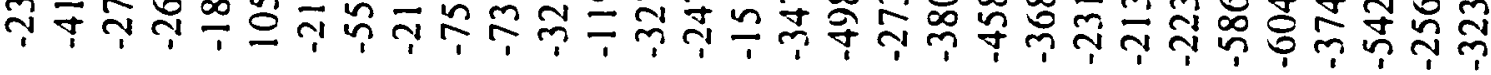

0000000000000000000000000000000

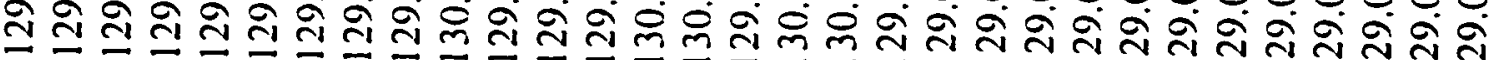

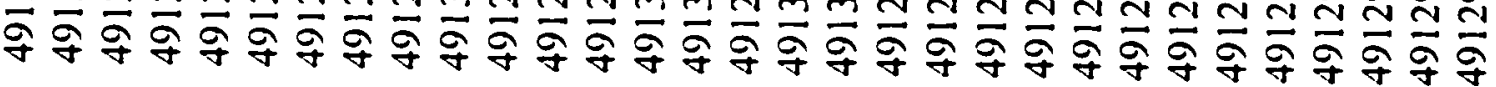

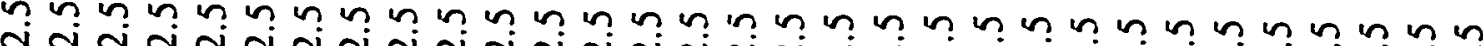

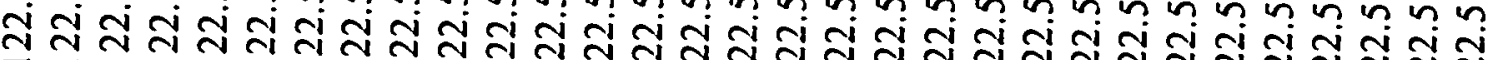

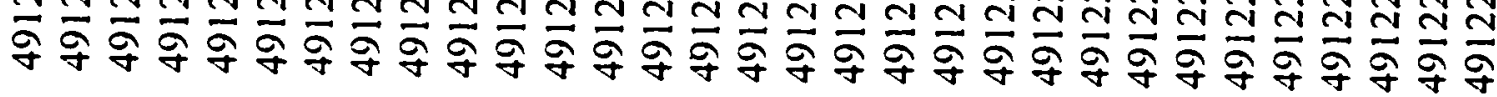

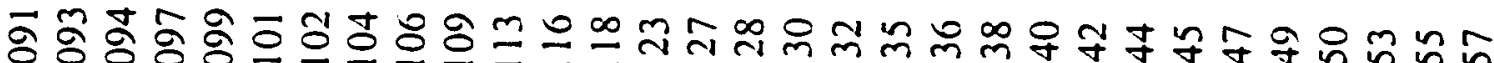

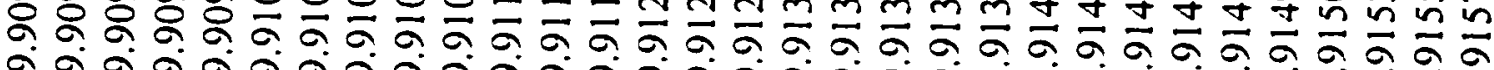

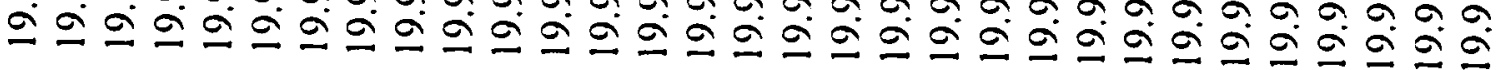

nต

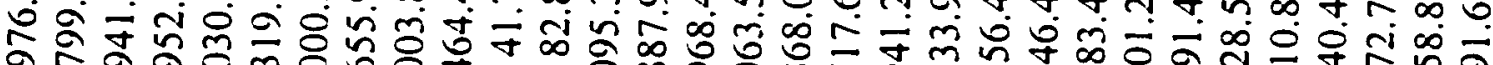

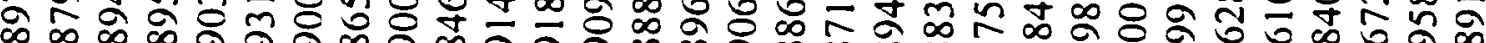

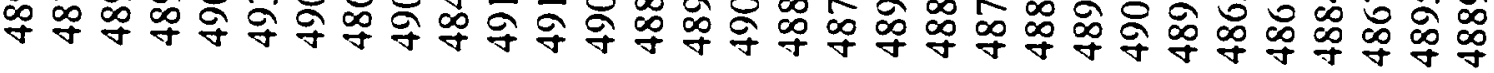

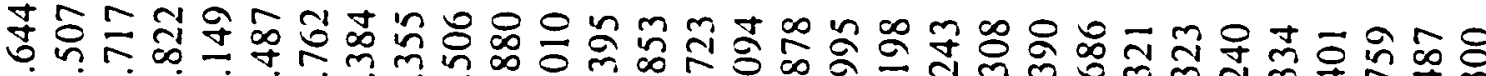

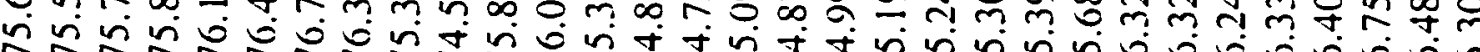

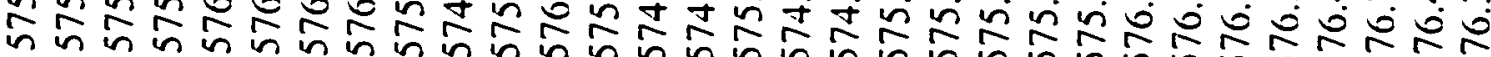

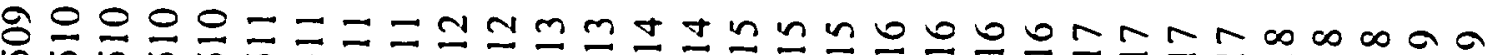

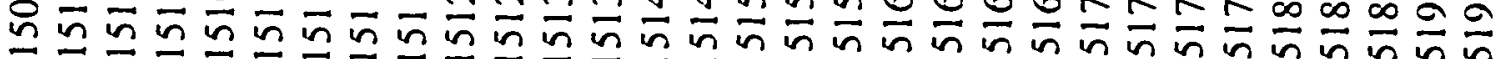

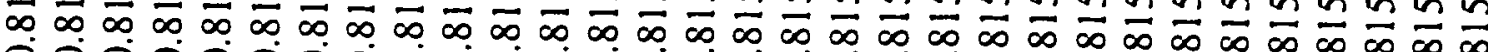
ஓं

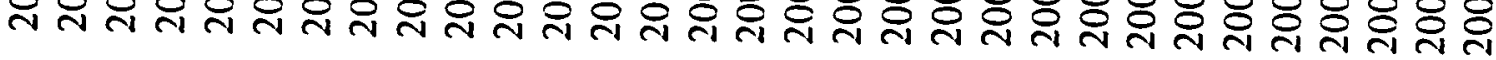

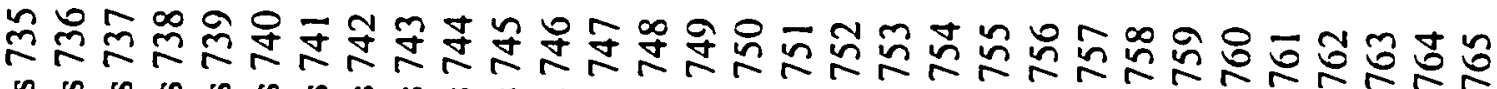

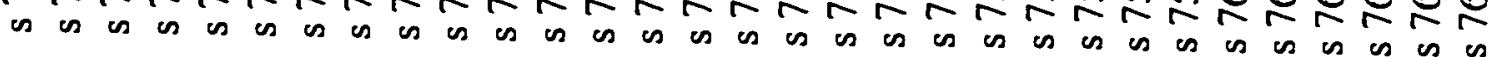




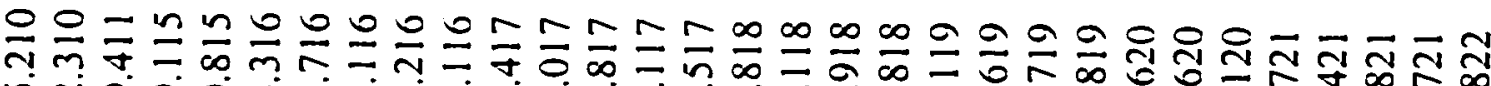
กั กิ่ 귀ำ

0000000000000000000000000000000

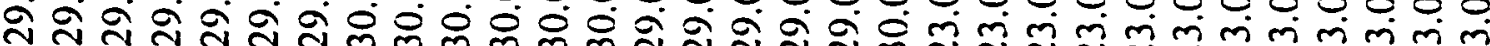

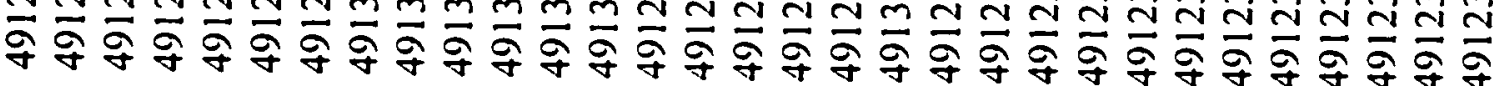

nn

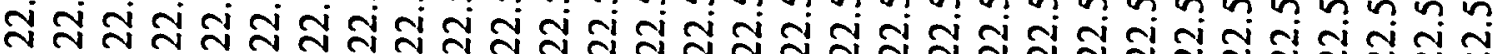

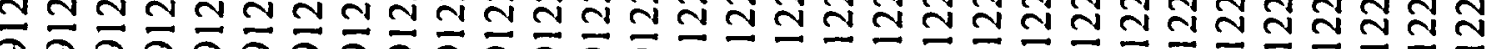

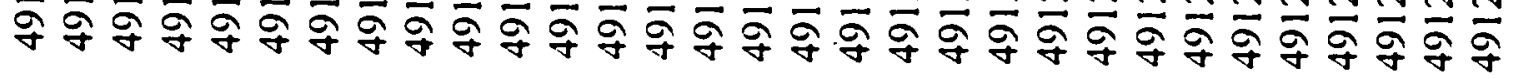

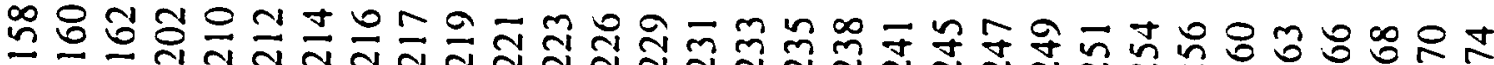

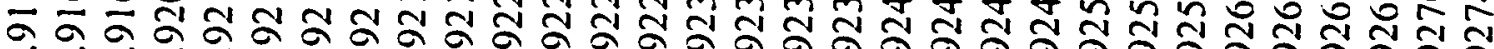

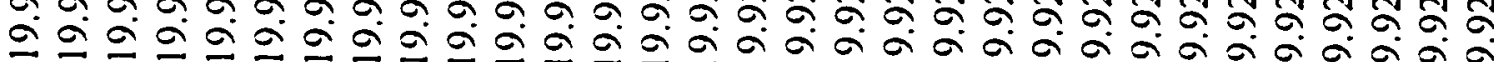

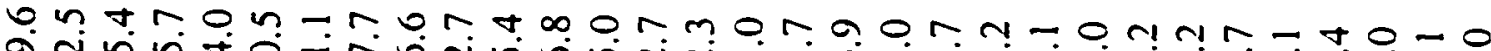
จิ่ป்

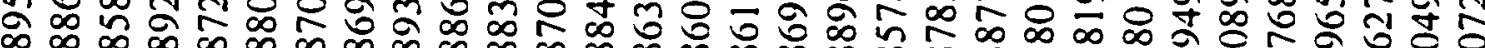

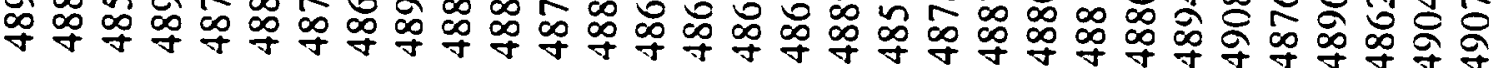

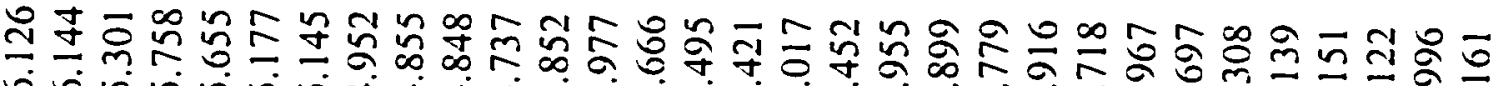

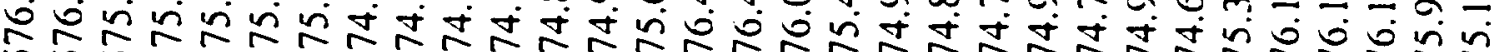

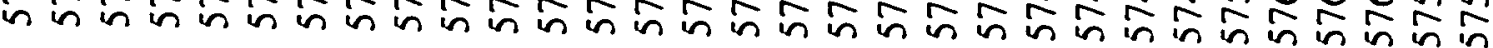

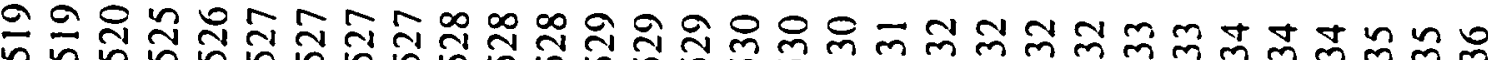
๓n

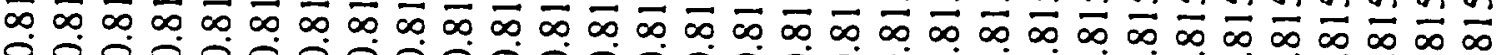

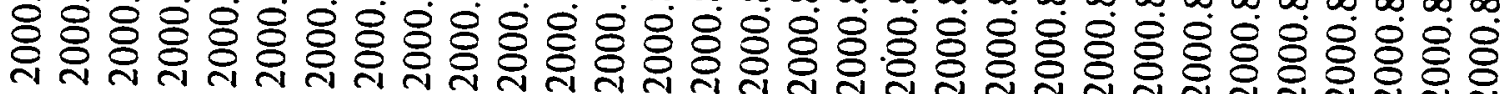

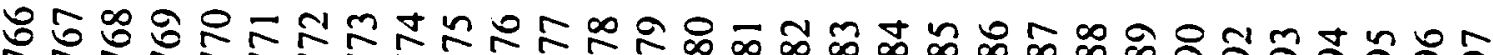

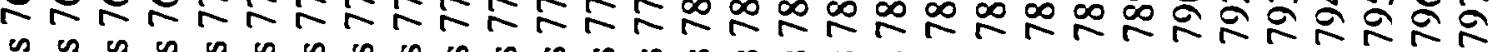

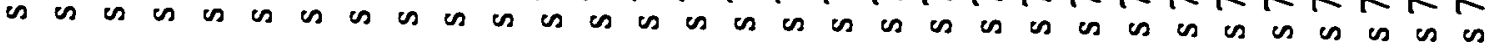




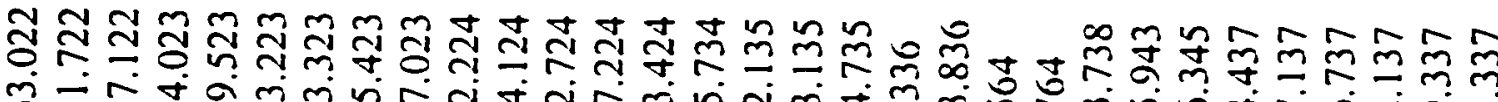

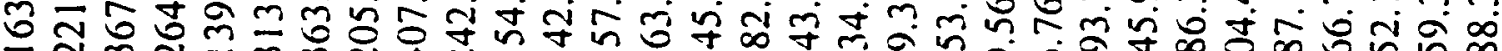
주뀨ำ

0000000000000000000000000000000

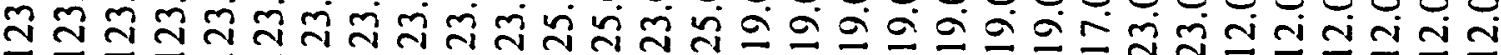

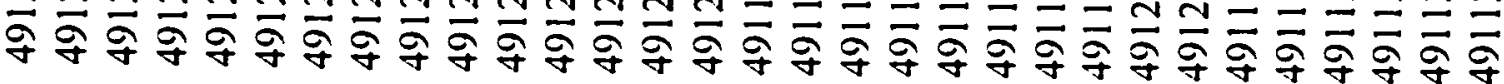

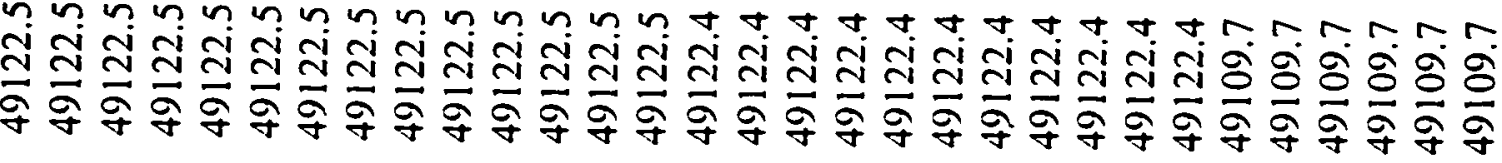

욱엉ㅎㅇ

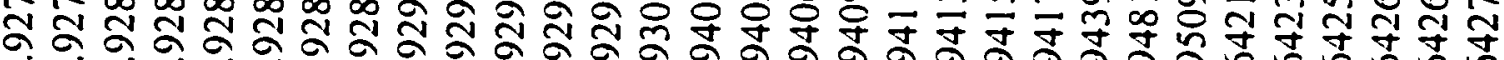

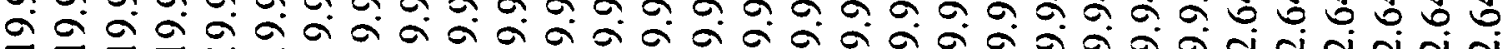
- -

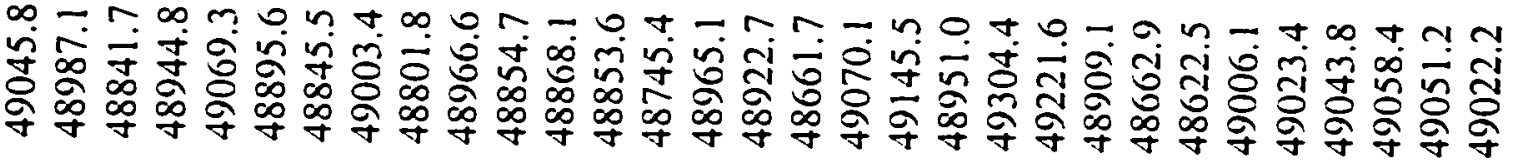

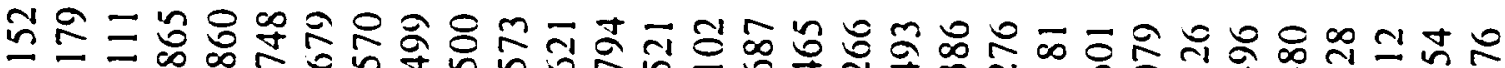
ज.

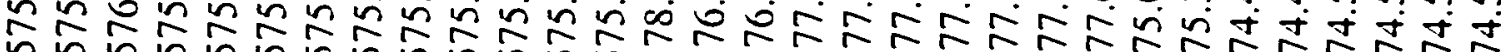

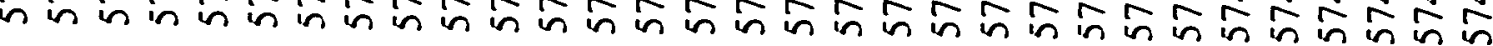

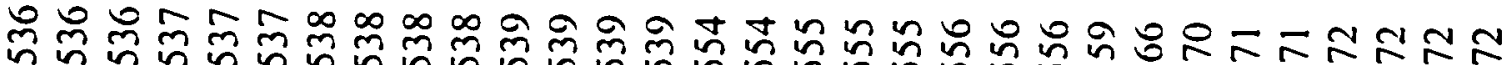

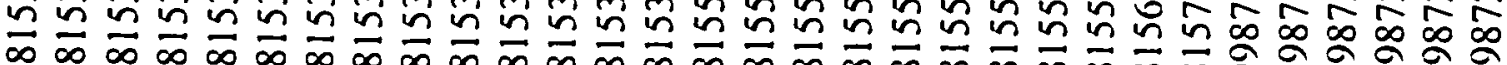

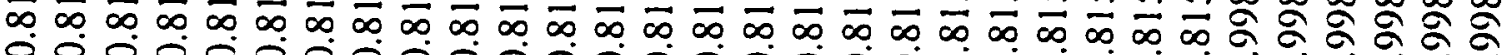
ठ்

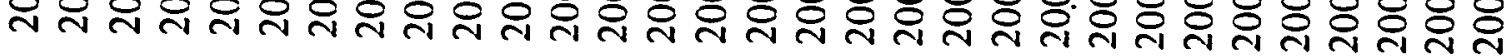

Б

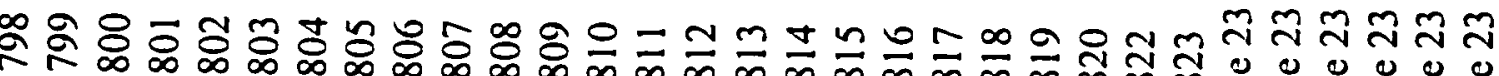

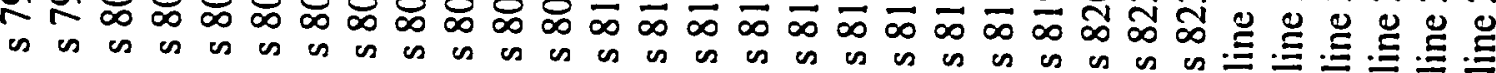




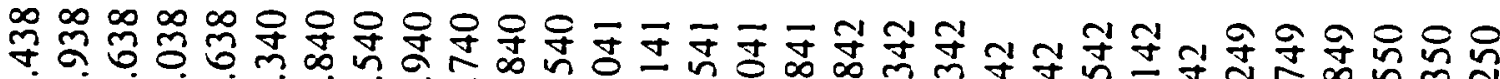

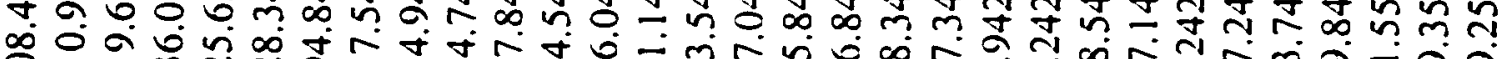

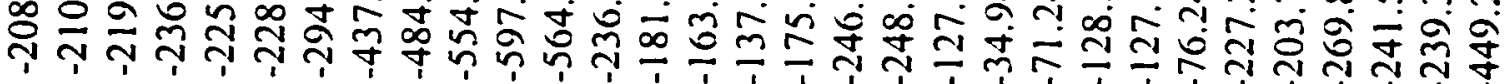

0000000000000000000000000000000 ง

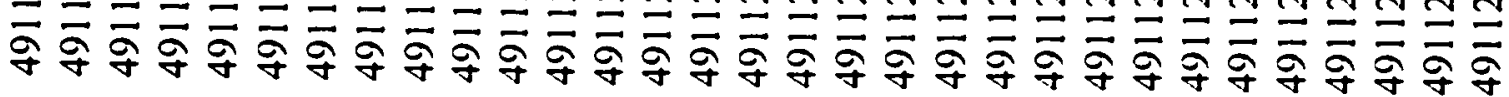

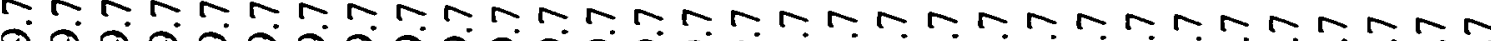

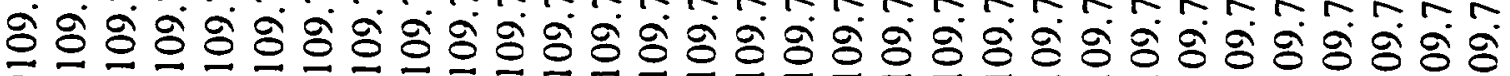

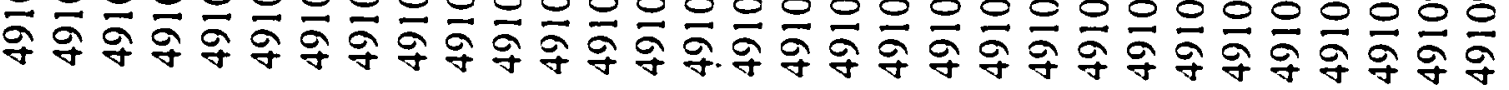

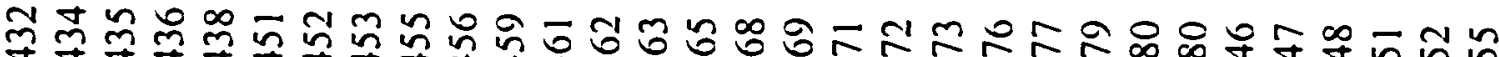

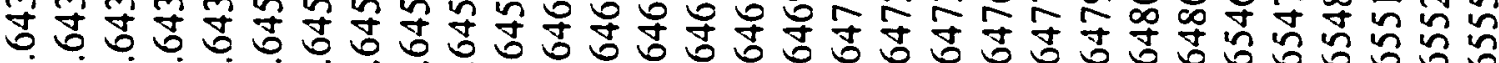
సं

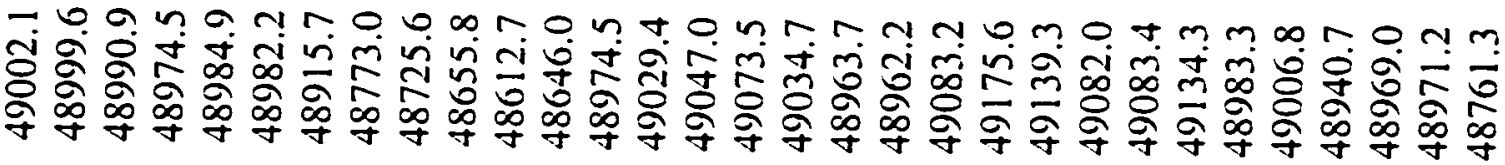

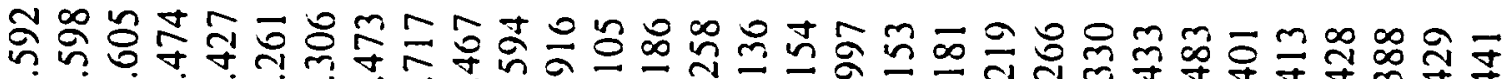

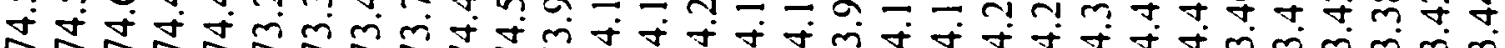

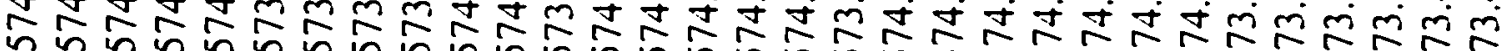

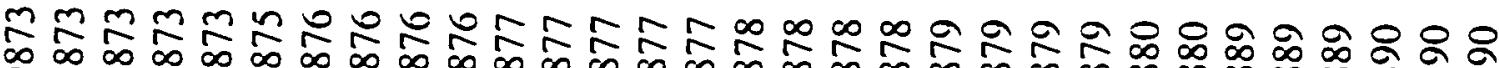

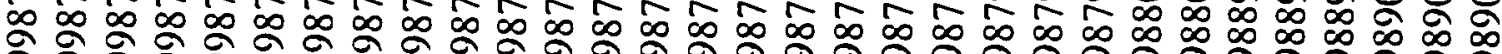
ลे ă ठ்

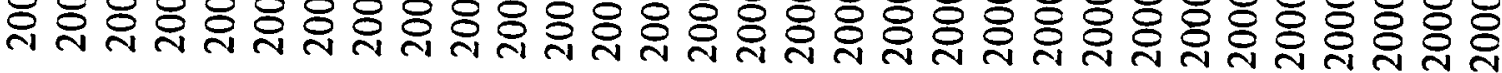

缟守路

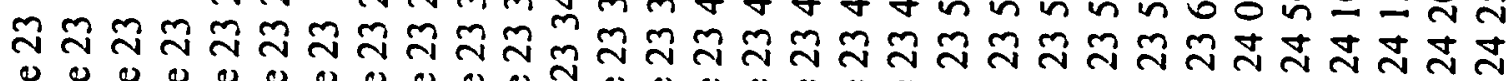

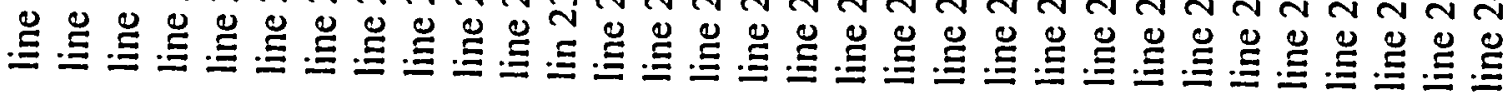




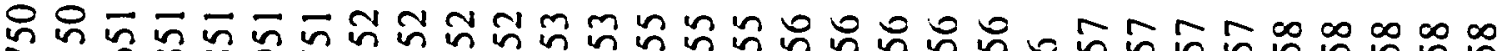
สำ פஸ்

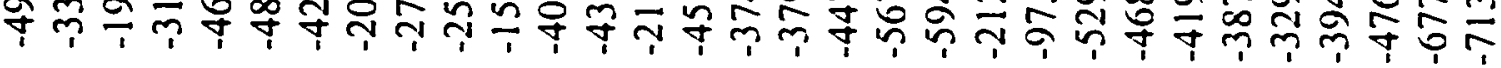

0000000000000000000000000000000

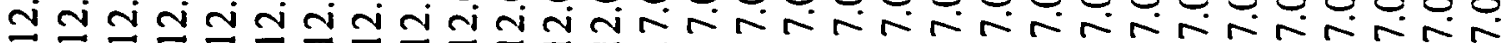

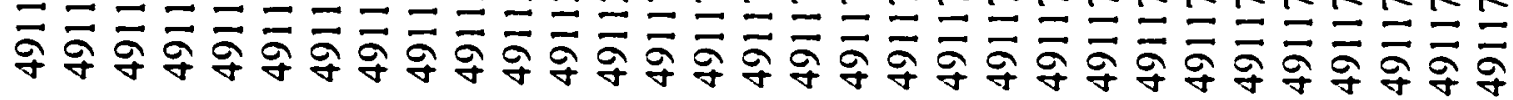

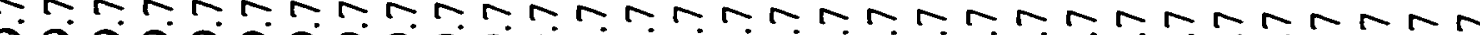

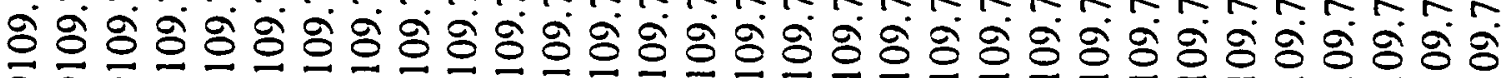

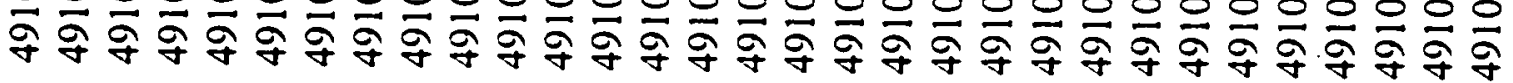

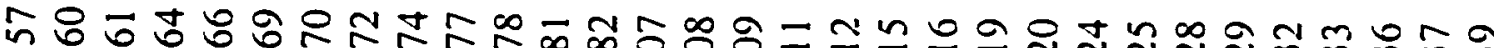
สู้

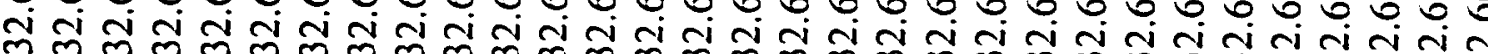

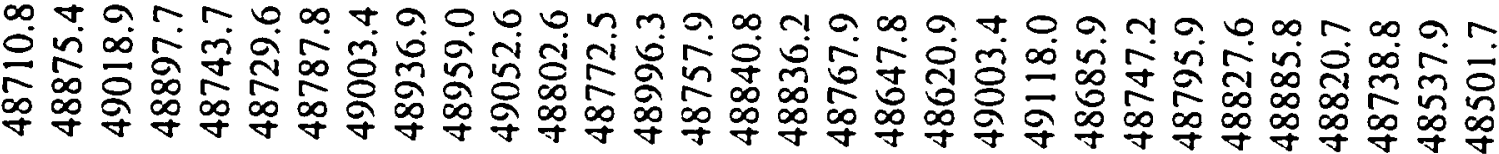

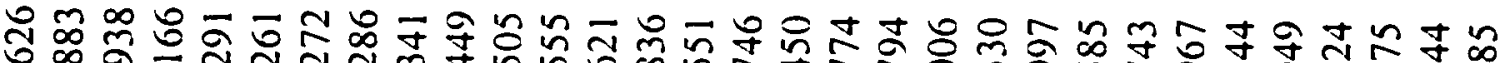
उ.

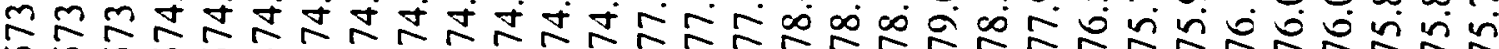

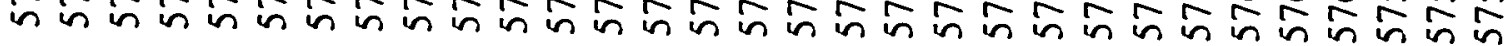

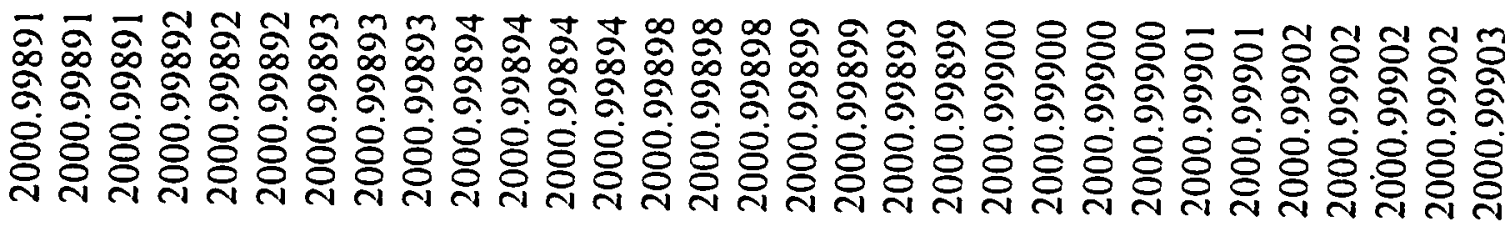

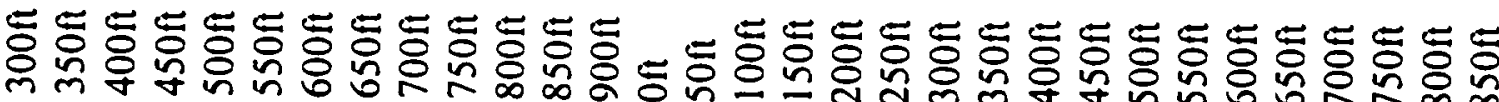
m

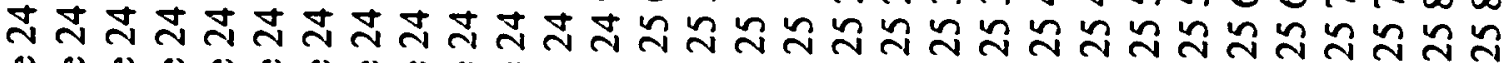

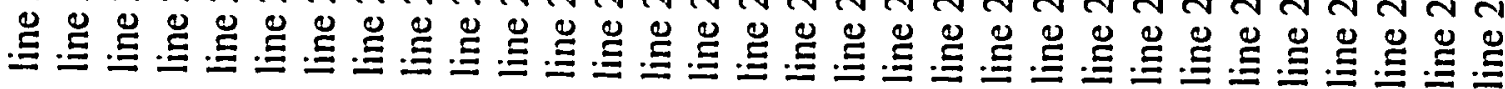




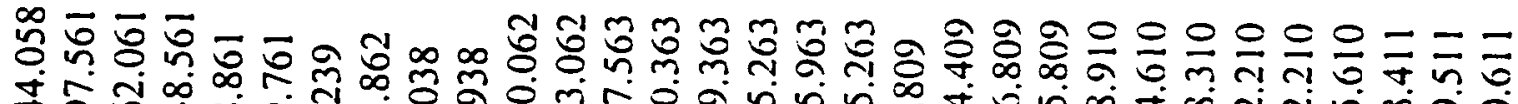

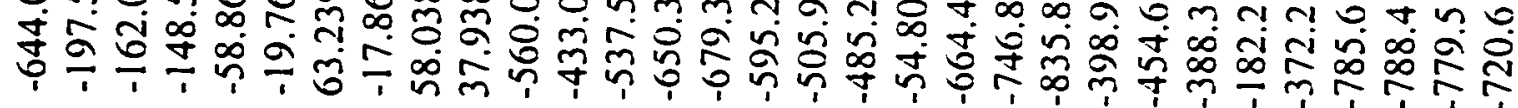

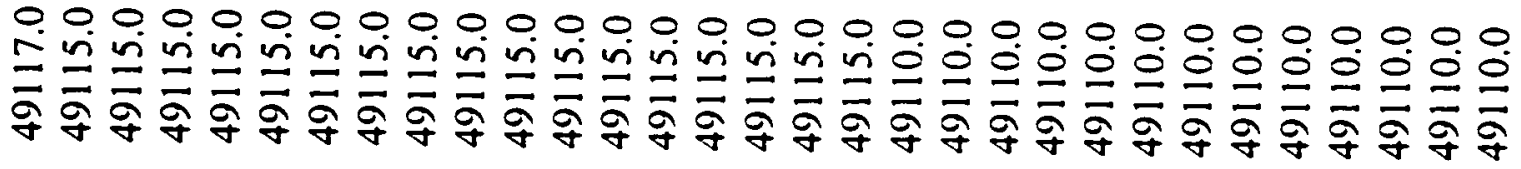

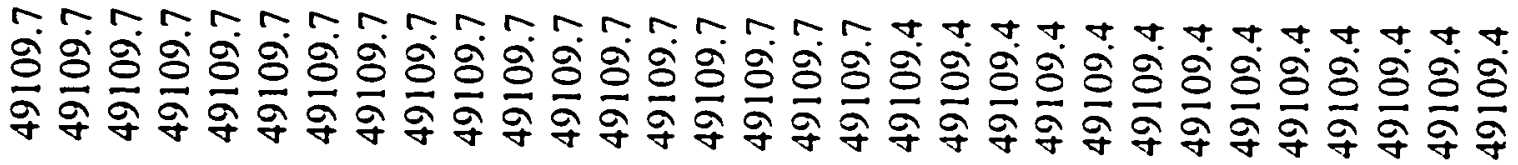

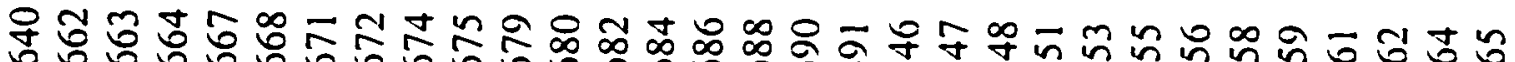

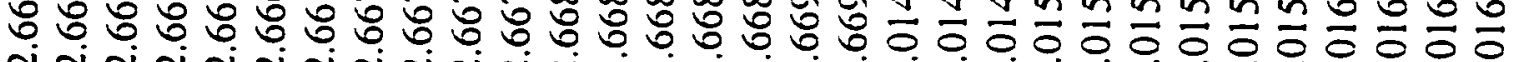
लं

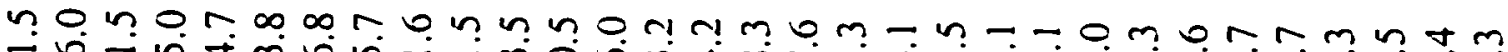

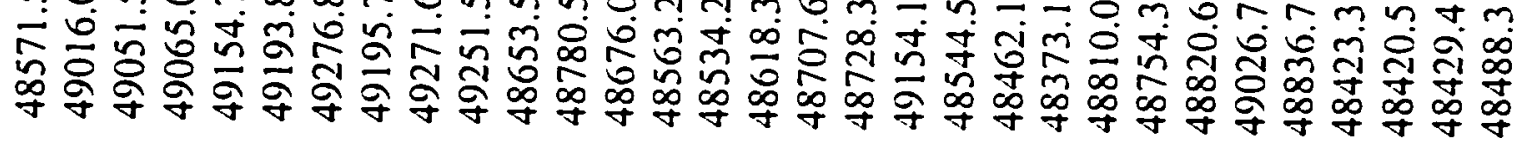

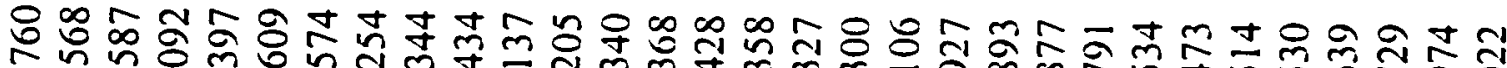
Do

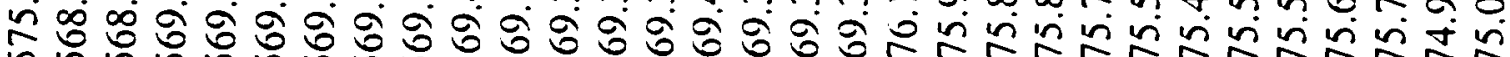

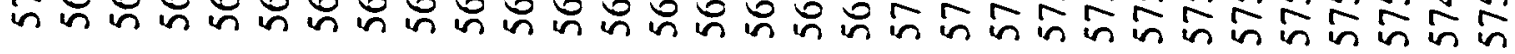

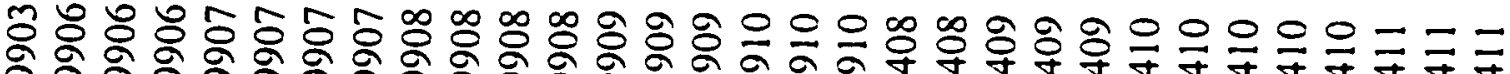
人 O.

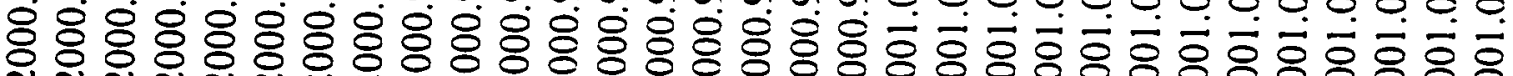

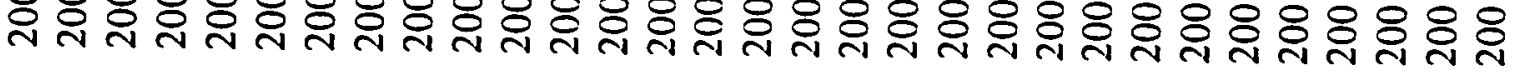

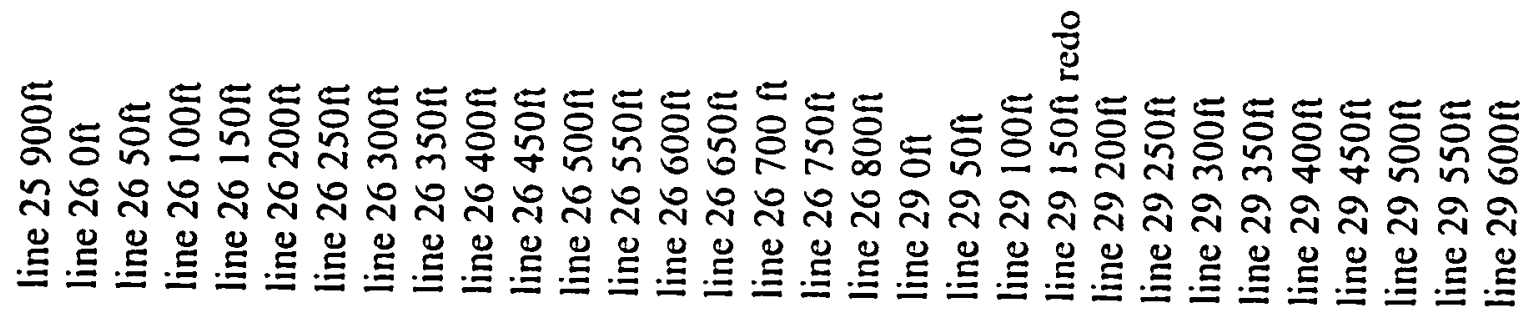




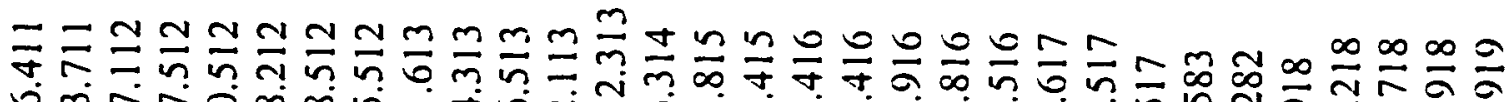

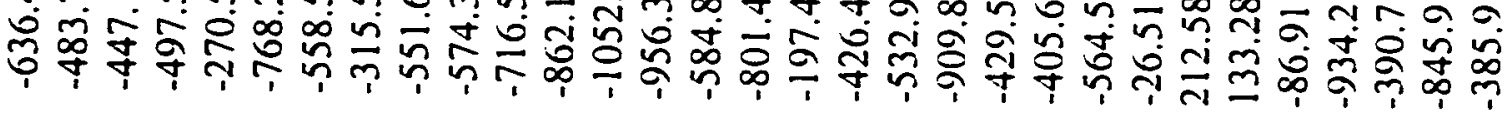

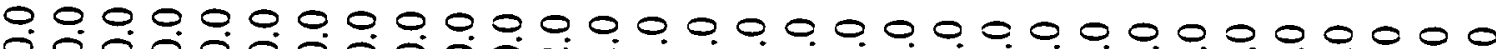

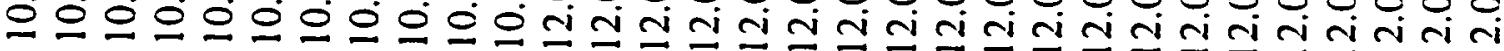

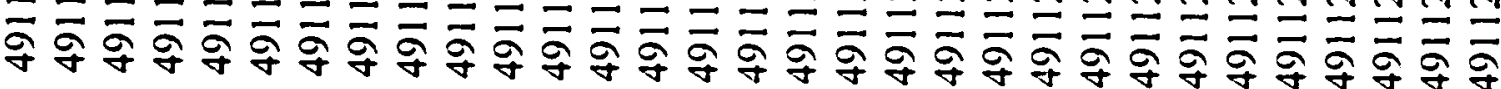

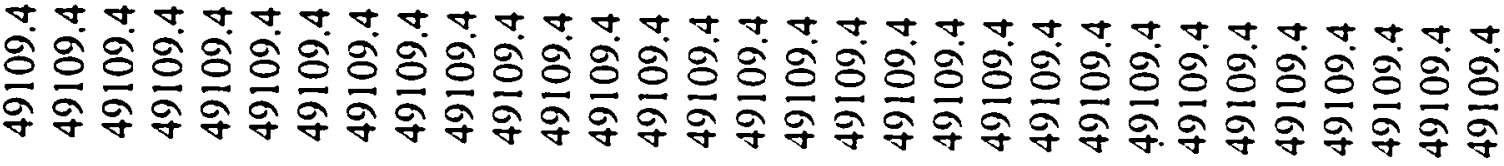

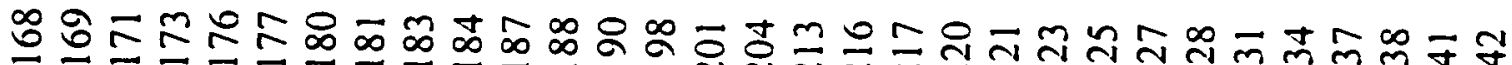

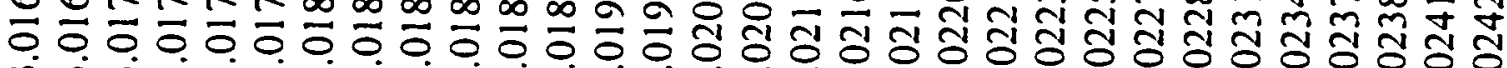
ले

n तथ்

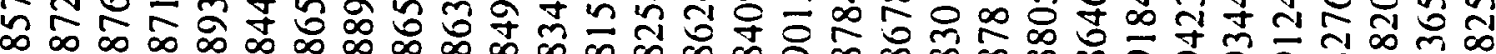

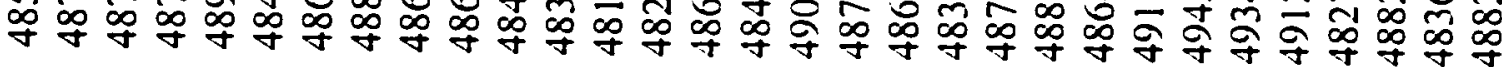

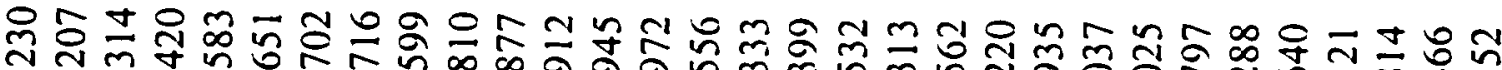
n

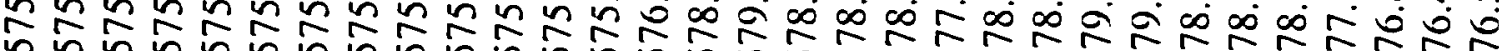

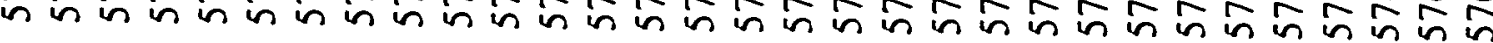

ま

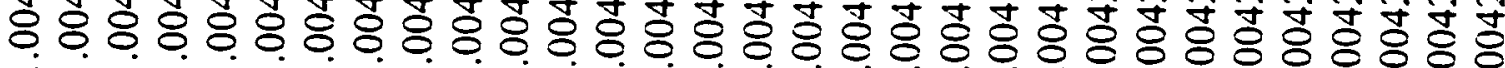

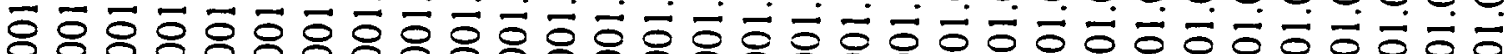

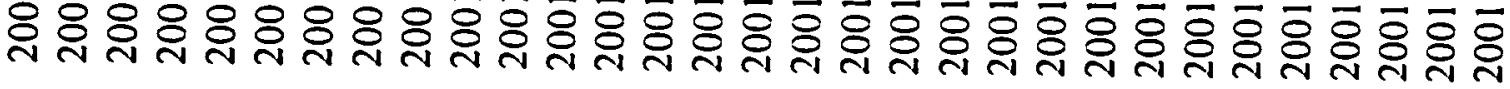

율

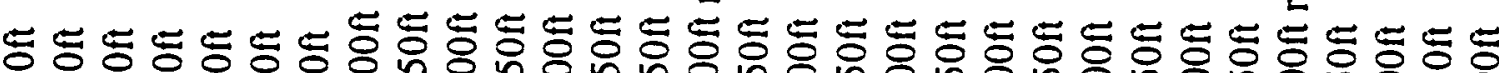

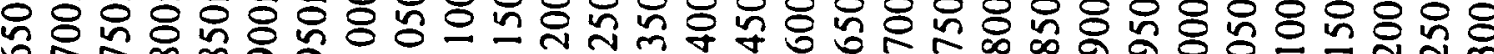

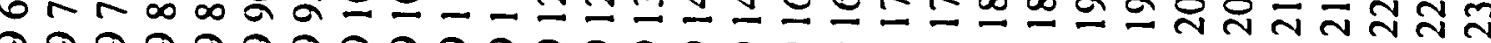

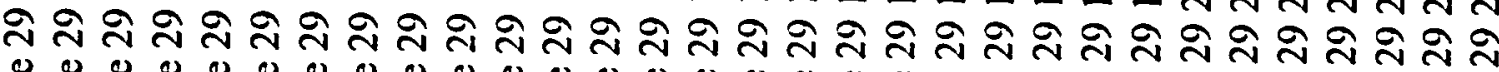

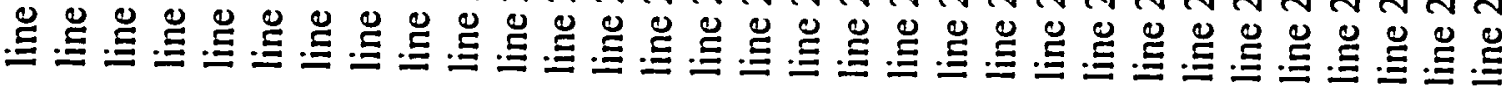


車

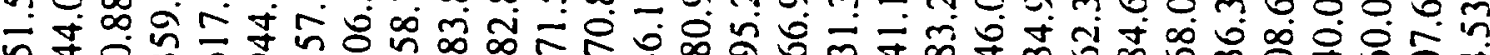
๗ป

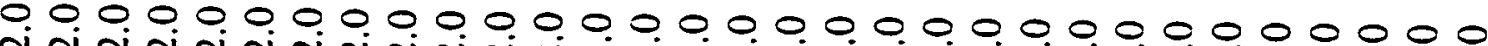

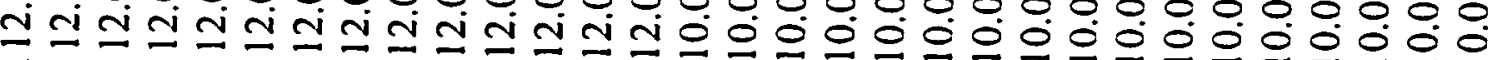

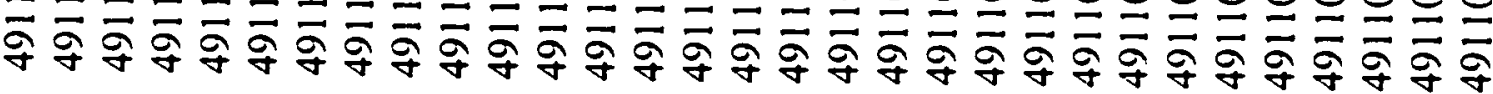

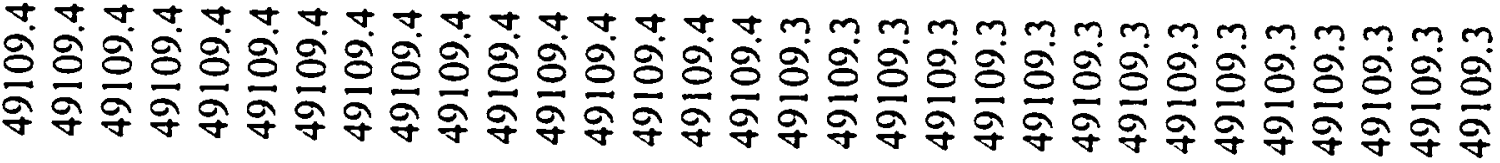

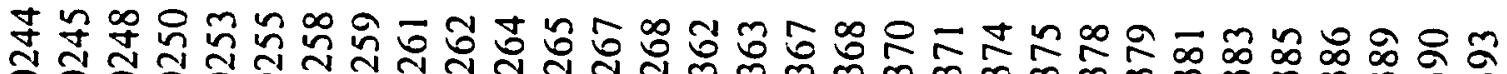

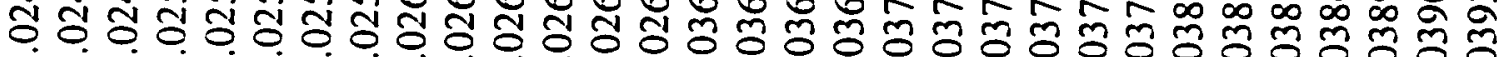

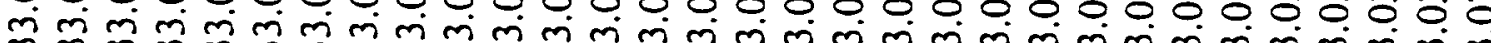

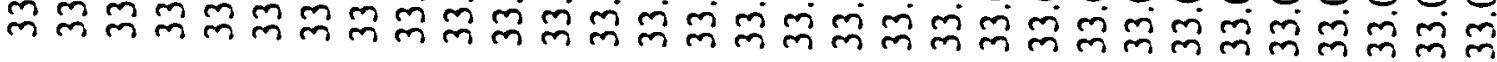

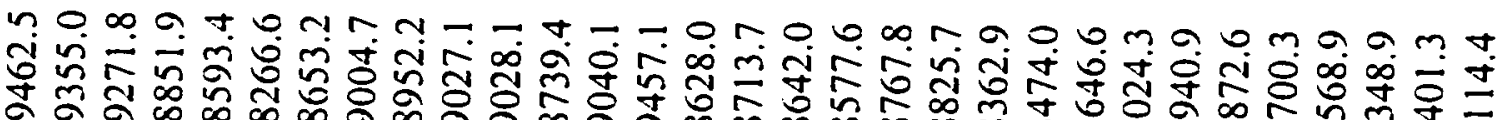

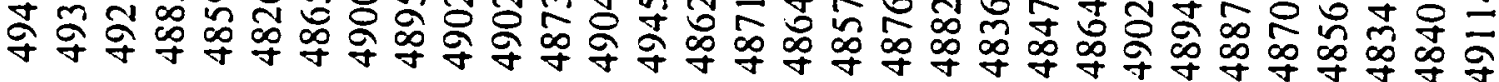

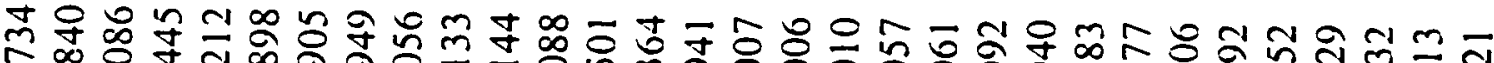

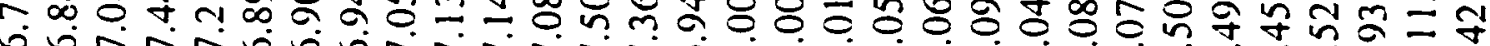
DEN

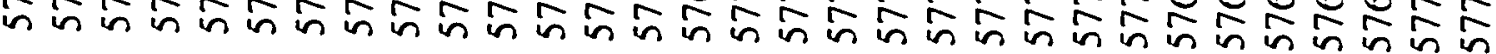

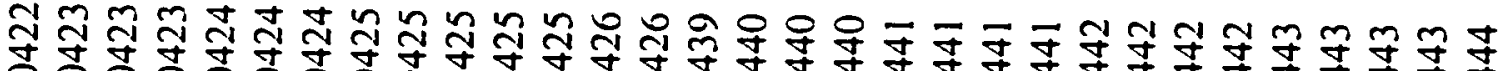

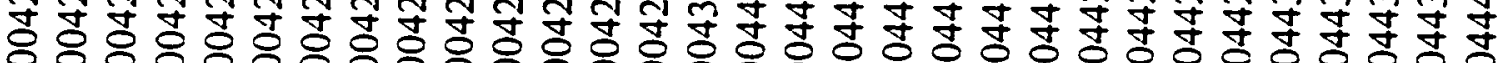

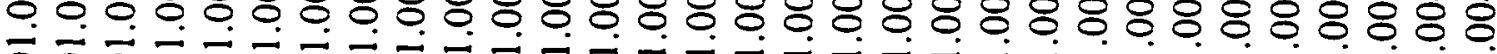

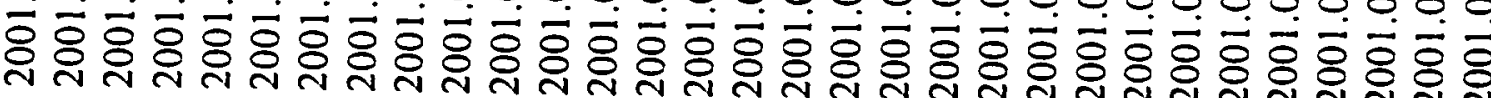

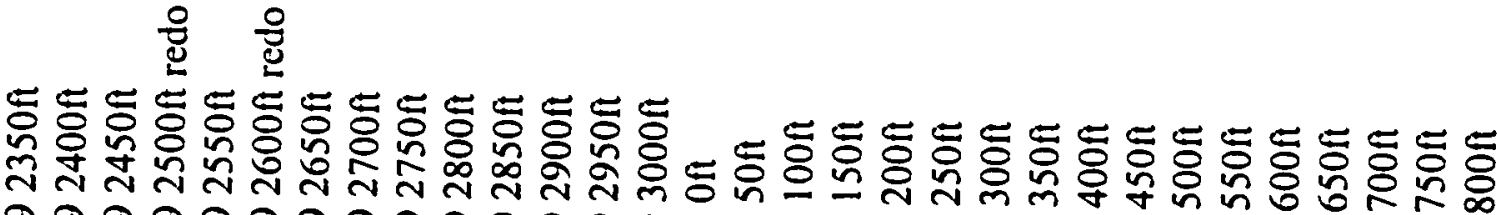

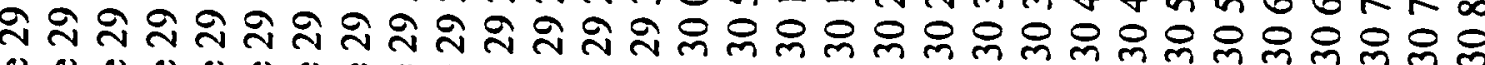

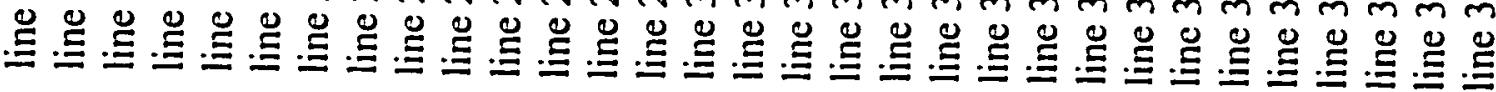




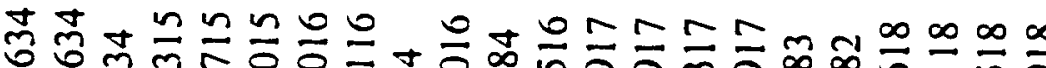

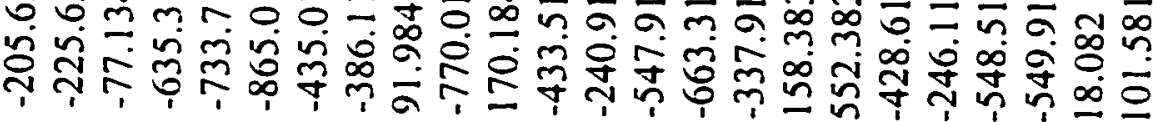

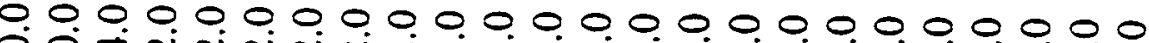

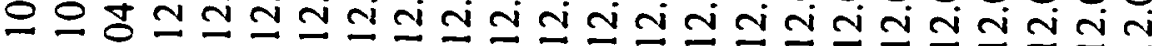

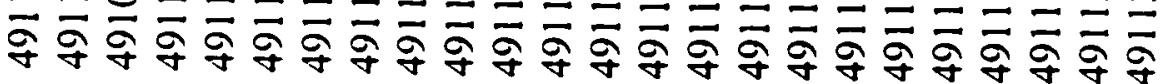

mm m gे

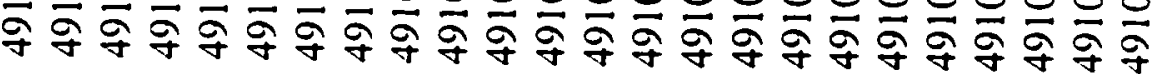

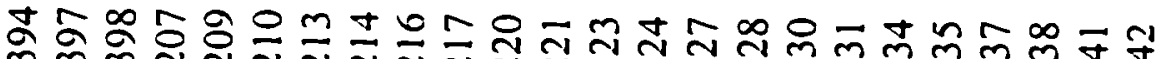
๙ิ mं $\dot{m} \dot{m} \dot{m} \dot{m} \dot{m} \dot{m} \dot{m} \dot{m} \dot{m} \dot{m} \dot{m} \dot{m}$

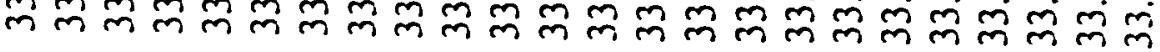

m m

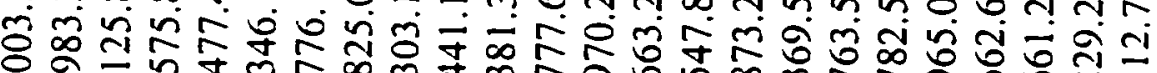

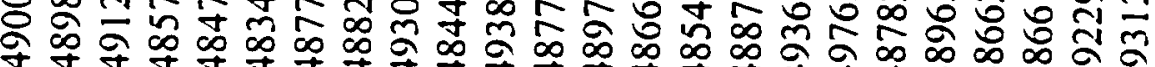

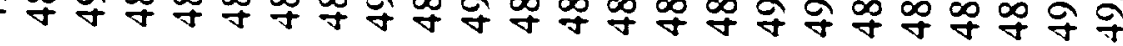

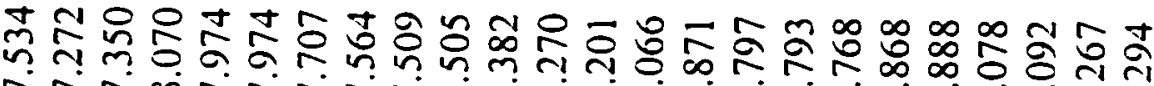

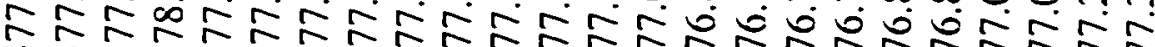

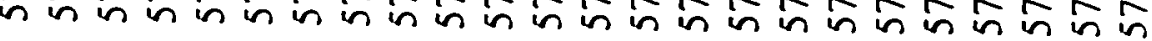

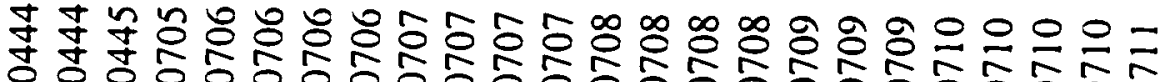

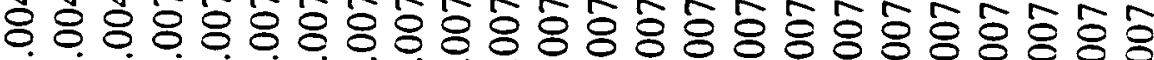

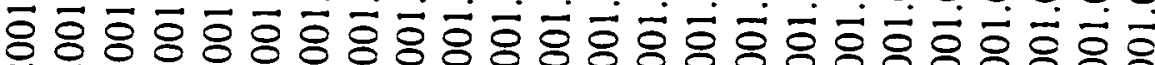

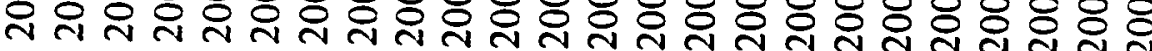

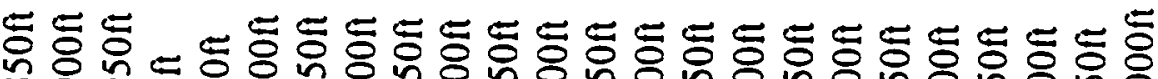

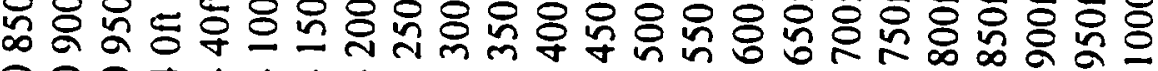
유요

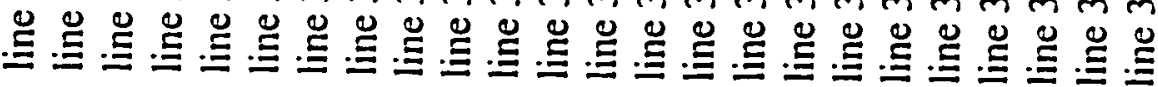


TABLE A2. SEISMIC LINE 1, SHOT 1

\begin{tabular}{lll}
\hline Geophone & Distance from Source $(\mathrm{ft})$ & Arrival Time $(\mathrm{ms})$ \\
\hline 1 & 0 & 0.0 \\
2 & 30 & 27.0 \\
3 & 60 & 29.8 \\
4 & 90 & 38.6 \\
5 & 120 & 41.4 \\
6 & 150 & 46.2 \\
7 & 180 & 53.0 \\
8 & 210 & 58.6 \\
9 & 240 & 65.0 \\
10 & 270 & 68.2 \\
11 & 300 & n.a. \\
12 & 330 & n.a. \\
\hline n.a.=not available. &
\end{tabular}

CALCULATIONS FOR SHOT $(G R A D E=B)$

Slope $=0.1789$

$\mathrm{V}_{1}=1.70 \mathrm{~km} / \mathrm{s}$

$\mathrm{V}_{2}=3.87 \mathrm{~km} / \mathrm{s}$

Minimum depth to basalt bedrock $=44.54 \mathrm{~m}$

TABLE A3. SEISMIC LINE 1, SHOT 2

\begin{tabular}{lll}
\hline Geophone & Distance from Source $(\mathrm{ft})$ & Arrival Time $(\mathrm{ms})$ \\
\hline 1 & 330 & n.a. \\
2 & 300 & n.a. \\
3 & 270 & n.a. \\
4 & 240 & 79.8 \\
5 & 210 & 73.4 \\
6 & 180 & 69.8 \\
7 & 150 & 61.8 \\
8 & 120 & 57.8 \\
9 & 90 & n.a. \\
10 & 60 & n.a. \\
11 & 30 & n.a. \\
12 & 0 & n.a. \\
\hline n.a. $=$ not available. &
\end{tabular}

CALCULATIONS FOR SHOT $($ GRADE $=\mathrm{B})$

Slope $=0.1853$

$\mathrm{V}_{1}=1.65 \mathrm{~km} / \mathrm{s}$

$\mathrm{V}_{2}=3.87 \mathrm{~km} / \mathrm{s}$

Minimum depth to basalt bedrock $=55.35 \mathrm{~m}$ 
TABLE A4. SEISMIC LINE 1, SHOT 3

\begin{tabular}{lll}
\hline Geophone & Distance from Source $(\mathrm{ft})$ & Arrival Time $(\mathrm{ms})$ \\
\hline 1 & 30 & 20.2 \\
2 & 0 & 0.0 \\
3 & 30 & 7.0 \\
4 & 60 & 26.6 \\
5 & 90 & 41.0 \\
6 & 120 & 43.4 \\
7 & 150 & 47.0 \\
8 & 180 & 53.8 \\
9 & 210 & 59.8 \\
10 & 240 & 73.4 \\
11 & 270 & 110.2 \\
12 & 300 & n.a. \\
\hline n.a. $=$ not available. &
\end{tabular}

\section{CALCULATIONS FOR SHOT (GRADE=B)}

Slope $=0.1600$

$\mathrm{V}_{1}=1.91 \mathrm{~km} / \mathrm{s}$

$\mathrm{V}_{2}=3.87 \mathrm{~km} / \mathrm{s}$

Minimum depth to basalt bedrock $=47.35 \mathrm{~m}$

TABLE A5. SEISMIC LINE 1. SHOT 4

\begin{tabular}{lll}
\hline Geophone & Distance from Source $(\mathrm{ft})$ & Arrival Time $(\mathrm{ms})$ \\
\hline 1 & 360 & n.a. \\
2 & 330 & n.a. \\
3 & 300 & 158.6 \\
4 & 270 & 80.2 \\
5 & 240 & 74.6 \\
6 & 210 & 69.4 \\
7 & 180 & 62.2 \\
8 & 150 & 59.4 \\
9 & 120 & 49.4 \\
10 & 90 & 49.4 \\
11 & 60 & 39.8 \\
12 & 30 & 31.8 \\
\hline
\end{tabular}

n.a. $=$ not available.

CALCULATIONS FOR SHOT (GRADE $=\mathrm{B})$

Slope $=0.1800$

$\mathrm{V}_{\mathrm{l}}=1.69 \mathrm{~km} / \mathrm{s}$

$\mathrm{V}_{2}=3.87 \mathrm{~km} / \mathrm{s}$

Minimum depth to basalt bedrock $=50.50 \mathrm{~m}$ 
TABLE A6. SEISMIC LINE 1, SHOT 5

\begin{tabular}{lll}
\hline Geophone & Distance from Source $(\mathrm{ft})$ & Arrival Time $(\mathrm{ms})$ \\
\hline 1 & 0 & 0.0 \\
2 & 30 & 25.8 \\
3 & 60 & 45.4 \\
4 & 90 & 56.2 \\
5 & 120 & 80.2 \\
6 & 150 & 87.0 \\
7 & 180 & 82.6 \\
8 & 210 & n.a. \\
9 & 240 & n.a. \\
10 & 270 & n.a. \\
11 & 300 & n.a. \\
12 & 330 & n.a. \\
\hline
\end{tabular}

n.a. $=$ not available.

\section{CALCULATIONS FOR SHOT $($ GRADE $=\mathrm{C})$}

Slope $=0.5800$

$\mathrm{V}_{\mathrm{I}}=0.53 \mathrm{~km} / \mathrm{s}$

$\mathrm{V}_{2}=3.87 \mathrm{~km} / \mathrm{s}$

Minimum depth to basalt bedrock $=19.94 \mathrm{~m}$

TABLE A7. SEISMIC LINE 1, SHOT 6

\begin{tabular}{lll}
\hline Geophone & Distance from Source $(\mathrm{ft})$ & Arrival Time $(\mathrm{ms})$ \\
\hline 1 & 330 & 113.0 \\
2 & 300 & 85.4 \\
3 & 270 & 83.4 \\
4 & 240 & 76.6 \\
5 & 210 & 71.4 \\
6 & 180 & 67.4 \\
7 & 150 & 65.8 \\
8 & 120 & 2.2 \\
9 & 90 & 1.8 \\
10 & 60 & 43.8 \\
11 & 30 & 1.0 \\
12 & 0 & 0.0 \\
\hline n.a. =not available. &
\end{tabular}

\section{CALCULATIONS FOR SHOT (GRADE $=C$ )}

Slope $=0.1440$

$\mathrm{V}_{1}=2.12 \mathrm{~km} / \mathrm{s}$

$\mathrm{V}_{2}=3.87 \mathrm{~km} / \mathrm{s}$

Minimum depth to basalt bedrock $=78.11 \mathrm{~m}$ 
TABLE A8. SEISMIC LINE 1, SHOT 7

\begin{tabular}{lll}
\hline Geophone & Distance from Source $(\mathrm{ft})$ & Arrival Time $(\mathrm{ms})$ \\
\hline 1 & 30 & 24.6 \\
2 & 0 & 0.0 \\
3 & 30 & 5.0 \\
4 & 60 & 23.0 \\
5 & 90 & 47.8 \\
6 & 120 & 50.6 \\
7 & 150 & 63.0 \\
8 & 180 & n.a. \\
9 & 210 & n.a. \\
10 & 240 & n.a. \\
11 & 270 & n.a. \\
12 & 300 & n.a. \\
\hline n.a.=not available. &
\end{tabular}

\section{CALCULATIONS FOR SHOT (GRADE $=\mathrm{C}$ )}

Slope $=0.4539$

$\mathrm{V}_{\mathrm{l}}=0.67 \mathrm{~km} / \mathrm{s}$

$V_{2}=3.87 \mathrm{~km} / \mathrm{s}$

Minimum depth to basalt bedrock $=17.45 \mathrm{~m}$

TABLE A9. SEISMIC LINE 1, SHOT 8

\begin{tabular}{lll}
\hline Geophone & Distance from Source $(\mathrm{ft})$ & Arrival Time $(\mathrm{ms})$ \\
\hline 1 & 360 & n.a. \\
2 & 330 & n.a. \\
3 & 300 & n.a. \\
4 & 270 & n.a. \\
5 & 240 & 90.6 \\
6 & 210 & 87.8 \\
7 & 180 & 76.6 \\
8 & 150 & n.a. \\
9 & 120 & n.a. \\
10 & 90 & 55.4 \\
11 & 60 & n.a. \\
12 & 30 & 25.0 \\
\hline
\end{tabular}

n.a. $=$ not available.

\section{CALCULATIONS FOR SHOT (GRADE $=\mathrm{B})$}

Slope $=0.2448$

$\mathrm{V}_{1}=1.25 \mathrm{~km} / \mathrm{s}$

$\mathrm{V}_{2}=3.87 \mathrm{~km} / \mathrm{s}$

Minimum depth to basalt bedrock $=47.15 \mathrm{~m}$ 
TABLE A10. SEISMIC LINE 2, SHOT 1

\begin{tabular}{lll}
\hline Geophone & Distance from Source (ft) & Arrival Time $(\mathrm{ms})$ \\
\hline 1 & 0 & n.a. \\
2 & 30 & 1.4 \\
3 & 60 & 24.6 \\
4 & 90 & 26.2 \\
5 & 120 & 28.6 \\
6 & 150 & 31.0 \\
7 & 180 & 54.6 \\
8 & 210 & n.a. \\
9 & 240 & n.a. \\
10 & 270 & n.a. \\
11 & 300 & n.a. \\
12 & 330 & n.a. \\
\hline
\end{tabular}

n.a.=not available.

CALCULATIONS FOR SHOT (GRADE=A)

Slopes $=0.7733,0.0720$

$\mathrm{V}_{1}=0.39 \mathrm{~km} / \mathrm{s}$

$\mathrm{V}_{2}=4.23 \mathrm{~km} / \mathrm{s}$

Depth to basalt bedrock $=3.97 \mathrm{~m}$

TABLE A11. SEISMIC LINE 2, SHOT 2

\begin{tabular}{lll}
\hline Geophone & Distance from Source (ft) & Arrival Time $(\mathrm{ms})$ \\
\hline 1 & 330 & n.a. \\
2 & 300 & n.a. \\
3 & 270 & 108.2 \\
4 & 240 & 115.0 \\
5 & 210 & 88.6 \\
6 & 180 & 90.2 \\
7 & 150 & 49.0 \\
8 & 120 & n.a. \\
9 & 90 & n.a. \\
10 & 60 & 40.6 \\
11 & 30 & 2.6 \\
12 & 0 & 0.0 \\
\hline n.a. $=$ not available. &
\end{tabular}

CALCULATIONS FOR SHOT $($ GRADE $=C)$

Slope $=0.4115$

$\mathrm{V}_{1}=0.74 \mathrm{~km} / \mathrm{s}$

$\mathrm{V}_{2}=3.87 \mathrm{~km} / \mathrm{s}$

Minimum depth to basalt bedrock $=32.81 \mathrm{~m}$ 
TABLE A 12. SEISMIC LINE 2, SHOT 3

\begin{tabular}{lll}
\hline Geophone & Distance from Source $(\mathrm{ft})$ & Arrival Time $(\mathrm{ms})$ \\
\hline 1 & 30 & 23.4 \\
2 & 0 & 0.0 \\
3 & 30 & 13.0 \\
4 & 60 & 19.8 \\
5 & 90 & 33.0 \\
6 & 120 & 42.2 \\
7 & 150 & 81.4 \\
8 & 180 & n.a. \\
9 & 210 & n.a. \\
10 & 240 & 109.8 \\
11 & 270 & n.a. \\
12 & 300 & n.a. \\
\hline n.a.=not available. &
\end{tabular}

\section{CALCULATIONS FOR SHOT (GRADE=A)}

Slope $=0.3480$

$\mathrm{V}_{1}=0.88 \mathrm{~km} / \mathrm{s}$

$\mathrm{V}_{2}=3.87 \mathrm{~km} / \mathrm{s}$

Minimum depth to basalt bedrock $=14.73 \mathrm{~m}$

TABLE A13. SEISMIC LINE 2, SHOT 4

\begin{tabular}{lll}
\hline Geophone & Distance from Source $(\mathrm{ft})$ & Arrival Time $(\mathrm{ms})$ \\
\hline 1 & 360 & n.a. \\
2 & 330 & n.a. \\
3 & 300 & 99.8 \\
4 & 270 & 140.6 \\
5 & 240 & 103.8 \\
6 & 210 & 108.6 \\
7 & 180 & 97.4 \\
8 & 150 & n.a. \\
9 & 120 & n.a. \\
10 & 90 & 63.8 \\
11 & 60 & 31.8 \\
12 & 30 & 23.4 \\
\hline n.a.=not available. &
\end{tabular}

CALCULATIONS FOR SHOT $(\mathrm{GRADE}=\mathrm{A})$

Slope $=0.5029$

$\mathrm{V}_{1}=0.61 \mathrm{~km} / \mathrm{s}$

$\mathrm{V}_{2}=3.87 \mathrm{~km} / \mathrm{s}$

Minimum depth to basalt bedrock $=36.62 \mathrm{~m}$ 
TABLE A14. SEISMIC LINE 6, SHOT 1

\begin{tabular}{lll}
\hline Geophone & Distance from Source $(\mathrm{ft})$ & Arrival Time $(\mathrm{ms})$ \\
\hline 1 & 0 & 0.0 \\
2 & 10 & 7.8 \\
3 & 20 & 16.6 \\
4 & 30 & 27.8 \\
5 & 40 & 40.6 \\
6 & 50 & 55.0 \\
7 & 60 & 57.0 \\
8 & 70 & n.a. \\
9 & 80 & n.a. \\
10 & 90 & 79.0 \\
11 & 100 & 86.6 \\
12 & 110 & n.a. \\
\hline
\end{tabular}

n.a. $=$ not available.

CALCULATIONS FOR SHOT $($ GRADE $=$ A)

Slope $=0.8858$

$\mathrm{V}_{1}=0.34 \mathrm{~km} / \mathrm{s}$

$\mathrm{V}_{2}=3.87 \mathrm{~km} / \mathrm{s}$

Minimum depth to basalt bedrock $=13.60 \mathrm{~m}$

TABLE A15. SEISMIC LINE 6, SHOT 2

\begin{tabular}{lll}
\hline Geophone & Distance from Source $(\mathrm{ft})$ & Arrival Time $(\mathrm{ms})$ \\
\hline 1 & 110 & 80.2 \\
2 & 100 & 69.4 \\
3 & 90 & 66.6 \\
4 & 80 & 62.6 \\
5 & 70 & 57.0 \\
6 & 60 & 43.8 \\
7 & 50 & 37.4 \\
8 & 40 & n.a. \\
9 & 30 & n.a. \\
10 & 20 & 15.4 \\
11 & 10 & 5.0 \\
12 & 0 & 0.0 \\
\hline n.a. $=$ not available. &
\end{tabular}

\section{CALCULATIONS FOR SHOT $($ GRADE $=\mathrm{A})$}

Slope $=0.7377$

$\mathrm{V}_{\mathrm{l}}=0.41 \mathrm{~km} / \mathrm{s}$

$\mathrm{V}_{2}=3.87 \mathrm{~km} / \mathrm{s}$

Minimum depth to basalt bedrock $=14.87 \mathrm{~m}$ 
TABLE A 16. SEISMIC LINE 7, SHOT 1

\begin{tabular}{lll}
\hline Geophone & Distance from Source $(\mathrm{ft})$ & Arrival Time $(\mathrm{ms})$ \\
\hline 1 & 0 & 0.0 \\
2 & 30 & 25.4 \\
3 & 60 & 45.8 \\
4 & 90 & 72.6 \\
5 & 120 & 81.8 \\
6 & 150 & 119.4 \\
7 & 180 & 137.0 \\
8 & 210 & n.a. \\
9 & 240 & n.a. \\
10 & 270 & n.a. \\
11 & 300 & n.a. \\
12 & 330 & n.a. \\
\hline n.a. $=$ not available. &
\end{tabular}

CALCULATIONS FOR SHOT (GRADE $=\mathrm{A})$

Slope $=0.7701$

$\mathrm{V}_{1}=0.40 \mathrm{~km} / \mathrm{s}$

$V_{2}=3.87 \mathrm{~km} / \mathrm{s}$

Minimum depth to basalt bedrock $=24.44 \mathrm{~m}$

TABLE A17. SEISMIC LINE 7, SHOT 2

\begin{tabular}{lll}
\hline Geophone & Distance from Source $(\mathrm{ft})$ & Arrival Time $(\mathrm{ms})$ \\
\hline 1 & 240 & 116.2 \\
2 & 210 & 111.8 \\
3 & 180 & 100.6 \\
4 & 150 & 96.2 \\
5 & 120 & 94.2 \\
6 & 90 & 88.2 \\
7 & 60 & 80.2 \\
8 & 30 & n.a. \\
9 & 0 & 0.0 \\
10 & 30 & n.a. \\
11 & 60 & n.a. \\
12 & 90 & n.a. \\
\hline n.a. $=$ not available. &
\end{tabular}

CALCULATIONS FOR SHOT $(G R A D E=B)$

Slope $=0.1924$

$\mathrm{V}_{1}=1.58 \mathrm{~km} / \mathrm{s}$

$\mathrm{V}_{2}=3.87 \mathrm{~km} / \mathrm{s}$

Minimum depth to basalt bedrock $=84.49 \mathrm{~m}$ 
TABLE A18. SEISMIC LINE 7, SHOT 3

\begin{tabular}{lll}
\hline Geophone & Distance from Source $(\mathrm{ft})$ & Arrival Time $(\mathrm{ms})$ \\
\hline 1 & 0 & 0.0 \\
2 & 30 & 39.0 \\
3 & 60 & 51.8 \\
4 & 90 & 63.4 \\
5 & 120 & 68.2 \\
6 & 150 & 71.0 \\
7 & 180 & 74.6 \\
8 & 210 & n.a. \\
9 & 240 & n.a. \\
10 & 270 & n.a. \\
11 & 300 & n.a. \\
12 & 330 & n.a. \\
\hline
\end{tabular}

n.a. $=$ not available.

CALCULATIONS FOR SHOT $($ GRADE $=C)$

Slope $=0.1213$

$V_{1}=2.51 \mathrm{~km} / \mathrm{s}$

$\mathrm{V}_{2}=3.87 \mathrm{~km} / \mathrm{s}$

Minimum depth to basalt bedrock $=99.86 \mathrm{~m}$

TABLE A19. SEISMIC LINE 7, SHOT 4

\begin{tabular}{lll}
\hline Geophone & Distance from Source $(\mathrm{ft})$ & Arrival Time $(\mathrm{ms})$ \\
\hline 1 & 240 & 86.6 \\
2 & 210 & 83.0 \\
3 & 180 & 68.6 \\
4 & 150 & 65.4 \\
5 & 120 & 63.8 \\
6 & 90 & 57.8 \\
7 & 60 & 50.2 \\
8 & 30 & 31.0 \\
9 & 0 & 0.0 \\
10 & 30 & 30.2 \\
11 & 60 & n.a. \\
12 & 90 & n.a. \\
\hline
\end{tabular}

n.a. $=$ not available.

CALCULATIONS FOR SHOT $($ GRADE $=\mathrm{B})$

Slope $=0.2700$

$\mathrm{V}_{1}=1.13 \mathrm{~km} / \mathrm{s}$

$\mathrm{V}_{2}=3.87 \mathrm{~km} / \mathrm{s}$

Minimum depth to basalt bedrock $=39.96 \mathrm{~m}$ 
TABLE A20. SEISMIC LINE 7, SHOT 5

\begin{tabular}{lll}
\hline Geophone & Distance from Source (ft) & Arrival Time (ms) \\
\hline 1 & 330 & n.a. \\
2 & 300 & 167.4 \\
3 & 270 & 162.2 \\
4 & 240 & 159.8 \\
5 & 210 & 149.8 \\
6 & 180 & 139.4 \\
7 & 150 & 103.8 \\
8 & 120 & n.a. \\
9 & 90 & n.a. \\
10 & 60 & 5.1 \\
11 & 30 & n.a. \\
12 & 0 & 0.0 \\
\hline
\end{tabular}

n.a. $=$ not available.

CALCULATIONS FOR SHOT (GRADE=B)

Slope $=0.2280$

$\mathrm{V}_{1}=1.34 \mathrm{~km} / \mathrm{s}$

$\mathrm{V}_{2}=3.87 \mathrm{~km} / \mathrm{s}$

Minimum depth to basalt bedrock $=102.43 \mathrm{~m}$

TABLE A21. SEISMIC LINE 7, SHOT 6

\begin{tabular}{lll}
\hline Geophone & Distance from Source $(\mathrm{ft})$ & Arrival Time $(\mathrm{ms})$ \\
\hline 1 & 240 & 99.8 \\
2 & 210 & 98.2 \\
3 & 180 & 69.4 \\
4 & 150 & 58.2 \\
5 & 120 & 52.6 \\
6 & 90 & 46.6 \\
7 & 60 & 23.8 \\
8 & 30 & n.a. \\
9 & 0 & 0.0 \\
10 & 30 & 52.6 \\
11 & 60 & n.a. \\
12 & 90 & n.a. \\
\hline n.a.=not available &
\end{tabular}

CALCULATIONS FOR SHOT

Slope $=0.1933$

$\mathrm{V}_{1}=1.58 \mathrm{~km} / \mathrm{s}$

$\mathrm{V}_{2}=3.87 \mathrm{~km} / \mathrm{s}$

Minimum depth to basalt bedrock $=40.06 \mathrm{~m}$ 
TABLE A22. SEISMIC LINE 7, SHOT 7

\begin{tabular}{lll}
\hline Geophone & Distance from Source $(\mathrm{ft})$ & Arrival Time $(\mathrm{ms})$ \\
\hline 1 & 330 & 158.6 \\
2 & 300 & 155.4 \\
3 & 270 & 153.0 \\
4 & 240 & 147.0 \\
5 & 210 & 129.0 \\
6 & 180 & 73.4 \\
7 & 150 & 32.2 \\
8 & 120 & 1.4 \\
9 & 90 & n.a. \\
10 & 60 & 54.2 \\
11 & 30 & n.a. \\
12 & 0 & 0.0 \\
\hline n.a. =not available. &
\end{tabular}

\section{CALCULATIONS FOR SHOT (GRADE=B)}

Slope $=0.0933$

$\mathrm{V}_{1}=0.52 \mathrm{~km} / \mathrm{s}$

$\mathrm{V}_{2}=3.27 \mathrm{~km} / \mathrm{s}$

Depth to basalt bedrock $=33.76 \mathrm{~m}$

TABLE A23. SEISMIC LINE 7, SHOT 8

\begin{tabular}{lll}
\hline Geophone & Distance from Source $(\mathrm{ft})$ & Arrival Time $(\mathrm{ms})$ \\
\hline 1 & 0 & 0.0 \\
2 & 30 & 24.2 \\
3 & 60 & 50.6 \\
4 & 90 & 73.4 \\
5 & 120 & 93.4 \\
6 & 150 & 102.6 \\
7 & 180 & 113.0 \\
8 & 210 & n.a. \\
9 & 240 & n.a. \\
10 & 270 & n.a. \\
11 & 300 & n.a. \\
12 & 330 & n.a. \\
\hline
\end{tabular}

n.a. =not available.

CALCULATIONS FOR SHOT $($ GRADE $=B$ )

Slope $=0.3267$

$\mathrm{V}_{1}=0.93 \mathrm{~km} / \mathrm{s}$

$\mathrm{V}_{2}=3.87 \mathrm{~km} / \mathrm{s}$

Minimum depth to basalt bedrock $=47.51 \mathrm{~m}$ 
TABLE A24. SEISMIC LINE 7, SHOT 9

\begin{tabular}{lll}
\hline Geophone & Distance from Source (ft) & Arrival Time (ms) \\
\hline 1 & 0 & 0.0 \\
2 & 30 & 30.2 \\
3 & 60 & 40.6 \\
4 & 90 & 52.6 \\
5 & 120 & 52.2 \\
6 & 150 & 49.8 \\
7 & 180 & 62.2 \\
8 & 210 & n.a. \\
9 & 240 & n.a. \\
10 & 270 & n.a. \\
11 & 300 & n.a. \\
12 & 330 & n.a. \\
\hline n.a.=not available. &
\end{tabular}

CALCULATIONS FOR SHOT (GRADE $=D$ )

Slope $=0.1800$

$\mathrm{V}_{\mathrm{I}}=1.69 \mathrm{~km} / \mathrm{s}$

$\mathrm{V}_{2}=3.87 \mathrm{~km} / \mathrm{s}$

Minimum depth to basalt bedrock $=45.23 \mathrm{~m}$

TABLE A25. SEISMIC LINE 8, SHOT 1

\begin{tabular}{lll}
\hline Geophone & Distance from Source (ft) & Arrival Time $(\mathrm{ms})$ \\
\hline 1 & 0 & 0.0 \\
2 & 10 & 7.4 \\
3 & 20 & 17.0 \\
4 & 30 & 25.8 \\
5 & 40 & 36.2 \\
6 & 50 & 45.4 \\
7 & 60 & 52.6 \\
8 & 70 & n.a. \\
9 & 80 & n.a. \\
10 & 90 & 74.6 \\
11 & 100 & 80.2 \\
12 & 110 & n.a. \\
\hline n.a. $=$ not available.
\end{tabular}

CALCULATIONS FOR SHOT $($ GRADE $=\mathrm{A})$

Slope $=0.8128$

$\mathrm{V}_{\mathrm{I}}=0.38 \mathrm{~km} / \mathrm{s}$

$\mathrm{V}_{2}=3.87 \mathrm{~km} / \mathrm{s}$

Minimum depth to basalt bedrock $=13.63 \mathrm{~m}$ 
TABLE A26. SEISMIC LINE 8. SHOT 2

\begin{tabular}{lll}
\hline Geophone & Distance from Source $(\mathrm{ft})$ & Arrival Time $(\mathrm{ms})$ \\
\hline 1 & 110 & 86.2 \\
2 & 100 & 73.8 \\
3 & 90 & 60.2 \\
4 & 80 & 59.0 \\
5 & 70 & 52.6 \\
6 & 60 & 47.8 \\
7 & 50 & 41.4 \\
8 & 40 & n.a. \\
9 & 30 & n.a. \\
10 & 20 & 14.6 \\
11 & 10 & 4.6 \\
.12 & 0 & 0.0 \\
\hline n.a. =not available. &
\end{tabular}

CALCULATIONS FOR SHOT $($ GRADE $=C$ )

Slope $=0.7514$

$V_{1}=0.41 \mathrm{~km} / \mathrm{s}$

$\mathrm{V}_{2}=3.87 \mathrm{~km} / \mathrm{s}$

Minimum depth to basalt bedrock $=15.82 \mathrm{~m}$

TABLE A27. SEISMIC LINE 8, SHOT 3

\begin{tabular}{lll}
\hline Geophone & Distance from Source $(\mathrm{ft})$ & Arrival Time $(\mathrm{ms})$ \\
\hline 1 & 0 & 0.0 \\
2 & 10 & 19.0 \\
3 & 20 & 16.6 \\
4 & 30 & 24.2 \\
5 & 40 & 29.8 \\
6 & 50 & 30.2 \\
7 & 60 & 33.0 \\
8 & 70 & n.a. \\
9 & 80 & n.a. \\
10 & 90 & 38.2 \\
11 & 100 & 42.2 \\
12 & 110 & n.a. \\
\hline
\end{tabular}

n.a. $=$ not available.

CALCULATIONS FOR SHOT (GRADE=B)

Slope $=0.2048$

$\mathrm{V}_{1}=1.49 \mathrm{~km} / \mathrm{s}$

$\mathrm{V}_{2}=3.87 \mathrm{~km} / \mathrm{s}$

Minimum depth to basalt bedrock $=27.68 \mathrm{~m}$ 
TABLE A28. SEISMIC LINE 8, SHOT 4

\begin{tabular}{lll}
\hline Geophone & Distance from Source $(\mathrm{ft})$ & Arrival Time $(\mathrm{ms})$ \\
\hline $\mathrm{I}$ & 110 & 47.8 \\
2 & 100 & 44.2 \\
3 & 90 & 40.6 \\
4 & 80 & 44.6 \\
5 & 70 & 41.4 \\
6 & 60 & 35.4 \\
7 & 50 & 32.2 \\
8 & 40 & 0.2 \\
9 & 30 & 0.2 \\
10 & 20 & 16.6 \\
11 & 10 & 7.8 \\
12 & 0 & 0.0 \\
\hline
\end{tabular}

\section{CALCULATIONS FOR SHOT (GRADE $=\mathrm{C}$ )}

Slope $=0.4226$

$\mathrm{V}_{1}=0.72 \mathrm{~km} / \mathrm{s}$

$\mathrm{V}_{2}=3.87 \mathrm{~km} / \mathrm{s}$

Minimum depth to basalt bedrock $=14.37 \mathrm{~m}$

TABLE A29. SEISMIC LINE 9, SHOT I

\begin{tabular}{lll}
\hline Geophone & Distance from Source $(\mathrm{ft})$ & Arrival Time $(\mathrm{ms})$ \\
\hline 1 & 0 & 0.0 \\
2 & 10 & 8.2 \\
3 & 20 & 17.0 \\
4 & 30 & 25.0 \\
5 & 40 & 33.0 \\
6 & 50 & 41.0 \\
7 & 60 & 52.6 \\
8 & 70 & n.a. \\
9 & 80 & n.a. \\
10 & 90 & 57.0 \\
11 & 100 & 65.0 \\
12 & 110 & n.a. \\
\hline n.a. $=$ not available. &
\end{tabular}

CALCULATIONS FOR SHOT (GRADE $=\mathrm{B})$

Slope $=0.6239$

$\mathrm{V}_{1}=0.49 \mathrm{~km} / \mathrm{s}$

$\mathrm{V}_{2}=3.87 \mathrm{~km} / \mathrm{s}$

Minimum depth to basalt bedrock $=14.07 \mathrm{~m}$ 
TABLE A30. SEISMIC LINE 9, SHOT 2

\begin{tabular}{lll}
\hline Geophone & Distance from Source $(\mathrm{ft})$ & Arrival Time $(\mathrm{ms})$ \\
\hline 1 & 110 & 61.8 \\
2 & 100 & 57.4 \\
3 & 90 & 66.6 \\
4 & 80 & 61.4 \\
5 & 70 & 58.4 \\
6 & 60 & 54.6 \\
7 & 50 & 44.6 \\
8 & 40 & n.a. \\
9 & 30 & n.a. \\
10 & 20 & 15.4 \\
11 & 10 & 7.4 \\
12 & 0. & 0.0 \\
\hline n.a. $=$ not available. &
\end{tabular}

\section{CALCULATIONS FOR SHOT $($ GRADE $=\mathrm{A})$}

Slope $=0.3900$

$\mathrm{V}_{1}=0.78 \mathrm{~km} / \mathrm{s}$

$\mathrm{V}_{2}=3.87 \mathrm{~km} / \mathrm{s}$

Minimum depth to basalt bedrock $=23.75 \mathrm{~m}$

TABLE A31. SEISMIC LINE 10. SHOT 1

\begin{tabular}{lll}
\hline Geophone & Distance from Source $(\mathrm{ft})$ & Arrival Time $(\mathrm{ms})$ \\
\hline 1 & 0 & 0.0 \\
2 & 10 & 8.2 \\
3 & 20 & 16.6 \\
4 & 30 & 26.6 \\
5 & 40 & 35.0 \\
6 & 50 & 43.0 \\
7 & 60 & 51.0 \\
8 & 70 & n.a. \\
9 & 80 & n.a. \\
10 & 90 & 57.8 \\
11 & 100 & 72.2 \\
12 & 110 & n.a. \\
\hline n.a.=not available. &
\end{tabular}

CALCULATIONS FOR SHOT $($ GRADE $=A$ )

Slope $=0.7413$

$\mathrm{V}_{1}=0.41 \mathrm{~km} / \mathrm{s}$

$\mathrm{V}_{2}=3.87 \mathrm{~km} / \mathrm{s}$

Minimum depth to basalt bedrock $=13.30 \mathrm{~m}$ 
TABLE A32. SEISMIC LINE 10, SHOT 2

\begin{tabular}{lll}
\hline Geophone & Distance from Source $(\mathrm{ft})$ & Arrival Time $(\mathrm{ms})$ \\
\hline 1 & 110 & 81.8 \\
2 & 100 & 77.8 \\
3 & 90 & 70.6 \\
4 & 80 & 56.6 \\
5 & 70 & 47.0 \\
6 & 60 & 33.0 \\
7 & 50 & 41.0 \\
8 & 40 & n.a. \\
9 & 30 & n.a. \\
10 & 20 & 15.0 \\
11 & 10 & 7.0 \\
12 & 0 & 0.0 \\
\hline n.a. $=$ not available. &
\end{tabular}

CALCULATIONS FOR SHOT $(G R A D E=B)$

Slope $=0.7527$

$\mathrm{V}_{\mathrm{l}}=0.41 \mathrm{~km} / \mathrm{s}$

$\mathrm{V}_{2}=3.87 \mathrm{~km} / \mathrm{s}$

Minimum depth to basalt bedrock $=14.89 \mathrm{~m}$

TABLE A33. SEISMIC LINE 11 , SHOT 1

\begin{tabular}{lll}
\hline Geophone & Distance from Source $(\mathrm{ft})$ & Arrival Time $(\mathrm{ms})$ \\
\hline 1 & 0 & 0.0 \\
2 & 10 & 14.2 \\
3 & 20 & 14.2 \\
4 & 30 & 17.4 \\
5 & 40 & 22.6 \\
6 & 50 & 23.0 \\
7 & 60 & 25.0 \\
8 & 70 & n.a. \\
9 & 80 & n.a. \\
10 & 90 & 37.0 \\
11 & 100 & 43.8 \\
12 & 110 & n.a. \\
\hline n.a. =not available &
\end{tabular}

CALCULATIONS FOR SHOT (GRADE=D)

Slope $=0.1200$

$\mathrm{V}_{1}=2.54 \mathrm{~km} / \mathrm{s}$

$\mathrm{V}_{2}=3.87 \mathrm{~km} / \mathrm{s}$

Minimum depth to basalt bedrock $=34.14 \mathrm{~m}$ 
TABLE A34. SEISMIC LINE 11 , SHOT 2

\begin{tabular}{lll}
\hline Geophone & Distance from Source $(\mathrm{ft})$ & Arrival Time $(\mathrm{ms})$ \\
\hline 1 & 110 & 57.0 \\
2 & 100 & 54.2 \\
3 & 90 & 49.0 \\
4 & 80 & 39.0 \\
5 & 70 & 33.8 \\
6 & 60 & 30.2 \\
7 & 50 & 25.8 \\
8 & 40 & n.a. \\
9 & 30 & n.a. \\
10 & 20 & 17.4 \\
11 & 10 & 6.2 \\
12 & 0 & 0.0 \\
\hline n.a. $=$ not available. &
\end{tabular}

\section{CALCULATIONS FOR SHOT (GRADE=B)}

Slope $=0.5219$

$\mathrm{V}_{\mathrm{l}}=0.58 \mathrm{~km} / \mathrm{s}$

$\mathrm{V}_{2}=3.87 \mathrm{~km} / \mathrm{s}$

Minimum depth to basalt bedrock $=14.28 \mathrm{~m}$

TABLE A35. SEISMIC LINE 12, SHOT 1

\begin{tabular}{lll}
\hline Geophone & Distance from Source $(\mathrm{ft})$ & Arrival Time $(\mathrm{ms})$ \\
\hline 1 & 0 & 0.0 \\
2 & 10 & 8.2 \\
3 & 20 & 16.6 \\
4 & 30 & 24.6 \\
5 & 40 & 33.4 \\
6 & 50 & 42.6 \\
7 & 60 & 50.6 \\
8 & 70 & n.a. \\
9 & 80 & n.a. \\
10 & 90 & 66.2 \\
11 & 100 & 68.6 \\
12 & 110 & n.a. \\
\hline
\end{tabular}

n.a.=not available.

\section{CALCULATIONS FOR SHOT $($ GRADE $=\mathrm{A})$}

Slope $=0.2400$

$\mathrm{V}_{1}=1.27 \mathrm{~km} / \mathrm{s}$

$\mathrm{V}_{2}=3.87 \mathrm{~km} / \mathrm{s}$

Minimum depth to basalt bedrock $=40.83 \mathrm{~m}$ 
TABLE A36. SEISMIC LINE 12, SHOT 2

\begin{tabular}{lll}
\hline Geophone & Distance from Source $(\mathrm{ft})$ & Arrival Time $(\mathrm{ms})$ \\
\hline 1 & 110 & 61.0 \\
2 & 100 & 54.2 \\
3 & 90 & 41.8 \\
4 & 80 & 35.8 \\
5 & 70 & 49.0 \\
6 & 60 & 33.8 \\
7 & 50 & 30.2 \\
8 & 40 & n.a. \\
9 & 30 & n.a. \\
10 & 20 & 23.8 \\
11 & 10 & 6.2 \\
12 & 0 & 0.0. \\
\hline n.a.=not available. &
\end{tabular}

\section{CALCULATIONS FOR SHOT $(\mathrm{GRADE}=\mathrm{A})$}

Slope $=0.5451$

$\mathrm{V}_{\mathrm{I}}=0.56 \mathrm{~km} / \mathrm{s}$

$V_{2}=3.87 \mathrm{~km} / \mathrm{s}$

Minimum depth to basalt bedrock $=14.79 \mathrm{~m}$

TABLE A37. SEISMIC LINE 12, SHOT 3

\begin{tabular}{lll}
\hline Geophone & Distance from Source $(\mathrm{ft})$ & Arrival Time $(\mathrm{ms})$ \\
\hline 1 & 0 & 0.0 \\
2 & 10 & 5.8 \\
3 & 20 & 16.6 \\
4 & 30 & 24.2 \\
5 & 40 & 35.4 \\
6 & 50 & 35.8 \\
7 & 60 & 39.0 \\
8 & 70 & n.a. \\
9 & 80 & n.a. \\
10 & 90 & 50.2 \\
11 & 100 & 59.0 \\
12 & 110 & n.a. \\
\hline
\end{tabular}

n.a. =not available.

\section{CALCULATIONS FOR SHOT $(G R A D E=A)$}

Slope $=0.3631$

$\mathrm{V}_{1}=0.84 \mathrm{~km} / \mathrm{s}$

$V_{2}=3.87 \mathrm{~km} / \mathrm{s}$

Minimum depth to basalt bedrock $=18.54 \mathrm{~m}$ 
TABLE A38. SEISMIC LINE 12, SHOT 4

\begin{tabular}{lll}
\hline Geophone & Distance from Source $(\mathrm{ft})$ & Arrival Time $(\mathrm{ms})$ \\
\hline 1 & 110 & 63.0 \\
2 & 100 & 61.8 \\
3 & 90 & 61.8 \\
4 & 80 & 48.6 \\
5 & 70 & 45.8 \\
6 & 60 & 43.4 \\
7 & 50 & 41.8 \\
8 & 40 & n.a. \\
9 & 30 & n.a. \\
10 & 20 & 15.4 \\
11 & 10 & 6.6 \\
12 & 0 & 0.0 \\
\hline
\end{tabular}

n.a. $=$ not available.

\section{CALCULATIONS FOR SHOT (GRADE $=\mathrm{B})$}

Slope $=0.3611$

$\mathrm{V}_{\mathrm{1}}=0.84 \mathrm{~km} / \mathrm{s}$

$\mathrm{V}_{2}=3.87 \mathrm{~km} / \mathrm{s}$

Minimum depth to basalt bedrock $=23.50 \mathrm{~m}$

TABLE A39. SEISMIC LINE 12, SHOT 5

\begin{tabular}{lll}
\hline Geophone & Distance from Source $(\mathrm{ft})$ & Arrival Time $(\mathrm{ms})$ \\
\hline 1 & 0 & 0.0 \\
2 & 10 & 17.4 \\
3 & 20 & n.a. \\
4 & 30 & 27.0 \\
5 & 40 & 34.6 \\
6 & 50 & 39.0 \\
7 & 60 & 43.0 \\
8 & 70 & n.a. \\
9 & 80 & n.a. \\
10 & 90 & 49.0 \\
11 & 100 & 53.4 \\
12 & 110 & n.a. \\
\hline n.a. $=$ not available. &
\end{tabular}

CALCULATIONS FOR SHOT $($ GRADE $=$ A)

Slope $=0.2462$

$\mathrm{V}_{1}=1.24 \mathrm{~km} / \mathrm{s}$

$\mathrm{V}_{2}=3.87 \mathrm{~km} / \mathrm{s}$

Minimum depth to basalt bedrock $=29.75 \mathrm{~m}$ 
TABLE A40. SEISMIC LINE 12, SHOT 6

\begin{tabular}{lll}
\hline Geophone & Distance from Source (ft) & Arrival Time $(\mathrm{ms})$ \\
\hline 1 & 110 & 77.4 \\
2 & 100 & 75.8 \\
3 & 90 & 75.4 \\
4 & 80 & 61.0 \\
5 & 70 & 52.2 \\
6 & 60 & 39.8 \\
7 & 50 & 36.2 \\
8 & 40 & n.a. \\
9 & 30 & n.a. \\
10 & 20 & 17.0 \\
11 & 10 & 7.4 \\
12 & 0 & 0.0 \\
\hline n.a. $=$ not available. &
\end{tabular}

CALCULATIONS FOR SHOT $(G R A D E=B)$

Slope $=0.1000$

$\mathrm{V}_{1}=3.05 \mathrm{~km} / \mathrm{s}$

$\mathrm{V}_{2}=3.87 \mathrm{~km} / \mathrm{s}$

Minimum depth to basalt bedrock $=170.11 \mathrm{~m}$

TABLE A41. SEISMIC LINE 12, SHOT 7

\begin{tabular}{lll}
\hline Geophone & Distance from Source $(\mathrm{ft})$ & Arrival Time $(\mathrm{ms})$ \\
\hline 1 & 0 & 0.0 \\
2 & 10 & 7.8 \\
3 & 20 & 16.6 \\
4 & 30 & 26.2 \\
5 & 40 & 35.0 \\
6 & 50 & 43.0 \\
7 & 60 & 50.6 \\
8 & 70 & n.a. \\
9 & 80 & n.a. \\
10 & 90 & 52.6 \\
11 & 100 & 53.8 \\
12 & 110 & n.a. \\
\hline
\end{tabular}

n.a.=not available.

CALCULATIONS FOR SHOT $($ GRADE $=$ A)

Slope $_{1}=0.8593$, Slope $_{2}=0.0769$

$\mathrm{V}_{1}=0.35 \mathrm{~km} / \mathrm{s}$

$\mathrm{V}_{2}=3.96 \mathrm{~km} / \mathrm{s}$

Depth to basalt bedrock $=8.18 \mathrm{~m}$ 
TABLE A42. SEISMIC LINE 12, SHOT 8

\begin{tabular}{lll}
\hline Geophone & Distance from Source $(\mathrm{ft})$ & Arrival Time $(\mathrm{ms})$ \\
\hline 1 & 110 & 50.6 \\
2 & 100 & 47.8 \\
3 & 90 & 43.8 \\
4 & 80 & 39.4 \\
5 & 70 & 40.6 \\
6 & 60 & 36.6 \\
7 & 50 & 36.2 \\
8 & 40 & n.a. \\
9 & 30 & n.a. \\
10 & 20 & 17.4 \\
11 & 10 & 6.6 \\
12 & 0 & 0.0 \\
\hline n.a. =not available. &
\end{tabular}

CALCULATIONS FOR SHOT (GRADE $=\mathrm{B})$

Slope $=0.2457$

$\mathrm{V}_{1}=1.24 \mathrm{~km} / \mathrm{s}$

$\mathrm{V}_{2}=3.87 \mathrm{~km} / \mathrm{s}$

Minimum depth to basalt bedrock $=27.47 \mathrm{~m}$

TABLE A43. SEISMIC LINE 12, SHOT 10

\begin{tabular}{lll}
\hline Geophone & Distance from Source $(\mathrm{ft})$ & Arrival Time $(\mathrm{ms})$ \\
\hline 1 & 0 & 0.0 \\
2 & 10 & 15.8 \\
3 & 20 & 21.4 \\
4 & 30 & 29.8 \\
5 & 40 & 35.4 \\
6 & 50 & 39.4 \\
7 & 60 & 42.2 \\
8 & 70 & n.a. \\
9 & 80 & n.a. \\
10 & 90 & 50.6 \\
11 & 100 & 51.0 \\
12 & 110 & n.a. \\
\hline
\end{tabular}

n.a. $=$ not available.

CALCULATIONS FOR SHOT (GRADE $=B$ )

Slope $=0.2447$

$\mathrm{V}_{1}=1.25 \mathrm{~km} / \mathrm{s}$

$\mathrm{V}_{2}=3.87 \mathrm{~km} / \mathrm{s}$

Minimum depth to basalt bedrock $=28.37 \mathrm{~m}$ 
TABLE A44. SEISMIC LINE 12, SHOT 11

\begin{tabular}{lll}
\hline Geophone & Distance from Source $(\mathrm{ft})$ & Arrival Time $(\mathrm{ms})$ \\
\hline 1 & 110 & 57.4 \\
2 & 100 & 47.4 \\
3 & 90 & 45.4 \\
4 & 80 & 43.0 \\
5 & 70 & 41.8 \\
6 & 60 & 36.6 \\
7 & 50 & 35.8 \\
8 & 40 & n.a. \\
9 & 30 & n.a. \\
10 & 20 & 26.6 \\
11 & 10 & 9.0 \\
12 & 0 & 0.0 \\
\hline n.a.=not available. &
\end{tabular}

CALCULATIONS FOR SHOT (GRADE $=\mathrm{A})$

Slope $=0.2621$

$\mathrm{V}_{\mathrm{I}}=1.16 \mathrm{~km} / \mathrm{s}$

$\mathrm{V}_{2}=3.87 \mathrm{~km} / \mathrm{s}$

Minimum depth to basalt bedrock $=24.10 \mathrm{~m}$

TABLE A45. SEISMIC LINE 12, SHOT 12

\begin{tabular}{lll}
\hline Geophone & Distance from Source $(\mathrm{ft})$ & Arrival Time $(\mathrm{ms})$ \\
\hline 1 & 0 & 0.0 \\
2 & 10 & 7.8 \\
3 & 20 & 16.6 \\
4 & 30 & 24.2 \\
5 & 40 & 34.6 \\
6 & 50 & 38.2 \\
7 & 60 & 40.6 \\
8 & 70 & n.a. \\
9 & 80 & n.a. \\
10 & 90 & 44.6 \\
11 & 100 & 47.0 \\
12 & 110 & n.a. \\
\hline n.a. $=$ not available. & \\
\end{tabular}

\section{CALCULATIONS FOR SHOT (GRADE=B)}

Slope $=0.1881$

$\mathrm{V}_{1}=1.62 \mathrm{~km} / \mathrm{s}$

$\mathrm{V}_{2}=3.87 \mathrm{~km} / \mathrm{s}$

Minimum depth to basalt bedrock $=34.91 \mathrm{~m}$ 
TABLE A46. SEISMIC LINE 12, SHOT 13

\begin{tabular}{lll}
\hline Geophone & Distance from Source $(\mathrm{ft})$ & Arrival Time $(\mathrm{ms})$ \\
\hline 1 & 110 & 47.8 \\
2 & 100 & 45.8 \\
3 & 90 & 40.6 \\
4 & 80 & 40.6 \\
5 & 70 & 39.0 \\
6 & 60 & 38.6 \\
7 & 50 & 37.8 \\
8 & 40 & n.a. \\
9 & 30 & n.a. \\
10 & 20 & 16.2 \\
11 & 10 & 7.0 \\
12 & 0 & 0.0 \\
\hline n.a. $=$ not available.
\end{tabular}

CALCULATIONS FOR SHOT (GRADE $=\mathrm{B})$

Slope $_{1}=0.7600$, Slope $_{2}=0.0686$

$\mathrm{V}_{1}=0.40 \mathrm{~km} / \mathrm{s}$

$V_{2}=4.44 \mathrm{~km} / \mathrm{s}$

Depth to basalt bedrock $=6.92 \mathrm{~m}$

TABLE A47. SEISMIC LINE 12, SHOT 14

\begin{tabular}{lll}
\hline Geophone & Distance from Source $(\mathrm{ft})$ & Arrival Time $(\mathrm{ms})$ \\
\hline 1 & 0 & 0.0 \\
2 & 10 & 6.6 \\
3 & 20 & 22.6 \\
4 & 30 & 24.6 \\
5 & 40 & 25.4 \\
6 & 50 & 32.2 \\
7 & 60 & 35.0 \\
8 & 70 & n.a. \\
9 & 80 & n.a. \\
10 & 90 & 35.0 \\
11 & 100 & 35.8 \\
12 & 110 & n.a. \\
\hline n.a. $=$ not available. &
\end{tabular}

CALCULATIONS FOR SHOT (GRADE $=\mathrm{B})$

Slope $=0.1702$

$\mathrm{V}_{1}=1.79 \mathrm{~km} / \mathrm{s}$

$\mathrm{V}_{2}=3.87 \mathrm{~km} / \mathrm{s}$

Minimum depth to basalt bedrock $=28.21 \mathrm{~m}$ 
TABLE A48. SEISMIC LINE 12, SHOT 15

\begin{tabular}{lll}
\hline Geophone & Distance from Source $(\mathrm{ft})$ & Arrival Time $(\mathrm{ms})$ \\
\hline 1 & 110 & 44.6 \\
2 & 100 & 40.2 \\
3 & 90 & 35.4 \\
4 & 80 & 35.8 \\
5 & 70 & 34.2 \\
6 & 60 & 31.4 \\
7 & 50 & 31.0 \\
8 & 40 & n.a. \\
9 & 30 & n.a. \\
10 & 20 & 15.0 \\
11 & 10 & 16.6 \\
12 & 0 & 0.0 \\
\hline n.a. $=$ not available. &
\end{tabular}

\section{CALCULATIONS FOR SHOT (GRADE $=\mathrm{B})$}

Slope $=0.2251$

$\mathrm{V}_{1}=1.35 \mathrm{~km} / \mathrm{s}$

$\mathrm{V}_{2}=3.87 \mathrm{~km} / \mathrm{s}$

Minimum depth to basalt bedrock $=25.98 \mathrm{~m}$

TABLE A49. SEISMIC LINE 12, SHOT 16

\begin{tabular}{lll}
\hline Geophone & Distance from Source $(\mathrm{ft})$ & Arrival Time $(\mathrm{ms})$ \\
\hline 1 & 0 & 0.0 \\
2 & 10 & 8.6 \\
3 & 20 & 16.6 \\
4 & 30 & 30.2 \\
5 & 40 & 37.4 \\
6 & 50 & 32.6 \\
7 & 60 & 33.4 \\
8 & 70 & n.a. \\
9 & 80 & n.a. \\
10 & 90 & 49.0 \\
11 & 100 & 52.2 \\
12 & 110 & n.a. \\
\hline n.a. =not available. &
\end{tabular}

n.a. $=$ not available.

\section{CALCULATIONS FOR SHOT (GRADE $=\mathrm{B})$}

Slope $=0.4259$

$\mathrm{V}_{\mathrm{l}}=0.72 \mathrm{~km} / \mathrm{s}$

$\mathrm{V}_{2}=3.87 \mathrm{~km} / \mathrm{s}$

Minimum depth to basalt bedrock $=16.14 \mathrm{~m}$ 
TABLE A50. SEISMIC LINE 12, SHOT 17

\begin{tabular}{lll}
\hline Geophone & Distance from Source $(\mathrm{ft})$ & Arrival Time $(\mathrm{ms})$ \\
\hline 1 & 110 & 53.4 \\
2 & 100 & 37.0 \\
3 & 90 & 35.4 \\
4 & 80 & 34.6 \\
5 & 70 & 32.2 \\
6 & 60 & 31.0 \\
7 & 50 & 30.2 \\
8 & 40 & n.a. \\
9 & 30 & n.a. \\
10 & 20 & 16.6 \\
11 & 10 & 7.8 \\
12 & 0 & 0.0 \\
\hline n.a.=not available.
\end{tabular}

\section{CALCULATIONS FOR SHOT (GRADE $=$ A)}

Slope $=0.1417$

$\mathrm{V}_{1}=2.15 \mathrm{~km} / \mathrm{s}$

$\mathrm{V}_{2}=3.87 \mathrm{~km} / \mathrm{s}$

Minimum depth to basalt bedrock $=37.69 \mathrm{~m}$

TABLE A51. SEISMIC LINE 12, SHOT 18

\begin{tabular}{lll}
\hline Geophone & Distance from Source $(\mathrm{ft})$ & Arrival Time $(\mathrm{ms})$ \\
\hline 1 & 0 & 0.0 \\
2 & 10 & 7.8 \\
3 & 20 & 15.4 \\
4 & 30 & 24.2 \\
5 & 40 & 29.4 \\
6 & 50 & 30.2 \\
7 & 60 & 31.4 \\
8 & 70 & n.a. \\
9 & 80 & n.a. \\
10 & 90 & 35.0 \\
11 & 100 & 41.4 \\
12 & 110 & n.a. \\
\hline
\end{tabular}

n.a. $=$ not available.

\section{CALCULATIONS FOR SHOT (GRADE $=$ A)}

Slope $=0.1143$

$\mathrm{V}_{1}=2.67 \mathrm{~km} / \mathrm{s}$

$\mathrm{V}_{2}=3.87 \mathrm{~km} / \mathrm{s}$

Minimum depth to basalt bedrock $=51.37 \mathrm{~m}$ 
TABLE A52. SEISMIC LINE 12, SHOT 19

\begin{tabular}{lll}
\hline Geophone & Distance from Source $(\mathrm{ft})$ & Arrival Time $(\mathrm{ms})$ \\
\hline 1 & 110 & 37.4 \\
2 & 100 & 35.8 \\
3 & 90 & 33.8 \\
4 & 80 & 32.6 \\
5 & 70 & 31.8 \\
6 & 60 & 31.4 \\
7 & 50 & 29.0 \\
8 & 40 & n.a. \\
9 & 30 & n.a. \\
10 & 20 & 21.4 \\
11 & 10 & 6.2 \\
12 & 0 & 0.0 \\
\hline n.a. $=$ not available. &
\end{tabular}

CALCULATIONS FOR SHOT (GRADE $=B$ )

Slope $=0.1639$

$\mathrm{V}_{1}=1.86 \mathrm{~km} / \mathrm{s}$

$\mathrm{V}_{2}=3.87 \mathrm{~km} / \mathrm{s}$

Minimum depth to basalt bedrock $=30.48 \mathrm{~m}$

TABLE A53. SEISMIC LINE 12, SHOT 20

\begin{tabular}{lll}
\hline Geophone & Distance from Source $(\mathrm{ft})$ & Arrival Time $(\mathrm{ms})$ \\
\hline 1 & 0 & 0.0 \\
2 & 10 & 7.4 \\
3 & 20 & 23.0 \\
4 & 30 & 28.6 \\
5 & 40 & 35.8 \\
6 & 50 & 39.4 \\
7 & 60 & 40.6 \\
8 & 70 & n.a. \\
9 & 80 & n.a. \\
10 & 90 & 39.8 \\
11 & 100 & 41.0 \\
12 & 110 & n.a. \\
\hline n.a. $=$ not available. &
\end{tabular}

n.a. $=$ not available.

CALCULATIONS FOR SHOT (GRADE $=\mathrm{B})$

Slope $_{1}=0.9280$, Slope $_{2}=0.0845$

$\mathrm{V}_{\mathrm{l}}=0.33 \mathrm{~km} / \mathrm{s}$

$\mathrm{V}_{2}=3.61 \mathrm{~km} / \mathrm{s}$

Depth to basalt bedrock $=5.34 \mathrm{~m}$ 
TABLE A54. SEISMIC LINE 12, SHOT 21

\begin{tabular}{lll}
\hline Geophone & Distance from Source $(\mathrm{ft})$ & Arrival Time $(\mathrm{ms})$ \\
\hline 1 & 110 & 37.8 \\
2 & 100 & 37.0 \\
3 & 90 & 36.6 \\
4 & 80 & 35.0 \\
5 & 70 & 33.4 \\
6 & 60 & 32.6 \\
7 & 50 & 31.0 \\
8 & 40 & n.a. \\
9 & 30 & n.a. \\
10 & 20 & 16.6 \\
11 & 10 & 7.8 \\
12 & 0 & 0.0 \\
\hline n.a.=not available. &
\end{tabular}

\section{CALCULATIONS FOR SHOT $($ GRADE $=$ A)}

Slope $=0.1157$

$\mathrm{V}_{1}=2.64 \mathrm{~km} / \mathrm{s}$

$\mathrm{V}_{2}=3.87 \mathrm{~km} / \mathrm{s}$

Minimum depth to basalt bedrock $=52.41 \mathrm{~m}$

TABLE A55. SEISMIC LINE 12, SHOT 22

\begin{tabular}{lll}
\hline Geophone & Distance from Source $(\mathrm{ft})$ & Arrival Time $(\mathrm{ms})$ \\
\hline 1 & 0 & 0.0 \\
2 & 10 & 1.4 \\
3 & 20 & 16.6 \\
4 & 30 & 25.0 \\
5 & 40 & 29.4 \\
6 & 50 & 33.0 \\
7 & 60 & 42.2 \\
8 & 70 & n.a. \\
9 & 80 & n.a. \\
10 & 90 & 45.8 \\
11 & 100 & 46.6 \\
12 & 110 & n.a. \\
\hline
\end{tabular}

n.a.=not available.

\section{CALCULATIONS FOR SHOT $($ GRADE $=$ A)}

Slope $=0.3120$

$\mathrm{V}_{1}=0.98 \mathrm{~km} / \mathrm{s}$

$\mathrm{V}_{2}=3.87 \mathrm{~km} / \mathrm{s}$

Minimum depth to basalt bedrock $=19.55 \mathrm{~m}$ 
TABLE A56. SEISMIC LINE 12. SHOT 23

\begin{tabular}{lll}
\hline Geophone & Distance from Source $(\mathrm{ft})$ & Arrival Time $(\mathrm{ms})$ \\
\hline 1 & 110 & 39.8 \\
2 & 100 & 43.4 \\
3 & 90 & 39.0 \\
4 & 80 & 35.8 \\
5 & 70 & 31.4 \\
6 & 60 & 28.6 \\
7 & 50 & 25.8 \\
8 & 40 & n.a. \\
9 & 30 & n.a. \\
10 & 20 & 25.0 \\
11 & 10 & 7.8 \\
12 & 0 & 0.0 \\
\hline n.a.=not available. &
\end{tabular}

CALCULATIONS FOR SHOT (GRADE $=$ A)

Slope $=0.3531$

$\mathrm{V}_{1}=0.86 \mathrm{~km} / \mathrm{s}$

$\mathrm{V}_{2}=3.87 \mathrm{~km} / \mathrm{s}$

Minimum depth to basalt bedrock $=15.73 \mathrm{~m}$

TABLE A57. SEISMIC LINE 12. SHOT 24

\begin{tabular}{lll}
\hline Geophone & Distance from Source $(\mathrm{ft})$ & Arrival Time $(\mathrm{ms})$ \\
\hline 1 & 0 & 0.0 \\
2 & 10 & 6.6 \\
3 & 20 & 15.8 \\
4 & 30 & 21.4 \\
5 & 40 & 23.8 \\
6 & 50 & 26.2 \\
7 & 60 & 28.6 \\
8 & 70 & n.a. \\
9 & 80 & n.a. \\
10 & 90 & 29.8 \\
11 & 100 & 31.0 \\
12 & 110 & n.a. \\
\hline
\end{tabular}

n.a. $=$ not available.

CALCULATIONS FOR SHOT $($ GRADE $=\mathrm{C})$

Slope $=0.1252$

$\mathrm{V}_{1}=2.44 \mathrm{~km} / \mathrm{s}$

$\mathrm{V}_{2}=3.87 \mathrm{~km} / \mathrm{s}$

Minimum depth to basalt bedrock $=36.22 \mathrm{~m}$ 
TABLE A58. SEISMIC LINE 12, SHOT 25

\begin{tabular}{lll}
\hline Geophone & Distance from Source $(\mathrm{ft})$ & Arrival Time $(\mathrm{ms})$ \\
\hline 1 & 110 & 36.6 \\
2 & 100 & 34.6 \\
3 & 90 & 33.0 \\
4 & 80 & 23.4 \\
5 & 70 & 27.0 \\
6 & 60 & 26.2 \\
7 & 50 & 23.0 \\
8 & 40 & n.a. \\
9 & 30 & n.a. \\
10 & 20 & 15.4 \\
11 & 10 & 5.8 \\
12 & 0 & 0.0 \\
\hline n.a. $=$ not available. &
\end{tabular}

\section{CALCULATIONS FOR SHOT (GRADE $=\mathrm{A})$}

Slope $=0.2086$

$\mathrm{V}_{\mathrm{1}}=1.46 \mathrm{~km} / \mathrm{s}$

$\mathrm{V}_{2}=3.87 \mathrm{~km} / \mathrm{s}$

Minimum depth to basalt bedrock $=22.05 \mathrm{~m}$

TABLE A59. SEISMIC LINE 12, SHOT 26

\begin{tabular}{lll}
\hline Geophone & Distance from Source $(\mathrm{ft})$ & Arrival Time $(\mathrm{ms})$ \\
\hline 1 & 0 & 0.0 \\
2 & 10 & 6.6 \\
3 & 20 & 21.8 \\
4 & 30 & 25.8 \\
5 & 40 & 28.6 \\
6 & 50 & 29.4 \\
7 & 60 & 29.4 \\
8 & 70 & n.a. \\
9 & 80 & n.a. \\
10 & 90 & 31.4 \\
11 & 100 & 39.0 \\
12 & 110 & n.a. \\
\hline
\end{tabular}

n.a. =not available.

\section{CALCULATIONS FOR SHOT $($ GRADE $=\mathrm{A})$}

Slope $=0.1833$

$\mathrm{V}_{1}=1.66 \mathrm{~km} / \mathrm{s}$

$\mathrm{V}_{2}=3.87 \mathrm{~km} / \mathrm{s}$

Minimum depth to basalt bedrock $=28.66 \mathrm{~m}$ 
TABLE A60. SEISMIC LINE 12, SHOT 27

\begin{tabular}{lll}
\hline Geophone & Distance from Source $(\mathrm{ft})$ & Arrival Time $(\mathrm{ms})$ \\
\hline 1 & 110 & 48.6 \\
2 & 100 & 45.8 \\
3 & 90 & 43.4 \\
4 & 80 & 41.0 \\
5 & 70 & 38.2 \\
6 & 60 & 37.4 \\
7 & 50 & 32.6 \\
8 & 40 & n.a. \\
9 & 30 & n.a. \\
10 & 20 & 15.4 \\
11 & 10 & 4.6 \\
12 & 0 & 0.0 \\
\hline
\end{tabular}

n.a. $=$ not available.

\section{CALCULATIONS FOR SHOT (GRADE=B)}

Slope $=0.2320$

$\mathrm{V}_{1}=1.31 \mathrm{~km} / \mathrm{s}$

$\mathrm{V}_{2}=3.87 \mathrm{~km} / \mathrm{s}$

Minimum depth to basalt bedrock $=27.90 \mathrm{~m}$

TABLE A61. SEISMIC LINE 12, SHOT 28

\begin{tabular}{lll}
\hline Geophone & Distance from Source $(\mathrm{ft})$ & Arrival Time $(\mathrm{ms})$ \\
\hline 1 & 0 & 0.0 \\
2 & 10 & 12.6 \\
3 & 20 & 17.8 \\
4 & 30 & 19.4 \\
5 & 40 & 19.0 \\
6 & 50 & 21.4 \\
7 & 60 & 27.0 \\
8 & 70 & n.a. \\
9 & 80 & n.a. \\
10 & 90 & 32.6 \\
11 & 100 & 34.6 \\
12 & 110 & n.a. \\
\hline n.a. $=$ not available. &
\end{tabular}

CALCULATIONS FOR SHOT $($ GRADE $=B$ )

Slope $=0.2136$

$\mathrm{V}_{1}=1.43 \mathrm{~km} / \mathrm{s}$

$\mathrm{V}_{2}=3.87 \mathrm{~km} / \mathrm{s}$

Minimum depth to basalt bedrock $=20.52 \mathrm{~m}$ 
TABLE A62. SEISMIC LINE 12, SHOT 29

\begin{tabular}{lll}
\hline Geophone & Distance from Source $(\mathrm{ft})$ & Arrival Time $(\mathrm{ms})$ \\
\hline 1 & 110 & 39.0 \\
2 & 100 & 32.2 \\
3 & 90 & 35.8 \\
4 & 80 & 31.8 \\
5 & 70 & 28.6 \\
6 & 60 & 27.8 \\
7 & 50 & 28.6 \\
8 & 40 & n.a. \\
9 & 30 & n.a. \\
10 & 20 & 17.8 \\
11 & 10 & 9.4 \\
12 & 0 & 0.0 \\
\hline n.a. $=$ not available. &
\end{tabular}

CALCULATIONS FOR SHOT (GRADE $=\mathrm{A})$

Slope $=0.1743$

$\mathrm{V}_{1}=1.75 \mathrm{~km} / \mathrm{s}$

$\mathrm{V}_{2}=3.87 \mathrm{~km} / \mathrm{s}$

Minimum depth to basalt bedrock $=29.74 \mathrm{~m}$

TABLE A63. SEISMIC LINE 13, SHOT I

\begin{tabular}{lll}
\hline Geophone & Distance from Source $(\mathrm{ft})$ & Arrival Time $(\mathrm{ms})$ \\
\hline 1 & 10 & 6.2 \\
2 & 20 & 15.4 \\
3 & 30 & 18.6 \\
4 & 40 & 21.4 \\
5 & 50 & 25.0 \\
6 & 60 & 27.0 \\
7 & 70 & 29.4 \\
8 & 80 & n.a. \\
9 & 90 & n.a. \\
10 & 100 & 36.6 \\
11 & 110 & 38.6 \\
12 & 120 & n.a. \\
\hline n.a.=not available &
\end{tabular}

n.a. $=$ not available.

\section{CALCULATIONS FOR SHOT $($ GRADE $=\mathrm{A})$}

Slope $=0.2550$

$\mathrm{V}_{1}=1.20 \mathrm{~km} / \mathrm{s}$

$\mathrm{V}_{2}=3.87 \mathrm{~km} / \mathrm{s}$

Minimum depth to basalt bedrock $=18.82 \mathrm{~m}$ 
TABLE A64. SEISMIC LINE 13. SHOT 2

\begin{tabular}{lll}
\hline Geophone & Distance from Source $(\mathrm{ft})$ & Arrival Time $(\mathrm{ms})$ \\
\hline 1 & 120 & 41.0 \\
2 & 110 & 38.2 \\
3 & 100 & 39.4 \\
4 & 90 & 35.8 \\
5 & 80 & 35.4 \\
6 & 70 & 34.2 \\
7 & 60 & 33.0 \\
8 & 50 & n.a. \\
9 & 40 & n.a. \\
10 & 30 & 27.4 \\
11 & 20 & 19.4 \\
12 & 10 & 14.6 \\
\hline n.a.=not available: &
\end{tabular}

CALCULATIONS FOR SHOT (GRADE $=\mathrm{B})$

Slope $=0.1427$

$\mathrm{V}_{1}=2.14 \mathrm{~km} / \mathrm{s}$

$\mathrm{V}_{2}=3.87 \mathrm{~km} / \mathrm{s}$

Minimum depth to basalt bedrock $=40.42 \mathrm{~m}$

TABLE A65. SEISMIC LINE 13, SHOT 3

\begin{tabular}{lll}
\hline Geophone & Distance from Source $(\mathrm{ft})$ & Arrival Time $(\mathrm{ms})$ \\
\hline 1 & 10 & 9.4 \\
2 & 20 & 17.8 \\
3 & 30 & 23.8 \\
4 & 40 & 26.2 \\
5 & 50 & 25.8 \\
6 & 60 & 27.0 \\
7 & 70 & 28.2 \\
8 & 80 & n.a. \\
9 & 90 & n.a. \\
10 & 100 & 33.0 \\
11 & 110 & 34.2 \\
12 & 120 & n.a. \\
\hline n.a. =not available &
\end{tabular}

\section{CALCULATIONS FOR SHOT $($ GRADE $=A)$}

Slope $=0.1442$

$\mathrm{V}_{\mathrm{I}}=2.11 \mathrm{~km} / \mathrm{s}$

$\mathrm{V}_{2}=3.87 \mathrm{~km} / \mathrm{s}$

Minimum depth to basalt bedrock $=32.23 \mathrm{~m}$ 
TABLE A66. SEISMIC LINE 13, SHOT 4

\begin{tabular}{lll}
\hline Geophone & Distance from Source $(\mathrm{ft})$ & Arrival Time $(\mathrm{ms})$ \\
\hline 1 & 120 & 37.4 \\
2 & 110 & 36.2 \\
3 & 100 & 35.0 \\
4 & 90 & 34.2 \\
5 & 80 & 33.0 \\
6 & 70 & 31.0 \\
7 & 60 & 29.0 \\
8 & 50 & n.a. \\
9 & 40 & n.a. \\
10 & 30 & 19.4 \\
11 & 20 & 17.4 \\
L2 & 10 & 15.4 \\
\hline n.a. $=$ not available. &
\end{tabular}

CALCULATIONS FOR SHOT (GRADE $=\mathrm{B})$

Slope $=0.2114$

$\mathrm{V}_{\mathrm{1}}=1.44 \mathrm{~km} / \mathrm{s}$

$\mathrm{V}_{2}=3.87 \mathrm{~km} / \mathrm{s}$

Minimum depth to basalt bedrock $=21.72 \mathrm{~m}$

TABLE A67. SEISMIC LINE 14, SHOT 1

\begin{tabular}{lll}
\hline Geophone & Distance from Source (ft) & Arrival Time $(\mathrm{ms})$ \\
\hline 1 & 10 & 7.0 \\
2 & 20 & 13.0 \\
3 & 30 & 14.6 \\
4 & 40 & 15.0 \\
5 & 50 & 19.8 \\
6 & 60 & 25.4 \\
7 & 70 & 26.2 \\
8 & 80 & n.a. \\
9 & 90 & n.a. \\
10 & 100 & n.a. \\
11 & 110 & n.a. \\
12 & 120 & n.a. \\
\hline n.a. $=$ not available. &
\end{tabular}

\section{CALCULATIONS FOR SHOT $(\mathrm{GRADE}=\mathrm{C})$}

Slope $_{1}=0.2949$

$\mathrm{V}_{1}=1.03 \mathrm{~km} / \mathrm{s}$

$\mathrm{V}_{2}=3.87 \mathrm{~km} / \mathrm{s}$

Minimum depth to basalt bedrock $=11.10 \mathrm{~m}$ 
TABLE A68. SEISMIC LINE 14. SHOT 2

\begin{tabular}{lll}
\hline Geophone & Distance from Source $(\mathrm{ft})$ & Arrival Time $(\mathrm{ms})$ \\
\hline 1 & 120 & n.a. \\
2 & 110 & n.a. \\
3 & 100 & 29.0 \\
4 & 90 & 28.2 \\
5 & 80 & 27.4 \\
6 & 70 & 22.6 \\
7 & 60 & 19.0 \\
8 & 50 & n.a. \\
9 & 40 & n.a. \\
10 & 30 & 16.6 \\
11 & 20 & 10.2 \\
12 & 10 & 8.2 \\
\hline n.a.-not available. &
\end{tabular}

CALCULATIONS FOR SHOT $($ GRADE $=C)$

Slope $_{1}=0.2804$, Slope $_{2}=0.0800$

$\mathrm{V}_{\mathrm{l}}=1.09 \mathrm{~km} / \mathrm{s}$

$\mathrm{V}_{2}=3.81 \mathrm{~km} / \mathrm{s}$

Depth to basalt bedrock $=11.91 \mathrm{~m}$

TABLE A69. SEISMIC LINE 14. SHOT 3

\begin{tabular}{lll}
\hline Geophone & Distance from Source $(\mathrm{ft})$ & Arrival Time $(\mathrm{ms})$ \\
\hline 1 & 10 & 7.4 \\
2 & 20 & 10.6 \\
3 & 30 & 13.0 \\
4 & 40 & 15.0 \\
5 & 50 & 16.6 \\
6 & 60 & 19.8 \\
7 & 70 & 23.8 \\
8 & 80 & n.a. \\
9 & 90 & n.a. \\
10 & 100 & 27.8 \\
11 & 110 & 35.8 \\
12 & 120 & n.a. \\
\hline n.a. $=$ not available. &
\end{tabular}

CALCULATIONS FOR SHOT (GRADE=B)

Slope $=0.2777$

$\mathrm{V}_{1}=1.10 \mathrm{~km} / \mathrm{s}$

$\mathrm{V}_{2}=3.87 \mathrm{~km} / \mathrm{s}$

Minimum depth to basalt bedrock $=15.53 \mathrm{~m}$ 
TABLE A70. SEISMIC LINE 14. SHOT 4

\begin{tabular}{lll}
\hline Geophone & Distance from Source $(\mathrm{ft})$ & Arrival Time $(\mathrm{ms})$ \\
\hline 1 & 120 & 35.0 \\
2 & 110 & 30.6 \\
3 & 100 & 28.2 \\
4 & 90 & 23.8 \\
5 & 80 & 24.2 \\
6 & 70 & 23.8 \\
7 & 60 & 24.2 \\
8 & 50 & n.a. \\
9 & 40 & n.a. \\
10 & 30 & 21.8 \\
11 & 20 & 16.6 \\
12 & 10 & 7.8 \\
\hline n.a. $=$ not available. &
\end{tabular}

\section{CALCULATIONS FOR SHOT (GRADE=B)}

Slope $=0.1514$

$\mathrm{V}_{\mathrm{1}}=2.01 \mathrm{~km} / \mathrm{s}$

$\mathrm{V}_{2}=3.87 \mathrm{~km} / \mathrm{s}$

Minimum depth to basalt bedrock $=30.12 \mathrm{~m}$

TABLE A71. SEISMIC LINE 15. SHOT I

\begin{tabular}{lll}
\hline Geophone & Distance from Source $(\mathrm{ft})$ & Arrival Time $(\mathrm{ms})$ \\
\hline 1 & 10 & 5.4 \\
2 & 20 & 12.2 \\
3 & 30 & 22.2 \\
4 & 40 & 25.4 \\
5 & 50 & 27.8 \\
6 & 60 & 30.6 \\
7 & 70 & n.a. \\
8 & 80 & n.a. \\
9 & 90 & n.a. \\
10 & 100 & 43.0 \\
11 & 110 & 47.4 \\
12 & 120 & n.a. \\
\hline
\end{tabular}

n.a. $=$ not available.

CALCULATIONS FOR SHOT (GRADE=A)

Slope $=0.3095$

$\mathrm{V}_{\mathrm{I}}=0.99 \mathrm{~km} / \mathrm{s}$

$\mathrm{V}_{2}=3.87 \mathrm{~km} / \mathrm{s}$

Minimum depth to basalt bedrock $=19.73 \mathrm{~m}$ 
TABLE A72. SEISMIC LINE 15, SHOT 2

\begin{tabular}{lll}
\hline Geophone & Distance from Source $(\mathrm{ft})$ & Arrival Time $(\mathrm{ms})$ \\
\hline 1 & 120 & 58.6 \\
2 & 110 & 55.0 \\
3 & 100 & 54.2 \\
4 & 90 & 49.4 \\
5 & 80 & 45.4 \\
6 & 70 & 42.2 \\
7 & 60 & 43.8 \\
8 & 50 & n.a. \\
9 & 40 & n.a. \\
10 & 30 & 20.2 \\
11 & 20 & 15.4 \\
12 & 10 & 11.4 \\
\hline n.a. $=$ not available. &
\end{tabular}

CALCULATIONS FOR SHOT $(\mathrm{GRADE}=\mathrm{B})$

Slope $=0.4452$

$\mathrm{V}_{1}=0.68 \mathrm{~km} / \mathrm{s}$

$V_{2}=3.87 \mathrm{~km} / \mathrm{s}$

Minimum depth to basalt bedrock $=17.10 \mathrm{~m}$

TABLE A73. SEISMIC LINE 15, SHOT 3

\begin{tabular}{lll}
\hline Geophone & Distance from Source $(\mathrm{ft})$ & Arrival Time $(\mathrm{ms})$ \\
\hline $\mathrm{l}$ & 10 & 7.0 \\
2 & 20 & 14.2 \\
3 & 30 & 21.4 \\
4 & 40 & 24.6 \\
5 & 50 & 27.4 \\
6 & 60 & 29.4 \\
7 & 70 & 29.8 \\
8 & 80 & n.a. \\
9 & 90 & n.a. \\
10 & 100 & 43.4 \\
11 & 110 & n.a. \\
12 & 120 & n.a. \\
\hline
\end{tabular}

n.a. $=$ not available.

\section{CALCULATIONS FOR SHOT $($ GRADE $=C)$}

Slope $=0.4040$

$\mathrm{V}_{1}=0.75 \mathrm{~km} / \mathrm{s}$

$\mathrm{V}_{2}=3.87 \mathrm{~km} / \mathrm{s}$

Minimum depth to basalt bedrock $=13.67 \mathrm{~m}$ 
TABLE A74. SEISMIC LINE 15, SHOT 4

\begin{tabular}{lll}
\hline Geophone & Distance from Source ( $\mathrm{ft})$ & Arrival Time $(\mathrm{ms})$ \\
\hline 1 & 120 & 53.8 \\
2 & 110 & 50.2 \\
3 & 100 & 48.6 \\
4 & 90 & 46.2 \\
5 & 80 & 48.6 \\
6 & 70 & 39.8 \\
7 & 60 & 36.2 \\
8 & 50 & n.a. \\
9 & 40 & n.a. \\
10 & 30 & 21.4 \\
11 & 20 & 15.0 \\
12 & 10 & 8.2 \\
\hline
\end{tabular}

n.a. $=$ not available.

\section{CALCULATIONS FOR SHOT (GRADE $=\mathrm{B})$}

Slope $=0.4335$

$\mathrm{V}_{1}=0.70 \mathrm{~km} / \mathrm{s}$

$\mathrm{V}_{2}=3.87 \mathrm{~km} / \mathrm{s}$

Minimum depth to basalt bedrock $=15.86 \mathrm{~m}$

TABLE A75. SEISMIC LINE 16, SHOT 1

\begin{tabular}{lll}
\hline Geophone & Distance from Source $(\mathrm{ft})$ & Arrival Time $(\mathrm{ms})$ \\
\hline $\mathrm{l}$ & 0 & 0.0 \\
2 & 10 & 11.0 \\
3 & 20 & 25.4 \\
4 & 30 & 41.8 \\
5 & 40 & 46.2 \\
6 & 50 & 49.4 \\
7 & 60 & n.a. \\
8 & 70 & n.a. \\
9 & 80 & n.a. \\
10 & 90 & n.a. \\
11 & 100 & n.a. \\
12 & 110 & n.a. \\
\hline n.a.=not available. &
\end{tabular}

CALCULATIONS FOR SHOT $($ GRADE $=\mathrm{A})$

Slope $=0.3800$

$\mathrm{V}_{1}=0.80 \mathrm{~km} / \mathrm{s}$

$\mathrm{V}_{2}=3.87 \mathrm{~km} / \mathrm{s}$

Minimum depth to basalt bedrock $=18.64 \mathrm{~m}$ 
TABLE A76. SEISMIC LINE 16, SHOT 2

\begin{tabular}{lll}
\hline Geophone & Distance from Source (ft) & Arrival Time $(\mathrm{ms})$ \\
\hline 1 & 110 & n.a. \\
2 & 100 & n.a. \\
3 & 90 & 75.4 \\
4 & 80 & 73.0 \\
5 & 70 & 70.2 \\
6 & 60 & 61.4 \\
7 & 50 & 57.4 \\
8 & 40 & n.a. \\
9 & 30 & n.a. \\
10 & 20 & 42.2 \\
11 & 10 & n.a. \\
12 & 0 & .0 .0 \\
\hline n.a.=not available. &
\end{tabular}

\section{CALCULATIONS FOR SHOT (GRADE=B)}

Slope $=0.4760$

$\mathrm{V}_{1}=0.64 \mathrm{~km} / \mathrm{s}$

$\mathrm{V}_{2}=3.87 \mathrm{~km} / \mathrm{s}$

Minimum depth to basalt bedrock $=22.18 \mathrm{~m}$

TABLE A77. SEISMIC LINE 16, SHOT 3

\begin{tabular}{lll}
\hline Geophone & Distance from Source $(\mathrm{ft})$ & Arrival Time $(\mathrm{ms})$ \\
\hline 1 & 0 & 0.0 \\
2 & 10 & 31.0 \\
3 & 20 & 38.2 \\
4 & 30 & 44.2 \\
5 & 40 & 53.8 \\
6 & 50 & n.a. \\
7 & 60 & n.a. \\
8 & 70 & n.a. \\
9 & 80 & n.a. \\
10 & 90 & n.a. \\
11 & 100 & n.a. \\
12 & 110 & n.a. \\
\hline n.a. $=$ not available. &
\end{tabular}

\section{CALCULATIONS FOR SHOT $($ GRADE $=\mathrm{A})$}

Slope $=0.7440$

$\mathrm{V}_{1}=0.41 \mathrm{~km} / \mathrm{s}$

$\mathrm{V}_{2}=3.87 \mathrm{~km} / \mathrm{s}$

Minimum depth to basalt bedrock $=10.44 \mathrm{~m}$ 
TABLE A78. SEISMIC LINE 16, SHOT 4

\begin{tabular}{lll}
\hline Geophone & Distance from Source $(\mathrm{ft})$ & Arrival Time $(\mathrm{ms})$ \\
\hline 1 & 110 & n.a. \\
2 & 100 & n.a. \\
3 & 90 & n.a. \\
4 & 80 & n.a. \\
5 & 70 & 80.2 \\
6 & 60 & 73.0 \\
7 & 50 & 68.6 \\
8 & 40 & n.a. \\
9 & 30 & n.a. \\
10 & 20 & 49.4 \\
11 & 10 & n.a. \\
12 & 0 & 0.0 \\
\hline n.a.=not available. &
\end{tabular}

\section{CALCULATIONS FOR SHOT $($ GRADE $=A)$}

Slope $=0.6086$

$\mathrm{V}_{1}=0.50 \mathrm{~km} / \mathrm{s}$

$\mathrm{V}_{2}=3.87 \mathrm{~km} / \mathrm{s}$

Minimum depth to basalt bedrock $=18.87 \mathrm{~m}$

TABLE A79. SEISMIC LINE 17, SHOT I

\begin{tabular}{lll}
\hline Geophone & Distance from Source $(\mathrm{ft})$ & Arrival Time $(\mathrm{ms})$ \\
\hline 1 & 10 & 5.4 \\
2 & 20 & 9.4 \\
3 & 30 & 17.0 \\
4 & 40 & 21.8 \\
5 & 50 & 26.6 \\
6 & 60 & 27.8 \\
7 & 70 & 30.2 \\
8 & 80 & n.a. \\
9 & 90 & n.a. \\
10 & 100 & 39.8 \\
11 & 110 & 44.6 \\
12 & 120 & n.a. \\
\hline
\end{tabular}

n.a. $=$ not available.

CALCULATIONS FOR SHOT $($ GRADE $=B$ )

Slope $=0.3900$

$\mathrm{V}_{1}=0.78 \mathrm{~km} / \mathrm{s}$

$\mathrm{V}_{2}=3.87 \mathrm{~km} / \mathrm{s}$

Minimum depth to basalt bedrock $=14.34 \mathrm{~m}$ 
TABLE A80. SEISMIC LINE 17, SHOT 2

\begin{tabular}{lll}
\hline Geophone & Distance from Source $(\mathrm{ft})$ & Arrival Time $(\mathrm{ms})$ \\
\hline 1 & 120 & n.a. \\
2 & 110 & n.a. \\
3 & 100 & 54.6 \\
4 & 90 & 53.4 \\
5 & 80 & 52.2 \\
6 & 70 & 50.6 \\
7 & 60 & 45.0 \\
8 & 50 & n.a. \\
9 & 40 & n.a. \\
10 & 30 & 27.0 \\
11 & 20 & 17.4 \\
12 & 10 & 13.8 \\
\hline n.a. $=$ not available. & \\
\hline
\end{tabular}

CALCULATIONS FOR SHOT (GRADE $=$ B)

Slope $=0.1320$

$\mathrm{V}_{1}=2.31 \mathrm{~km} / \mathrm{s}$

$\mathrm{V}_{2}=3.87 \mathrm{~km} / \mathrm{s}$

Minimum depth to basalt bedrock $=67.25 \mathrm{~m}$

TABLE A81. SEISMIC LINE 17, SHOT 3

\begin{tabular}{lll}
\hline Geophone & Distance from Source $(\mathrm{ft})$ & Arrival Time $(\mathrm{ms})$ \\
\hline 1 & 10 & 4.6 \\
2 & 20 & 13.0 \\
3 & 30 & 22.6 \\
4 & 40 & 29.4 \\
5 & 50 & 39.8 \\
6 & 60 & 49.8 \\
7 & 70 & 55.4 \\
8 & 80 & n.a. \\
9 & 90 & n.a. \\
10 & 100 & 77.4 \\
11 & 110 & 82.6 \\
12 & 120 & n.a. \\
\hline n.a. =not available. &
\end{tabular}

CALCULATIONS FOR SHOT $(\mathrm{GRADE}=\mathrm{A})$

Slope $=0.7847$

$\mathrm{V}_{\mathrm{1}}=0.39 \mathrm{~km} / \mathrm{s}$

$V_{2}=3.87 \mathrm{~km} / \mathrm{s}$

Minimum depth to basalt bedrock $=14.44 \mathrm{~m}$ 
TABLE A82. SEISMIC LINE 17, SHOT 4

\begin{tabular}{lll}
\hline Geophone & Distance from Source $(\mathrm{ft})$ & Arrival Time $(\mathrm{ms})$ \\
\hline 1 & 120 & n.a. \\
2 & 110 & 94.2 \\
3 & 100 & 84.2 \\
4 & 90 & 73.8 \\
5 & 80 & 61.8 \\
6 & 70 & 56.6 \\
7 & 60 & 47.4 \\
8 & 50 & n.a. \\
9 & 40 & n.a. \\
10 & 30 & 29.0 \\
11 & 20 & 13.4 \\
12 & 10 & n.a. \\
\hline n.a.=not available. &
\end{tabular}

CALCULATIONS FOR SHOT $(G R A D E=A)$

Slope $=0.8364$

$V_{1}=0.36 \mathrm{~km} / \mathrm{s}$

$\mathrm{V}_{2}=3.87 \mathrm{~km} / \mathrm{s}$

Minimum depth to basalt bedrock $=15.66 \mathrm{~m}$

TABLE A83. SEISMIC LINE 18, SHOT I

\begin{tabular}{lll}
\hline Geophone & Distance from Source $(\mathrm{ft})$ & Arrival Time $(\mathrm{ms})$ \\
\hline 1 & 10 & 6.2 \\
2 & 20 & 15.4 \\
3 & 30 & 24.6 \\
4 & 40 & 30.2 \\
5 & 50 & 32.6 \\
6 & 60 & 35.0 \\
7 & 70 & 43.4 \\
8 & 80 & n.a. \\
9 & 90 & n.a. \\
10 & 100 & 61.4 \\
11 & 110 & 64.6 \\
12 & 120 & n.a. \\
\hline n.a. $=$ not available. &
\end{tabular}

CALCULATIONS FOR SHOT $(G R A D E=B)$

Slope $=0.5768$

$\mathrm{V}_{\mathrm{l}}=0.53 \mathrm{~km} / \mathrm{s}$

$\mathrm{V}_{2}=3.87 \mathrm{~km} / \mathrm{s}$

Minimum depth to basalt bedrock $=14.92 \mathrm{~m}$ 
TABLE A84. SEISMIC LINE 18, SHOT 2

\begin{tabular}{lll}
\hline Geophone & Distance from Source (ft) & Arrival Time (ms) \\
\hline 1 & 120 & 62.2 \\
2 & 110 & 59.4 \\
3 & 100 & 55.0 \\
4 & 90 & 55.0 \\
5 & 80 & 53.8 \\
6 & 70 & 50.6 \\
7 & 60 & n.a. \\
8 & 50 & n.a. \\
9 & 40 & n.a. \\
10 & 30 & n.a. \\
11 & 20 & n.a. \\
12 & 10 & n.a. \\
\hline
\end{tabular}

n.a. $=$ not available.

CALCULATIONS FOR SHOT $(G R A D E=B)$

Slope $=0.2137$

$\mathrm{V}_{1}=1.43 \mathrm{~km} / \mathrm{s}$

$\mathrm{V}_{2}=3.87 \mathrm{~km} / \mathrm{s}$

Minimum depth to basalt bedrock $=40.48 \mathrm{~m}$

TABLE A85. SEISMIC LINE 18, SHOT 3

\begin{tabular}{lll}
\hline Geophone & Distance from Source $(\mathrm{ft})$ & Arrival Time $(\mathrm{ms})$ \\
\hline 1 & 10 & 3.4 \\
2 & 20 & 14.2 \\
3 & 30 & 21.4 \\
4 & 40 & 30.6 \\
5 & 50 & 35.8 \\
6 & 60 & 42.6 \\
7 & 70 & 45.4 \\
8 & 80 & n.a. \\
9 & 90 & n.a. \\
10 & 100 & 59.0 \\
11 & 110 & 60.6 \\
12 & 120 & n.a. \\
\hline n.a. =not available. &
\end{tabular}

CALCULATIONS FOR SHOT $($ GRADE $=C)$

Slope $=0.5738$

$\mathrm{V}_{\mathrm{I}}=0.53 \mathrm{~km} / \mathrm{s}$

$\mathrm{V}_{2}=3.87 \mathrm{~km} / \mathrm{s}$

Minimum depth to basalt bedrock $=13.93 \mathrm{~m}$ 
TABLE A86. SEISMIC LINE 18, SHOT 4

\begin{tabular}{lll}
\hline Geophone & Distance from Source $(\mathrm{ft})$ & Arrival Time $(\mathrm{ms})$ \\
\hline 1 & 120 & n.a. \\
2 & 110 & n.a. \\
3 & 100 & 71.4 \\
4 & 90 & 67.0 \\
5 & 80 & 50.2 \\
6 & 70 & 52.6 \\
7 & 60 & n.a. \\
8 & 50 & n.a. \\
9 & 40 & n.a. \\
10 & 30 & n.a. \\
11 & 20 & n.a. \\
12 & 10 & n.a. \\
n.a. $=$ not available. &
\end{tabular}

\section{CALCULATIONS FOR SHOT $($ GRADE $=\mathrm{A})$}

Slope $=0.7274$

$\mathrm{V}_{1}=0.42 \mathrm{~km} / \mathrm{s}$

$\mathrm{V}_{2}=3.87 \mathrm{~km} / \mathrm{s}$

Minimum depth to basalt bedrock $=13.39 \mathrm{~m}$

TABLE A87. SEISMIC LINE 18, SHOT 5

\begin{tabular}{lll}
\hline Geophone & Distance from Source $(\mathrm{ft})$ & Arrival Time $(\mathrm{ms})$ \\
\hline 1 & 10 & 6.2 \\
2 & 20 & 15.4 \\
3 & 30 & 24.2 \\
4 & 40 & 30.2 \\
5 & 50 & 37.0 \\
6 & 60 & 44.6 \\
7 & 70 & 47.8 \\
8 & 80 & n.a. \\
9 & 90 & n.a. \\
10 & 100 & n.a. \\
11 & 110 & 49.0 \\
12 & 120 & n.a. \\
\hline
\end{tabular}

n.a. $=$ not available.

\section{CALCULATIONS FOR SHOT (GRADE $=$ A)}

Slope $=0.7112$

$\mathrm{V}_{\mathrm{1}}=0.43 \mathrm{~km} / \mathrm{s}$

$\mathrm{V}_{2}=3.87 \mathrm{~km} / \mathrm{s}$

Minimum depth to basalt bedrock $=29.91 \mathrm{~m}$ 
TABLE A88. SEISMIC LINE 18, SHOT 6

\begin{tabular}{lll}
\hline Geophone & Distance from Source $(\mathrm{ft})$ & Arrival Time $(\mathrm{ms})$ \\
\hline 1 & 120 & n.a. \\
2 & 110 & n.a. \\
3 & 100 & 55.8 \\
4 & 90 & 52.2 \\
5 & 80 & 50.6 \\
6 & 70 & 47.0 \\
7 & 60 & 37.0 \\
8 & 50 & n.a. \\
9 & 40 & n.a. \\
10 & 30 & 25.4 \\
11 & 20 & 16.6 \\
12 & 10 & n.a. \\
\hline n.a.=not available. &
\end{tabular}

CALCULATIONS FOR SHOT (GRADE $=\mathrm{A})$

Slope $=0.2800$

$V_{1}=1.09 \mathrm{~km} / \mathrm{s}$

$\mathrm{V}_{2}=3.87 \mathrm{~km} / \mathrm{s}$

Minimum depth to basalt bedrock $=89.18 \mathrm{~m}$

TABLE A89. SEISMIC LINE 18, SHOT 7

\begin{tabular}{lll}
\hline Geophone & Distance from Source $(\mathrm{ft})$ & Arrival Time $(\mathrm{ms})$ \\
\hline 1 & 10 & 6.2 \\
2 & 20 & 15.4 \\
3 & 30 & 24.6 \\
4 & 40 & 34.2 \\
5 & 50 & 36.6 \\
6 & 60 & 39.4 \\
7 & 70 & 48.2 \\
8 & 80 & n.a. \\
9 & 90 & n.a. \\
10 & 100 & n.a. \\
11 & 110 & n.a. \\
12 & 120 & n.a. \\
\hline n.a. =not available. &
\end{tabular}

CALCULATIONS FOR SHOT (GRADE=B)

Slope $=0.4667$

$\mathrm{V}_{1}=0.65 \mathrm{~km} / \mathrm{s}$

$\mathrm{V}_{2}=3.87 \mathrm{~km} / \mathrm{s}$

Minimum depth to basalt bedrock $=46.40 \mathrm{~m}$ 
TABLE A90. SEISMIC LINE 18, SHOT 8

\begin{tabular}{lll}
\hline Geophone & Distance from Source $(\mathrm{ft})$ & Arrival Time $(\mathrm{ms})$ \\
\hline $\mathrm{l}$ & 120 & n.a. \\
2 & 110 & n.a. \\
3 & 100 & 45.4 \\
4 & 90 & 40.6 \\
5 & 80 & 37.8 \\
6 & 70 & 35.8 \\
7 & 60 & 35.0 \\
8 & 50 & n.a. \\
9 & 40 & n.a. \\
10 & 30 & 23.0 \\
11 & 20 & 16.2 \\
12 & 10 & n.a. \\
\hline n.a.=not available. &
\end{tabular}

CALCULATIONS FOR SHOT (GRADE=B)

Slope $=0.2600$

$\mathrm{V}_{1}=1.17 \mathrm{~km} / \mathrm{s}$

$\mathrm{V}_{2}=3.87 \mathrm{~km} / \mathrm{s}$

Minimum depth to basalt bedrock $=23.08 \mathrm{~m}$

TABLE A91. SEISMIC LINE 18, SHOT 9

\begin{tabular}{lll}
\hline Geophone & Distance from Source $(\mathrm{ft})$ & Arrival Time $(\mathrm{ms})$ \\
\hline 1 & 10 & 7.0 \\
2 & 20 & 15.8 \\
3 & 30 & 25.4 \\
4 & 40 & 33.8 \\
5 & 50 & 40.2 \\
6 & 60 & 42.2 \\
7 & 70 & 44.2 \\
8 & 80 & n.a. \\
9 & 90 & n.a. \\
10 & 100 & 49.0 \\
11 & 110 & 50.2 \\
12 & 120 & n.a. \\
\hline n.a. $=$ not available. & &
\end{tabular}

CALCULATIONS FOR SHOT (GRADE=A)

Slope $=0.1663$

$\mathrm{V}_{1}=1.83 \mathrm{~km} / \mathrm{s}$

$\mathrm{V}_{2}=3.87 \mathrm{~km} / \mathrm{s}$

Minimum depth to basalt bedrock $=43.23 \mathrm{~m}$ 
TABLE A92. SEISMIC LINE 18, SHOT 10

\begin{tabular}{lll}
\hline Geophone & Distance from Source $(\mathrm{ft})$ & Arrival Time $(\mathrm{ms})$ \\
\hline 1 & 120 & n.a. \\
2 & 110 & n.a. \\
3 & 100 & 45.4 \\
4 & 90 & 43.0 \\
5 & 80 & 41.8 \\
6 & 70 & 37.8 \\
7 & 60 & n.a. \\
8 & 50 & n.a. \\
9 & 40 & n.a. \\
10 & 30 & 25.0 \\
11 & 20 & 15.8 \\
12 & 10 & 13.4 \\
\hline
\end{tabular}

n.a. =not available.

\section{CALCULATIONS FOR SHOT (GRADE $=\mathrm{A})$}

Slope $=0.2973$

$\mathrm{V}_{1}=1.03 \mathrm{~km} / \mathrm{s}$

$\mathrm{V}_{2}=3.87 \mathrm{~km} / \mathrm{s}$

Minimum depth to basalt bedrock $=19.95 \mathrm{~m}$

TABLE A93. SEISMIC LINE 18. SHOT 11

\begin{tabular}{lll}
\hline Geophone & Distance from Source $(\mathrm{ft})$ & Arrival Time $(\mathrm{ms})$ \\
\hline 1 & 10 & 7.8 \\
2 & 20 & 17.0 \\
3 & 30 & 26.2 \\
4 & 40 & 35.0 \\
5 & 50 & 43.4 \\
6 & 60 & 48.6 \\
7 & 70 & 51.4 \\
8 & 80 & n.a. \\
9 & 90 & n.a. \\
10 & 100 & n.a. \\
11 & 110 & n.a. \\
12 & 120 & n.a. \\
\hline n.a. $=$ not available. & \\
\end{tabular}

\section{CALCULATIONS FOR SHOT (GRADE $=\mathrm{A})$}

Slope $=0.2800$

$\mathrm{V}_{1}=1.09 \mathrm{~km} / \mathrm{s}$

$\mathrm{V}_{2}=3.87 \mathrm{~km} / \mathrm{s}$

Minimum depth to basalt bedrock $=26.03 \mathrm{~m}$ 
TABLE A94. SEISMIC LINE 18, SHOT 12

\begin{tabular}{lll}
\hline Geophone & Distance from Source $(\mathrm{ft})$ & Arrival Time $(\mathrm{ms})$ \\
\hline 1 & 120 & n.a. \\
2 & 110 & 47.4 \\
3 & 100 & 43.8 \\
4 & 90 & 42.2 \\
5 & 80 & 39.0 \\
6 & 70 & 36.6 \\
7 & 60 & 35.0 \\
8 & 50 & n.a. \\
9 & 40 & n.a. \\
10 & 30 & 27.0 \\
11 & 20 & 23.8 \\
12 & 10 & 19.8 \\
\hline n.a.=not available. &
\end{tabular}

\section{CALCULATIONS FOR SHOT $(G R A D E=A)$}

Slope $=0.2628$

$\mathrm{V}_{1}=1.16 \mathrm{~km} / \mathrm{s}$

$\mathrm{V}_{2}=3.87 \mathrm{~km} / \mathrm{s}$

Minimum depth to basalt bedrock $=23.55 \mathrm{~m}$

TABLE A95. SEISMIC LINE 18, SHOT 13

\begin{tabular}{lll}
\hline Geophone & Distance from Source $(\mathrm{ft})$ & Arrival Time $(\mathrm{ms})$ \\
\hline $\mathrm{l}$ & 10 & 7.0 \\
2 & 20 & 16.6 \\
3 & 30 & 25.8 \\
4 & 40 & 31.8 \\
5 & 50 & 35.8 \\
6 & 60 & 37.8 \\
7 & 70 & 41.8 \\
8 & 80 & n.a. \\
9 & 90 & n.a. \\
10 & 100 & 37.8 \\
11 & 110 & 41.0 \\
12 & 120 & n.a. \\
\hline
\end{tabular}

n.a. $=$ not available.

CALCULATIONS FOR SHOT $($ GRADE $=\mathrm{A})$

Slope $=0.3200$

$\mathrm{V}_{1}=0.95 \mathrm{~km} / \mathrm{s}$

$\mathrm{V}_{2}=3.87 \mathrm{~km} / \mathrm{s}$

Minimum depth to basalt bedrock $=17.83 \mathrm{~m}$ 
TABLE A96. SEISMIC LINE 18, SHOT 14

\begin{tabular}{lll}
\hline Geophone & Distance from Source $(\mathrm{ft})$ & Arrival Time $(\mathrm{ms})$ \\
\hline 1 & 120 & 36.2 \\
2 & 110 & 36.6 \\
3 & 100 & 35.8 \\
4 & 90 & 36.6 \\
5 & 80 & 36.2 \\
6 & 70 & 35.4 \\
7 & 60 & 32.6 \\
8 & 50 & n.a. \\
9 & 40 & n.a. \\
10 & 30 & 24.6 \\
11 & 20 & 15.4 \\
12 & 10 & 7.0 \\
\hline n.a. $=$ not available. &
\end{tabular}

\section{CALCULATIONS FOR SHOT $($ GRADE $=C)$}

Slope $=0.2429$

$\mathrm{V}_{\mathrm{I}}=1.26 \mathrm{~km} / \mathrm{s}$

$\mathrm{V}_{2}=3.87 \mathrm{~km} / \mathrm{s}$

Minimum depth to basalt bedrock $=19.84 \mathrm{~m}$

TABLE A97. SEISMIC LINE 18, SHOT 15

\begin{tabular}{lll}
\hline Geophone & Distance from Source $(\mathrm{ft})$ & Arrival Time $(\mathrm{ms})$ \\
\hline 1 & 10 & 6.2 \\
2 & 20 & 15.8 \\
3 & 30 & 25.0 \\
4 & 40 & 33.4 \\
5 & 50 & 35.8 \\
6 & 60 & 35.8 \\
7 & 70 & 38.2 \\
8 & 80 & n.a. \\
9 & 90 & n.a. \\
10 & 100 & 39.4 \\
11 & 110 & 41.4 \\
12 & 120 & n.a. \\
\hline n.a. $=$ not available. & \\
\end{tabular}

CALCULATIONS FOR SHOT $($ GRADE $=B)$

Slope $=0.1001$

$\mathrm{V}_{\mathrm{I}}=3.05 \mathrm{~km} / \mathrm{s}$

$\mathrm{V}_{2}=3.87 \mathrm{~km} / \mathrm{s}$

Minimum depth to basalt bedrock $=80.80 \mathrm{~m}$ 
TABLE A98. SEISMIC LINE 18, SHOT 16

\begin{tabular}{lll}
\hline Geophone & Distance from Source $(\mathrm{ft})$ & Arrival Time $(\mathrm{ms})$ \\
\hline 1 & 120 & n.a. \\
2 & 110 & n.a. \\
3 & 100 & n.a. \\
4 & 90 & n.a. \\
5 & 80 & n.a. \\
6 & 70 & 35.8 \\
7 & 60 & n.a. \\
8 & 50 & n.a. \\
9 & 40 & n.a. \\
10 & 30 & n.a. \\
11 & 20 & 15.0 \\
12 & 10 & 13.8 \\
\hline
\end{tabular}

n.a. $=$ not available.

\section{CALCULATIONS FOR SHOT $(\mathrm{GRADE}=\mathrm{C})$}

Slope $=0.3826$

$\mathrm{V}_{1}=0.80 \mathrm{~km} / \mathrm{s}$

$\mathrm{V}_{2}=3.87 \mathrm{~km} / \mathrm{s}$

Minimum depth to basalt bedrock $=12.33 \mathrm{~m}$

TABLE A99. SEISMIC LINE 18, SHOT 17

\begin{tabular}{lll}
\hline Geophone & Distance from Source $(\mathrm{ft})$ & Arrival Time $(\mathrm{ms})$ \\
\hline 1 & 10 & 5.8 \\
2 & 20 & 13.4 \\
3 & 30 & 28.2 \\
4 & 40 & 29.0 \\
5 & 50 & 31.4 \\
6 & 60 & 34.6 \\
7 & 70 & 35.4 \\
8 & 80 & n.a. \\
9 & 90 & n.a. \\
10 & 100 & 37.0 \\
11 & 110 & 37.8 \\
12 & 120 & n.a. \\
\hline
\end{tabular}

n.a. $=$ not available.

\section{CALCULATIONS FOR SHOT (GRADE $=\mathrm{C})$}

Slope $=0.1284$

$\mathrm{V}_{\mathrm{I}}=2.37 \mathrm{~km} / \mathrm{s}$

$\mathrm{V}_{2}=3.87 \mathrm{~km} / \mathrm{s}$

Minimum depth to basalt bedrock $=43.80 \mathrm{~m}$ 
TABLE A100. SEISMIC LINE 18, SHOT 18

\begin{tabular}{lll}
\hline Geophone & Distance from Source $(\mathrm{ft})$ & Arrival Time $(\mathrm{ms})$ \\
\hline 1 & 120 & 49.0 \\
2 & 110 & 46.2 \\
3 & 100 & 41.0 \\
4 & 90 & 34.6 \\
5 & 80 & 31.4 \\
6 & 70 & 26.2 \\
7 & 60 & 23.4 \\
8 & 50 & n.a. \\
9 & 40 & n.a. \\
10 & 30 & 11.4 \\
11 & 20 & 8.2 \\
12 & 10 & n.a. \\
\hline
\end{tabular}

n.a. $=$ not available.

CALCULATIONS FOR SHOT $($ GRADE $=\mathrm{A})$

Slope $=0.4105$

$V_{1}=0.74 \mathrm{~km} / \mathrm{s}$

$\mathrm{V}_{2}=3.87 \mathrm{~km} / \mathrm{s}$

Minimum depth to basalt bedrock $=14.96 \mathrm{~m}$

TABLE A101. SEISMIC LINE 19, SHOT 1

\begin{tabular}{lll}
\hline Geophone & Distance from Source $(\mathrm{ft})$ & Arrival Time $(\mathrm{ms})$ \\
\hline 1 & 10 & n.a. \\
2 & 20 & 20.2 \\
3 & 30 & 32.2 \\
4 & 40 & 33.8 \\
5 & 50 & n.a. \\
6 & 60 & n.a. \\
7 & 70 & n.a. \\
8 & 80 & n.a. \\
9 & 90 & n.a. \\
10 & 100 & n.a. \\
11 & 110 & n.a. \\
12 & 120 & n.a. \\
\hline
\end{tabular}

n.a. =not available.

CALCULATIONS FOR SHOT $(G R A D E=D)$

Slope $=0.1600$

$\mathrm{V}_{1}=1.91 \mathrm{~km} / \mathrm{s}$

$\mathrm{V}_{2}=3.87 \mathrm{~km} / \mathrm{s}$

Minimum depth to basalt bedrock $=33.55 \mathrm{~m}$ 
TABLE A 102. SEISMIC LINE 20, SHOT 1

\begin{tabular}{lll}
\hline Geophone & Distance from Source $(\mathrm{ft})$ & Arrival Time $(\mathrm{ms})$ \\
\hline 1 & 10 & 5.8 \\
2 & 20 & 13.4 \\
3 & 30 & 15.0 \\
4 & 40 & 13.4 \\
5 & 50 & 17.4 \\
6 & 60 & n.a. \\
7 & 70 & 19.8 \\
8 & 80 & n.a. \\
9 & 90 & n.a. \\
10 & 100 & 31.4 \\
11 & 110 & 33.4 \\
12 & 120 & n.a. \\
\hline
\end{tabular}

n.a. $=$ not available.

\section{CALCULATIONS FOR SHOT $($ GRADE $=\mathrm{A})$}

Slope $=0.2268$

$\mathrm{V}_{\mathrm{I}}=1.34 \mathrm{~km} / \mathrm{s}$

$V_{2}=3.87 \mathrm{~km} / \mathrm{s}$

Minimum depth to basalt bedrock $=17.73 \mathrm{~m}$

TABLE A 103. SEISMIC LINE 20, SHOT 2

\begin{tabular}{lll}
\hline Geophone & Distance from Source $(\mathrm{ft})$ & Arrival Time $(\mathrm{ms})$ \\
\hline 1 & 120 & 43.4 \\
2 & 110 & 36.6 \\
3 & 100 & 35.8 \\
4 & 90 & 34.2 \\
5 & 80 & 32.2 \\
6 & 70 & n.a. \\
7 & 60 & 30.2 \\
8 & 50 & n.a. \\
9 & 40 & n.a. \\
10 & 30 & 22.6 \\
11 & 20 & 17.8 \\
12 & 10 & 13.8 \\
\hline n.a.=not available. &
\end{tabular}

\section{CALCULATIONS FOR SHOT (GRADE=B)}

Slope $=0.2200$

$\mathrm{V}_{1}=1.39 \mathrm{~km} / \mathrm{s}$

$\mathrm{V}_{2}=3.87 \mathrm{~km} / \mathrm{s}$

Minimum depth to basalt bedrock $=25.19 \mathrm{~m}$ 
TABLE A 104. SEISMIC LINE 20, SHOT 3

\begin{tabular}{lll}
\hline Geophone & Distance from Source $(\mathrm{ft})$ & Arrival Time $(\mathrm{ms})$ \\
\hline 1 & 10 & 6.2 \\
2 & 20 & 10.2 \\
3 & 30 & 18.2 \\
4 & 40 & 25.8 \\
5 & 50 & 27.4 \\
6 & 60 & 32.2 \\
7 & 70 & 34.6 \\
8 & 80 & n.a. \\
9 & 90 & n.a. \\
10 & 100 & n.a. \\
11 & 110 & n.a. \\
12 & 120 & n.a. \\
\hline n.a.=not available. &
\end{tabular}

CALCULATIONS FOR SHOT (GRADE $=$ B)

Slope $=0.3120$

$\mathrm{V}_{1}=0.98 \mathrm{~km} / \mathrm{s}$

$\mathrm{V}_{2}=3.87 \mathrm{~km} / \mathrm{s}$

Minimum depth to basalt bedrock $=14.69 \mathrm{~m}$

TABLE A105. SEISMIC LINE 20. SHOT 4

\begin{tabular}{lll}
\hline Geophone & Distance from Source $(\mathrm{ft})$ & Arrival Time $(\mathrm{ms})$ \\
\hline 1 & 120 & 47.0 \\
2 & 110 & 46.2 \\
3 & 100 & 45.8 \\
4 & 90 & 45.4 \\
5 & 80 & 45.0 \\
6 & 70 & 42.2 \\
7 & 60 & 40.2 \\
8 & 50 & n.a. \\
9 & 40 & n.a. \\
10 & 30 & n.a. \\
11 & 20 & 13.4 \\
12 & 10 & 13.4 \\
\hline n.a. $=$ not available &
\end{tabular}

CALCULATIONS FOR SHOT $($ GRADE $=D)$

Slope $=0.3384$

$\mathrm{V}_{\mathrm{l}}=0.90 \mathrm{~km} / \mathrm{s}$

$\mathrm{V}_{2}=3.87 \mathrm{~km} / \mathrm{s}$

Minimum depth to basalt bedrock $=17.39 \mathrm{~m}$ 
TABLE A 106. SEISMIC LINE 20, SHOT 5

\begin{tabular}{lll}
\hline Geophone & Distance from Source $(\mathrm{ft})$ & Arrival Time $(\mathrm{ms})$ \\
\hline 1 & 10 & 7.0 \\
2 & 20 & 16.6 \\
3 & 30 & 24.6 \\
4 & 40 & 29.8 \\
5 & 50 & 33.4 \\
6 & 60 & 37.4 \\
7 & 70 & 40.6 \\
8 & 80 & n.a. \\
9 & 90 & n.a. \\
10 & 100 & 49.4 \\
11 & 110 & 49.8 \\
12 & 120 & n.a. \\
\hline
\end{tabular}

n.a. $=$ not available.

CALCULATIONS FOR SHOT (GRADE=B)

Slope $=0.3146$

$\mathrm{V}_{1}=0.97 \mathrm{~km} / \mathrm{s}$

$\mathrm{V}_{2}=3.87 \mathrm{~km} / \mathrm{s}$

Minimum depth to basalt bedrock $=20.59 \mathrm{~m}$

TABLE A 107. SEISMIC LINE 20. SHOT 6

\begin{tabular}{lll}
\hline Geophone & Distance from Source $(\mathrm{ft})$ & Arrival Time $(\mathrm{ms})$ \\
\hline 1 & 120 & 55.0 \\
2 & 110 & 53.8 \\
3 & 100 & 51.0 \\
4 & 90 & 50.2 \\
5 & 80 & 48.2 \\
6 & 70 & 45.4 \\
7 & 60 & 43.0 \\
8 & 50 & n.a. \\
9 & 40 & n.a. \\
10 & 30 & n.a. \\
11 & 20 & n.a. \\
12 & 10 & n.a. \\
\hline n.a. $=$ not available. &
\end{tabular}

\section{CALCULATIONS FOR SHOT $($ GRADE $=\mathrm{A})$}

Slope $=0.1986$

$V_{1}=1.54 \mathrm{~km} / \mathrm{s}$

$\mathrm{V}_{2}=3.87 \mathrm{~km} / \mathrm{s}$

Minimum depth to basalt bedrock $=38.08 \mathrm{~m}$ 
TABLE A 108. SEISMIC LINE 20, SHOT 7

\begin{tabular}{lll}
\hline Geophone & Distance from Source $(\mathrm{ft})$ & Arrival Time $(\mathrm{ms})$ \\
\hline 1 & 10 & 8.2 \\
2 & 20 & 21.8 \\
3 & 30 & 31.8 \\
4 & 40 & 37.8 \\
5 & 50 & 39.4 \\
6 & 60 & 41.8 \\
7 & 70 & n.a. \\
8 & 80 & n.a. \\
9 & 90 & n.a. \\
10 & 100 & n.a. \\
11 & 110 & 49.0 \\
12 & 120 & n.a. \\
\hline n.a.=not available. &. &
\end{tabular}

CALCULATIONS FOR SHOT $($ GRADE $=\mathbf{A})$

Slope $=0.1586$

$V_{1}=1.92 \mathrm{~km} / \mathrm{s}$

$\mathrm{V}_{2}=3.87 \mathrm{~km} / \mathrm{s}$

Minimum depth to basalt bedrock $=44.67 \mathrm{~m}$

TABLE A 109. SEISMIC LINE 20, SHOT 8

\begin{tabular}{lll}
\hline Geophone & Distance from Source (ft) & Arrival Time $(\mathrm{ms})$ \\
\hline 1 & 120 & n.a. \\
2 & 110 & n.a. \\
3 & 100 & 47.4 \\
4 & 90 & 45.8 \\
5 & 80 & 41.4 \\
6 & 70 & 39.0 \\
7 & 60 & n.a. \\
8 & 50 & n.a. \\
9 & 40 & n.a. \\
10 & 30 & n.a. \\
11 & 20 & 16.2 \\
12 & 10 & n.a. \\
\hline n.a.=not available. & \\
\multicolumn{2}{l}{}
\end{tabular}

CALCULATIONS FOR SHOT $($ GRADE $=A)$

Slope $=0.2960$

$\mathrm{V}_{\mathrm{l}}=1.03 \mathrm{~km} / \mathrm{s}$

$\mathrm{V}_{2}=3.87 \mathrm{~km} / \mathrm{s}$

Minimum depth to basalt bedrock $=21.12 \mathrm{~m}$ 
TABLE A1 10. SEISMIC LINE 20. SHOT 9

\begin{tabular}{lll}
\hline Geophone & Distance from Source $(\mathrm{ft})$ & Arrival Time $(\mathrm{ms})$ \\
\hline 1 & 10 & 6.6 \\
2 & 20 & 16.2 \\
3 & 30 & 32.2 \\
4 & 40 & 35.4 \\
5 & 50 & 43.8 \\
6 & 60 & 48.6 \\
7 & 70 & 51.4 \\
8 & 80 & n.a. \\
9 & 90 & n.a. \\
10 & 100 & 63.0 \\
11 & 110 & 68.6 \\
12 & 120 & n.a. \\
\hline n.a. $=$ not available. &
\end{tabular}

\section{CALCULATIONS FOR SHOT (GRADE $=$ A)}

Slope $=0.4453$

$\mathrm{V}_{1}=0.68 \mathrm{~km} / \mathrm{s}$

$\mathrm{V}_{2}=3.87 \mathrm{~km} / \mathrm{s}$

Minimum depth to basalt bedrock $=20.85 \mathrm{~m}$

TABLE A111. SEISMIC LINE 20, SHOT 10

\begin{tabular}{lll}
\hline Geophone & Distance from Source $(\mathrm{ft})$ & Arrival Time $(\mathrm{ms})$ \\
\hline 1 & 120 & n.a. \\
2 & 110 & 48.2 \\
3 & 100 & 46.2 \\
4 & 90 & n.a. \\
5 & 80 & 45.0 \\
6 & 70 & 41.8 \\
7 & 60 & 39.0 \\
8 & 50 & n.a. \\
9 & 40 & n.a. \\
10 & 30 & 17.8 \\
11 & 20 & 9.0 \\
12 & 10 & n.a. \\
\hline n.a. =not available. &
\end{tabular}

CALCULATIONS FOR SHOT $($ GRADE $=B$ )

Slope $=0.1693$

$\mathrm{V}_{1}=1.80 \mathrm{~km} / \mathrm{s}$

$\mathrm{V}_{2}=3.87 \mathrm{~km} / \mathrm{s}$

Minimum depth to basalt bedrock $=40.22 \mathrm{~m}$ 
TABLE A112. SEISMIC LINE 21, SHOT 1

\begin{tabular}{lll}
\hline Geophone & Distance from Source $(\mathrm{ft})$ & Arrival Time $(\mathrm{ms})$ \\
\hline 1 & 10 & 6.6 \\
2 & 20 & 12.2 \\
3 & 30 & 16.6 \\
4 & 40 & 24.2 \\
5 & 50 & 28.6 \\
6 & 60 & 33.8 \\
7 & 70 & 40.6 \\
8 & 80 & n.a. \\
9 & 90 & n.a. \\
10 & 100 & n.a. \\
11 & 110 & 53.0 \\
12 & 120 & n.a. \\
\hline
\end{tabular}

n.a. $=$ not available.

\section{CALCULATIONS FOR SHOT (GRADE $=\mathrm{B})$}

Slope $=0.4960$

$\mathrm{V}_{1}=0.61 \mathrm{~km} / \mathrm{s}$

$\mathrm{V}_{2}=3.87 \mathrm{~km} / \mathrm{s}$

Minimum depth to basalt bedrock $=13.80 \mathrm{~m}$

TABLE A 1 13. SEISMIC LINE 21, SHOT 2

\begin{tabular}{lll}
\hline Geophone & Distance from Source $(\mathrm{ft})$ & Arrival Time $(\mathrm{ms})$ \\
\hline 1 & 120 & 109.4 \\
2 & 110 & 98.2 \\
3 & 100 & 90.2 \\
4 & 90 & 79.8 \\
5 & 80 & 71.8 \\
6 & 70 & 62.6 \\
7 & 60 & 52.6 \\
8 & 50 & n.a. \\
9 & 40 & n.a. \\
10 & 30 & n.a. \\
11 & 20 & 9.0 \\
12 & 10 & n.a. \\
\hline
\end{tabular}

\section{CALCULATIONS FOR SHOT $($ GRADE $=\mathbf{A})$}

Slope $=0.9054$

$\mathrm{V}_{1}=0.34 \mathrm{~km} / \mathrm{s}$

$\mathrm{V}_{2}=3.87 \mathrm{~km} / \mathrm{s}$

Minimum depth to basalt bedrock $=16.89 \mathrm{~m}$ 
TABLE A114. SEISMIC LINE 21. SHOT 3

\begin{tabular}{lll}
\hline Geophone & Distance from Source $(\mathrm{ft})$ & Arrival Time $(\mathrm{ms})$ \\
\hline 1 & 10 & 6.2 \\
2 & 20 & 15.4 \\
3 & 30 & 25.0 \\
4 & 40 & 31.8 \\
5 & 50 & 38.2 \\
6 & 60 & 44.2 \\
7 & 70 & 53.4 \\
8 & 80 & n.a. \\
9 & 90 & n.a. \\
10 & 100 & n.a. \\
11 & 110 & n.a. \\
12 & 120 & n.a. \\
\hline
\end{tabular}

n.a. $=$ not available.

CALCULATIONS FOR SHOT $($ GRADE $=$ A)

Slope $=0.7607$

$\mathrm{V}_{1}=0.40 \mathrm{~km} / \mathrm{s}$

$\mathrm{V}_{2}=3.87 \mathrm{~km} / \mathrm{s}$

Minimum depth to basalt bedrock $=9.65 \mathrm{~m}$

TABLE A115. SEISMIC LINE 21, SHOT 4

\begin{tabular}{lll}
\hline Geophone & Distance from Source (ft) & Arrival Time (ms) \\
\hline 1 & 120 & 108.2 \\
2 & 110 & 99.0 \\
3 & 100 & 85.0 \\
4 & 90 & 79.4 \\
5 & 80 & 70.2 \\
6 & 70 & 61.0 \\
7 & 60 & 51.8 \\
8 & 50 & n.a. \\
9 & 40 & n.a. \\
10 & 30 & 24.6 \\
11 & 20 & 15.8 \\
12 & 10 & n.a. \\
\hline
\end{tabular}

n.a. $=$ not available.

\section{CALCULATIONS FOR SHOT $($ GRADE $=$ A)}

Slope $=0.9003$

$\mathrm{V}_{1}=0.34 \mathrm{~km} / \mathrm{s}$

$\mathrm{V}_{2}=3.87 \mathrm{~km} / \mathrm{s}$

Minimum depth to basalt bedrock $=16.78 \mathrm{~m}$ 
TABLE A116. SEISMIC LINE 21, SHOT 5

\begin{tabular}{lll}
\hline Geophone & Distance from Source $(\mathrm{ft})$ & Arrival Time $(\mathrm{ms})$ \\
\hline 1 & 10 & 7.0 \\
2 & 20 & 14.6 \\
3 & 30 & 25.0 \\
4 & 40 & 33.8 \\
5 & 50 & 43.0 \\
6 & 60 & 51.8 \\
7 & 70 & 61.0 \\
8 & 80 & n.a. \\
9 & 90 & n.a. \\
10 & 100 & 87.8 \\
11 & 110 & 98.6 \\
12 & 120 & n.a. \\
\hline n.a.=not available. &
\end{tabular}

CALCULATIONS FOR SHOT $($ GRADE $=A)$

Slope $=0.9024$

$\mathrm{V}_{1}=0.34 \mathrm{~km} / \mathrm{s}$

$\mathrm{V}_{2}=3.87 \mathrm{~km} / \mathrm{s}$

Minimum depth to basalt bedrock $=15.25 \mathrm{~m}$

TABLE A117. SEISMIC LINE 21, SHOT 6

\begin{tabular}{lll}
\hline Geophone & Distance from Source $(\mathrm{ft})$ & Arrival Time $(\mathrm{ms})$ \\
\hline 1 & 120 & 109.4 \\
2 & 110 & 99.0 \\
3 & 100 & 89.8 \\
4 & 90 & 81.0 \\
5 & 80 & 71.8 \\
6 & 70 & 62.2 \\
7 & 60 & 53.8 \\
8 & 50 & n.a. \\
9 & 40 & n.a. \\
10 & 30 & 22.2 \\
11 & 20 & 16.2 \\
12 & 10 & n.a. \\
\hline
\end{tabular}

n.a. $=$ not available.

\section{CALCULATIONS FOR SHOT $($ GRADE $=A)$}

Slope $=0.9229$

$\mathrm{V}_{1}=0.33 \mathrm{~km} / \mathrm{s}$

$\mathrm{V}_{2}=3.87 \mathrm{~km} / \mathrm{s}$

Minimum depth to basalt bedrock $=16.57 \mathrm{~m}$ 
TABLE A118. SEISMIC LINE 21, SHOT 7

\begin{tabular}{lll}
\hline Geophone & Distance from Source $(\mathrm{ft})$ & Arrival Time $(\mathrm{ms})$ \\
\hline 1 & 10 & 7.4 \\
2 & 20 & 17.0 \\
3 & 30 & 25.0 \\
4 & 40 & 35.0 \\
5 & 50 & 44.6 \\
6 & 60 & 54.6 \\
7 & 70 & 62.6 \\
8 & 80 & n.a. \\
9 & 90 & n.a. \\
10 & 100 & 90.2 \\
11 & 110 & 100.6 \\
12 & 120 & n.a. \\
\hline n.a. $=$ not available. &
\end{tabular}

CALCULATIONS FOR SHOT (GRADE $=A)$

Slope $=0.9202$

$\mathrm{V}_{1}=0.33 \mathrm{~km} / \mathrm{s}$

$\mathrm{V}_{2}=3.87 \mathrm{~km} / \mathrm{s}$

Minimum depth to basalt bedrock $=15.29 \mathrm{~m}$

TABLE A1 19. SEISMIC LINE 21, SHOT 8

\begin{tabular}{lll}
\hline Geophone & Distance from Source $(\mathrm{ft})$ & Arrival Time $(\mathrm{ms})$ \\
\hline 1 & 120 & 104.6 \\
2 & 110 & 96.6 \\
3 & 100 & 86.6 \\
4 & 90 & 76.6 \\
5 & 80 & 67.8 \\
6 & 70 & 57.8 \\
7 & 60 & 49.4 \\
8 & 50 & n.a. \\
9 & 40 & n.a. \\
10 & 30 & 21.4 \\
11 & 20 & 13.0 \\
12 & 10 & n.a. \\
\hline n.a. $=$ not available. &
\end{tabular}

CALCULATIONS FOR SHOT $($ GRADE $=\mathrm{A})$

Slope $=0.8963$

$\mathrm{V}_{1}=0.34 \mathrm{~km} / \mathrm{s}$

$\mathrm{V}_{2}=3.87 \mathrm{~km} / \mathrm{s}$

Minimum depth to basalt bedrock $=16.24 \mathrm{~m}$ 
TABLE A120. SEISMIC LINE 21, SHOT 9

\begin{tabular}{lll}
\hline Geophone & Distance from Source $(\mathrm{ft})$ & Arrival Time $(\mathrm{ms})$ \\
\hline 1 & 0 & 3.4 \\
2 & 10 & 9.4 \\
3 & 20 & 19.4 \\
4 & 30 & 29.4 \\
5 & 40 & 35.8 \\
6 & 50 & 44.6 \\
7 & 60 & 53.8 \\
8 & 70 & n.a. \\
9 & 80 & n.a. \\
10 & 90 & 80.2 \\
11 & 100 & 84.2 \\
12 & 110 & n.a. \\
\hline n.a. $=$ not available. &
\end{tabular}

CALCULATIONS FOR SHOT $($ GRADE $=$ A)

Slope $=0.8567$

$\mathrm{V}_{1}=0.36 \mathrm{~km} / \mathrm{s}$

$\mathrm{V}_{2}=3.87 \mathrm{~km} / \mathrm{s}$

Minimum depth to basalt bedrock $=13.64 \mathrm{~m}$

TABLE A121. SEISMIC LINE 21, SHOT 10

\begin{tabular}{lll}
\hline Geophone & Distance from Source $(\mathrm{ft})$ & Arrival Time $(\mathrm{ms})$ \\
\hline 1 & 120 & 101.8 \\
2 & 110 & 93.4 \\
3 & 100 & 85.0 \\
4 & 90 & 74.6 \\
5 & 80 & 67.8 \\
6 & 70 & 59.0 \\
7 & 60 & 49.4 \\
8 & 50 & n.a. \\
9 & 40 & n.a. \\
10 & 30 & 24.2 \\
11 & 20 & 15.0 \\
12 & 10 & n.a. \\
\hline
\end{tabular}

n.a. $=$ not available.

CALCULATIONS FOR SHOT $(G R A D E=A)$

Slope $=0.8568$

$\mathrm{V}_{\mathrm{l}}=0.36 \mathrm{~km} / \mathrm{s}$

$\mathrm{V}_{2}=3.87 \mathrm{~km} / \mathrm{s}$

Minimum depth to basalt bedrock $=16.50 \mathrm{~m}$ 
TABLE A 122. SEISMIC LINE 22, SHOT 1

\begin{tabular}{lll}
\hline Geophone & Distance from Source $(\mathrm{ft})$ & Arrival Time $(\mathrm{ms})$ \\
\hline 1 & 10 & 7.8 \\
2 & 20 & 17.8 \\
3 & 30 & 27.4 \\
4 & 40 & 34.6 \\
5 & 50 & 43.4 \\
6 & 60 & 55.0 \\
7 & 70 & 67.0 \\
8 & 80 & n.a. \\
9 & 90 & n.a. \\
10 & 100 & 90.6 \\
11 & 110 & 98.6 \\
12 & 120 & n.a. \\
\hline n.a.=not available. &
\end{tabular}

\section{CALCULATIONS FOR SHOT $($ GRADE $=\mathrm{A})$}

Slope $=0.9130$

$\mathrm{V}_{\mathrm{I}}=0.33 \mathrm{~km} / \mathrm{s}$

$\mathrm{V}_{2}=3.87 \mathrm{~km} / \mathrm{s}$

Minimum depth to basalt bedrock $=15.07 \mathrm{~m}$

TABLE A123. SEISMIC LINE 22, SHOT 2

\begin{tabular}{lll}
\hline Geophone & Distance from Source $(\mathrm{ft})$ & Arrival Time $(\mathrm{ms})$ \\
\hline 1 & 120 & n.a. \\
2 & 110 & n.a. \\
3 & 100 & 68.6 \\
4 & 90 & 67.0 \\
5 & 80 & 65.4 \\
6 & 70 & 61.8 \\
7 & 60 & 52.2 \\
8 & 50 & n.a. \\
9 & 40 & n.a. \\
10 & 30 & 23.8 \\
11 & 20 & 16.2 \\
12 & 10 & n.a. \\
\hline
\end{tabular}

n.a. $=$ not available.

\section{CALCULATIONS FOR SHOT (GRADE $=\mathrm{A})$}

Slope $=0.1600$

$\mathrm{V}_{1}=1.91 \mathrm{~km} / \mathrm{s}$

$\mathrm{V}_{2}=3.87 \mathrm{~km} / \mathrm{s}$

Minimum depth to basalt bedrock $=66.47 \mathrm{~m}$ 
TABLE A124. SEISMIC LINE 22, SHOT 3

\begin{tabular}{lll}
\hline Geophone & Distance from Source $(\mathrm{ft})$ & Arrival Time $(\mathrm{ms})$ \\
\hline 1 & 10 & 7.0 \\
2 & 20 & 15.8 \\
3 & 30 & 24.6 \\
4 & 40 & 32.6 \\
5 & 50 & 43.8 \\
6 & 60 & 51.0 \\
7 & 70 & 59.8 \\
8 & 80 & n.a. \\
9 & 90 & n.a. \\
10 & 100 & 88.2 \\
11 & 110 & 97.4 \\
12 & 120 & n.a. \\
\hline n.a.=not available. &
\end{tabular}

CALCULATIONS FOR SHOT $($ GRADE $=A$ )

Slope $=0.8940$

$\mathrm{V}_{1}=0.34 \mathrm{~km} / \mathrm{s}$

$\mathrm{V}_{2}=3.87 \mathrm{~km} / \mathrm{s}$

Minimum depth to basalt bedrock $=15.19 \mathrm{~m}$

TABLE A125. SEISMIC LINE 22, SHOT 4

\begin{tabular}{lll}
\hline Geophone & Distance from Source $(\mathrm{ft})$ & Arrival Time $(\mathrm{ms})$ \\
\hline 1 & 120 & 107.4 \\
2 & 110 & 97.4 \\
3 & 100 & 89.0 \\
4 & 90 & 81.8 \\
5 & 80 & 71.4 \\
6 & 70 & 58.6 \\
7 & 60 & 53.0 \\
8 & 50 & n.a. \\
9 & 40 & n.a. \\
10 & 30 & 26.2 \\
11 & 20 & 17.4 \\
12 & 10 & n.a. \\
\hline
\end{tabular}

n.a. $=$ not available.

CALCULATIONS FOR SHOT $($ GRADE $=A$ )

Slope $=0.8958$

$\mathrm{V}_{1}=0.34 \mathrm{~km} / \mathrm{s}$

$V_{2}=3.87 \mathrm{~km} / \mathrm{s}$

Minimum depth to basalt bedrock $=16.73 \mathrm{~m}$ 
TABLE A126. SEISMIC LINE 27, SHOT 1

\begin{tabular}{lll}
\hline Geophone & Distance from Source $(\mathrm{ft})$ & Arrival Time $(\mathrm{ms})$ \\
\hline 1 & 10 & 7.0 \\
2 & 20 & 14.6 \\
3 & 30 & 18.6 \\
4 & 40 & 20.6 \\
5 & 50 & 21.8 \\
6 & 60 & 25.8 \\
7 & 70 & 28.2 \\
8 & 80 & n.a. \\
9 & 90 & 31.4 \\
10 & 100 & 32.6 \\
11 & 110 & 36.2 \\
12 & 120 & n.a. \\
\hline
\end{tabular}

n.a. $=$ not available.

\section{CALCULATIONS FOR SHOT (GRADE $=\mathrm{B}$ )}

Slope $=0.2152$

$\mathrm{V}_{\mathrm{I}}=1.42 \mathrm{~km} / \mathrm{s}$

$\mathrm{V}_{2}=3.87 \mathrm{~km} / \mathrm{s}$

Minimum depth to basalt bedrock $=20.96 \mathrm{~m}$

TABLE A127. SEISMIC LINE 27, SHOT 2

\begin{tabular}{lll}
\hline Geophone & Distance from Source $(\mathrm{ft})$ & Arrival Time $(\mathrm{ms})$ \\
\hline 1 & 120 & 29.8 \\
2 & 110 & 29.4 \\
3 & 100 & 27.0 \\
4 & 90 & 24.6 \\
5 & 80 & 22.6 \\
6 & 70 & 21.8 \\
7 & 60 & 19.4 \\
8 & 50 & n.a. \\
9 & 40 & 15.8 \\
10 & 30 & 15.4 \\
11 & 20 & 15.0 \\
12 & 10 & 9.4 \\
\hline n.a. $=$ not available. &
\end{tabular}

CALCULATIONS FOR SHOT $($ GRADE=B)

Slope $=0.1627$

$\mathrm{V}_{\mathrm{I}}=1.87 \mathrm{~km} / \mathrm{s}$

$\mathrm{V}_{2}=3.87 \mathrm{~km} / \mathrm{s}$

Minimum depth to basalt bedrock $=21.79 \mathrm{~m}$ 
TABLE A128. SEISMIC LINE 27, SHOT 3

\begin{tabular}{lll}
\hline Geophone & Distance from Source $(\mathrm{ft})$ & Arrival Time $(\mathrm{ms})$ \\
\hline 1 & 10 & 7.0 \\
2 & 20 & 14.2 \\
3 & 30 & 17.8 \\
4 & 40 & 19.8 \\
5 & 50 & 20.2 \\
6 & 60 & 21.0 \\
7 & 70 & 23.8 \\
8 & 80 & n.a. \\
9 & 90 & n.a. \\
10 & 100 & 25.4 \\
11 & 110 & 29.0 \\
12 & 120 & n.a. \\
\hline
\end{tabular}

n.a.=not available.

\section{CALCULATIONS FOR SHOT $(G R A D E=D)$}

Slope $=0.1400$

$\mathrm{V}_{1}=2.18 \mathrm{~km} / \mathrm{s}$

$\mathrm{V}_{2}=3.87 \mathrm{~km} / \mathrm{s}$

Minimum depth to basalt bedrock $=26.78 \mathrm{~m}$

TABLE A129. SEISMIC LINE 27, SHOT 4

\begin{tabular}{lll}
\hline Geophone & Distance from Source (ft) & Arrival Time (ms) \\
\hline 1 & 120 & 32.6 \\
2 & 110 & 30.6 \\
3 & 100 & 32.2 \\
4 & 90 & 29.8 \\
5 & 80 & 28.2 \\
6 & 70 & 26.2 \\
7 & 60 & n.a. \\
8 & 50 & n.a. \\
9 & 40 & n.a. \\
10 & 30 & 18.6 \\
11 & 20 & 17.4 \\
12 & 10 & 7.8 \\
\hline n.a.=not available. &
\end{tabular}

\section{CALCULATIONS FOR SHOT (GRADE $=\mathrm{A})$}

Slope $=0.1821$

$\mathrm{V}_{1}=1.67 \mathrm{~km} / \mathrm{s}$

$\mathrm{V}_{2}=3.87 \mathrm{~km} / \mathrm{s}$

Minimum depth to basalt bedrock $=21.09 \mathrm{~m}$ 
TABLE A130. SEISMIC LINE 27, SHOT 5

\begin{tabular}{lll}
\hline Geophone & Distance from Source $(\mathrm{ft})$ & Arrival Time $(\mathrm{ms})$ \\
\hline 1 & 10 & 8.6 \\
2 & 20 & 14.6 \\
3 & 30 & 20.6 \\
4 & 40 & 24.2 \\
5 & 50 & 25.4 \\
6 & 60 & 28.2 \\
7 & 70 & 27.8 \\
8 & 80 & n.a. \\
9 & 90 & n.a. \\
10 & 100 & 32.6 \\
11 & 110 & 35.8 \\
12 & 120 & n.a. \\
\hline
\end{tabular}

n.a. $=$ not available.

\section{CALCULATIONS FOR SHOT (GRADE $=\mathrm{B})$}

Slope $=0.1683$

$V_{1}=1.81 \mathrm{~km} / \mathrm{s}$

$\mathrm{V}_{2}=3.87 \mathrm{~km} / \mathrm{s}$

Minimum depth to basalt bedrock $=27.81 \mathrm{~m}$

TABLE A131. SEISMIC LINE 27, SHOT 6

\begin{tabular}{lll}
\hline Geophone & Distance from Source $(\mathrm{ft})$ & Arrival Time $(\mathrm{ms})$ \\
\hline 1 & 120 & 39.8 \\
2 & 110 & 36.6 \\
3 & 100 & 34.2 \\
4 & 90 & 32.2 \\
5 & 80 & 29.8 \\
6 & 70 & 30.2 \\
7 & 60 & 30.2 \\
8 & 50 & n.a. \\
9 & 40 & n.a. \\
10 & 30 & 26.6 \\
11 & 20 & 16.6 \\
12 & 10 & 8.2 \\
\hline n.a. $=$ not available. &
\end{tabular}

\section{CALCULATIONS FOR SHOT $($ GRADE $=\mathrm{B})$}

Slope $=0.1563$

$\mathrm{V}_{1}=1.95 \mathrm{~km} / \mathrm{s}$

$\mathrm{V}_{2}=3.87 \mathrm{~km} / \mathrm{s}$

Minimum depth to basalt bedrock $=34.27 \mathrm{~m}$ 
TABLE A132. SEISMIC LINE 27, SHOT 7

\begin{tabular}{lll}
\hline Geophone & Distance from Source $(\mathrm{ft})$ & Arrival Time $(\mathrm{ms})$ \\
\hline 1 & 10 & 8.2 \\
2 & 20 & 18.2 \\
3 & 30 & 22.2 \\
4 & 40 & 25.4 \\
5 & 50 & 26.2 \\
6 & 60 & 31.0 \\
7 & 70 & 35.0 \\
8 & 80 & n.a. \\
9 & 90 & n.a. \\
10 & 100 & n.a. \\
11 & 110 & 47.0 \\
12 & 120 & n.a. \\
\hline n.a. $=$ not available. &
\end{tabular}

CALCULATIONS FOR SHOT (GRADE $=$ A)

Slope $=0.3179$

$\mathrm{V}_{\mathrm{I}}=0.96 \mathrm{~km} / \mathrm{s}$

$\mathrm{V}_{2}=3.87 \mathrm{~km} / \mathrm{s}$

Minimum depth to basalt bedrock $=18.97 \mathrm{~m}$

TABLE A133. SEISMIC LINE 27, SHOT 8

\begin{tabular}{lll}
\hline Geophone & Distance from Source $(\mathrm{ft})$ & Arrival Time $(\mathrm{ms})$ \\
\hline 1 & 120 & 54.6 \\
2 & 110 & 54.2 \\
3 & 100 & 53.8 \\
4 & 90 & 52.6 \\
5 & 80 & 50.6 \\
6 & 70 & 49.4 \\
7 & 60 & 45.4 \\
8 & 50 & n.a. \\
9 & 40 & n.a. \\
10 & 30 & 26.2 \\
11 & 20 & 16.6 \\
12 & 10 & n.a. \\
\hline n.a.=not available. &
\end{tabular}

n.a. $=$ not available.

CALCULATIONS FOR SHOT $(G R A D E=B)$

Slope $=0.1443$

$\mathrm{V}_{\mathrm{1}}=2.11 \mathrm{~km} / \mathrm{s}$

$\mathrm{V}_{2}=3.87 \mathrm{~km} / \mathrm{s}$

Minimum depth to basalt bedrock $=56.92 \mathrm{~m}$ 
TABLE A134. SEISMIC LINE 27, SHOT 9

\begin{tabular}{lll}
\hline Geophone & Distance from Source $(\mathrm{ft})$ & Arrival Time $(\mathrm{ms})$ \\
\hline 1 & 10 & 8.2 \\
2 & 20 & 17.0 \\
3 & 30 & 25.8 \\
4 & 40 & 37.4 \\
5 & 50 & 42.2 \\
6 & 60 & 47.0 \\
7 & 70 & 49.4 \\
8 & 80 & n.a. \\
9 & 90 & n.a. \\
10 & 100 & 53.0 \\
11 & 110 & 56.6 \\
12 & 120 & n.a. \\
\hline na $=$ not available & &
\end{tabular}

CALCULATIONS FOR SHOT $($ GRADE $=\mathrm{C})$

Slope $=0.2426$

$\mathrm{V}_{\mathrm{l}}=1.26 \mathrm{~km} / \mathrm{s}$

$\mathrm{V}_{2}=3.87 \mathrm{~km} / \mathrm{s}$

Minimum depth to basalt bedrock $=31.85 \mathrm{~m}$

TABLE A 135. SEISMIC LINE 27, SHOT 10

\begin{tabular}{lll}
\hline Geophone & Distance from Source $(\mathrm{ft})$ & Arrival Time $(\mathrm{ms})$ \\
\hline 1 & 120 & 55.8 \\
2 & 110 & 55.4 \\
3 & 100 & 56.6 \\
4 & 90 & 51.4 \\
5 & 80 & 47.4 \\
6 & 70 & 43.4 \\
7 & 60 & 41.8 \\
8 & 50 & n.a. \\
9 & 40 & n.a. \\
10 & 30 & 27.0 \\
11 & 20 & 17.0 \\
12 & 10 & n.a. \\
\hline n.a. $=$ not available. &
\end{tabular}

CALCULATIONS FOR SHOT (GRADE $=$ B)

Slope $=0.3760$

$\mathrm{V}_{1}=0.81 \mathrm{~km} / \mathrm{s}$

$\mathrm{V}_{2}=3.87 \mathrm{~km} / \mathrm{s}$

Minimum depth to basalt bedrock $=20.20 \mathrm{~m}$ 
TABLE A136. SEISMIC LINE 27, SHOT 11

\begin{tabular}{lll}
\hline Geophone & Distance from Source $(\mathrm{ft})$ & Arrival Time $(\mathrm{ms})$ \\
\hline 1 & 10 & 8.6 \\
2 & 20 & 19.8 \\
3 & 30 & 29.0 \\
4 & 40 & 33.4 \\
5 & 50 & 38.2 \\
6 & 60 & 40.6 \\
7 & 70 & 43.8 \\
8 & 80 & n.a. \\
9 & 90 & n.a. \\
10 & 100 & n.a. \\
11 & 110 & n.a. \\
12 & 120 & n.a. \\
\hline n.a. $=$ not available. &
\end{tabular}

CALCULATIONS FOR SHOT (GRADE $=A$ )

Slope $=0.3680$

$V_{1}=0.83 \mathrm{~km} / \mathrm{s}$

$\mathrm{V}_{2}=3.87 \mathrm{~km} / \mathrm{s}$

Minimum depth to basalt bedrock $=16.24 \mathrm{~m}$

TABLE A137. SEISMIC LINE 27, SHOT 12

\begin{tabular}{lll}
\hline Geophone & Distance from Source $(\mathrm{ft})$ & Arrival Time $(\mathrm{ms})$ \\
\hline 1 & 120 & 58.6 \\
2 & 110 & n.a. \\
3 & 100 & n.a. \\
4 & 90 & 44.6 \\
5 & 80 & 43.8 \\
6 & 70 & 42.6 \\
7 & 60 & n.a. \\
8 & 50 & n.a. \\
9 & 40 & n.a. \\
10 & 30 & 29.4 \\
11 & 20 & 19.8 \\
12 & 10 & n.a. \\
\hline
\end{tabular}

n.a.=not available.

CALCULATIONS FOR SHOT $($ GRADE $=C)$

Slope $=0.3343$

$V_{1}=0.91 \mathrm{~km} / \mathrm{s}$

$V_{2}=3.87 \mathrm{~km} / \mathrm{s}$

Minimum depth to basalt bedrock $=23.06 \mathrm{~m}$ 
TABLE AI38. SEISMIC LINE 27, SHOT 13

\begin{tabular}{lll}
\hline Geophone & Distance from Source $(\mathrm{ft})$ & Arrival Time $(\mathrm{ms})$ \\
\hline $\mathrm{l}$ & 10 & 8.6 \\
2 & 20 & 18.2 \\
3 & 30 & 29.0 \\
4 & 40 & 36.2 \\
5 & 50 & 36.6 \\
6 & 60 & 40.6 \\
7 & 70 & 44.6 \\
8 & 80 & n.a. \\
9 & 90 & n.a. \\
10 & 100 & 46.2 \\
11 & 110 & 49.8 \\
12 & 120 & n.a. \\
\hline
\end{tabular}

n.a. $=$ not available.

CALCULATIONS FOR SHOT $($ GRADE $=C)$

Slope $=0.1882$

$\mathrm{V}_{\mathrm{I}}=1.62 \mathrm{~km} / \mathrm{s}$

$\mathrm{V}_{2}=3.87 \mathrm{~km} / \mathrm{s}$

Minimum depth to basalt bedrock $=36.69 \mathrm{~m}$

TABLE A139. SEISMIC LINE 27, SHOT 14

\begin{tabular}{lll}
\hline Geophone & Distance from Source $(\mathrm{ft})$ & Arrival Time $(\mathrm{ms})$ \\
\hline 1 & 120 & 57.8 \\
2 & 110 & 50.6 \\
3 & 100 & 49.4 \\
4 & 90 & 49.0 \\
5 & 80 & 44.2 \\
6 & 70 & 40.6 \\
7 & 60 & 38.6 \\
8 & 50 & n.a. \\
9 & 40 & n.a. \\
10 & 30 & 25.8 \\
11 & 20 & 17.4 \\
12 & 10 & n.a. \\
\hline
\end{tabular}

n.a. $=$ not available.

\section{CALCULATIONS FOR SHOT $(G R A D E=C)$}

Slope $=0.2957$

$\mathrm{V}_{\mathrm{l}}=1.03 \mathrm{~km} / \mathrm{s}$

$\mathrm{V}_{2}=3.87 \mathrm{~km} / \mathrm{s}$

Minimum depth to basalt bedrock $=25.86 \mathrm{~m}$ 
TABLE AI 40. SEISMIC LINE 27, SHOT 15

\begin{tabular}{lll}
\hline Geophone & Distance from Source $(\mathrm{ft})$ & Arrival Time $(\mathrm{ms})$ \\
\hline 1 & 10 & 9.0 \\
2 & 20 & 17.0 \\
3 & 30 & 27.4 \\
4 & 40 & 31.8 \\
5 & 50 & 35.0 \\
6 & 60 & 36.6 \\
7 & 70 & 39.8 \\
8 & 80 & n.a. \\
9 & 90 & n.a. \\
10 & 100 & n.a. \\
11 & 110 & n.a. \\
12 & 120 & n.a. \\
\hline
\end{tabular}

n.a. $=$ not available.

CALCULATIONS FOR SHOT $($ GRADE $=\mathrm{A})$

Slope $=0.2960$

$\mathrm{V}_{1}=1.03 \mathrm{~km} / \mathrm{s}$

$\mathrm{V}_{2}=3.87 \mathrm{~km} / \mathrm{s}$

Minimum depth to basalt bedrock $=18.32 \mathrm{~m}$

TABLE A141. SEISMIC LINE 27, SHOT 16

\begin{tabular}{lll}
\hline Geophone & Distance from Source $(\mathrm{ft})$ & Arrival Time $(\mathrm{ms})$ \\
\hline 1 & 120 & 48.2 \\
2 & 110 & 46.2 \\
3 & 100 & 43.8 \\
4 & 90 & 39.8 \\
5 & 80 & 39.0 \\
6 & 70 & 36.6 \\
7 & 60 & 35.0 \\
8 & 50 & n.a. \\
9 & 40 & n.a. \\
10 & 30 & 27.8 \\
11 & 20 & 20.6 \\
12 & 10 & 13.4 \\
\hline n.a. $=$ not available. &
\end{tabular}

CALCULATIONS FOR SHOT $(G R A D E=B)$

Slope $=0.1680$

$\mathrm{V}_{1}=1.81 \mathrm{~km} / \mathrm{s}$

$\mathrm{V}_{2}=3.87 \mathrm{~km} / \mathrm{s}$

Minimum depth to basalt bedrock $=33.60 \mathrm{~m}$ 
TABLE A142. SEISMIC LINE 27. SHOT 17

\begin{tabular}{lll}
\hline Geophone & Distance from Source $(\mathrm{ft})$ & Arrival Time $(\mathrm{ms})$ \\
\hline 1 & 10 & 2.2 \\
2 & 20 & 11.0 \\
3 & 30 & 17.4 \\
4 & 40 & 21.0 \\
5 & 50 & 25.0 \\
6 & 60 & 32.2 \\
7 & 70 & 32.6 \\
8 & 80 & n.a. \\
9 & 90 & n.a. \\
10 & 100 & 39.4 \\
11 & 110 & 42.6 \\
12 & 120 & n.a. \\
\hline
\end{tabular}

n.a. $=$ not available.

CALCULATIONS FOR SHOT (GRADE=B)

Slope $=0.3051$

$\mathrm{V}_{1}=1.00 \mathrm{~km} / \mathrm{s}$

$\mathrm{V}_{2}=3.87 \mathrm{~km} / \mathrm{s}$

Minimum depth to basalt bedrock $=17.55 \mathrm{~m}$

TABLE A143. SEISMIC LINE 27, SHOT 18

\begin{tabular}{lll}
\hline Geophone & Distance from Source $(\mathrm{ft})$ & Arrival Time $(\mathrm{ms})$ \\
\hline 1 & 120 & n.a. \\
2 & 110 & n.a. \\
3 & 100 & n.a. \\
4 & 90 & 49.4 \\
5 & 80 & n.a. \\
6 & 70 & 37.8 \\
7 & 60 & 33.8 \\
8 & 50 & n.a. \\
9 & 40 & n.a. \\
10 & 30 & 23.8 \\
11 & 20 & 19.4 \\
12 & 10 & n.a. \\
\hline
\end{tabular}

n.a. $=$ not available.

CALCULATIONS FOR SHOT $($ GRADE $=B$ )

Slope $=0.4082$

$\mathrm{V}_{1}=0.75 \mathrm{~km} / \mathrm{s}$

$\mathrm{V}_{2}=3.87 \mathrm{~km} / \mathrm{s}$

Minimum depth to basalt bedrock $=16.10 \mathrm{~m}$ 
TABLE A144. SEISMIC LINE 28, SHOT 1

\begin{tabular}{lll}
\hline Geophone & Distance from Source (ft) & Arrival Time $(\mathrm{ms})$ \\
\hline 1 & 10 & 9.4 \\
2 & 20 & 12.6 \\
3 & 30 & 16.6 \\
4 & 40 & 25.8 \\
5 & 50 & 28.2 \\
6 & 60 & 31.4 \\
7 & 70 & 33.0 \\
8 & 80 & n.a. \\
9 & 90 & n.a. \\
10 & 100 & 39.8 \\
11 & 110 & 41.8 \\
12 & 120 & n.a. \\
n.a.=not available. &
\end{tabular}

\section{CALCULATIONS FOR SHOT $($ GRADE $=\mathrm{A})$}

Slope $=0.2268$

$\mathrm{V}_{1}=1.34 \mathrm{~km} / \mathrm{s}$

$\mathrm{V}_{2}=3.87 \mathrm{~km} / \mathrm{s}$

Minimum depth to basalt bedrock $=23.75 \mathrm{~m}$

TABLE A145. SEISMIC LINE 28. SHOT 2

\begin{tabular}{lll}
\hline Geophone & Distance from Source $(\mathrm{ft})$ & Arrival Time $(\mathrm{ms})$ \\
\hline 1 & 120 & 49.4 \\
2 & 110 & 49.4 \\
3 & 100 & 52.2 \\
4 & 90 & 51.8 \\
5 & 80 & 51.0 \\
6 & 70 & 49.0 \\
7 & 60 & 48.2 \\
8 & 50 & n.a. \\
9 & 40 & n.a. \\
10 & 30 & 27.0 \\
11 & 20 & 18.6 \\
12 & 10 & n.a. \\
\hline
\end{tabular}

n.a. $=$ not available.

CALCULATIONS FOR SHOT (GRADE $=\mathrm{B})$

Slope $=0.1080$

$\mathrm{V}_{\mathrm{l}}=2.82 \mathrm{~km} / \mathrm{s}$

$\mathrm{V}_{2}=3.87 \mathrm{~km} / \mathrm{s}$

Minimum depth to basalt bedrock $=91.46 \mathrm{~m}$ 
TABLE A146. SEISMIC LINE 28, SHOT 3

\begin{tabular}{lll}
\hline Geophone & Distance from Source $(\mathrm{ft})$ & Arrival Time $(\mathrm{ms})$ \\
\hline 1 & 10 & 8.6 \\
2 & 20 & 18.2 \\
3 & 30 & 26.2 \\
4 & 40 & 35.8 \\
5 & 50 & 45.0 \\
6 & 60 & 53.8 \\
7 & 70 & 63.0 \\
8 & 80 & n.a. \\
9 & 90 & n.a. \\
10 & 100 & 89.8 \\
11 & 110 & n.a. \\
12 & 120 & n.a. \\
\hline n.a. $=$ not available. &
\end{tabular}

CALCULATIONS FOR SHOT $($ GRADE $=A)$

Slope $=0.9004$

$\mathrm{V}_{\mathrm{I}}=0.34 \mathrm{~km} / \mathrm{s}$

$\mathrm{V}_{2}=3.87 \mathrm{~km} / \mathrm{s}$

Minimum depth to basalt bedrock $=13.92 \mathrm{~m}$

TABLE A147. SEISMIC LINE 28, SHOT 4

\begin{tabular}{lll}
\hline Geophone & Distance from Source $(\mathrm{ft})$ & Arrival Time $(\mathrm{ms})$ \\
\hline 1 & 120 & 104.6 \\
2 & 110 & 99.0 \\
3 & 100 & 89.8 \\
4 & 90 & 80.2 \\
5 & 80 & 71.0 \\
6 & 70 & 61.4 \\
7 & 60 & 52.6 \\
8 & 50 & n.a. \\
9 & 40 & n.a. \\
10 & 30 & 25.8 \\
11 & 20 & 17.0 \\
12 & 10 & n.a. \\
\hline
\end{tabular}

n.a. $=$ not available.

CALCULATIONS FOR SHOT $(G R A D E=A)$

Slope $=0.8924$

$\mathrm{V}_{1}=0.34 \mathrm{~km} / \mathrm{s}$

$\mathrm{V}_{2}=3.87 \mathrm{~km} / \mathrm{s}$

Minimum depth to basalt bedrock $=16.32 \mathrm{~m}$ 
TABLE A148. SEISMIC LINE 28, SHOT 5

\begin{tabular}{lll}
\hline Geophone & Distance from Source $(\mathrm{ft})$ & Arrival Time $(\mathrm{ms})$ \\
\hline 1 & 10 & 9.4 \\
2 & 20 & 18.2 \\
3 & 30 & 27.4 \\
4 & 40 & 36.2 \\
5 & 50 & 45.0 \\
6 & 60 & 53.0 \\
7 & 70 & 62.6 \\
8 & 80 & n.a. \\
9 & 90 & n.a. \\
10 & 100 & 89.4 \\
11 & 110 & 98.2 \\
12 & 120 & n.a. \\
\hline
\end{tabular}

n.a. $=$ not available.

CALCULATIONS FOR SHOT (GRADE=A)

Slope $=0.8893$

$\mathrm{V}_{1}=0.34 \mathrm{~km} / \mathrm{s}$

$\mathrm{V}_{2}=3.87 \mathrm{~km} / \mathrm{s}$

Minimum depth to basalt bedrock $=15.41 \mathrm{~m}$

TABLE A149. SEISMIC LINE 28, SHOT 6

\begin{tabular}{lll}
\hline Geophone & Distance from Source $(\mathrm{ft})$ & Arrival Time $(\mathrm{ms})$ \\
\hline 1 & 120 & 106.6 \\
2 & 110 & 97.8 \\
3 & 100 & 87.8 \\
4 & 90 & 78.6 \\
5 & 80 & 70.2 \\
6 & 70 & 61.4 \\
7 & 60 & 47.8 \\
8 & 50 & n.a. \\
9 & 40 & n.a. \\
10 & 30 & 23.8 \\
11 & 20 & 15.0 \\
12 & 10 & n.a. \\
\hline
\end{tabular}

n.a.=not available.

CALCULATIONS FOR SHOT $($ GRADE $=\mathrm{A})$

Slope $=0.9030$

$\mathrm{V}_{1}=0.34 \mathrm{~km} / \mathrm{s}$

$\mathrm{V}_{2}=3.87 \mathrm{~km} / \mathrm{s}$

Minimum depth to basalt bedrock $=16.46 \mathrm{~m}$ 
TABLE A150. SEISMIC LINE 28. SHOT 7

\begin{tabular}{lll}
\hline Geophone & Distance from Source $(\mathrm{ft})$ & Arrival Time $(\mathrm{ms})$ \\
\hline 1 & 10 & 9.0 \\
2 & 20 & 18.2 \\
3 & 30 & 27.0 \\
4 & 40 & 36.6 \\
5 & 50 & 44.6 \\
6 & 60 & 54.6 \\
7 & 70 & 63.0 \\
8 & 80 & n.a. \\
9 & 90 & n.a. \\
10 & 100 & 90.6 \\
11 & 110 & 98.2 \\
12 & 120 & n.a. \\
\hline n.a.=not available & &
\end{tabular}

CALCULATIONS FOR SHOT (GRADE=A)

Slope $=0.8981$

$\mathrm{V}_{1}=0.34 \mathrm{~km} / \mathrm{s}$

$\mathrm{V}_{2}=3.87 \mathrm{~km} / \mathrm{s}$

Minimum depth to basalt bedrock $=15.26 \mathrm{~m}$

TABLE A151. SEISMIC LINE 28, SHOT 8

\begin{tabular}{lll}
\hline Geophone & Distance from Source $(\mathrm{ft})$ & Arrival Time $(\mathrm{ms})$ \\
\hline 1 & 120 & 107.0 \\
2 & 110 & 97.4 \\
3 & 100 & 89.0 \\
4 & 90 & 79.8 \\
5 & 80 & 75.0 \\
6 & 70 & 68.6 \\
7 & 60 & 63.4 \\
8 & 50 & n.a. \\
9 & 40 & n.a. \\
10 & 30 & n.a. \\
11 & 20 & n.a. \\
12 & 10 & n.a. \\
\hline n.a.=not available. &
\end{tabular}

CALCULATIONS FOR SHOT (GRADE $=$ A)

Slope $=0.8649$

$V_{1}=0.35 \mathrm{~km} / \mathrm{s}$

$\mathrm{V}_{2}=3.87 \mathrm{~km} / \mathrm{s}$

Minimum depth to basalt bedrock $=17.26 \mathrm{~m}$ 
TABLE A152. SEISMIC LINE 28, SHOT 9

\begin{tabular}{lll}
\hline Geophone & Distance from Source $(\mathrm{ft})$ & Arrival Time $(\mathrm{ms})$ \\
\hline 1 & 10 & 9.8 \\
2 & 20 & 17.4 \\
3 & 30 & 27.8 \\
4 & 40 & 35.0 \\
5 & 50 & 43.0 \\
6 & 60 & 48.2 \\
7 & 70 & 52.6 \\
8 & 80 & n.a. \\
9 & 90 & n.a. \\
10 & 100 & n.a. \\
11 & 110 & n.a. \\
12 & 120 & n.a. \\
\hline
\end{tabular}

n.a.=not available.

CALCULATIONS FOR SHOT (GRADE $=\mathrm{A})$

Slope $=0.7669$

$\mathrm{V}_{1}=0.40 \mathrm{~km} / \mathrm{s}$

$\mathrm{V}_{2}=3.87 \mathrm{~km} / \mathrm{s}$

Minimum depth to basalt bedrock $=9.41 \mathrm{~m}$

TABLE A153. SEISMIC LINE 28. SHOT 10

\begin{tabular}{lll}
\hline Geophone & Distance from Source $(\mathrm{ft})$ & Arrival Time $(\mathrm{ms})$ \\
\hline 1 & 120 & 108.2 \\
2 & 110 & 98.6 \\
3 & 100 & 90.2 \\
4 & 90 & 81.0 \\
5 & 80 & 72.2 \\
6 & 70 & 64.2 \\
7 & 60 & 55.0 \\
8 & 50 & n.a. \\
9 & 40 & n.a. \\
10 & 30 & 34.2 \\
11 & 20 & 19.8 \\
12 & 10 & n.a. \\
\hline n.a. $=$ not available. &
\end{tabular}

\section{CALCULATIONS FOR SHOT (GRADE $=\mathrm{A})$}

Slope $=0.8751$

$\mathrm{V}_{\mathrm{I}}=0.35 \mathrm{~km} / \mathrm{s}$

$\mathrm{V}_{2}=3.87 \mathrm{~km} / \mathrm{s}$

Minimum depth to basalt bedrock $=17.27 \mathrm{~m}$ 
TABLE A 154. SEISMIC LINE 28. SHOT 11

\begin{tabular}{lll}
\hline Geophone & Distance from Source $(\mathrm{ft})$ & Arrival Time $(\mathrm{ms})$ \\
\hline 1 & 10 & 8.6 \\
2 & 20 & 19.8 \\
3 & 30 & 36.6 \\
4 & 40 & 42.6 \\
5 & 50 & 45.8 \\
6 & 60 & 53.8 \\
7 & 70 & 57.0 \\
8 & 80 & n.a. \\
9 & 90 & n.a. \\
10 & 100 & 65.4 \\
11 & 110 & 71.8 \\
12 & 120 & n.a. \\
\hline n.a.=not available & &
\end{tabular}

CALCULATIONS FOR SHOT (GRADE $=\mathrm{A})$

Slope $=0.4164$

$\mathrm{V}_{1}=0.73 \mathrm{~km} / \mathrm{s}$

$\mathrm{V}_{2}=3.87 \mathrm{~km} / \mathrm{s}$

Minimum depth to basalt bedrock $=23.54 \mathrm{~m}$

TABLE A155. SEISMIC LINE 28, SHOT 12

\begin{tabular}{lll}
\hline Geophone & Distance from Source $(\mathrm{ft})$ & Arrival Time $(\mathrm{ms})$ \\
\hline 1 & 120 & 62.6 \\
2 & 110 & 59.8 \\
3 & 100 & 57.4 \\
4 & 90 & 52.6 \\
5 & 80 & n.a. \\
6 & 70 & 38.2 \\
7 & 60 & 35.4 \\
8 & 50 & n.a. \\
9 & 40 & n.a. \\
10 & 30 & 26.6 \\
11 & 20 & 19.0 \\
12 & 10 & 15.4 \\
\hline n.a. $=$ not available. &
\end{tabular}

\section{CALCULATIONS FOR SHOT $(\mathrm{GRADE}=\mathrm{B})$}

Slope $=0.4424$

$\mathrm{V}_{1}=0.69 \mathrm{~km} / \mathrm{s}$

$\mathrm{V}_{2}=3.87 \mathrm{~km} / \mathrm{s}$

Minimum depth to basalt bedrock $=18.61 \mathrm{~m}$ 
TABLE A156. SEISMIC LINE 28, SHOT 13

\begin{tabular}{lll}
\hline Geophone & Distance from Source $(\mathrm{ft})$ & Arrival Time $(\mathrm{ms})$ \\
\hline 1 & 10 & 6.2 \\
2 & 20 & 14.6 \\
3 & 30 & 24.2 \\
4 & 40 & 30.2 \\
5 & 50 & 33.4 \\
6 & 60 & 33.8 \\
7 & 70 & 37.8 \\
8 & 80 & n.a. \\
9 & 90 & n.a. \\
10 & 100 & n.a. \\
11 & 110 & 45.4 \\
12 & 120 & n.a. \\
\hline n.a.=not available. &
\end{tabular}

\section{CALCULATIONS FOR SHOT (GRADE=B)}

Slope $=0.2145$

$\mathrm{V}_{\mathrm{I}}=1.42 \mathrm{~km} / \mathrm{s}$

$\mathrm{V}_{2}=3.87 \mathrm{~km} / \mathrm{s}$

Minimum depth to basalt bedrock $=28.07 \mathrm{~m}$

TABLE A157. SEISMIC LINE 28, SHOT 14

\begin{tabular}{lll}
\hline Geophone & Distance from Source $(\mathrm{ft})$ & Arrival Time $(\mathrm{ms})$ \\
\hline 1 & 120 & 51.4 \\
2 & 110 & 49.8 \\
3 & 100 & 48.2 \\
4 & 90 & 45.4 \\
5 & 80 & 43.0 \\
6 & 70 & 41.4 \\
7 & 60 & 38.6 \\
8 & 50 & n.a. \\
9 & 40 & n.a. \\
10 & 30 & 25.8 \\
11 & 20 & 16.2 \\
12 & 10 & n.a. \\
\hline
\end{tabular}

n.a. $=$ not available.

CALCULATIONS FOR SHOT (GRADE $=\mathrm{A})$

Slope $=0.2157$

$\mathrm{V}_{1}=1.41 \mathrm{~km} / \mathrm{s}$

$\mathrm{V}_{2}=3.87 \mathrm{~km} / \mathrm{s}$

Minimum depth to basalt bedrock $=31.84 \mathrm{~m}$ 
TABLE A 158. SEISMIC LINE 28. SHOT 15

\begin{tabular}{lll}
\hline Geophone & Distance from Source $(\mathrm{ft})$ & Arrival Time $(\mathrm{ms})$ \\
\hline 1 & 10 & 7.8 \\
2 & 20 & 17.4 \\
3 & 30 & 27.4 \\
4 & 40 & 34.2 \\
5 & 50 & 37.8 \\
6 & 60 & 39.8 \\
7 & 70 & 42.2 \\
8 & 80 & n.a. \\
9 & 90 & n.a. \\
10 & 100 & 46.6 \\
11 & 110 & 48.2 \\
12 & 120 & n.a. \\
\hline n.a. $=$ not available. &
\end{tabular}

\section{CALCULATIONS FOR SHOT (GRADE $=\mathrm{B}$ )}

Slope $=0.1883$

$\mathrm{V}_{\mathrm{I}}=1.62 \mathrm{~km} / \mathrm{s}$

$V_{2}=3.87 \mathrm{~km} / \mathrm{s}$

Minimum depth to basalt bedrock $=35.24 \mathrm{~m}$

TABLE A159. SEISMIC LINE 28, SHOT 16

\begin{tabular}{lll}
\hline Geophone & Distance from Source $(\mathrm{ft})$ & Arrival Time $(\mathrm{ms})$ \\
\hline 1 & 120 & 64.2 \\
2 & 110 & 62.2 \\
3 & 100 & 57.8 \\
4 & 90 & 52.2 \\
5 & 80 & 50.2 \\
6 & 70 & 49.4 \\
7 & 60 & 42.6 \\
8 & 50 & n.a. \\
9 & 40 & n.a. \\
10 & 30 & 27.0 \\
11 & 20 & 17.8 \\
12 & 10 & n.a. \\
\hline n.a.=not available. &
\end{tabular}

\section{CALCULATIONS FOR SHOT (GRADE=B)}

Slope $=0.3500$

$\mathrm{V}_{\mathrm{I}}=0.87 \mathrm{~km} / \mathrm{s}$

$\mathrm{V}_{2}=3.87 \mathrm{~km} / \mathrm{s}$

Minimum depth to basalt bedrock $=24.47 \mathrm{~m}$ 
TABLE A160. SEISMIC LINE 28, SHOT 17

\begin{tabular}{lll}
\hline Geophone & Distance from Source $(\mathrm{ft})$ & Arrival Time $(\mathrm{ms})$ \\
\hline 1 & 10 & 7.8 \\
2 & 20 & 17.0 \\
3 & 30 & 26.6 \\
4 & 40 & 33.0 \\
5 & 50 & 36.2 \\
6 & 60 & 38.2 \\
7 & 70 & 38.6 \\
8 & 80 & n.a. \\
9 & 90 & n.a. \\
10 & 100 & 43.8 \\
11 & 110 & 49.0 \\
12 & 120 & n.a. \\
\hline
\end{tabular}

n.a.=not available.

CALCULATIONS FOR SHOT $(G R A D E=B)$

Slope $=0.1683$

$\mathrm{V}_{1}=1.81 \mathrm{~km} / \mathrm{s}$

$\mathrm{V}_{2}=3.87 \mathrm{~km} / \mathrm{s}$

Minimum depth to basalt bedrock $=36.82 \mathrm{~m}$

TABLE A161. SEISMIC LINE 28, SHOT 18

\begin{tabular}{lll}
\hline Geophone & Distance from Source (ft) & Arrival Time $(\mathrm{ms})$ \\
\hline 1 & 120 & 57.4 \\
2 & 110 & 55.4 \\
3 & 100 & 53.0 \\
4 & 90 & 45.8 \\
5 & 80 & 44.6 \\
6 & 70 & 41.4 \\
7 & 60 & 39.4 \\
8 & 50 & n.a. \\
9 & 40 & n.a. \\
10 & 30 & 25.4 \\
11 & 20 & 16.6 \\
12 & 10 & n.a. \\
\hline n.a.=not available. &
\end{tabular}

n.a. $=$ not available.

CALCULATIONS FOR SHOT $($ GRADE $=B)$

Slope $=0.3229$

$\mathrm{V}_{1}=0.94 \mathrm{~km} / \mathrm{s}$

$\mathrm{V}_{2}=3.87 \mathrm{~km} / \mathrm{s}$

Minimum depth to basalt bedrock $=23.34 \mathrm{~m}$ 
TABLE A162. SEISMIC LINE 28, SHOT 19

\begin{tabular}{lll}
\hline Geophone & Distance from Source $(\mathrm{ft})$ & Arrival Time $(\mathrm{ms})$ \\
\hline 1 & 10 & 8.2 \\
2 & 20 & 18.6 \\
3 & 30 & 28.6 \\
4 & 40 & 37.4 \\
5 & 50 & 46.2 \\
6 & 60 & 49.8 \\
7 & 70 & 51.4 \\
8 & 80 & n.a. \\
9 & 90 & n.a. \\
10 & 100 & 47.4 \\
11 & 110 & 48.6 \\
12 & 120 & n.a. \\
\hline
\end{tabular}

CALCULATIONS FOR SHOT $($ GRADE $=C)$

Slope $=0.2600$

$\mathrm{V}_{1}=1.17 \mathrm{~km} / \mathrm{s}$

$\mathrm{V}_{2}=3.87 \mathrm{~km} / \mathrm{s}$

Minimum depth to basalt bedrock $=28.23 \mathrm{~m}$

TABLE A163. SEISMIC LINE 28, SHOT 20

\begin{tabular}{lll}
\hline Geophone & Distance from Source $(\mathrm{ft})$ & Arrival Time $(\mathrm{ms})$ \\
\hline 1 & 120 & 83.4 \\
2 & 110 & 74.2 \\
3 & 100 & 67.0 \\
4 & 90 & 61.4 \\
5 & 80 & 55.4 \\
6 & 70 & 51.4 \\
7 & 60 & 43.8 \\
8 & 50 & n.a. \\
9 & 40 & n.a. \\
10 & 30 & 27.0 \\
11 & 20 & 18.2 \\
12 & 10 & n.a. \\
\hline n.a. $=$ not available. &
\end{tabular}

\section{CALCULATIONS FOR SHOT $(G R A D E=A)$}

Slope $=0.6501$

$\mathrm{V}_{\mathrm{l}}=0.47 \mathrm{~km} / \mathrm{s}$

$\mathrm{V}_{2}=3.87 \mathrm{~km} / \mathrm{s}$

Minimum depth to basalt bedrock $=17.47 \mathrm{~m}$ 
TABLE A164. SEISMIC LINE 28, SHOT 21

\begin{tabular}{lll}
\hline Geophone & Distance from Source $(\mathrm{ft})$ & Arrival Time $(\mathrm{ms})$ \\
\hline $\mathrm{l}$ & 10 & 9.4 \\
2 & 20 & 19.4 \\
3 & 30 & 27.8 \\
4 & 40 & 36.6 \\
5 & 50 & 41.4 \\
6 & 60 & 47.8 \\
7 & 70 & 50.2 \\
8 & 80 & n.a. \\
9 & 90 & n.a. \\
10 & 100 & 51.0 \\
11 & 110 & 53.8 \\
12 & 120 & n.a. \\
\hline n.a. $=$ not available. &
\end{tabular}

CALCULATIONS FOR SHOT (GRADE $=\mathrm{C}$ )

Slope $=0.1576$

$\mathrm{V}_{\mathrm{i}}=1.93 \mathrm{~km} / \mathrm{s}$

$\mathrm{V}_{2}=3.87 \mathrm{~km} / \mathrm{s}$

Minimum depth to basalt bedrock $=50.41 \mathrm{~m}$

TABLE AI65. SEISMIC LINE 28, SHOT 22

\begin{tabular}{lll}
\hline Geophone & Distance from Source $(\mathrm{ft})$ & Arrival Time $(\mathrm{ms})$ \\
\hline 1 & 120 & 53.8 \\
2 & 110 & 51.8 \\
3 & 100 & 50.2 \\
4 & 90 & 48.2 \\
5 & 80 & 46.2 \\
6 & 70 & 45.4 \\
7 & 60 & 45.0 \\
8 & 50 & n.a. \\
9 & 40 & n.a. \\
10 & 30 & 26.2 \\
11 & 20 & 15.8 \\
12 & 10 & n.a. \\
\hline n.a.=not available. &
\end{tabular}

CALCULATIONS FOR SHOT (GRADE $=$ A)

Slope $=0.1543$

$\mathrm{V}_{1}=1.98 \mathrm{~km} / \mathrm{s}$

$\mathrm{V}_{2}=3.87 \mathrm{~km} / \mathrm{s}$

Minimum depth to basalt bedrock $=50.95 \mathrm{~m}$ 
TABLE A 166. SEISMIC LINE 28, SHOT 23

\begin{tabular}{lll}
\hline Geophone & Distance from Source $(\mathrm{ft})$ & Arrival Time $(\mathrm{ms})$ \\
\hline 1 & 10 & 9.8 \\
2 & 20 & 18.6 \\
3 & 30 & 27.8 \\
4 & 40 & 37.4 \\
5 & 50 & 42.6 \\
6 & 60 & 45.4 \\
7 & 70 & 47.4 \\
8 & 80 & n.a. \\
9 & 90 & n.a. \\
10 & 100 & 50.6 \\
11 & 110 & 53.0 \\
12 & 120 & n.a. \\
\hline n.a.=not available. &
\end{tabular}

\section{CALCULATIONS FOR SHOT $(G R A D E=A)$}

Slope $=0.1567$

$\mathrm{V}_{\mathrm{l}}=1.95 \mathrm{~km} / \mathrm{s}$

$\mathrm{V}_{2}=3.87 \mathrm{~km} / \mathrm{s}$

Minimum depth to basalt bedrock $=49.89 \mathrm{~m}$

TABLE A 167. SEISMIC LINE 28, SHOT 24

\begin{tabular}{lll}
\hline Geophone & Distance from Source $(\mathrm{ft})$ & Arrival Time $(\mathrm{ms})$ \\
\hline 1 & 120 & 53.0 \\
2 & 110 & 50.6 \\
3 & 100 & 48.6 \\
4 & 90 & 46.2 \\
5 & 80 & 42.2 \\
6 & 70 & 40.6 \\
7 & 60 & 37.0 \\
8 & 50 & n.a. \\
9 & 40 & n.a. \\
10 & 30 & 27.4 \\
11 & 20 & 17.8 \\
12 & 10 & n.a. \\
\hline n.a.=not available. &
\end{tabular}

CALCULATIONS FOR SHOT $($ GRADE $=\mathrm{A})$

Slope $=0.2844$

$\mathrm{V}_{1}=1.07 \mathrm{~km} / \mathrm{s}$

$\mathrm{V}_{2}=3.87 \mathrm{~km} / \mathrm{s}$

Minimum depth to basalt bedrock $=24.29 \mathrm{~m}$ 
TABLE A 168. SEISMIC LINE 28, SHOT 25

\begin{tabular}{lll}
\hline Geophone & Distance from Source $(\mathrm{ft})$ & Arrival Time $(\mathrm{ms})$ \\
\hline 1 & 10 & 9.0 \\
2 & 20 & 17.4 \\
3 & 30 & 26.2 \\
4 & 40 & 35.8 \\
5 & 50 & n.a. \\
6 & 60 & n.a. \\
7 & 70 & n.a. \\
8 & 80 & n.a. \\
9 & 90 & n.a. \\
10 & 100 & 62.2 \\
11 & 110 & 67.0 \\
12 & 120 & n.a. \\
\hline n.a. $=$ not available. &
\end{tabular}

\section{CALCULATIONS FOR SHOT (GRADE=A)}

Slope $=0.4437$

$\mathrm{V}_{1}=0.69 \mathrm{~km} / \mathrm{s}$

$\mathrm{V}_{2}=3.87 \mathrm{~km} / \mathrm{s}$

Minimum depth to basalt bedrock $=20.37 \mathrm{~m}$

TABLE A 169. SEISMIC LINE 28, SHOT 26

\begin{tabular}{lll}
\hline Geophone & Distance from Source $(\mathrm{ft})$ & Arrival Time $(\mathrm{ms})$ \\
\hline 1 & 120 & 51.4 \\
2 & 110 & 47.8 \\
3 & 100 & 45.4 \\
4 & 90 & 43.0 \\
5 & 80 & 41.8 \\
6 & 70 & 40.2 \\
7 & 60 & 39.8 \\
8 & 50 & n.a. \\
9 & 40 & n.a. \\
10 & 30 & 31.4 \\
11 & 20 & 23.0 \\
12 & 10 & 17.0 \\
\hline n.a. $=$ not available. &
\end{tabular}

CALCULATIONS FOR SHOT (GRADE $=$ A)

Slope $=0.2030$

$\mathrm{V}_{\mathrm{l}}=1.50 \mathrm{~km} / \mathrm{s}$

$\mathrm{V}_{2}=3.87 \mathrm{~km} / \mathrm{s}$

Minimum depth to basalt bedrock $=34.18 \mathrm{~m}$ 
TABLE A170. SEISMIC LINE 28, SHOT 27

\begin{tabular}{lll}
\hline Geophone & Distance from Source $(\mathrm{ft})$ & Arrival Time $(\mathrm{ms})$ \\
\hline 1 & 10 & 7.8 \\
2 & 20 & 18.6 \\
3 & 30 & 28.2 \\
4 & 40 & 34.6 \\
5 & 50 & 38.6 \\
6 & 60 & 43.4 \\
7 & 70 & n.a. \\
8 & 80 & n.a. \\
9 & 90 & n.a. \\
10 & 100 & 49.4 \\
11 & 110 & 52.6 \\
12 & 120 & n.a. \\
\hline
\end{tabular}

n.a. $=$ not available.

CALCULATIONS FOR SHOT (GRADE=B)

Slope $=0.2332$

$\mathrm{V}_{1}=1.31 \mathrm{~km} / \mathrm{s}$

$\mathrm{V}_{2}=3.87 \mathrm{~km} / \mathrm{s}$

Minimum depth to basalt bedrock $=30.51 \mathrm{~m}$

TABLE A 171. SEISMIC LINE 28, SHOT 28

\begin{tabular}{lll}
\hline Geophone & Distance from Source $(\mathrm{ft})$ & Arrival Time $(\mathrm{ms})$ \\
\hline 1 & 120 & 64.6 \\
2 & 110 & 61.0 \\
3 & 100 & 55.8 \\
4 & 90 & 51.4 \\
5 & 80 & 49.0 \\
6 & 70 & 47.4 \\
7 & 60 & 43.4 \\
8 & 50 & n.a. \\
9 & 40 & n.a. \\
10 & 30 & 29.4 \\
11 & 20 & 21.0 \\
12 & 10 & 13.8 \\
\hline
\end{tabular}

n.a. $=$ not available.

\section{CALCULATIONS FOR SHOT (GRADE $=\mathrm{B})$}

Slope $=0.3486$

$\mathrm{V}_{1}=0.87 \mathrm{~km} / \mathrm{s}$

$\mathrm{V}_{2}=3.87 \mathrm{~km} / \mathrm{s}$

Minimum depth to basalt bedrock $=24.76 \mathrm{~m}$ 
TABLE A172. SEISMIC LINE 28, SHOT 29

\begin{tabular}{lll}
\hline Geophone & Distance from Source $(\mathrm{ft})$ & Arrival Time $(\mathrm{ms})$ \\
\hline 1 & 10 & 9.0 \\
2 & 20 & 18.2 \\
3 & 30 & 29.4 \\
4 & 40 & 38.6 \\
5 & 50 & 39.0 \\
6 & 60 & 41.4 \\
7 & 70 & 43.0 \\
8 & 80 & n.a. \\
9 & 90 & n.a. \\
10 & 100 & 45.4 \\
11 & 110 & 46.2 \\
12 & 120 & n.a. \\
\hline n.a. $=$ not available. &
\end{tabular}

CALCULATIONS FOR SHOT $($ GRADE $=\mathbf{A})$

Slope $=0.1121$

$V_{1}=2.72 \mathrm{~km} / \mathrm{s}$

$V_{2}=3.87 \mathrm{~km} / \mathrm{s}$

Minimum depth to basalt bedrock $=71.74 \mathrm{~m}$

TABLE A173. SEISMIC LINE 28, SHOT 30

\begin{tabular}{lll}
\hline Geophone & Distance from Source $(\mathrm{ft})$ & Arrival Time $(\mathrm{ms})$ \\
\hline 1 & 120 & 49.0 \\
2 & 110 & 46.6 \\
3 & 100 & 44.2 \\
4 & 90 & 41.4 \\
5 & 80 & 39.0 \\
6 & 70 & 37.0 \\
7 & 60 & 33.8 \\
8 & 50 & n.a. \\
9 & 40 & n.a. \\
10 & 30 & 25.8 \\
11 & 20 & 17.4 \\
12 & 10 & 7.8 \\
\hline n.a. $=$ not available. &
\end{tabular}

CALCULATIONS FOR SHOT $($ GRADE $=A)$

Slope $=0.2500$

$\mathrm{V}_{1}=1.22 \mathrm{~km} / \mathrm{s}$

$\mathrm{V}_{2}=3.87 \mathrm{~km} / \mathrm{s}$

Minimum depth to basalt bedrock $=25.41 \mathrm{~m}$ 
TABLE A 174. SEISMIC LINE 31, SHOT 1

\begin{tabular}{lll}
\hline Geophone & Distance from Source $(\mathrm{ft})$ & Arrival Time $(\mathrm{ms})$ \\
\hline 1 & 10 & 6.2 \\
2 & 20 & 13.4 \\
3 & 30 & 19.0 \\
4 & 40 & 23.0 \\
5 & 50 & 26.6 \\
6 & 60 & 27.0 \\
7 & 70 & 29.8 \\
8 & 80 & n.a. \\
9 & 90 & n.a. \\
10 & 100 & 39.8 \\
11 & 110 & 42.6 \\
12 & 120 & n.a. \\
\hline n.a.=not available. &
\end{tabular}

\section{CALCULATIONS FOR SHOT (GRADE=B)}

Slope $=0.2872$

$\mathrm{V}_{\mathrm{I}}=1.06 \mathrm{~km} / \mathrm{s}$

$\mathrm{V}_{2}=3.87 \mathrm{~km} / \mathrm{s}$

Minimum depth to basalt bedrock $=18.73 \mathrm{~m}$

TABLE A 175. SEISMIC LINE 31, SHOT 2

\begin{tabular}{lll}
\hline Geophone & Distance from Source $(\mathrm{ft})$ & Arrival Time $(\mathrm{ms})$ \\
\hline 1 & 120 & 48.2 \\
2 & 110 & 50.6 \\
3 & 100 & 51.8 \\
4 & 90 & 50.6 \\
5 & 80 & 46.2 \\
6 & 70 & 41.8 \\
7 & 60 & 38.2 \\
8 & 50 & n.a. \\
9 & 40 & n.a. \\
10 & 30 & 26.6 \\
11 & 20 & 23.0 \\
12 & 10 & n.a. \\
\hline n.a.=not available. &
\end{tabular}

CALCULATIONS FOR SHOT (GRADE=A)

Slope $=0.3912$

$\mathrm{V}_{\mathrm{l}}=0.78 \mathrm{~km} / \mathrm{s}$

$\mathrm{V}_{2}=3.87 \mathrm{~km} / \mathrm{s}$

Minimum depth to basalt bedrock $=17.31 \mathrm{~m}$ 
TABLE A 176. SEISMIC LINE 31, SHOT 3

\begin{tabular}{lll}
\hline Geophone & Distance from Source $(\mathrm{ft})$ & Arrival Time $(\mathrm{ms})$ \\
\hline 1 & 10 & 9.0 \\
2 & 20 & 18.2 \\
3 & 30 & 28.2 \\
4 & 40 & 36.6 \\
5 & 50 & 43.4 \\
6 & 60 & 46.6 \\
7 & 70 & n.a. \\
8 & 80 & n.a. \\
9 & 90 & n.a. \\
10 & 100 & n.a. \\
11 & 110 & n.a. \\
12 & 120 & n.a. \\
\hline n.a.=not available. &
\end{tabular}

CALCULATIONS FOR SHOT $(G R A D E=A)$

Slope $=0.3200$

$\mathrm{V}_{1}=0.95 \mathrm{~km} / \mathrm{s}$

$\mathrm{V}_{2}=3.87 \mathrm{~km} / \mathrm{s}$

Minimum depth to basalt bedrock $=20.58 \mathrm{~m}$

TABLE A177. SEISMIC LINE 31, SHOT 4

\begin{tabular}{lll}
\hline Geophone & Distance from Source (ft) & Arrival Time (ms) \\
\hline 1 & 120 & n.a. \\
2 & 110 & n.a. \\
3 & 100 & 67.8 \\
4 & 90 & n.a. \\
5 & 80 & 57.4 \\
6 & 70 & 51.0 \\
7 & 60 & n.a. \\
8 & 50 & n.a. \\
9 & 40 & n.a. \\
10 & 30 & 27.0 \\
11 & 20 & 17.0 \\
12 & 10 & 12.6 \\
\hline n.a. $=$ not available. &
\end{tabular}

CALCULATIONS FOR SHOT (GRADE $=$ A)

Slope $=0.6586$

$\mathrm{V}_{1}=0.46 \mathrm{~km} / \mathrm{s}$

$\mathrm{V}_{2}=3.87 \mathrm{~km} / \mathrm{s}$

Minimum depth to basalt bedrock $=13.97 \mathrm{~m}$ 
TABLE A178. SEISMIC LINE 31. SHOT 5

\begin{tabular}{lll}
\hline Geophone & Distance from Source $(\mathrm{ft})$ & Arrival Time $(\mathrm{ms})$ \\
\hline 1 & 10 & 7.0 \\
2 & 20 & 16.6 \\
3 & 30 & 25.8 \\
4 & 40 & 33.8 \\
5 & 50 & 43.8 \\
6 & 60 & 52.6 \\
7 & 70 & 62.6 \\
8 & 80 & n.a. \\
9 & 90 & n.a. \\
10 & 100 & 89.4 \\
11 & 110 & 98.6 \\
12 & 120 & n.a. \\
\hline
\end{tabular}

n.a. $=$ not available.

\section{CALCULATIONS FOR SHOT (GRADE $=$ A)}

Slope $=0.9067$

$\mathrm{V}_{1}=0.34 \mathrm{~km} / \mathrm{s}$

$\mathrm{V}_{2}=3.87 \mathrm{~km} / \mathrm{s}$

Minimum depth to basalt bedrock $=15.18 \mathrm{~m}$

TABLE A179. SEISMIC LINE 32, SHOT 1

\begin{tabular}{lll}
\hline Geophone & Distance from Source $(\mathrm{ft})$ & Arrival Time $(\mathrm{ms})$ \\
\hline 1 & 10 & 4.6 \\
2 & 20 & 10.2 \\
3 & 30 & 14.2 \\
4 & 40 & 17.8 \\
5 & 50 & 20.6 \\
6 & 60 & 19.8 \\
7 & 70 & 22.6 \\
8 & 80 & n.a. \\
9 & 90 & n.a. \\
10 & 100 & 22.6 \\
11 & 110 & 23.8 \\
12 & 120 & n.a. \\
\hline n.a. =not available. &
\end{tabular}

CALCULATIONS FOR SHOT $($ GRADE $=C)$

Slope $_{1}=0.4820$, Slope $_{2}=0.0800$

$\mathrm{V}_{\mathrm{I}}=0.63 \mathrm{~km} / \mathrm{s}$

$\mathrm{V}_{2}=3.81 \mathrm{~km} / \mathrm{s}$

Depth to basalt bedrock $=4.81 \mathrm{~m}$ 
TABLE A180. SEISMIC LINE 32, SHOT 2

\begin{tabular}{lll}
\hline Geophone & Distance from Source $(\mathrm{ft})$ & Arrival Time $(\mathrm{ms})$ \\
\hline 1 & 120 & 28.2 \\
2 & 110 & 31.0 \\
3 & 100 & 33.4 \\
4 & 90 & 31.8 \\
5 & 80 & 30.6 \\
6 & 70 & 29.0 \\
7 & 60 & 25.8 \\
8 & 50 & n.a. \\
9 & 40 & n.a. \\
10 & 30 & 20.2 \\
11 & 20 & 13.4 \\
12 & 10 & 6.6 \\
\hline n.a. $=$ not available. &
\end{tabular}

\section{CALCULATIONS FOR SHOT $(G R A D E=A)$}

Slope $=0.1924$

$\mathrm{V}_{1}=1.58 \mathrm{~km} / \mathrm{s}$

$\mathrm{V}_{2}=3.87 \mathrm{~km} / \mathrm{s}$

Minimum depth to basalt bedrock $=22.16 \mathrm{~m}$

TABLE A181. SEISMIC LINE 32, SHOT 3

\begin{tabular}{lll}
\hline Geophone & Distance from Source $(\mathrm{ft})$ & Arrival Time $(\mathrm{ms})$ \\
\hline 1 & 10 & 7.8 \\
2 & 20 & 17.4 \\
3 & 30 & 21.4 \\
4 & 40 & 23.8 \\
5 & 50 & 24.6 \\
6 & 60 & 27.0 \\
7 & 70 & 28.2 \\
8 & 80 & n.a. \\
9 & 90 & n.a. \\
10 & 100 & 31.4 \\
11 & 110 & 33.8 \\
12 & 120 & n.a. \\
\hline n.a. $=$ not available.
\end{tabular}

CALCULATIONS FOR SHOT (GRADE=A)

Slope $=0.1382$

$\mathrm{V}_{1}=2.21 \mathrm{~km} / \mathrm{s}$

$\mathrm{V}_{2}=3.87 \mathrm{~km} / \mathrm{s}$

Minimum depth to basalt bedrock $=33.74 \mathrm{~m}$ 
TABLE A182. SEISMIC LINE 32, SHOT 4

\begin{tabular}{lll}
\hline Geophone & Distance from Source $(\mathrm{ft})$ & Arrival Time $(\mathrm{ms})$ \\
\hline 1 & 120 & 42.2 \\
2 & 110 & 38.2 \\
3 & 100 & 37.0 \\
4 & 90 & 32.6 \\
5 & 80 & 31.4 \\
6 & 70 & 30.2 \\
7 & 60 & 27.8 \\
8 & 50 & n.a. \\
9 & 40 & n.a. \\
10 & 30 & 21.4 \\
11 & 20 & 17.8 \\
12 & 10 & n.a. \\
\hline n.a. $=$ not available. &
\end{tabular}

CALCULATIONS FOR SHOT $($ GRADE $=\mathrm{A})$

Slope $=0.2274$

$\mathrm{V}_{1}=1.34 \mathrm{~km} / \mathrm{s}$

$\mathrm{V}_{2}=3.87 \mathrm{~km} / \mathrm{s}$

Minimum depth to basalt bedrock $=23.40 \mathrm{~m}$

TABLE A183. SEISMIC LINE 32, SHOT 5

\begin{tabular}{lll}
\hline Geophone & Distance from Source $(\mathrm{ft})$ & Arrival Time $(\mathrm{ms})$ \\
\hline 1 & 10 & 6.6 \\
2 & 20 & 17.8 \\
3 & 30 & 22.2 \\
4 & 40 & 27.8 \\
5 & 50 & 31.4 \\
6 & 60 & 41.4 \\
7 & 70 & 46.6 \\
8 & 80 & n.a. \\
9 & 90 & n.a. \\
10 & 100 & 39.0 \\
11 & 110 & 47.8 \\
12 & 120 & n.a. \\
\hline
\end{tabular}

\section{CALCULATIONS FOR SHOT $(G R A D E=A)$}

Slope $=0.4640$

$\mathrm{V}_{1}=0.66 \mathrm{~km} / \mathrm{s}$

$\mathrm{V}_{2}=3.87 \mathrm{~km} / \mathrm{s}$

Minimum depth to basalt bedrock $=9.15 \mathrm{~m}$ 
TABLE A184. SEISMIC LINE 32, SHOT 6

\begin{tabular}{lll}
\hline Geophone & Distance from Source $(\mathrm{ft})$ & Arrival Time $(\mathrm{ms})$ \\
\hline 1 & 120 & 54.6 \\
2 & 110 & 53.8 \\
3 & 100 & 52.6 \\
4 & 90 & 51.4 \\
5 & 80 & 53.0 \\
6 & 70 & 53.4 \\
7 & 60 & 53.4 \\
8 & 50 & n.a. \\
9 & 40 & n.a. \\
10 & 30 & 27.0 \\
11 & 20 & 19.0 \\
12 & 10 & n.a. \\
\hline
\end{tabular}

n.a. $=$ not available.

\section{CALCULATIONS FOR SHOT (GRADE=D)}

Slope $=0.8646$

$\mathrm{V}_{\mathrm{1}}=0.35 \mathrm{~km} / \mathrm{s}$

$\mathrm{V}_{2}=3.87 \mathrm{~km} / \mathrm{s}$

Minimum depth to basalt bedrock $=8.62 \mathrm{~m}$

TABLE A 185. SEISMIC LINE 33, SHOT 1

\begin{tabular}{lll}
\hline Geophone & Distance from Source $(\mathrm{ft})$ & Arrival Time $(\mathrm{ms})$ \\
\hline 1 & 10 & 10.2 \\
2 & 20 & 15.4 \\
3 & 30 & 19.0 \\
4 & 40 & 22.2 \\
5 & 50 & 23.4 \\
6 & 60 & 23.8 \\
7 & 70 & 23.8 \\
8 & 80 & n.a. \\
9 & 90 & n.a. \\
10 & 100 & 27.8 \\
11 & 110 & 30.6 \\
12 & 120 & n.a. \\
\hline
\end{tabular}

n.a.=not available.

CALCULATIONS FOR SHOT $($ GRADE $=A)$

Slope $=0.1214$

$\mathrm{V}_{\mathrm{l}}=2.51 \mathrm{~km} / \mathrm{s}$

$\mathrm{V}_{2}=3.87 \mathrm{~km} / \mathrm{s}$

Minimum depth to basalt bedrock $=36.20 \mathrm{~m}$ 
TABLE AI 86. SEISMIC LINE 33, SHOT 2

\begin{tabular}{lll}
\hline Geophone & Distance from Source $(\mathrm{ft})$ & Arrival Time $(\mathrm{ms})$ \\
\hline 1 & 120 & 37.0 \\
2 & 110 & 36.6 \\
3 & 100 & 36.2 \\
4 & 90 & 35.4 \\
5 & 80 & 33.0 \\
6 & 70 & 29.4 \\
7 & 60 & 27.0 \\
8 & 50 & n.a. \\
9 & 40 & n.a. \\
10 & 30 & 24.2 \\
11 & 20 & 18.6 \\
12 & 10 & n.a. \\
\hline n.a. $=$ not available. &
\end{tabular}

CALCULATIONS FOR SHOT (GRADE=B)

Slope $=0.1494$

$\mathrm{V}_{\mathrm{I}}=2.04 \mathrm{~km} / \mathrm{s}$

$\mathrm{V}_{2}=3.87 \mathrm{~km} / \mathrm{s}$

Minimum depth to basalt bedrock $=33.08 \mathrm{~m}$

TABLE A187. SEISMIC LINE 33, SHOT 3

\begin{tabular}{lll}
\hline Geophone & Distance from Source $(\mathrm{ft})$ & Arrival Time $(\mathrm{ms})$ \\
\hline 1 & 10 & 7.8 \\
2 & 20 & 17.4 \\
3 & 30 & 26.2 \\
4 & 40 & 27.0 \\
5 & 50 & 29.8 \\
6 & 60 & 30.6 \\
7 & 70 & 33.8 \\
8 & 80 & n.a. \\
9 & 90 & n.a. \\
10 & 100 & 41.4 \\
11 & 110 & 43.0 \\
12 & 120 & n.a. \\
\hline
\end{tabular}

n.a. $=$ not available.

CALCULATIONS FOR SHOT (GRADE=B)

Slope $=0.2226$

$V_{1}=1.37 \mathrm{~km} / \mathrm{s}$

$\mathrm{V}_{2}=3.87 \mathrm{~km} / \mathrm{s}$

Minimum depth to basalt bedrock $=25.14 \mathrm{~m}$ 
TABLE A188. SEISMIC LINE 33, SHOT 4

\begin{tabular}{lll}
\hline Geophone & Distance from Source $(\mathrm{ft})$ & Arrival Time $(\mathrm{ms})$ \\
\hline 1 & 120 & 48.2 \\
2 & 110 & 49.0 \\
3 & 100 & 47.8 \\
4 & 90 & 46.6 \\
5 & 80 & 45.0 \\
6 & 70 & 43.8 \\
7 & 60 & 43.8 \\
8 & 50 & n.a. \\
9 & 40 & n.a. \\
10 & 30 & 27.0 \\
11 & 20 & 17.0 \\
12 & 10 & 7.8 \\
\hline n.a. $=$ not available. &
\end{tabular}

CALCULATIONS FOR SHOT (GRADE $=$ A)

Slope $=0.1320$

$\mathrm{V}_{\mathrm{l}}=2.31 \mathrm{~km} / \mathrm{s}$

$\mathrm{V}_{2}=3.87 \mathrm{~km} / \mathrm{s}$

Minimum depth to basalt bedrock $=58.05 \mathrm{~m}$

TABLE A1 89. SEISMIC LINE 33, SHOT 5

\begin{tabular}{lll}
\hline Geophone & Distance from Source $(\mathrm{ft})$ & Arrival Time $(\mathrm{ms})$ \\
\hline 1 & 10 & 9.8 \\
2 & 20 & 18.2 \\
3 & 30 & 27.4 \\
4 & 40 & 36.6 \\
5 & 50 & 45.0 \\
6 & 60 & 50.2 \\
7 & 70 & 52.2 \\
8 & 80 & n.a. \\
9 & 90 & n.a. \\
10 & 100 & 55.4 \\
11 & 110 & 57.4 \\
12 & 120 & n.a. \\
\hline n.a.=not available. &
\end{tabular}

CALCULATIONS FOR SHOT (GRADE $=B$ )

Slope $=0.1341$

$\mathrm{V}_{1}=2.27 \mathrm{~km} / \mathrm{s}$

$\mathrm{V}_{2}=3.87 \mathrm{~km} / \mathrm{s}$

Minimum depth to basalt bedrock $=68.46 \mathrm{~m}$ 
TABLE A190. SEISMIC LINE 33, SHOT 6

\begin{tabular}{lll}
\hline Geophone & Distance from Source $(\mathrm{ft})$ & Arrival Time $(\mathrm{ms})$ \\
\hline 1 & 120 & 85.0 \\
2 & 110 & 82.2 \\
3 & 100 & 77.4 \\
4 & 90 & 73.0 \\
5 & 80 & 69.4 \\
6 & 70 & 67.0 \\
7 & 60 & 54.6 \\
8 & 50 & n.a. \\
9 & 40 & n.a. \\
10 & 30 & 27.8 \\
11 & 20 & 17.8 \\
12 & 10 & n.a. \\
\hline n.a. $=$ not available. &
\end{tabular}

CALCULATIONS FOR SHOT $($ GRADE $=\mathrm{A})$

Slope $=0.3794$

$\mathrm{V}_{\mathrm{I}}=0.80 \mathrm{~km} / \mathrm{s}$

$\mathrm{V}_{2}=3.87 \mathrm{~km} / \mathrm{s}$

Minimum depth to basalt bedrock $=31.03 \mathrm{~m}$

TABLE A191. SEISMIC LINE 33, SHOT 7

\begin{tabular}{lll}
\hline Geophone & Distance from Source $(\mathrm{ft})$ & Arrival Time $(\mathrm{ms})$ \\
\hline 1 & 10 & 8.2 \\
2 & 20 & 17.8 \\
3 & 30 & 27.0 \\
4 & 40 & 35.8 \\
5 & 50 & 45.4 \\
6 & 60 & 54.2 \\
7 & 70 & 63.0 \\
8 & 80 & n.a. \\
9 & 90 & n.a. \\
10 & 100 & 92.2 \\
11 & 110 & 98.6 \\
12 & 120 & n.a. \\
\hline
\end{tabular}

CALCULATIONS FOR SHOT (GRADE $=$ A)

Slope $=0.9107$

$\mathrm{V}_{\mathrm{l}}=0.33 \mathrm{~km} / \mathrm{s}$

$\mathrm{V}_{2}=3.87 \mathrm{~km} / \mathrm{s}$

Minimum depth to basalt bedrock $=15.11 \mathrm{~m}$ 
TABLE A192. SEISMIC LINE 33, SHOT 8

\begin{tabular}{lll}
\hline Geophone & Distance from Source $(\mathrm{ft})$ & Arrival Time $(\mathrm{ms})$ \\
\hline 1 & 120 & 109.4 \\
2 & 110 & 99.4 \\
3 & 100 & 92.2 \\
4 & 90 & 86.6 \\
5 & 80 & 70.6 \\
6 & 70 & 63.4 \\
7 & 60 & 54.6 \\
8 & 50 & n.a. \\
9 & 40 & n.a. \\
10 & 30 & 27.4 \\
11 & 20 & 17.4 \\
12 & 10 & 9.4 \\
\hline n.a. $=$ not available. &
\end{tabular}

CALCULATIONS FOR SHOT (GRADE $=$ A)

Slope $=0.9172$

$\mathrm{V}_{\mathrm{I}}=0.33 \mathrm{~km} / \mathrm{s}$

$\mathrm{V}_{2}=3.87 \mathrm{~km} / \mathrm{s}$

Minimum depth to basalt bedrock $=16.67 \mathrm{~m}$

TABLE A 193. SEISMIC LINE 35, SHOT I

\begin{tabular}{lll}
\hline Geophone & Distance from Source $(\mathrm{ft})$ & Arrival Time $(\mathrm{ms})$ \\
\hline 1 & 0 & 0.0 \\
2 & 30 & 26.2 \\
3 & 60 & 53.0 \\
4 & 90 & 79.4 \\
5 & 120 & 82.2 \\
6 & 150 & 84.6 \\
7 & 180 & 90.6 \\
8 & 210 & n.a. \\
9 & 240 & n.a. \\
10 & 270 & n.a. \\
11 & 300 & n.a. \\
12 & 330 & n.a. \\
\hline
\end{tabular}

n.a. $=$ not available.

CALCULATIONS FOR SHOT (GRADE $=$ B)

Slope $_{1}=0.8333$, Slope $_{2}=0.0867$

$\mathrm{V}_{1}=0.37 \mathrm{~km} / \mathrm{s}$

$\mathrm{V}_{2}=3.52 \mathrm{~km} / \mathrm{s}$

Depth to basalt bedrock $=13.18 \mathrm{~m}$ 
TABLE A194. SEISMIC LINE 35, SHOT 2

\begin{tabular}{lll}
\hline Geophone & Distance from Source $(\mathrm{ft})$ & Arrival Time $(\mathrm{ms})$ \\
\hline $\mathrm{l}$ & 330 & n.a. \\
2 & 300 & n.a. \\
3 & 270 & 125.4 \\
4 & 240 & n.a. \\
5 & 210 & n.a. \\
6 & 180 & 117.0 \\
7 & 150 & 109.4 \\
8 & 120 & n.a. \\
9 & 90 & n.a. \\
10 & 60 & 55.0 \\
11 & 30 & n.a. \\
12 & 0 & 0.0 \\
\hline n.a.=not available. &
\end{tabular}

\section{CALCULATIONS FOR SHOT $($ GRADE $=C)$}

Slope $=0.0933$

$\mathrm{V}_{1}=3.27 \mathrm{~km} / \mathrm{s}$

$\mathrm{V}_{2}=3.87 \mathrm{~km} / \mathrm{s}$

Minimum depth to basalt bedrock $=317.56 \mathrm{~m}$

TABLE A195. SEISMIC LINE 35, SHOT 3

\begin{tabular}{lll}
\hline Geophone & Distance from Source $(\mathrm{ft})$ & Arrival Time $(\mathrm{ms})$ \\
\hline 1 & 210 & n.a. \\
2 & 180 & n.a. \\
3 & 150 & 11.0 \\
4 & 120 & 107.4 \\
5 & 90 & 80.6 \\
6 & 60 & 63.4 \\
7 & 30 & 45.0 \\
8 & 0 & 0.0 \\
9 & 30 & n.a. \\
10 & 60 & n.a. \\
11 & 90 & n.a. \\
12 & 120 & n.a. \\
\hline n.a.=not available. &
\end{tabular}

CALCULATIONS FOR SHOT $($ GRADE $=\mathrm{C})$

Slope $=0.1200$

$\mathrm{V}_{\mathrm{1}}=2.54 \mathrm{~km} / \mathrm{s}$

$\mathrm{V}_{2}=3.87 \mathrm{~km} / \mathrm{s}$

Minimum depth to basalt bedrock $=167.03 \mathrm{~m}$ 